Florida International University FIU Digital Commons

2012

\title{
Geographic Provenancing of Unprocessed Cotton Using Elemental Analysis and Stable Isotope Ratios
}

Emily R. Schenk

Florida International University, esche003@fiu.edu

DOI: $10.25148 /$ etd.FI12113008

Follow this and additional works at: https://digitalcommons.fiu.edu/etd

\section{Recommended Citation}

Schenk, Emily R., "Geographic Provenancing of Unprocessed Cotton Using Elemental Analysis and Stable Isotope Ratios" (2012). FIU Electronic Theses and Dissertations. 803.

https://digitalcommons.fiu.edu/etd/803 


\section{FLORIDA INTERNATIONAL UNIVERSITY}

Miami, FL

GEOGRAPHIC PROVENANCING OF UNPROCESSED COTTON USING ELEMENTAL ANALYSIS AND STABLE ISOTOPE RATIOS

A dissertation submitted in partial fulfillment of the

Requirements for the degree of

DOCTOR OF PHILOSOPHY

in

CHEMISTRY

by

Emily Rose Schenk

2012 


\section{To: Dean Kenneth G. Furton}

College of Arts and Sciences

This dissertation, written by Emily Rose Schenk, and entitled Geographic Provenancing of Unprocessed Cotton Using Elemental Analysis and Stable Isotope Ratios, having been approved in respect to style and intellectual content, is referred to you for judgment.

We have read this dissertation and recommend that it be approved.

$\begin{array}{r}\text { Kenneth Furton } \\ \hline \text { Jaroslava Miksovska } \\ \hline \text { Watson Lees } \\ \hline \text { Jose Almirall, Major Professor }\end{array}$

Date of Defense: November 7, 2012

The dissertation of Emily Rose Schenk is approved.

Dean Kenneth G. Furton

College of Arts and Sciences

Dean Lakshmi N. Reddi University Graduate School

Florida International University, 2012 
(C) Copyright 2012 by Emily Rose Schenk

All rights reserved. 


\section{DEDICATION}

I would like to dedicate my dissertation to my family and especially my parents, Bill and Donna. My parents have always whole-heartedly supported me in whatever path in life has made me happy and that included pursuing a doctorate in chemistry. Since I was little, they took every opportunity to enrich my mind with weekend trips to museums, galleries and fossil hunting trips. They exposed me to science and chemistry and for that I am grateful. They gave me the world and so much more.

I would also like to dedicate this to those whom have provided encouragement, a listening ear and a shoulder to lean on, now and always.

Together you all gave light to my dreams.

"Go confidently in the direction of your dreams. Live the life you have imagined." -Henry David Thoreau 


\section{ACKNOWLEDGMENTS}

I have thoroughly enjoyed my time in the Chemistry graduate program at Florida International University. I have had the opportunity to work along-side many knowledgeable, talented individuals whom deserve the acknowledgements given here and so much more.

I would like to first and foremost acknowledge and thank Dr. Almirall for providing me with the opportunity to work in his research lab. I greatly appreciate the chance to attend several scientific meetings and participate in workshops. His guidance and knowledge has allowed me to grow as a scientist. I would also like to acknowledge my dissertation committee for their time and advice through the years. Thank you, Dr. Furton, Dr. Miksovska, Dr. Hickey-Vargas and Dr. Lees, for your support.

I am also thankful for the financial support provided by UGS and GSFC to fund my travels to scientific meetings, and especially UGS for awarding me the DEA fellowship.

This work would not be possible without the kindness of several individuals and companies who willingly and graciously took their time to provide me with samples for this research. In no particular order I would like to acknowledge and thank Ed Barnes and Dr. Don Jones at Cotton Incorporated, the farmers and researchers involved with the Regional Breeders Testing Network (RBTN), Elena Bonny at Softlooms, Mike Beeker at Frontier Spinning Mills, Inc., Keith Maloney at the Dumas, AR Cotton Classing Office, Roy Lockett at Hamrick Mills, Gary Gamble at the USDA and Janice Gerde at US Customs. These individuals took time out of their day(s) to organize and document cotton samples for this research. I cannot thank them enough. The stable isotope ratio analysis was conducted at the Mylnefield Research Services (MRS) facility in Dundee, Scotland under the advisement of Professor Wolfram Meier-Augenstein. Special thanks goes out to Professor Meier-Augenstein for taking the time to explain procedures and assist in data analysis and to Helen Kemp for conducting the analysis. 
My research group and friends in the graduate program have been here day after day providing support when research was not going well and when it was successful. I would like to thank the “A-team" both present and past - Monica Joshi-Kumar, Howard Holness, Mimy Young, Wen Fan, Anamary Tarifa, Melissa Zwilling, Seongshin Gwak (Shin), and especially the "laaassersss" - Erica Cahoon, Sarah Jantzi, Maria Perez and Tatiana Trejos. I would also like to thank the undergraduate students who have contributed and helped along the way - Kia Rigsby, Nick Weis and Josh Silverman. 
ABSTRACT OF THE DISSERTATION

GEOGRAPHIC PROVENANCING OF UNPROCESSED COTTON USING ELEMENTAL

ANALYSIS AND STABLE ISOTOPE RATIOS

by

Emily Rose Schenk

Florida International University, 2012

Miami, FL

Professor Jose Almirall, Major Professor

Cotton is the most abundant natural fiber in the world. Many countries are involved in the growing, importation, exportation and production of this commodity. Paper documentation claiming geographic origin is the current method employed at U.S. ports for identifying cotton sources and enforcing tariffs. Because customs documentation can be easily falsified, it is necessary to develop a robust method for authenticating or refuting the source of the cotton commodities. This work presents, for the first time, a comprehensive approach to the chemical characterization of unprocessed cotton in order to provide an independent tool to establish geographic origin. Elemental and stable isotope ratio analysis of unprocessed cotton provides a means to increase the ability to distinguish cotton in addition to any physical and morphological examinations that could be, and are currently performed. Elemental analysis has been conducted using LA-ICP-MS, LA-ICP-OES and LIBS in order to offer a direct comparison of the analytical performance of each technique and determine the utility of each technique for this purpose.

Multivariate predictive modeling approaches are used to determine the potential of elemental and stable isotopic information to aide in the geographic provenancing of unprocessed cotton of both domestic and foreign origin. These approaches assess the stability of the profiles to temporal and spatial variation to determine the feasibility of this application. 
This dissertation also evaluates plasma conditions and ablation processes so as to improve the quality of analytical measurements made using atomic emission spectroscopy techniques. These interactions, in LIBS particularly, are assessed to determine any potential simplification of the instrumental design and method development phases. This is accomplished through the analysis of several matrices representing different physical substrates to determine the potential of adopting universal LIBS parameters for $532 \mathrm{~nm}$ and $1064 \mathrm{~nm}$ LIBS for some important operating parameters. A novel approach to evaluate both ablation processes and plasma conditions using a single measurement was developed and utilized to determine the "useful ablation efficiency" for different materials. The work presented here demonstrates the potential for an a priori prediction of some probable laser parameters important in analytical LIBS measurement. 


\section{TABLE OF CONTENTS}

\section{CHAPTER}

\section{INTRODUCTION}

1.1 Current State of the Problem.......................................................

1.2 Research Motivation and Proposed Solutions.........................................

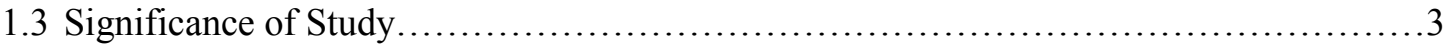

\section{PART 1: STUDIES OF PLASMA INTERACTIONS IN ATOMIC EMISSION SPECTROSCOPY}

2 STRATEGY FOR STANDARDIZATION OF ANALYTICAL LIBS METHODS THROUGH THE DEVELOPMENT OF UNIVERSAL PARAMETERS

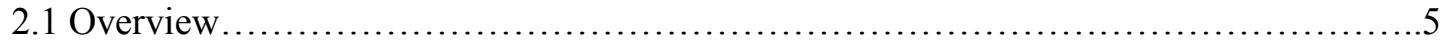

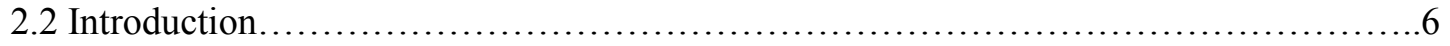

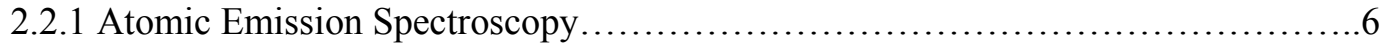

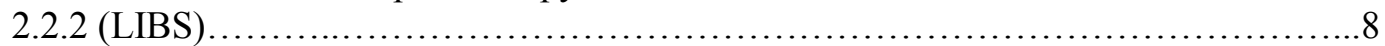

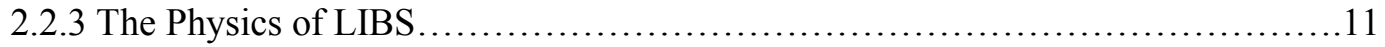

2.2.4 Introduction to Analytical LIBS and the Need for Standardization in Operating

Conditions................................................................

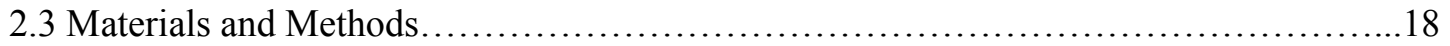

2.3.1 Description of Samples and Sample Preparation................................ 18

2.3.1.1 Cotton Matrix....................................................... 18

2.3.1.2 Glass Matrix............................................................. 18

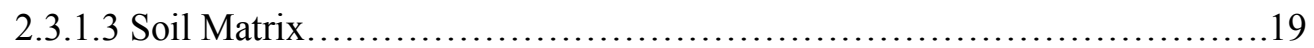

2.3.2 LIBS Experimental Design.................................................. 19

2.3.2.1 UV (266nm) LIBS In-house Setup...................................19

2.3.2.2 Vis $(532 \mathrm{~nm})$ LIBS In-house Setup........................................20

2.3.2.3 IR (1064nm) LIBS In-house Setup.................................20

2.3.2.4 RT-100 HP Commercial LIBS Setup...................................21

2.3.4 Determination of Laser Beam Characteristics..................................21

2.3.5 Determination of Mass Removal and Crater Morphology.........................22

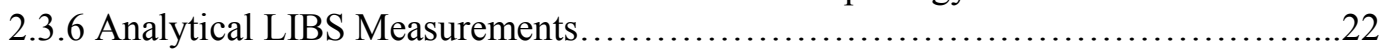

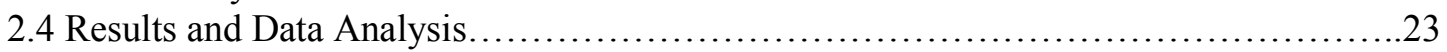

2.4.1 Laser Beam Characteristics...............................................23

2.4.2 Analytical Performance of Unprocessed Cotton Using Different Laser Power

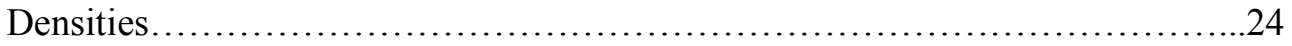

2.4.3 Standardization of Laser Power Density for Different Matrices....................28

2.4.4 Cotton Crater Development to Address Standardization in Aspect Ratio...........29

2.4.5 Novel Approach to Relating Plasma and Ablation Processes.............................33

2.5 Discussion and Conclusions.................................................... 35

3 AN APPROACH FOR MEDIATING MATRIX EFFECTS IN ICP-OES

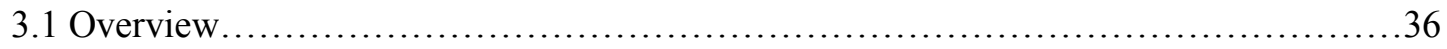

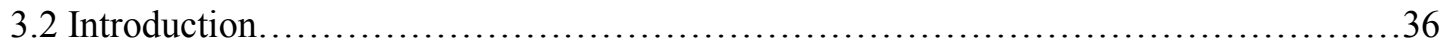

3.2.1 Plasma interactions and dynamics in ICP-OES ............................... 36

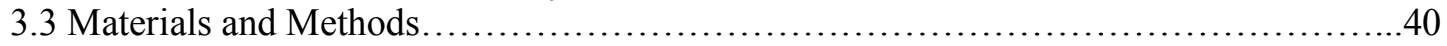

3.3.1 Description of Samples and Sample Preparation..............................40 
3.3.1.1 Matrix Solution Preparation........................................40

3.3.1.2 Analyte Solution Preparation........................................42

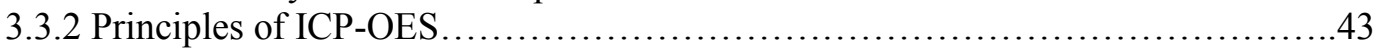

3.3.3 ICP-OES Experimental Design......................................... 48

3.3.3.1 ICP-OES Setup and Instrumentation.................................48

3.3.4 Criteria for Evaluation of Studies.......................................... 50

3.4 Results and Data Analysis...................................................... 51

3.4.1 Influence of EIEs and NEIEs on Ionic and Atomic Line Response.................51

3.4.2 Zinc as a Matrix Buffer...................................................... 56

3.4.3 Implications of Buffering on Analytical Performance...........................58

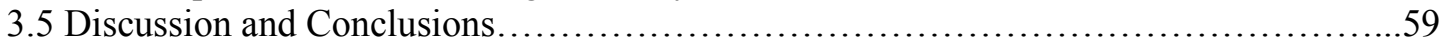

\section{INFLUENCE OF GAS COMPOSITION ON LA-ICP-OES PERFORMANCE}

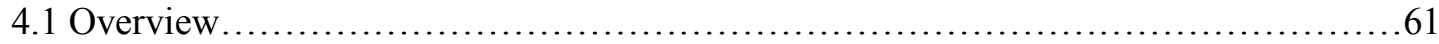

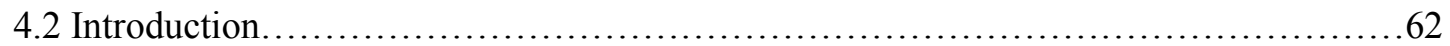

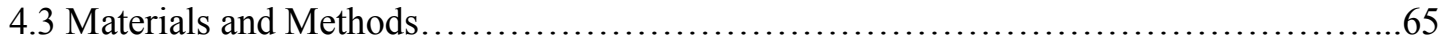

4.3.1 Description of Samples and Sample Preparation...............................65

4.3.1.1 Cotton Matrix........................................................65

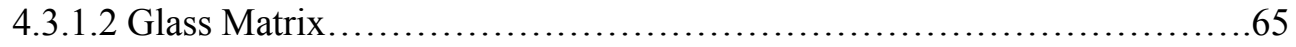

4.3.2 Principles of Laser Ablation for ICP techniques...............................66

4.3.3 LA-ICP-OES Experimental Design..........................................68

4.3.3.1 Laser Ablation Units...............................................68

4.3.3.2 ICP-OES Setup and Instrumentation.................................69

4.3.3.3 Manipulation of External Gas Introduction.............................71

4.3.4 Indirect Determination of Particle and Aggregate Size of Ablated Material...........71

4.3.5 Daily Performance and Quality Control of LA-ICP-OES Setup....................73

4.3.6 Generation of a Transient Signal.............................................74

4.3.7 Criteria for Evaluation of Studies........................................... 74

4.4 Results and Data Analysis....................................................... 76

4.4.1 Comparison of Argon or Helium as Entire Central Channel Gas Composition......76

4.4.2 Assessment of He-Ar and Ar-He Mixtures for the Central Channel Gas

Composition...........................................................79

4.4.3 Influence of Central Channel Gas Composition of Particle Size Distribution.......85

4.4.4 Effect of Transport Tubing Length............................................ 88

4.4.5 Influence of Central Channel Gas on Analytical Performance.....................90

4.5 Discussion and Conclusions................................................. 91

\section{PART 2: FORENSIC APPLICATIONS}

\section{FORENSIC GLASS ANALYSIS BY LA-ICP-OES}

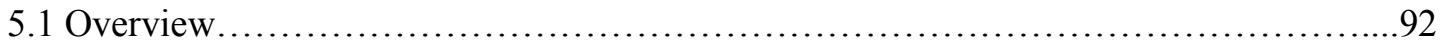

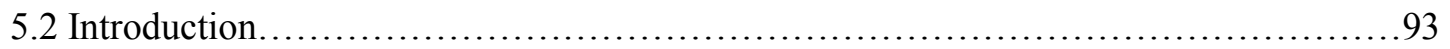

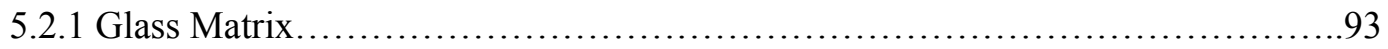

5.2.2 Forensic Analysis of Glass............................................... 97

5.3 Materials and Methods....................................................... 102

5.3.1 Description of Glass Standards and Samples............................... 102

5.3.1.1 Use of Glass SRMs for Calibration and Quantification...................102

5.3.1.2 Description of the Sample Set...................................... 104

5.3.1.3 Preparation and Sampling Scheme of the Glass Samples.................105 


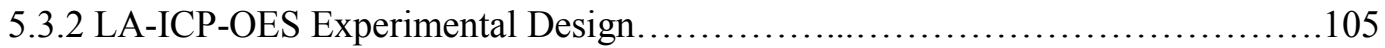

5.3.2.1 LA-ICP-OES Setup and Instrumentation.............................. 105

5.3.2.2 Element Menu................................................... 106

5.4 Results and Data Analysis................................................... 108

5.4.1 Transient Signal Integration and Corresponding Concentration Evaluation........108

5.4.2 Calculating LODs and LOQs.............................................. 109

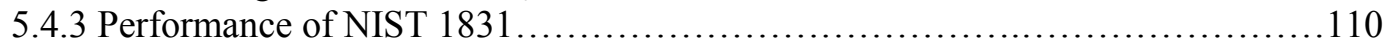

5.4.4 Analytical Performance of the LA-ICP-OES Method...........................112

5.4.5 Statistical Analysis of Vehicle Glass sample Set................................112

5.4.5.1 Considerations of Data Interpretation............................... 112

5.4.5.1.1 Discrimination Techniques...............................112

5.4.5.1.2 Types of error and rules for data interpretation.................115

5.4.5.2 Interpretation of the sample set..................................... 116

5.5 Discussion and Conclusions.................................................. 120

6 APPLICATION OF LA-ICP-OES AND LIBS FOR THE ELEMENTAL ANALYSIS OF UNPROCESSED COTTON

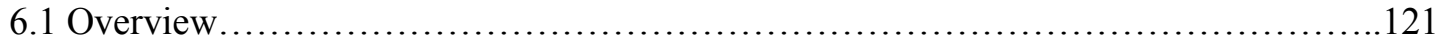

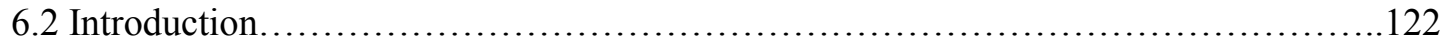

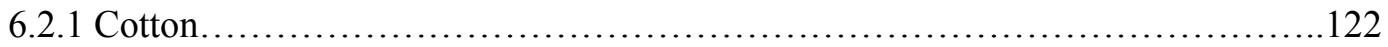

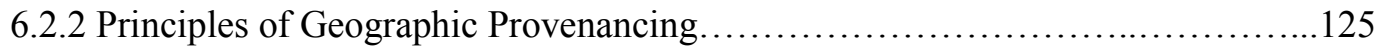

6.3 Materials and Methods.......................................................... 126

6.3.1 Description of Samples and Sampling Scheme................................ 126

6.3.1.1 Cotton Sampling Strategy........................................... 126

6.3.1.2 Cotton Pellet Preparation............................................ 128

6.3.1.3 Cotton SRM...................................................... 130

6.3.1.4 Development of an In-house Unprocessed Cotton Standard..............132

6.3.1.5 Description of Test Sample Set.......................................135

6.3.2 Comparison of LA-ICP-OES and LIBS ......................................... 137

6.3.3 LA-ICP-OES Experimental Design....................................... 137

6.3.3.1 LA-ICP-OES Setup and Instrumentation..............................137

6.3.3.2 Element Menu...................................................... 138

6.3.4 LIBS Experimental Design........................................... 138

6.3.4.1 LIBS Setup and instrumentation......................................138

6.3.4.2 Role of Shadowgraphy in LIBS Optimization...........................140

6.3.4.3 Element Menu...................................................147

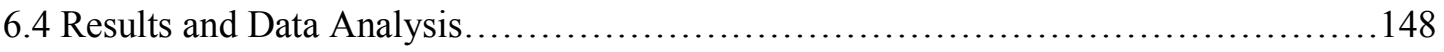

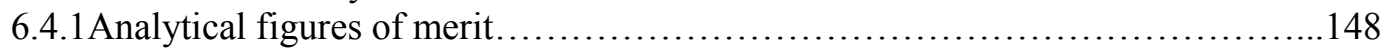

6.4.1.1 LIBS Analytical Figures of Merit...................................148

6.4.1.2 LA-ICP-OES Analytical Figures of Merit...............................150

6.4.2 Comparison of Analytical Ability......................................... 152

6.4.3 Performance of Sample Set............................................... 154

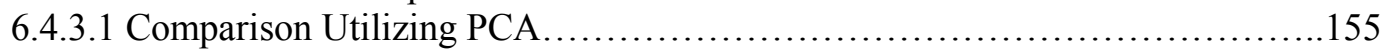

6.5 Discussion and Conclusions................................................ 159

7 COMPARISON OF ATOMIC EMISSION TECHNIQUES TO LA-ICP-MS

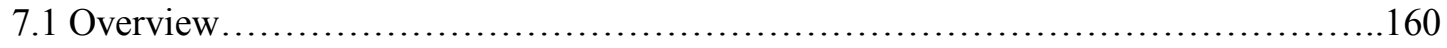

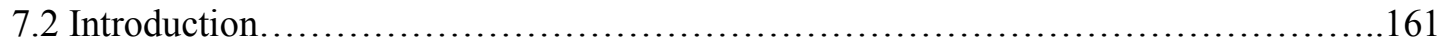

7.2.1 Elemental Analysis Techniques......................................... 161 
7.3 Materials and Methods........................................................... 162

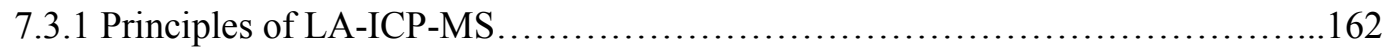

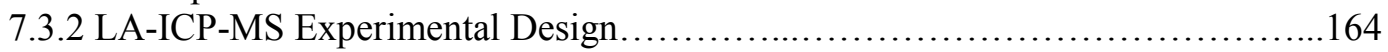

7.3.2.1 LA-ICP-MS Setup and Instrumentation............................... 164

7.3.2.2 Element Menu..................................................... 166

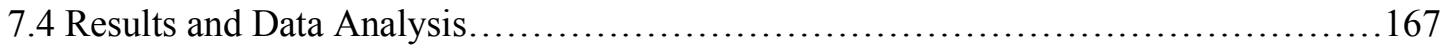

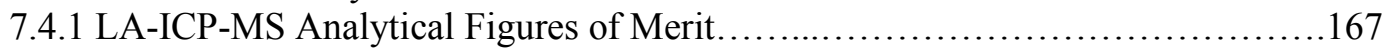

7.4.2 Performance Utilizing a Sample Set......................................170

7.4.3.1 Comparison to LA-ICP-OES and LIBS .............................171

7.5 Discussion and Conclusions................................................ 173

8 DETERMINATION OF THE STABLE ISOTOPES IN UNPROCESSED COTTON

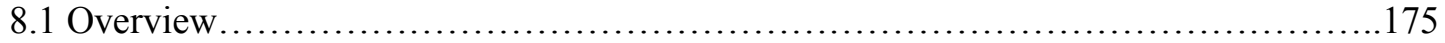

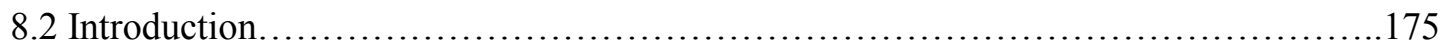

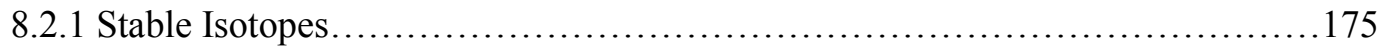

8.2.2 Use of Stable Isotopes for Geographic Provenancing............................177

8.3 Materials and Methods........................................................... 181

8.3.1 Description of Samples and Sample Preparation............................... 181

8.3.1.1 Description of Samples........................................... 181

8.3.1.1.1 Domestic Unprocessed Cotton...........................181

8.3.1.1.2 Foreign Unprocessed Cotton............................182

8.3.1.2 Sample Preparation............................................. 183

8.3.1.2.1 Equilibration of Samples................................ 183

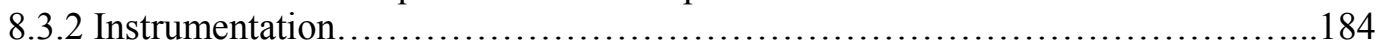

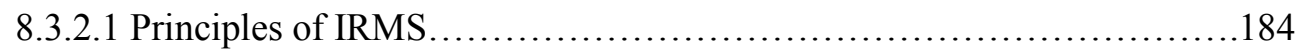

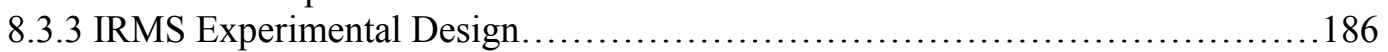

8.3.3.1 IRMS Setup and Instrumentation................................... 186

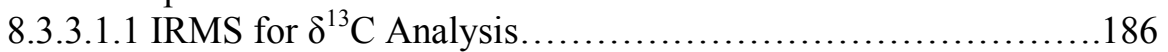

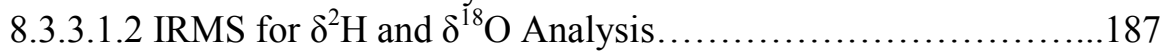

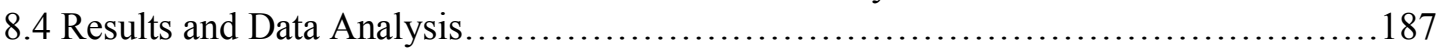

8.4.1 Analytical Performance.................................................. 187

8.4.2 IRMS Analysis of Domestic and Foreign Unprocessed Cotton Samples............190

8.4.2.1 Univariate Comparison of $\delta^{13} \mathrm{C}, \delta^{2} \mathrm{H}$ and $\delta^{18} \mathrm{O}$ Results....................190

8.4.2.2 Statistical Evaluation.................................................196

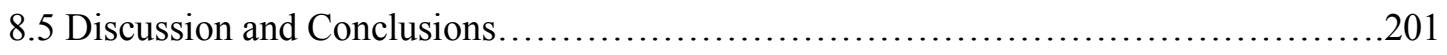

9 EVALUATION OF MULTIVARIATE STATISTICAL APPROACHES FOR

GEOGRAPHIC PROVENANCING OF UNPROCESSED COTTON

9.1

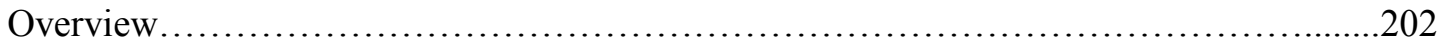

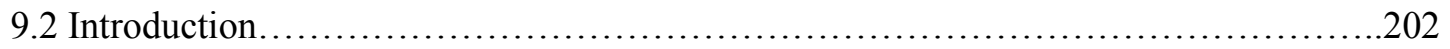

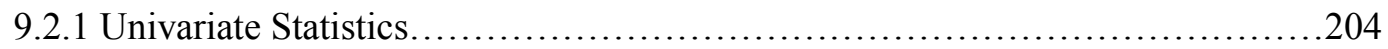

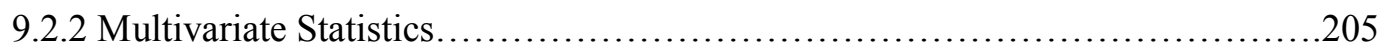

9.2.2.1 Visual Exploration...............................................206

9.2.2.2 Predictive Modeling Techniques...................................207

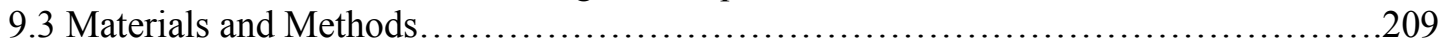

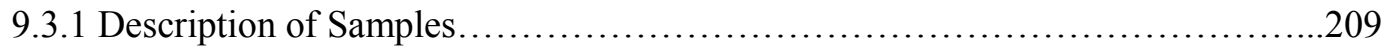

9.3.1.1 Domestic Sample Sets..........................................209

9.3.1.1.1 Influence of Crop Harvest Method Sample Set................209 
9.3.1.1.2 Influence on Sample Storage Techniques Sample Set..........210

9.3.1.1.3 Intra and Inter-variation in Cotton Bales.......................210

9.3.1.1.4 Variation within a Field Sample Set........................211

9.3.1.1.5 RBTN 2009 Harvest Sample Set.............................211

9.3.1.1.6 Frontier Spinning Mills 2010 Sample Set....................212

9.3.1.1.7 Arkansas Cotton Classing Office 2011 Sample Set.............212

9.3.1.2 Foreign Sample Set..............................................213

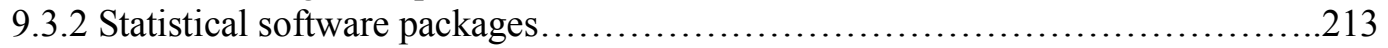

9.3.3 Parameter Selection for Modeling the Data .................................. 214

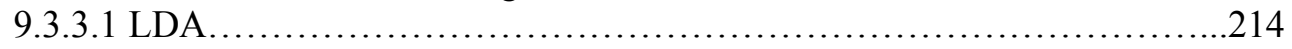

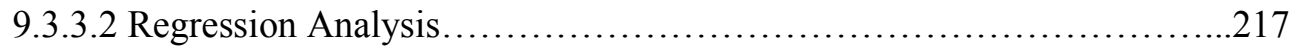

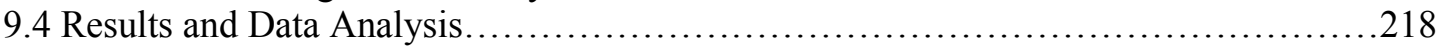

9.4.1 Addressing Heterogeneity of the Samples.....................................218

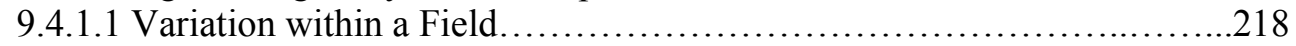

9.4.1.2 Intra and Inter-variation in Cotton Bales................................219

9.4.1.3 Influence of Crop Harvest Method....................................220

9.4.1.4 Influence of Cotton Variety on Elemental Profile........................222

9.4.1.5 Influence of Sample Storage Technique................................222

9.4.2 Geographic Provenancing of Unprocessed Cotton Samples.....................223

9.4.2.1 LDA: Classification of Domestic Samples...........................225

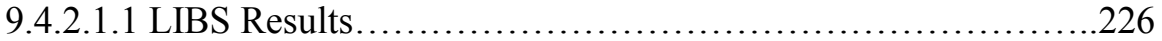

9.4.2.1.2 LA-ICP-OES Results...................................228

9.4.2.1.3 LA-ICP-MS Results....................................229

9.4.2.2 Addressing the Elemental Profile of the U.S. Using Geographic Maps...231

9.4.2.3 LDA: Classification of Foreign and Domestic Samples....................232

9.4.2.3.1 LIBS Results..............................................232

9.4.2.3.2 LA-ICP-OES Results....................................233

9.4.2.3.3 LA-ICP-MS Results.......................................234

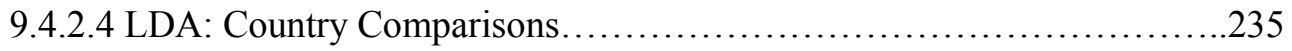

9.4.2.4.1 LA-ICP-OES Results.................................236

9.4.3 Establishing Geographic Trends Using Elemental and Stable Isotope

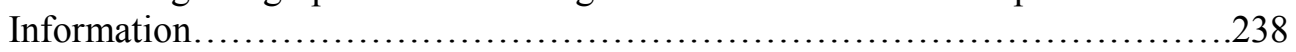

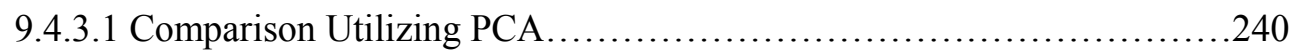

9.4.4 Regression Analysis..................................................... 242

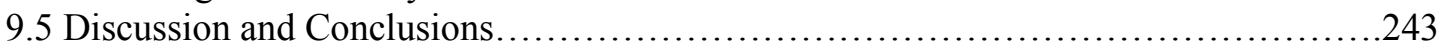

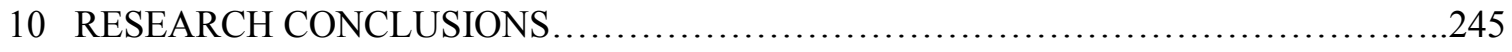

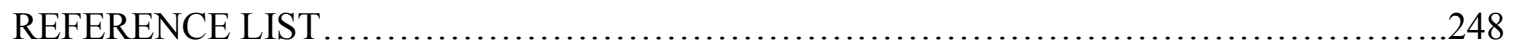

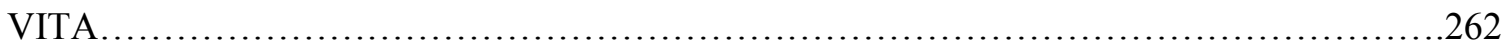




\section{LIST OF TABLES}

TABLE

PAGE

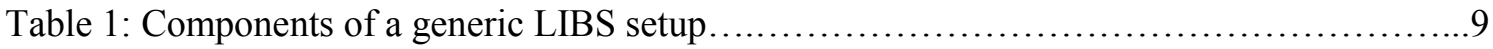

Table 2: Important parameters in LIBS optimization and method development...................15

Table 3: Cotton and glass LIBS parameters for $266 \mathrm{~nm}, 532 \mathrm{~nm}$ and $1064 \mathrm{~nm}$ LIBS analysis.....23

Table 4: $532 \mathrm{~nm}$ and $1064 \mathrm{~nm}$ laser beam properties.....................................24

Table 5: Analytical performance of $532 \mathrm{~nm}$ and $1064 \mathrm{~nm}$ LIBS for cotton analysis...............25

Table 6: Power densities for different matrices using optimal LIBS parameters .................28

Table 7: Calculated crater aspect ratios for different cotton LIBS analyses.......................31

Table 8: Ablation efficiencies determined by $532 \mathrm{~nm}$ and $1064 \mathrm{~nm}$ LIBS analysis of cotton......34

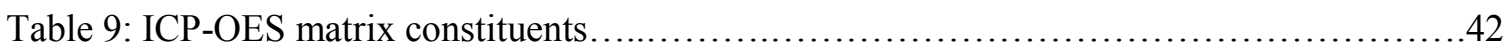

Table 10: Important variable information for electron density calculations...................52

Table 11: Single analyte effects in the presence of $\mathrm{Zn}$ and other matrix effects (in \%

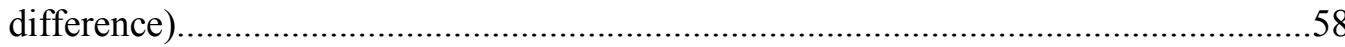

Table 12: Analytical performance in the presence of matrix effects and buffers................60

Table 13: Robust plasma conditions for glass and cotton................................. 79

Table 14: Comparison of SRM NIST 1831 ablation using $213 \mathrm{~nm}$ and $266 \mathrm{~nm}$ wavelengths lasers

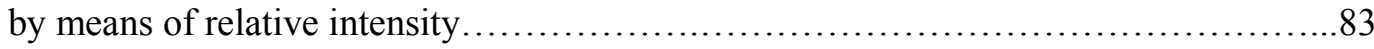

Table 15: Analytical performance for different gas configurations in LA-ICP-OES..............90

Table 16: Common glass constituents................................................97

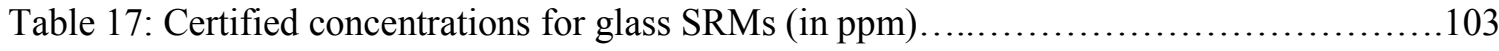

Table 18: Vehicle sample set information for LA-ICP-OES analysis..........................104

Table 19: Element emission lines used in LA-ICP-OES glass analysis.....................108 


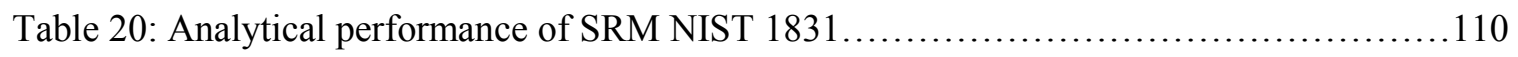

Table 21: LODs and LOQs for the LA-ICP-OES analysis of glass..........................112

Table 22: Resulting indistinguishable pairs from the vehicle glass analysis using ANOVA with

Tukey's and the t-test at the $95 \%$ C.I. ...................................... 117

Table 23: Indistinguishable pairs obtained when broader match criteria is used to interpret LA-

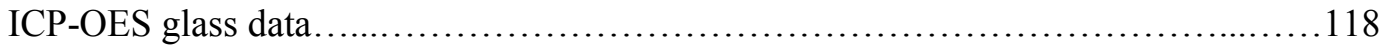

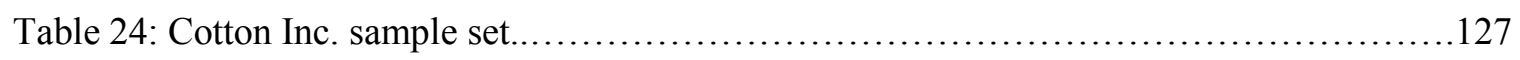

Table 25: Effect of mill time on particle size distribution of unprocessed cotton................130

Table 26: IAEA V-9 cotton cellulose SRM composition...................................132

Table 27: Baseline concentration of some analytes in NC2005 reported with different

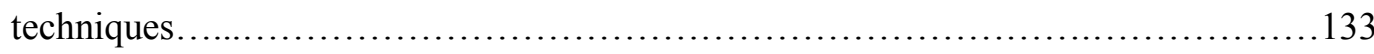

Table 28: LA-ICP-OES element emission lines for unprocessed cotton analysis...............137

Table 29: LIBS parameters for cotton analysis.....................................139

Table 30: LIBS element emission lines for unprocessed cotton analysis.....................148

Table 31: Analytical performance of LIBS using IAEA V-9...........................149

Table 32: Analytical performance of LIBS using AZ2006............................ 149

Table 33: LIBS LODs for cotton analysis....................................... 149

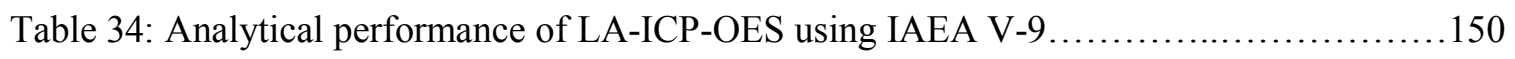

Table 35: Analytical performance of LA-ICP-OES using AZ2006...................... 150

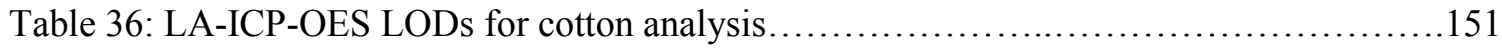

Table 37: Absolute detection limits for LA-ICP-OES and LIBS analysis of unprocessed

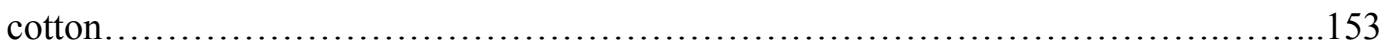

Table 38: Important aspects and performance requirements considered for instrument selection............................................................. 162

Table 39: LA-ICP-MS method for unprocessed cotton analysis............................ 165 
Table 40: LA-ICP-MS element menu for unprocessed cotton analysis. 166

Table 41: Analytical performance of LA-ICP-MS using IAEA V-9.

Table 42: Analytical performance of LA-ICP-MS using AZ2006. .167

Table 43: LA-ICP-MS LODs for unprocessed cotton analysis... .169

Table 44: Comparison of elemental analytical techniques.... .173

Table 45: Domestic cotton sample information for IRMS analysis.

Table 46: Foreign cotton sample information. 183

Table 47: Analytical performance of USGS-40 for $\delta^{13} \mathrm{C}$ analysis. 188

Table 48: Analytical performance of IAEA-CH-6 for $\delta^{13} \mathrm{C}$ analysis. 188

Table 49: Analytical performance of IAEA-601 and IAEA-602 for $\delta^{18} \mathrm{O}$ analysis 189

Table 50: Analytical performance of IAEA-CH-7 and coumarin for $\delta^{2} \mathrm{H}$ analysis. 190

Table 51: Comparison of sample performance when extensive sample preparation in employed.

Table 52: Sample information from frontier Spinning Mills 212

Table 53: Sample information from the Dumas, AR Cotton Classing Office. .213

Table 54: Cotton producing states organized by region. 215

Table 55: Elemental composition of several cotton bales from Post, TN

Table 56: LDA results of LIBS analysis of domestic cotton .227

Table 57: LDA results of LA-ICP-OES analysis of domestic cotton .227

Table 58: LDA results of LA-ICP-MS analysis of domestic cotton. .229

Table 59: LDA results for domestic and foreign cotton analysis by LIBS. .232

Table 60: LDA results for domestic and foreign cotton analysis by LA-ICP-OES .233

Table 61: LDA results for domestic and foreign cotton analysis by LA-ICP-MS. .235 
Table 62: LDA of all countries involved in transshipment..............................237

Table 63: LDA of all countries involved in transshipment-2 ..............................237

Table 64: LDA comparison of Chinese and Mexican cotton classification.....................237 


\section{LIST OF FIGURES}

FIGURE

PAGE

Figure 1: Typical LIBS setup, labels correspond to LIBS components listed in table 1...........9

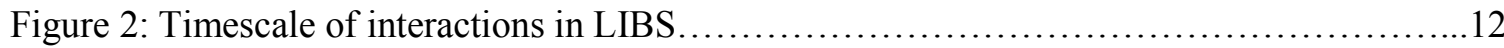

Figure 3: Mg response curves for NC2005; NC2005 is the first data point...................26

Figure 4: Electron density vs. laser Power density for $532 \mathrm{~nm}$ and $1064 \mathrm{~nm}$ LIBS analysis. The dotted line corresponds to the crossover point in analytical performance...............27

Figure 5: Resulting cotton crater as the number of laser shots increase. The change in crater diameter and depth as the number of repetitive laser shots in a single location is increased

............................................................................... 30

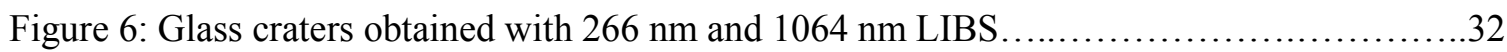

Figure 7: 3D cotton crater obtained using the same lens setup previously reported for glass analysis. The IR analysis of unprocessed cotton generated a $125.9 \mu \mathrm{m}$ diameter, 149.4 $\mu \mathrm{m}$ depth crater.

Figure 8: Cotton crater development using different laser irradiation wavelengths...............33

Figure 9: Day-to-day variation in ICP-OES response based on analyte IP....................43

Figure 10a: Typical linear range achieved for different plasma views in ICP-OES .............47

Figure 10b: Sensitivity ranges for different views of the ICP-OES plasma ...................47

Figure 11: Schematic of ICP-OES sample introduction system...........................50

Figure 12: Atomic emission line response in the presence of matrix constituents..............53

Figure 13: Ionic emission line response in the presences of matrix constituents...............53

Figure 14: Change in electron density in the presence of different matrix constituents..........54

Figure 15: Change in electron density in the presence of EIEs and NEIEs....................55

Figure 16: ICP-OES relative intensity trends in relation to $1^{\text {st }} \mathrm{IP}$ in the presence of $\mathrm{Zn} \ldots \ldots \ldots . .56$

Figure 17: ICP-OES relative intensity trends in relation to $2^{\text {nd }}$ IP in the presence of $\mathrm{Zn}$..........57

Figure 18: Filter setup for determining particle size effects in LA-ICP-OES ..................72

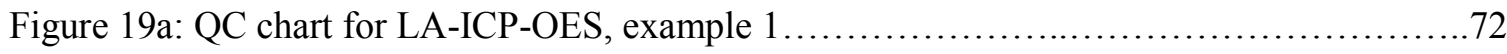

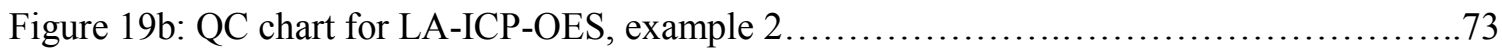


Figure 20: Transient signal integration for glass data interpretation

Figure 21: Maximum intensity observed with different gas composition and flow rates in LA-

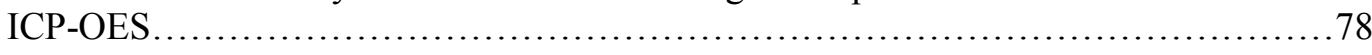

Figure 22: Transient signal for Mg in NC2005 using different flow rates and gas compositions..81

Figure 23: Transient signal for Mn in NC2005 using different flow rates and gas compositions..82

Figure 24: Transient signals in glass for elements of varying concentrations..................84

Figure 25: NC2005 Mg transient signals obtained with the filter device......................86

Figure 26: NC2005 Mn Transient signals obtained with the filter device.......................86

Figure 27: NC2005 Sr transient signals obtained with the filter device........................87

Figure 28: Effect of tubing length on transient signal................................... 89

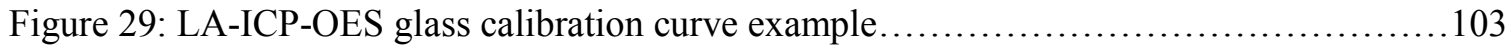

Figure 30: 3D glass crater obtained with LA-ICP-OES method; $100 \mu \mathrm{m}$ in diameter by $92.8 \mu \mathrm{m}$ depth. This translates to $\sim 600 \mathrm{ng}$ removed.................................... 106

Figure 31: Cotton boll and fiber composition....................................... 123

Figure 32: Heterogeneity observed in large batches of cotton by LIBS ........................128

Figure 33: SEM imaging of cotton powder; IAEA V-9 powder prior to any milling (left). New Mexico cotton following 20 minutes of milling (right) ........................... 130

Figure 34: Unprocessed cotton pellet............................................131

Figure 35a: NC2005 calibration curves generated with LIBS and LA-ICP-OES, LIBS example

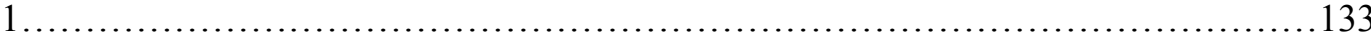

Figure 35b: NC2005 calibration curves generated with LIBS and LA-ICP-OES, LIBS example

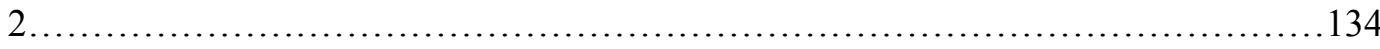

Figure 35c: NC2005 calibration curves generated with LIBS and LA-ICP-OES, LA-ICP-OES

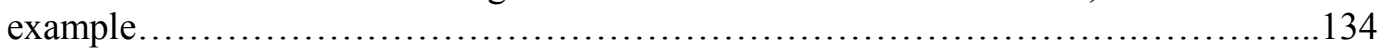

Figure 36: Comparison of concentration obtained in LIBS using peak intensity and peak area

Figure 37: Setup for argon introduction in LIBS...................................... 138

Figure 38a: SRM NIST 1411 performance using 1064 nm LIBS...........................139

Figure 38b: Day-to-day sensitivity obtained with LIBS using SRM NIST $1411 \ldots \ldots \ldots \ldots \ldots \ldots . . . . .140$

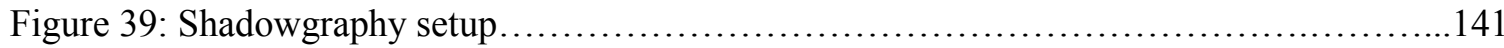


Figure 40a: Flashlamp and q-switch timing for shadowgraphy

Figure 40b: Enlarged q-switch region.

Figure 41: 1064 nm LIBS shockwave obtained in different environments. 144

Figure 42: $1064 \mathrm{~nm}$ LIBS shockwave obtained following ablation of unprocessed cotton.......145

Figure 43: 1064 nm LIBS shockwave morphology as cotton crater depth increases.............146

Figure 44: Comparison of 1064 nm LIBS shockwaves for pellets of different color.

Figure 45: Typical LA-ICP-OES cotton crater; 3D cotton crater obtained with LA-ICP-OES (left). Side slice of the crater taken at its deepest point (right).

Figure 46: Typical LIBS cotton crater; 3D cotton crater obtained with LIBS (left). Side slice of

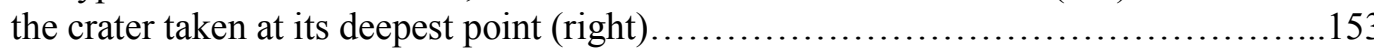

Figure 47: LIBS PCA of cotton test sample set, red = southeast U.S., black = southwest U.S...157

Figure 48: LIBS PCA of cotton test sample set - emphasis of emission line selection, black =

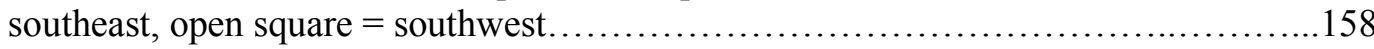

Figure 49: LA-ICP-OES PCA of cotton test sample set, red = southeast, black = southwest.....159

Figure 50a: LA-ICP-MS calibration curves using NC2005, example $1 \ldots \ldots \ldots \ldots \ldots \ldots \ldots \ldots \ldots . \ldots \ldots$

Figure 50b: LA-ICP-MS calibration curves using NC2005, example 2.......................168

Figure 51: Typical LA-ICP-MS cotton crater; 3D cotton crater obtained with LA-ICP-OES (left).

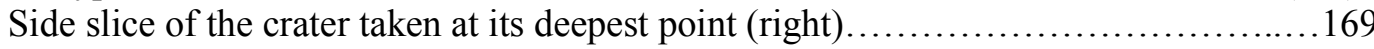

Figure 52: LA-ICP-MS PCA of cotton test sample set; black = southwest, red = southeast $\ldots . .170$

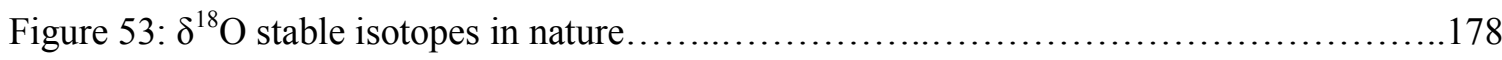

Figure $54: \delta^{13} \mathrm{C}$ in domestic unprocessed cotton.................................. 192

Figure $55: \delta^{13} \mathrm{C}$ in foreign unprocessed cotton....................................... 192

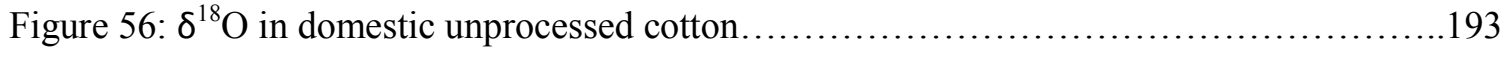

Figure $57: \delta^{18} \mathrm{O}$ in foreign unprocessed cotton....................................... 194

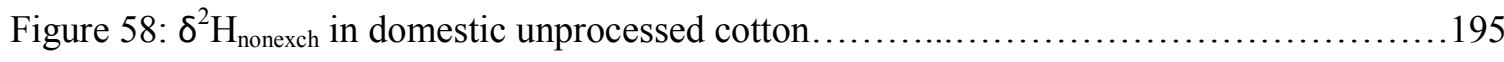

Figure 59: $\delta^{2} \mathrm{H}_{\text {nonexch }}$ in foreign unprocessed cotton....................................... 195

Figure 60: Relationship between $\delta^{2} \mathrm{H}_{\text {nonexch }}$ and $\delta^{18} \mathrm{O}$, black $=$ U.S., red $=$ China, orange $=$ Mexico, yellow $=$ Tanzania, green $=$ Pakistan, pink $=$ India and gray $=$ Brazil................196 
Figure 61: PCA of domestic and foreign unprocessed cotton analysis by IRMS; black = U.S., red $=$ China, orange $=$ Mexico, yellow $=$ Tanzania, green $=$ Pakistan, pink $=$ India and gray $=$ Brazil

Figure 62: Eigenvectors for variables utilized in figure 61 PCA; Y $=\mathrm{PC} 2, \mathrm{X}=\mathrm{PC} 1 \ldots \ldots \ldots \ldots 198$

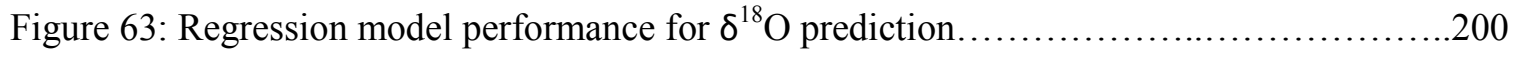

Figure 64: Regression model performance for $\delta^{2} \mathrm{H}_{\text {nonexch }}$ prediction.......................201

Figure 65: Schematic to conducting interpretation of cotton data..........................204

Figure 66: Simplified visual explanation of univariate statistics...........................205

Figure 67: Simplified visual explanation of multivariate statistics........................206

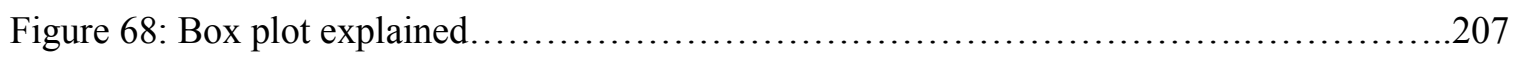

Figure 69: Schematic of LDA approach developed and utilized in this research...............216

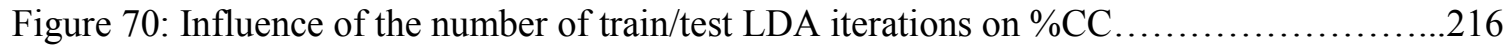

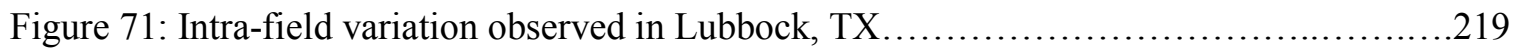

Figure 72 : Influence of harvest method on elemental profile.............................221

Figure 73: Variation within a field for different cotton varieties...........................222

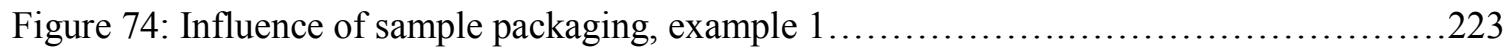

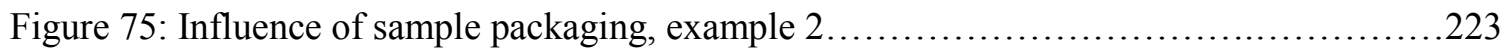

Figure 76: Scatterplot matrix used to estimate bivariate normality .........................225

Figure 77: PCA of all domestic cotton analyzed by LIBS, blue $=$ southwest, black $=$ southeast 226

Figure 78: PCA of all domestic cotton analyzed by LA-ICP-OES, blue = southwest, black=

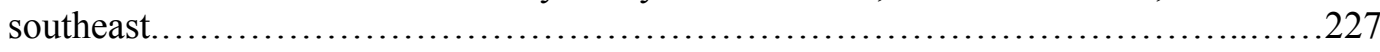

Figure 79: PCA of all domestic cotton analyzed by LA-ICP-MS, blue = southwest, black= southeast.

Figure 80: Geographic representation of $[\mathrm{Mg}]$ obtained in cotton grown throughout the cotton

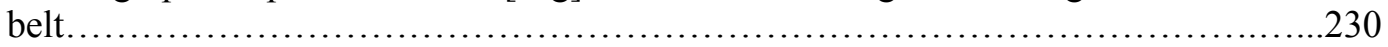

Figure 81: Geographic representation of [Sr] obtained in cotton grown throughout the cotton

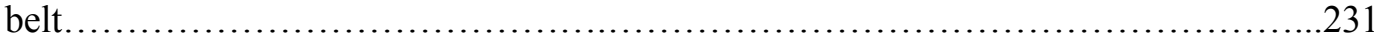

Figure 82: PCA of domestic and foreign cotton analyzed by LIBS. US = black, China = red, Mexico $=$ orange, Brazil $=$ yellow, Guadalupe $=$ dark green, India $=$ light green, Senegal $=$ blue, Pakistan $=$ teal, Tanzania $=$ purple and Zimbabwe $=$ pink. 
Figure 83: PCA of domestic and foreign cotton analyzed by LA-ICP-OES. U.S. = black, China $=$ red, Mexico $=$ orange, Brazil $=$ yellow, Guadalupe $=$ dark green, India $=$ light green, Senegal $=$ blue, Pakistan $=$ teal, Tanzania $=$ purple and Zimbabwe $=$ pink

Figure 84: PCA of domestic and foreign cotton analyzed by LA-ICP-MS. U.S. = black, China $=$ red, Mexico $=$ orange, Brazil $=$ yellow, Guadalupe $=$ dark green, India $=$ light green, Senegal $=$ blue, Pakistan $=$ teal, Tanzania $=$ purple and Zimbabwe $=$ pink $\ldots \ldots \ldots \ldots \ldots . .235$

Figure 85: Influence of cations on oxygen fractionation in water where the rate of the reaction is

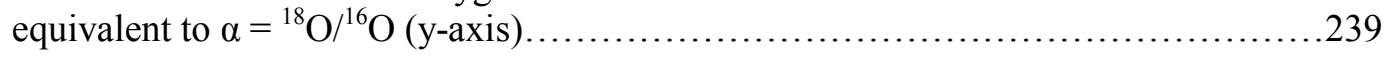

Figure 86: Correlation of $[\mathrm{Ba}]$ to $\delta^{18} \mathrm{O}$ in cotton. Black $=$ U.S., red $=$ China, orange $=$ Mexico, yellow $=$ Tanzania, green $=$ Pakistan, pink $=$ India and gray $=$ Brazil $\ldots \ldots \ldots \ldots \ldots \ldots 240$

Figure 87: PCA of Elemental and stable isotope results to determine additional trends pertaining to geographic region. Black $=$ U.S., red $=$ China, orange $=$ Mexico, yellow $=$ Tanzania,

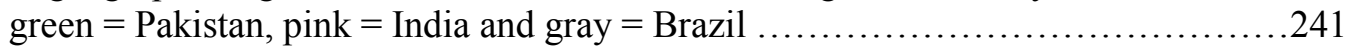

Figure 88: Regression model performance for $[\mathrm{Fe}]$ determination........................... 242 


\section{LIST OF ABBREVIATIONS AND ACRONYMS}

\begin{tabular}{|c|c|}
\hline ANOVA & American Society for Testing and Materials \\
\hline ASTM & Continuing calibration verification \\
\hline $\mathrm{BEC}$ & Background equivalence concentration \\
\hline $\mathrm{CCD}$ & Charge-coupled device \\
\hline $\mathrm{CCV}$ & Analysis of variance \\
\hline CTD & Charge transfer device \\
\hline DA & Discriminant analysis \\
\hline EIEs & Easily ionized elements \\
\hline FOC & Fiber optic cable \\
\hline FGS & Float glass standard \\
\hline HALC & Height above load coil \\
\hline IAEA & International Atomic Energy Agency \\
\hline iCCD & Intensified charge coupled device \\
\hline IP & Ionization potential \\
\hline IR & Infrared \\
\hline IRMS & Isotope ratio mass spectrometry \\
\hline ISO & International Organization for Standardization \\
\hline LA-ICP-AES & Laser ablation inductively coupled plasma atomic emission spectrometry \\
\hline LA-ICP-MS & Laser ablation inductively coupled plasma mass spectrometry \\
\hline LA-ICP-OES & Laser ablation inductively coupled plasma optical emission spectrometry \\
\hline LDA & Linear discriminant analysis \\
\hline LDF & Linear discriminant function \\
\hline LDR & Linear dynamic range \\
\hline LIBS & Laser-induced breakdown spectroscopy \\
\hline
\end{tabular}




\begin{tabular}{|c|c|}
\hline LIPs & Laser-induced plasmas \\
\hline LOD & Limit of detection \\
\hline LOQ & Limit of quantitation \\
\hline LTSD & Lens-to-sample distance \\
\hline LTE & Local thermodynamic equilibrium \\
\hline MRS & Mylnefield Research Services \\
\hline NAA & Normal analytical zone \\
\hline NAZ & Neutron activation analysis \\
\hline Nd:YAG & Neodymium-doped yttrium aluminum garnet \\
\hline NIST & National Institute of Standards and Technology \\
\hline NEIE & Non-easily ionizable element \\
\hline PCA & Principal component analysis \\
\hline QC & Quality control \\
\hline RBTN & Regional Breeders Testing Network \\
\hline REEs & Rare earth elements \\
\hline RF & Radio frequency \\
\hline RI & Refractive index \\
\hline RSD & Relative standard deviation \\
\hline SA & Self-absorption \\
\hline SCD & Segmented-array charge-could device \\
\hline SEM & Scanning electron microscopy \\
\hline SEM/EDS & Scanning electron microscopy/energy dispersive spectroscopy \\
\hline SNR & Signal-to-noise ratio \\
\hline SRM & Standard reference material \\
\hline XRF & $\mathrm{X}$-ray fluorescence \\
\hline
\end{tabular}




\subsection{INTRODUCTION}

\subsection{Current State of the Problem}

The research conducted within the scope of this dissertation strives to provide a method for detecting fraudulent unprocessed cotton in the US textile market. Cotton is the most abundant natural fiber in the world ${ }^{1}$. Many countries, the U.S. included, are involved in the growing, importation, exportation and production of this commodity. Textile and apparel related imports in 2008 accounted for a mere $5 \%$ of the total imports to this country, but were responsible for $43 \%$ ( $\$ 11$ billion) of the entire duties collected at U.S. ports ${ }^{2}$. Paper documentation is the sole means of certifying the cotton variety and origin which is associated with cotton quality and, in turn the end application of this good. The paper documentation for cotton commodity imports can be easily falsified, making it essential to develop a reliable and independent method for corroborating the paperwork. Falsifying the country of origin is the most common and efficient method of committing fraud. Different regions are responsible for the production of different cotton varieties. Coupled with various trade agreements and quotas in place between the U.S. and other countries, this can result in quite different tariff assignments if the documented source of the imported good is inaccurate. Misclassification of cotton commodities due to the incorrect identification of source costs the U.S. millions of dollars in uncollected tariffs each year².

The following chapters describe the successful evaluation of elemental analysis and stable isotope ratios for geographically provenancing domestic and foreign grown cotton. Multivariate statistics are employed as a means of demonstrating and modeling the variation related to geographical regions that are utilized in this research. 


\subsection{Research Motivation and Proposed Solutions}

The scope of this research utilized laboratory analysis to solve an economical and industrial fraud problem. The following chapters explore the application of various elemental analysis techniques and the evaluation of stable isotope ratios as a means for determining the geographic source of unprocessed cotton.

It is hypothesized that the elemental signature and the stable isotope ratios of cotton commodities can provide an approach independent to paper documents to authenticate geographic source.

Although these analytical techniques are different in the analyte detected, it is hypothesized that they will yield complementary information characteristic of a particular geographic region.

Climate and soil nutrients vary by region. This research exploits the factors using the variation in nutrient uptake and metal content from the mineralogy of the soil constituents as well as stable isotope ratios related to climate information to associate a cotton sample to a geographic region.

Several elemental analysis techniques are being evaluated in order to determine the most simplistic, cost effective analytical tool for this forensic application. In particular, laser-induced breakdown spectroscopy (LIBS) is being explored as a less complex, potentially field portable technique - ideal for this application. Laser ablation sample introduction is being coupled to inductively coupled plasma optical emission spectrometry (LA-ICP-OES) to determine the applicability of this solid sampling approach for conducting analyses for forensic purposes. These

analytical tools will be evaluated as alternatives to the elemental analysis "gold standard"," laser ablation inductively coupled plasma mass spectrometry (LA-ICP-MS), to determine if comparable information can be obtained with less cumbersome, less expensive instrumentation.

In addition to evaluating LIBS for this particular application, this research also proposes that through investigations of plasma conditions and ablation processes a more standardized approach 
to this technique can be developed. There are several parameters that can be manipulated in a LIBS experiment (laser wavelength, laser power, laser focusing distance, spectrometer gate delay and width, etc.). Results from the research conducted herein aim to demonstrate that analytical LIBS optimization consists of some important parameters that are uniform regardless of the properties of the matrix. Studies presented in the dissertation will utilize different matrices in order to determine the extent to which widespread applicability is appropriate. Not only is this beneficial to the LIBS researcher, but also to the advancement of the commercialization of this technique. The studies presented address the advancement of LIBS by evaluating new applications and investigating means to simplify instrumental design.

The final aim of this research emphasizes the significance of applying multivariate statistical approaches to interpret and model the elemental and isotopic data. Within the LIBS community alone there are several statistical tools used to model data with no agreement as to the most appropriate data treatment method for assessing broadband spectroscopic data. The research evaluates currently reported modeling techniques in the literature in addition to developing new strategies to compare the data using multivariate statistics. These approaches are applied to other elemental analysis techniques to assess the overall usability for interpreting geographic trends. In addition, multivariate statistics are used to determine the relation, if any, between stable isotopes and elemental composition.

\subsection{Significance of Study}

For the first time geographic provenancing of unprocessed cotton has been accomplished from both an elemental analysis perspective and through the application of stable isotope ratios. The present work is important because it has real world applications relevant to forensic and fraud detection. 
In addition to the provenancing application, the current studies provide an in-depth look into LAICP-OES for forensic applications in particular, but also for probing scientific interest to determine the feasibility of coupling these two analytical tools. The importance of operating conditions and a method for mitigating matrix effects is presented in order to address the analytical significance of the technique. Because LA-ICP-OES has not been used as much as LAICP-MS, extensive explorations of important parameters for generating robust plasma conditions are warranted. The work presented here sets a precedent for LA-ICP-OES operating parameters, independent of matrix or laser ablation unit used. This research provides an important foundation for other scientific researchers or analysts in the community. In addition an extensive comparison between LA-ICP-OES and LA-ICP-MS is presented using float glass and unprocessed cotton, addresses analytical performance capabilities of this technique.

Lastly, this dissertation provides a non-experimental approach to simplifying analytical LIBS optimization. The research aids the advancement of LIBS instrumentation as the current studies suggest the use of generalized, universal operating parameters, simplifying the method development phase of the analysis. The knowledge gained herein supplies a more standardized approach to experimental LIBS, which is important for both the LIBS research and commercialization communities.

The research conducted within the scope of this dissertation has been organized according to the overall goals of each study. For the sake of clarity the research is presented as two components, both of which are important to the completion of this project. Part I includes all studies that pertain to fundamental interactions in the plasma and involve the application of these interactions to improve analytical performance of several techniques. Part II of this dissertation applies the knowledge gained in Part I to develop methods for forensic applications. Multivariate statistics 
are presented as a means to interpret the feasibility of utilizing these methods for the intended forensic related purpose.

\section{PART 1: STUDIES OF PLASMA INTERACTIONS IN ATOMIC EMISSION SPECTROSCOPY}

\subsection{STRATEGY FOR STANDARDIZATION OF ANALYTICAL LIBS METHODS THROUGH THE DEVELOPMENT OF UNIVERSAL PARAMETERS}

\subsection{Overview}

Laser-induced breakdown spectroscopy (LIBS) is an analytical technique that is increasing in popularity and expanding in diversity of applications. These applications span several fields with analysis being conducted on in-house instrumental setups as well as commercial instruments. Several parameters - pertaining to both the ability to achieve excitation and ionization (laser source) and to accomplish the detection of emission (spectrometer source) - can be changed or manipulated, resulting in complex method optimization studies. The ability to generate an optimized method influences the level of analytical performance that can be achieved and thus optimization is crucial, yet can be time consuming. The current studies address the exploration and make recommendations towards the adoption of universal LIBS parameters for some critical operating conditions. A comprehensive scheme is employed to explore the possibility of simplifying analytical LIBS methodology to yield a more standardized analytical technique by evaluating both laser and spectrometer parameters. Results are presented for several different matrices - cotton, glass and soil - different laser irradiation wavelengths, as well as both in-house and commercial instrumental setups. Similarity in trends stresses the widespread applicability of these universal parameters for $532 \mathrm{~nm}$ and $1064 \mathrm{~nm}$ LIBS experimentation. These studies combine plasma conditions and ablation processes in order to understand how these complex 
properties interact. A novel approach to depicting ablation efficiencies by means of an intensity / mass removed ratio is presented. Information from this measurement as well as estimates in electron density are used to determine a balance between plasma and ablation conditions to yield optimized parameters that are relevant to several matrices. The current studies imply that universal LIBS parameters exist independent of the physical properties of the target material.

\subsection{Introduction}

\subsubsection{Atomic Emission Spectroscopy}

An analytical chemist and a forensic scientist ask several questions regarding the identity of an unknown sample. The questions are meant to establish associations between different samples. Scientists use instrumental techniques to probe the unknown to determine important physical and chemical characteristics. The simplest questions pertaining to the comparison of known and unknown that can be addressed is "which elements are present and at what concentrations are they present?" The approach to analyzing a sample encompasses elemental analysis. The focus of the present chapter is to introduce elemental analysis by means of exploring atomic emission spectroscopy.

Atomic spectroscopic techniques involve the absorption or emission of electromagnetic radiation from atoms in a sample ${ }^{4}$. The emission process can either be spontaneous or stimulated. For emission to occur, other events must first take place.

Consider an atom; this atom is surrounded by discrete orbitals of electrons. The further the orbital is from the center of the atom, or nucleus, the higher the energy. It is important to remember that the electrons in the atom can move or travel about any of the orbitals. This is reflected in the state of the atom. When the electrons are at the closest to the nucleus this is the lowest energy state which can be achieved and is, in turn referred to as the ground state of the atom. The addition of energy to the atom can result in one of two things: 1) a change the kinetic energy of the atom or 
2) the energy can be absorbed by the atom ${ }^{4,5}$. The absorption of this energy results in the ground state atom becoming excited. Excitation involves an electron from an orbital in close proximity to the nucleus being promoted to a higher energy orbital further from the nucleus ${ }^{4,6}$.

This additional energy can be introduced to the atom in several ways:

1) Collision with an electron

2) Collision with another atom

3) Collision with an ion

4) Collision with a molecule

5) Absorption as a result of exposure to electromagnetic radiation

The state of excitation is not eternal. It is actually quite short-lived, mostly because the lower energy states are more stable than the higher energy levels. The decay, or return to ground state may be radiative or non-radiative. For the purpose of atomic emission techniques radiative decay is most important. Radiative decay involves the emission of a "particle" of electromagnetic radiation known as a photon ${ }^{4}$.

Although atomic emission techniques include the detection of emission of photons as excited atoms decay to ground state, analytical approaches also involve the decay following the excitation from a ground state of an ion. An ion is formed when an electron is completely removed from the atom following high energy excitation. The energy required for this is known as the ionization potential (IP); each element has several IPs, pertaining to the generation of different ionic states. Ions exist with a positive net charge. They have both excited and ground states and undergo similar emission processes previously described. Both emission from ionization and excitation can be detected using atomic emission techniques. 
The radiative decay, whether from an ion or an atom, occurs across finite energy levels. The decay corresponds to characteristic wavelengths of light that can be attributed to particular energy transitions, and, in turn, an element. The relationship between energy and wavelength can be described by Planck's equation:

$$
E=h v=\frac{h c}{\lambda}
$$

where lambda ( ) corresponds to the wavelength of the atomic emission line. The wavelengths of light that are utilized in atomic emission occur between 150-900 $\mathrm{nm}$.

In order to conduct atomic analysis the substance in question must somehow be decomposed to its atomic constituents. Atomic emission techniques relevant to this research employ a plasma, or ionized gas to breakdown the material. The research presented here includes both the application of laser-induced plasmas (LIPs) as well as introducing the sample into a continuous plasma to achieve ionization, excitation and subsequent detection. In particular, LIBS is the extensive LIP technique utilized within the scope of this research. This technique will be discussed further in section 2.2.2.1. The continuous plasma source utilized in this research is the product of inductively coupling. Inductively coupled plasma optical emission spectrometry (ICP-OES) is the continuous plasma source employed in this research. This technique is discussed in more detail in section 3.2.1. Both of these atomic emission techniques are capable of multi-element analysis.

\subsubsection{LIBS}

The concept of focusing a laser pulse to produce high irradiation of a matrix of solid, liquid or gas composition for spectrochemical analysis was first introduced in 1962 by Brech and Cross ${ }^{7}$. This principle has advanced into the development of analytical techniques as well as industrial applications utilizing plasmas. The LIBS approach uses a short (femto to nanosecond range) 
duration laser pulse of sufficient energy to interact with the matrix to yield ablation (in solids), followed by thermal breakdown (vaporization), excitation and ionization ${ }^{8}$.

The analytical technique was first introduced in the 1960s with several advancements in lasers as well as innovations in the analyses conducted and detection strategies. Detailed accounts of the history and progression of LIBS can be found elsewhere ${ }^{8-10}$. A typical LIBS setup is depicted in figure one.

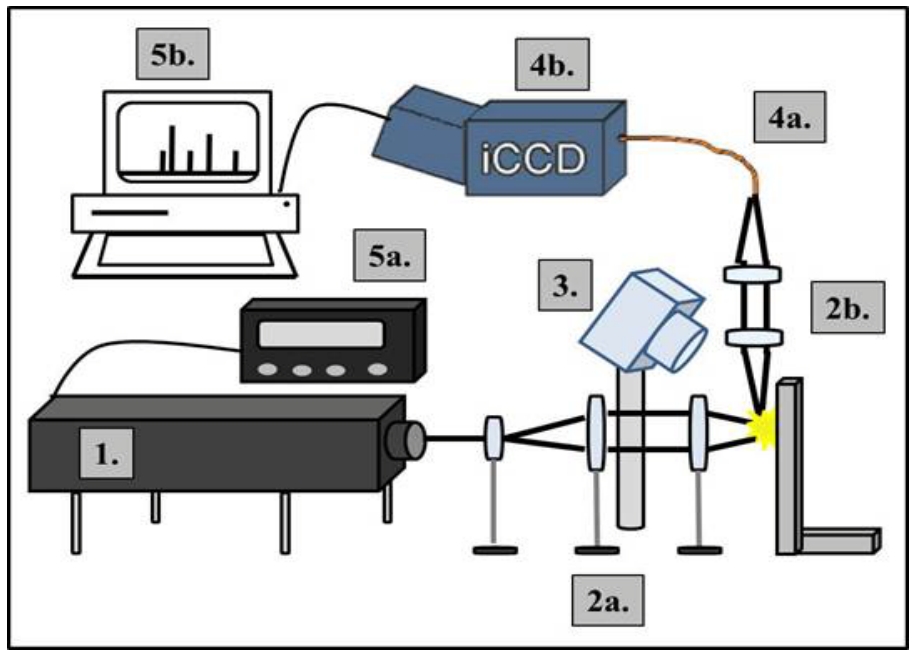

Figure 1: Typical LIBS setup, labels correspond to LIBS components listed in table 1.

Table 1: Components of a generic LIBS system

\begin{tabular}{|rl|}
\hline \multicolumn{2}{|l|}{ LIBS Components } \\
\hline 1. & Laser \\
\hline 2. & Optics \\
\hline & 2a. Laser focusing optics \\
\hline & 2b. Plasma light optics \\
\hline 3. & Camera \\
\hline 4. & Detection System \\
\hline & 4a. Fiber optic cable \\
\hline & 4b. Spectrometer \\
\hline 5. & Electronics \\
\hline & 5a. Delay generator \\
\hline & 5b Computer processor \\
\hline
\end{tabular}


Although several types of lasers have been reported in the literature for LIBS, this research employs a solid state laser, neodymium-doped yttrium aluminum garnet $\left(\mathrm{Nd}: \mathrm{Y}_{3} \mathrm{Al}_{5} \mathrm{O}_{12}\right.$ or Nd:YAG). The Nd:YAG lasers are the most common used LIBS laser. It utilizes a crystal to produce the lasing medium activity ${ }^{11}$. The host crystal for this class of lasers is in the YAG component. Approximately $1 \%$ of the yttrium ions are replaced with triply ionized neodymium Nd (III) ions ${ }^{11}$.

The Nd:YAG lasers emit light at $1064 \mathrm{~nm}$, which is considered the fundamental optical wavelength. Other wavelengths can be achieved by using coated optics within the laser cavity which can double the frequency to generate laser emission at $532 \mathrm{~nm}$ or other $\mathrm{Nd}: \mathrm{YAG}$ harmonicas like $355 \mathrm{~nm}$ and $266 \mathrm{~nm}$.

The Nd:YAG lasers are typically used in LIBS because they are less expensive than other lasers and also because these laser are capable of producing high energy, short pulses using a mechanism called q-switching. In the simplest of terms, q-switching makes use of an optical switch that determines when the maximum or saturation of the population inversion has occurred prior to opening and releasing a high energy laser output ${ }^{12}$.

Optical lenses coated to provide optimal transmission of particular wavelengths of light (UV, Vis, NIR or IR) are used to expand, collimate and focus the laser beam. The approach of expanding the beam prior to focusing, yields a more narrow beam waist then what can be achieved with focusing alone.

The plasma light is collected using fused silica lenses that also collimate and focus the light, however for this particular instance the light is focused onto a fiber optic cable (FOC). The FOC is used to transport the light to a spectrometer, which is able to separate it into wavelengths and correlate the amount of light to an intensity value. Several spectrometers can be used for detecting 
the emission produced in an analytical LIBS experiment. However, the research conducted used an intensified charge coupled device (iCCD) so this spectrometer will be discussed. An intensifier coupled to a CCD is used to amplify the light signal collected to provide more sensitive measurements.

A LIP, especially one produced in an analytical LIBS experiment is short lived, decaying over microseconds. Because of the timescale of LIBS plasma evolution an external gating device that controls the laser and spectrometer so that the collection of the atomic emission can be achieved at its optimum.

\subsubsection{The Physics of LIBS}

The short-lived plasma is characterized by particular interactions between plasma species that occur across several time regimes. The brightest most extreme intense light is observed initially during the evolution of the plasma. This is known as the continuum. The continuum is the result of bremsstrahlung and recombination events ${ }^{13}$. These events from the early time regime occur between electrons or electrons and ionic or atomic species. The bremsstrahlung process is described by the emission of a photon as free electrons collide ${ }^{8,13}$. When an electron is captured or recombined by an ionic or atomic species this is considered a combination event ${ }^{8}$.

Once the fleeting continuum has decayed so that it does not predominate in the plasma, ions, atoms and eventually molecules decay and emit characteristics wavelengths of light. The order of decay is based on the stability of the higher energy levels; the less stable resulting in a shorter lifetime in the plasma.

From the point in time in which the laser makes contact with the sample, until several microseconds after, the plasma expands outwards in all possible directions not obstructed by the sample itself. The speed of the plasma expansion is of such magnitude that a shockwave is 
produced and can be visually observed under specific conditions. The shockwave has been studied and has been shown to be indicative of the plasma morphology ${ }^{14}$.

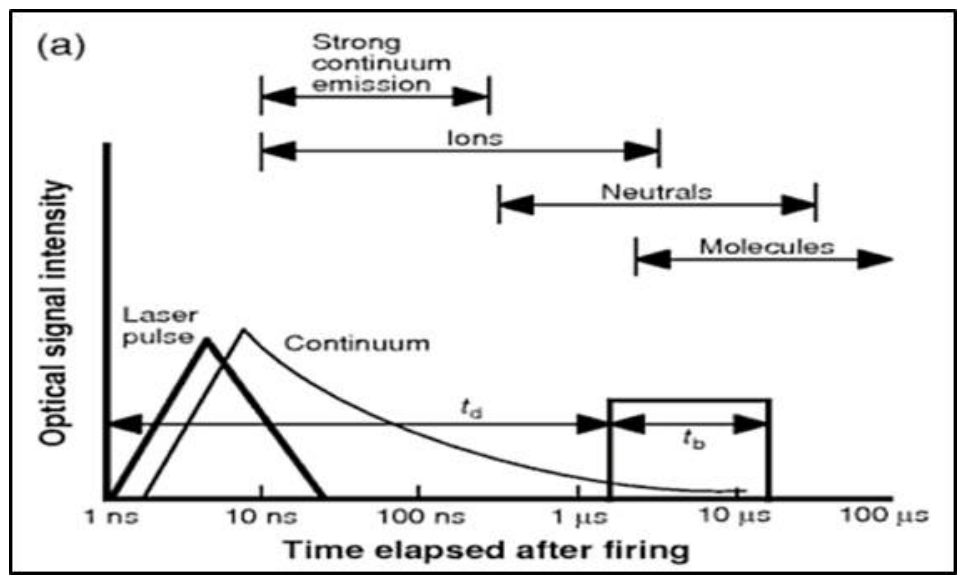

Figure 2: Timescale of interactions in LIBS99.

In addition to visual inspection of the plasma, there are several other means to investigate the dynamics and interactions taking place in this space and time regime. The species within the plasma can vary in their distribution across different degrees of ionization and excitation. To describe these population distributions the Saha and Boltzmann equations can be used. The Saha equation is a theoretical approach to determining the distribution of species or ions in different states of ionization ${ }^{5}$. The Boltzmann equation, on the other hand, can be used to calculate the differences in distributions for species in excited states ${ }^{5}$. These equations or models do not only consider the population distributions but can also be used to solve for the ionization and excitation temperatures in the plasma as well. This is important because different energy states are not only governed by the obvious - differences in energy, but the populations of these states also depend on temperature. At higher temperatures the upper energy state predominates over the lower energy. As the temperature decreases the distribution reverses. The Saha equation can be described by the following: 


$$
\frac{n_{i} n_{e}}{n_{a}}=\left(\frac{2 \pi m_{e} k T}{h^{2}}\right)^{3 / 2}\left(\frac{2 Z_{i}}{Z_{a}}\right) \exp \left(\frac{-E_{i}}{k T}\right)
$$

where subscripts "a" and "i" correspond to variables relating to the atomic and ionic states, respectively. The " $n$ " terms refer to concentrations of the two states, with the exception of $n_{e}$ which is the electron density. Other variables include the mass of an electron, $m_{e}$, and the ionization potential $\mathrm{E}_{\mathrm{i}}$ of the species. The temperature calculated here is $\mathrm{T}_{\mathrm{ion}}$ or the ionization temperature.

The Boltzmann equation can be described by the following:

$$
\frac{N_{1}}{N_{0}}=\left(\frac{g_{1}}{Z}\right) \exp \left(\frac{-E_{1}}{k T}\right)
$$

for the relationship with a species in the ground state, where $\mathrm{Z}$ corresponds to the partition function of the ground state ${ }^{8}$. This can also be calculated with respect to a relationship that does not involve a ground state species. In that case, $\mathrm{Z}$ is replaced by the statistical weight of the lower energy state.

An additional method can be used to calculate the plasma temperature relevant to atomic and ionic species. This method is known as the two-line $\operatorname{method}^{8}$. This approach is described in equation 4:

$$
\frac{I^{\prime}}{I}=\left(\frac{\lambda g^{\prime} A^{\prime}}{\lambda^{\prime} g A}\right) \exp \left[-\left(E^{\prime}-E\right) / k T\right]
$$

where "g" refers to the statistical weights of the species and " $\mathrm{A}$ " the transition probabilities. Line selection is typically determined by the availability of values for $\mathrm{g}$ and $\mathrm{A}$, which are reported in the National Institute of Standards and Testing (NIST) Atomic Line Database ${ }^{15}$. In addition, 
spectral lines should be of approximately the same width, this is used to assume similar effects of broadening and detector response so as not to influence the calculation.

The spectral line ratio approach was used in this research to evaluate the plasma characteristics for experiments discussed in this chapter. The most common investigative measurements of the quality of the plasma involve determining the electron density and the absolute plasma temperature. In LIBS experiments plasma temperatures typically less than $12,000 \mathrm{~K}$ following the decay of the continuum ${ }^{8}$. The temperature calculated above can then be used to determine the electron density. The electron density in this research has been calculated by solving for $\mathrm{n}_{\mathrm{e}}$ in the following equation:

$$
\frac{I_{\text {ionic }}}{I_{\text {atomic }}}=\frac{4.83 \times 10^{15}}{n_{e}} \times\left(\frac{g_{q} A_{p q}}{\lambda_{p q}}\right)_{\text {ionic }} \times\left(\frac{\lambda_{p q}}{g_{p} A_{p q}}\right)_{\text {atomic }} \times T^{3 / 2} \exp \frac{-\left(E_{i}+E_{q}+E_{p}\right)}{k T}
$$

where

$$
4.83 \times 10^{15}=\frac{2 \pi m_{e}}{h^{2}}
$$

is used for simplification of constant terms. Most of the terms utilized in this expression have been previously defined, with the exception of $E_{i}, E_{q}$ and $E_{p}$ which correspond to the first ionization potential of the element being evaluated, the energy of the upper state $q$ and the energy of the lower state $\mathrm{p}$.

The ability to use this approach relies on the assumption that the plasma exists in local thermodynamic equilibrium (LTE). The term "local" is included because in the plasma thermodynamic equilibrium exists in small, localized regions. The state of LTE will vary from region to region within the plasma ${ }^{8}$. 


\subsubsection{Introduction to Analytical LIBS and the Need for Standardization in Operating Conditions}

Analytical LIBS involves the spectrochemical analysis of solids, liquids, aerosols or gases in order to determine qualitatively or quantitatively the elements present in a particular matrix. The accomplishment of this task encompasses the optimization of several parameters related to the properties of the laser and the spectrometer in order to achieve optimal emission detection.

Typically several optimization studies are carried out to determine suitable working conditions for experimental analysis. Important parameters are listed and described in table 2.

Table 2: Important parameters in LIBS optimization and method development

\begin{tabular}{|c|c|}
\hline LIBS Parameter & Rationale \\
\hline \multicolumn{2}{|l|}{ Laser Related Parameter } \\
\hline Laser wavelength & Reflects the quality of laser-sample interaction \\
\hline Ablation mode (spot vs. line) & $\begin{array}{l}\text { Surface analysis vs. depth profiling; size of sample } \\
\text { could be a determining factor }\end{array}$ \\
\hline Energy & $\begin{array}{l}\text { Influenced by physical properties of the matrix } \\
\text { (breakdown threshold) }\end{array}$ \\
\hline Duration of ablation & $\begin{array}{l}\text { Influenced by physical properties of the matrix; } \\
\text { mass removal with each shot and sensitivity } \\
\text { required for analysis }\end{array}$ \\
\hline \multicolumn{2}{|l|}{ Detection Related Parameters } \\
\hline Gate delay $(\mu \mathrm{s})$ & $\begin{array}{l}\text { Time required post laser-sample interaction to } \\
\text { detect emission related processes }\end{array}$ \\
\hline Gate width $(\mu s)$ & $\begin{array}{l}\text { Time window for detected emission processes, } \\
\text { varies with matrix composition }\end{array}$ \\
\hline
\end{tabular}

Analytical LIBS is an elemental analysis technique that has been reported for a variety of applications traversing forensic ${ }^{16,17}$, biological ${ }^{18}$ and industrial ${ }^{19}$ fields. The process of laser ablation is extremely complex ${ }^{20}$ and is influenced by several parameters of the selected laser. In addition, following the generation of a LIP, the ability to detect the emission processes with sufficient sensitivity relies on the selection and optimization of several parameters related to the spectrometer. For successful analytical LIBS analysis, it is essential to optimize parameters that 
are associated with both the laser and spectrometer. The selection of certain parameters as described in table 2, influence plasma conditions and are necessary to reduce the influence of continuum radiation ${ }^{13}$ and to improve the quality or precision of the analysis.

The ability to accomplish that which has been described above relies on the basis of conducting method optimization. In LIBS analysis, the ultimate goal of optimization is to yield maximum emission signals, with the lowest signal-to-noise ratios (SNR), and to do so with the best possible precision $^{21}$. This can often be a difficult, complex process. Factors like laser wavelength and spectrometer type are often dictated by the instrumental configuration. These parameters have a direct influence on the analytical capabilities that can be achieved. The variety in parameters even before LIBS optimization (laser and spectrometer selection), stress the need to evaluate LIBS for potential universal or standardization in parameters to reduce the complexity in analytical measurement and performance of the technique.

Previous work has considered the need for consistency in optimization, steel selected as a model matrix. This was accomplished by determining the relationship between ablation efficiency (defined as crater depth per unit fluence) and plasma lifetime ${ }^{21}$. However, this study considered primarily the influence of laser fluence on achieving the desired analytical performance.

Although laser fluence is often reported in the literature, the use of power density as a method of describing laser parameters takes into account the factor of time. The pulse duration has been shown to influence the ablation process ${ }^{22}$, producing a deeper crater and in-turn indirectly affecting analytical measurements. In order to more clearly assess trends on the basis of laser parameters, power density will be employed in the current studies presented here.

In addition, the ability to effectively remove particles from the target using laser ablation, so as to vaporize and ionize the material, relies directly on the laser output. High irradiation results in plasma temperature and electron density gradients as the ablation surface transforms into a crater or cavity $^{23}$, and has the potential to induce mass loading effects as the distribution of particles into 
the plasma changes ${ }^{24}$. With the use of a higher laser output, a hotter, denser plasma is generated. Higher laser output also influences the quality of laser-sample interactions, a "roll-off 25 " or decrease in the ablation efficiency has also been observed. These reports demonstrate that the selection of laser parameters has the potential to negatively reflect on the analytical performance. The aim of this chapter is to evaluate perspective universal parameters for analytical LIBS studies. By determining a balance between plasma processes and analytical conditions a more efficient LIBS method can be developed.

The ability to make assessments of ablation efficiency and ablation rate as these factors pertain to power density results in an improved understanding in the analytical measurements conducted using LIBS in addition to the fundamental behaviors in the plasma. Similar studies conducted by LA-ICP-MS have also considered the implications of some of these factors for several matrices ${ }^{26}$. Although a different mode of detection, both techniques use laser ablation and thus similar trends regarding the influence of laser parameters on properties of mass removal are expected. In both instances small, uniform ablated particles yield better analytical results.

Different matrices, including cotton, glass and soil, are compared in this work to thoroughly assess the applicability of universal parameters for analytical LIBS of different matrices. The matrices considered have different physical properties which are reflected in the responses to ablation. Comparisons are made using different laser irradiation wavelengths $(266 \mathrm{~nm}, 532 \mathrm{~nm}$ and $1064 \mathrm{~nm}$ ), the configuration of the LIBS setup (in-house or commercial system) as well as the influence of ablation related processes to make inferences. Ablation processes are addressed by determining relationships between crater aspect ratio, analytical measurement and mass removal estimates. The parameters are used in parallel with plasma conditions to explain similarities in LIBS method parameters for the different matrices. 


\subsection{Materials and Methods}

\subsubsection{Description of Samples and Sample Preparation}

A variety of matrices have been included in the studies in order to address the ability to overcome differences in physical properties between matrices to achieve uniformity in some important LIBS conditions. The matrices vary in important characteristics like color, latent heats of vaporization and heat capacity, which influence laser-sample interactions and ablation processes.

\subsubsection{Cotton Matrix}

The cotton utilized in these studies consists of cotton prior to any step in the manufacturing process, also referred to as unprocessed $\operatorname{cotton}^{27}$. The cotton samples used in this study consist of an in-house cotton standard. The standard was developed and utilized in this work is from a 2005 North Carolina harvest (abbr. NC2005) and has been thoroughly characterized in several batches by LA-ICP-MS and LIBS in order to determine an accurate representation of baseline concentrations of $\mathrm{Al}, \mathrm{Ba}, \mathrm{Ca}, \mathrm{Fe}, \mathrm{K}, \mathrm{Mg}, \mathrm{Mn}, \mathrm{Na}$ and $\mathrm{Sr}$, section 6.3.1.4. The sampling strategy and preparation of the unprocessed cotton will be presented later, sections 6.3.1.1-4, accompanying the analytical analysis of cotton for geographic provenancing.

\subsubsection{Glass Matrix}

Several NIST glass (NIST, Gaithersburg,MD, USA) standard reference materials (SRMs) were used in the studies. All glass SRMs utilized in these studies were of full thickness. Glass SRMS used were NIST 610, NIST 612, NIST1411 and NIST 1831. These glasses vary not only in concentration but also in color and transparency. The differences in samples were used to evaluate the laser-sample interactions that could be influenced by different physical properties within a particular matrix. 


\subsubsection{Soil Matrix}

Soil SRMs were also used as an additional matrix for this analysis. The soil was prepared and pelleted according to a previously described method $^{17}$. The soil standards consisted of different soil types and varied from low to extremely elevated concentrations for several elements. The specific three SRMs included were NIST 2704 (Buffalo River Sediment, NIST), NIST 2710 (Montana I Soil, NIST) and PACS-2 (Marine River Sediment Reference Material, National Research Council, Canada).

\subsubsection{LIBS Experimental Design}

\subsubsection{UV (266 nm) LIBS In-house Setup}

An ultraviolet (UV) LIBS setup similar to what has been previously reported ${ }^{3,17}$ has been used. A 266 nm q-switched Nd:YAG Tempest laser (New Wave, Fremont, CA) was used as the ablation and excitation source. A maximum laser pulse of $29 \mathrm{~mJ}, 4 \mathrm{~ns}$ in duration, was first expanded and collimated prior to focusing the laser using a plano-convex lens, $50.8 \mathrm{~mm}$ diameter, $\mathrm{f}=150 \mathrm{~mm}$. The focusing lens was fixed on a micrometer, allowing for adjustments to be made in where the laser was focused in respect to the sample surface, allowing the lens-to-sample distance (LTSD) to be changed with ease for different matrices. The surface of the sample and crater morphology was both monitored using a camera equipped with a zoom lens. A consistent LTSD could be ensured using this method. The plasma light emission was collected at $90^{\circ}$ to the irradiation source using two fused silica lenses $\mathrm{f}=75 \mathrm{~mm}$ to collimate and focus the light on a $50 \mu \mathrm{m}$ FOC. The light was transferred to a Mechelle 5000 spectrometer equipped with an iCCD iStar camera (Andor Technologies, South Windsor, CT). The spectrometer setup has a resolving power of 5000. The LIBS setup was controlled using a digital delay generator model 656 (Berkeley Nucleonics, San Rafael, CA, USA). 
The glass samples were analyzed using this setup. The setup was used primarily in these studies to evaluate crater morphologies, particularly the aspect ratio of the different matrices under optimized conditions.

\subsubsection{Vis (532 $\mathrm{nm})$ LIBS In-house Setup}

The $532 \mathrm{~nm}$ in-house LIBS setup utilized a q-switched Nd:YAG dual laser head Solo PIV laser (New Wave, Fremont, CA). The output energy of the 5 ns pulse could be adjusted. Similarly to the UV in-house setup, the laser was expanded and collimated prior to focusing. The laser was focused using a $50.8 \mathrm{~mm}$ in diameter plano-convex lens, $\mathrm{f}=150 \mathrm{~mm}$. The focusing lens was also fixed on a micrometer which again yielded LTSD control. The same camera was used to observe and monitor the sample surface. The plasma emission was collected using a similar setup described above focusing the light onto the same FOC which transferred the light to the same Andor Mechelle 5000 utilizing an iCCD iStar camera. The setup was also controlled using the digital delay generator described above. The unprocessed cotton pellets were analyzed using this setup.

\subsubsection{IR (1064 nm) LIBS In-house Setup}

The in-house IR LIBS setup used a 1064 nm q-switched Nd:YAG Big Sky Ultra (Quantel USA, Big Sky, Montana) laser. The output energy of the 8 ns pulse could be adjusted. The LIBS laser setup was also, like the other in-house systems, expanded and collimated prior to focusing. The IR laser was focused using two methods: a single $50.8 \mathrm{~mm}$ in diameter plano-convex lens and also using two of the plano-convex lenses together separated with o-rings to generate a lab-built air gapped doublet. Both focusing lens setups were fixed on a micrometer stage so that LTSD could be controlled. The zoom camera lens was employed for surface monitoring. In both ablation setups the plasma light was again collected at $90^{\circ}$ to the irradiation source. The same FOC and spectrometer setup was used so that comparisons could be drawn from the different 
setups. The setup was also controlled using the digital delay generator described above. The glass and unprocessed cotton samples were analyzed using this setup.

\subsubsection{RT-100 HP Commercial LIBS Setup}

A commercial LIBS system, the RT-100 HP (Applid Spectra, Fremont, CA) was used for some analytical comparisons between commercial and in-house LIBS setups. The commercial system utilized a Nd:YAG q-switched laser operating at $1064 \mathrm{~nm}$. The maximum output energy was 50 $\mathrm{mJ}$ for the $<5 \mathrm{~ns}$ pulse. The surface of the sample was monitored using a viewing camera. The focus of this camera was linked to the laser. The laser was inherently focused into the sample to improve laser-sample interactions. The degree to which the laser was focused in to the sample could be manipulated by raising or lowering the z-stage. RT-100 HP is equipped with an Aurora six channel CCD spectrometer with a resolution of $<0.1 \mathrm{~nm}$ for UV and Vis coverage, $<0.12 \mathrm{~nm}$ for NIR coverage. The light was collected at $45^{\circ}$ to the irradiation source. The soil samples were analyzed using this setup.

\subsubsection{Determination of Laser Beam Characteristics}

The beam diameter at its smallest focal point (beam waist) was determined using a modified procedure based on the ISO 11146 (International Organization for Standardization, Geneva,

Switzerland) method for determining $\mathrm{M}^{2}$, the beam propagation factor. This factor determines the extent to which the laser beam deviates from a Gaussian beam, which would have a $\mathrm{M}^{2}=1$. Ideally using the initial beam diameter and the focal length of the focusing lens, the final, focused diameter of the beam could be calculated. This is true for Gaussian beams; however, for all other beams a correction factor, i.e., the beam propagation factor, is needed to account for deviations from the ideal. 
Beam characteristics for some of the laser beams used in the current studies were determined by maintaining the focusing lens at a constant location, while measuring the width of the beam through the beam waist ${ }^{28}$. The lasers were adjusted to the lowest power that produced the full beam profile on Zap-it laser paper (Zap-it, Salisbury, MD, USA) in order to minimize the effect of laser output on the resulting beam diameter.

\subsubsection{Determination of Mass Removal and Crater Morphology}

The mass removal for all matrices was determined by using the volume of the crater, determined by a Keyence Digital Microscope, VSX-200 (Keyence, Chicago, IL, USA). The volume was calculated on the basis of the digitally reconstructed crater, produced from a collection of combined optical slices of the crater. The mass removed in each instance was determined by comparing the density of the matrix to the volume from the microscope. The mass removal studies were focused on the cotton and glass analyses. These matrices have a density of 1.39 $\mathrm{g} / \mathrm{cm}^{3}$ and $2.5 \mathrm{~g} / \mathrm{cm}^{3}$, respectively.

The morphology of the craters for the different matrices was also monitored using the digital microscope. To compare overall crater morphology a cross-section of each crater was extracted. The cross-section was selected by scanning the crater morphology to obtain the widest, deepest part of the crater. The resulting selection appears as a 2-dimensional dissection of the crater. All matrices were compared using crater morphology and aspect ratios.

\subsubsection{Analytical LIBS Measurements}

Comparisons of the LIBS emission observed were made using optimized methods for cotton (532 $\mathrm{nm}$ and $1064 \mathrm{~nm})$ and glass (266 $\mathrm{nm}$ and $1064 \mathrm{~nm})$. To observe differences in plasma parameters and analytical conditions, methods for $532 \mathrm{~nm}$ and $1064 \mathrm{~nm}$ lasers were developed for cotton initially at low and high laser output in respect to the capabilities of the lasers. The energy values 
bracketed within the high and low outputs were determined by stepping the gate delay and the number of laser shots accumulated. The criteria listed in section 2.2.4 for determining optimal parameters were used in all analytical LIBS method development. The same parameters were used for laser head one and laser head two for the $532 \mathrm{~nm}$ laser.

The $266 \mathrm{~nm}$ and $1064 \mathrm{~nm}$ parameters for glass analysis have been previously reported ${ }^{3}$. Laser and spectrometer parameters for all cotton and glass measurements are presented in table three.

Table 3: Cotton and glass parameters for $266 \mathrm{~nm}, 532 \mathrm{~nm}$ and $1064 \mathrm{~nm}$ LIBS analysis

\begin{tabular}{|c|c|c|c|c|c|c|}
\hline Matrix & Laser & $\begin{array}{l}\text { Power density } \\
\left(\mathrm{W} / \mathrm{cm}^{2}\right)\end{array}$ & $\begin{array}{l}\text { LTSD } \\
(\mathrm{mm})\end{array}$ & $\begin{array}{c}\text { Gate Delay } \\
(\mu \mathrm{s})\end{array}$ & $\begin{array}{c}\text { Gate Width } \\
(\mu \mathrm{s})\end{array}$ & $\begin{array}{l}\text { Laser } \\
\text { Shots }\end{array}$ \\
\hline \multirow{17}{*}{ 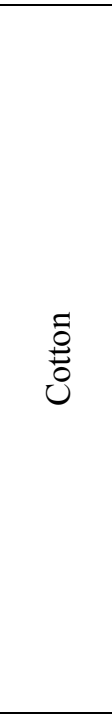 } & \multirow{9}{*}{532} & $8.08 \times 10^{9}$ & 0.9 & 1.00 & 5 & 100 \\
\hline & & $1.24 \times 10^{10}$ & 0.9 & 1.00 & 5 & 100 \\
\hline & & $1.47 \times 10^{10}$ & 0.9 & 1.00 & 5 & 100 \\
\hline & & $1.68 \times 10^{10}$ & 0.9 & 1.05 & 5 & 100 \\
\hline & & $2.10 \times 10^{10}$ & 0.9 & 1.10 & 5 & 85 \\
\hline & & $2.52 \times 10^{10}$ & 0.9 & 1.15 & 5 & 85 \\
\hline & & $2.96 \times 10^{10}$ & 0.9 & 1.20 & 5 & 70 \\
\hline & & $3.17 \times 10^{10}$ & 0.9 & 1.25 & 5 & 70 \\
\hline & & $3.30 \times 10^{10}$ & 0.9 & 1.30 & 5 & 70 \\
\hline & \multirow{8}{*}{1064} & $5.73 \times 10^{9}$ & 0.7 & 1.00 & 5 & 100 \\
\hline & & $6.96 \times 10^{9}$ & 0.7 & 1.05 & 4.5 & 100 \\
\hline & & $8.80 \times 10^{9}$ & 0.7 & 1.10 & 4.5 & 90 \\
\hline & & $1.19 \times 10^{10}$ & 0.7 & 1.15 & 4.5 & 90 \\
\hline & & $1.68 \times 10^{10}$ & 0.7 & 1.20 & 4.5 & 90 \\
\hline & & $1.94 \times 10^{10}$ & 0.7 & 1.25 & 4.5 & 90 \\
\hline & & $2.21 \times 10^{10}$ & 0.7 & 1.30 & 4.5 & 90 \\
\hline & & $2.48 \times 10^{10}$ & 0.7 & 1.35 & 4.5 & 90 \\
\hline \multirow{2}{*}{ Glass } & 266 & $1.45 \times 10^{10}$ & 1.3 & 1.20 & 3.0 & 100 \\
\hline & 1064 & $1.20 \times 10^{10}$ & 0.5 & 120 & 11.0 & 100 \\
\hline
\end{tabular}

Methods depicted in bold test correspond to the low and high energy outputs used for parameter optimization.

\subsection{Results and Data Analysis}

\subsubsection{Laser Beam Characteristics}

Laser beam properties for the $532 \mathrm{~nm}$ and $1064 \mathrm{~nm}$ setups are presented in table 4 . Because influences of laser beam characteristics are only being considered in relation to the analytical 
performance of cotton analysis for the current studies, the beam properties have only been presented for the Vis and IR lasers.

Table 4: $532 \mathrm{~nm}$ and $1064 \mathrm{~nm}$ laser beam properties

\begin{tabular}{|c|c|c|}
\hline Laser & Beam waist $(\boldsymbol{\mu m})$ & $\begin{array}{c}\text { Beam Propagation } \\
\text { Factor }\end{array}$ \\
\hline $532 \mathrm{~nm}$, Head 1 & $84.7 \pm 2.5$ & 1.57 \\
\hline $532 \mathrm{~nm}$, Head 2 & $91.6 \pm 2.8$ & 1.65 \\
\hline $\begin{array}{c}1064 \mathrm{~nm} \\
\text { (air-gapped doublet) }\end{array}$ & $72.2 \pm 2.9$ & $1.77^{1}$ \\
\hline 1064 nm (single lens) & $115.1 \pm 8.0$ & $1.77^{1}$ \\
\hline
\end{tabular}

${ }^{1}$ Actual beam propagation factor might be lower than determined. Laser beam power could not be attenuated to an energy output that resulted in no change to the Zap-it laser paper.

The differences in beam characteristics, although slight for the two $532 \mathrm{~nm}$ laser heads can be used to provide some insight into the differences, if any, in analytical performance and sensitivity that are observed for two lasers of the same wavelength that are focused onto the target under the same conditions. Although not the goal of this research, this information could aide in the evaluation of what factors are important to producing a laser beam of high analytical quality.

\subsubsection{Analytical Performance of Unprocessed Cotton Using Different Power Densities}

The analytical performance of unprocessed cotton, measured in precision (\%RSD) and SNR varied with power density and also laser irradiation wavelength. Some emission lines, representing analytes of varying concentrations are exhibited in table 5. There is a crossover point in which analytical performance, by means of SNR no longer improves. The SNR can be equated to limit of detection (LOD), and thus sensitivity. The power density at which this occurs is approximately $1.74 \times 10^{10} \mathrm{~W} / \mathrm{cm}^{2}$ and $2.52 \times 10^{10} \mathrm{~W} / \mathrm{cm}^{2}$, using the diameter of the laser beam that is in contact with the surface of the sample for $1064 \mathrm{~nm}$ and $532 \mathrm{~nm}$ LIBS, respectively. 
There is some similarity in the crossover points observed for both $532 \mathrm{~nm}$ laser heads and the $1064 \mathrm{~nm}$ lase, whic implies that it could be possible to extrapolate some operating conditions from one method $(1064 \mathrm{~nm})$ to another $(532 \mathrm{~nm})$.

Table 5: Analytical performance of $532 \mathrm{~nm}$ and $1064 \mathrm{~nm}$ LIBS for cotton analysis

\begin{tabular}{|c|c|c|c|c|c|c|c|c|c|c|}
\hline \multicolumn{11}{|c|}{1064 nm LIBS } \\
\hline Analyte & \multicolumn{2}{|c|}{$8.80 \times 10^{9}$} & \multicolumn{2}{|c|}{$1.19 \times 10^{10}$} & \multicolumn{2}{|c|}{$1.68 \times 10^{10}$} & \multicolumn{2}{|c|}{$1.74 \times 10^{10}$} & \multicolumn{2}{|c|}{$2.21 \times 10^{10}$} \\
\hline $\mathbf{B a}$ & 21.2 & 9 & 19.4 & 10 & 16.3 & 11 & 13.1 & 15 & 14.0 & 10 \\
\hline Mg & 11.7 & 650 & 10.8 & 700 & 8.8 & 720 & 7.9 & 750 & 9.9 & 700 \\
\hline $\mathbf{K}$ & 12.8 & 180 & 11.5 & 210 & 11.9 & 250 & 10.8 & 300 & 9.3 & 280 \\
\hline $\mathrm{Sr}$ & 7.2 & 21 & 5.1 & 40 & 6.5 & 55 & 4.3 & 47 & 5.8 & 48 \\
\hline \multicolumn{11}{|c|}{$532 \mathrm{~nm}$ LIBS, Head 1} \\
\hline Analyte & \multicolumn{2}{|c|}{$1.68 \times 10^{10}$} & \multicolumn{2}{|c|}{$2.10 \times 10^{10}$} & \multicolumn{2}{|c|}{$2.52 \times 10^{10}$} & \multicolumn{2}{|c|}{$2.96 \times 10^{10}$} & \multicolumn{2}{|c|}{$3.17 \times 10^{10}$} \\
\hline $\mathbf{B a}$ & 24.9 & 5 & 18.6 & 7 & 9.7 & 11 & 8.3 & 7 & 12.1 & 8 \\
\hline Mg & 17.6 & 842 & 15.1 & 898 & 9.0 & 931 & 3.9 & 964 & 12.0 & 985 \\
\hline $\mathbf{K}$ & 11.1 & 289 & 8.3 & 301 & 11.0 & 321 & 7.2 & 330 & 4.8 & 345 \\
\hline $\mathbf{S r}$ & 15.9 & 21 & 13.5 & 38 & 7.1 & 64 & 9.3 & 82 & 13.0 & 71 \\
\hline \multicolumn{11}{|c|}{$532 \mathrm{~nm}$ LIBS, Head $2^{1}$} \\
\hline Analyte & \multicolumn{2}{|c|}{$1.66 \times 10^{10}$} & \multicolumn{2}{|c|}{$2.05 \times 10^{10}$} & \multicolumn{2}{|c|}{$2.42 \times 10^{10}$} & \multicolumn{2}{|c|}{$2.87 \times 10^{10}$} & \multicolumn{2}{|c|}{$3.01 \times 10^{10}$} \\
\hline Ba & 10.5 & 6 & 8.8 & 6 & 9.3 & 7 & 7.8 & 8 & 9.1 & 10 \\
\hline Mg & 18.5 & 850 & 15.5 & 878 & 9.6 & 900 & 5.5 & 942 & 13.7 & 974 \\
\hline $\mathbf{K}$ & 6.0 & 273 & 12.5 & 290 & 3.5 & 311 & 4.7 & 324 & 7.4 & 341 \\
\hline Sr & 16.7 & 23 & 12.8 & 36 & 10.3 & 58 & 8.2 & 77 & 11.7 & 70 \\
\hline
\end{tabular}

The left of the two columns designated for each power density corresponds to precision (\%RSD), the right column, SNR. Emission lines used: Ba (II) $493.40 \mathrm{~nm}, \mathrm{Mg}$ (I) $285.21 \mathrm{~nm}$, K (I) $766.48 \mathrm{~nm}$ and Sr (II) $421.55 \mathrm{~nm}$.

Because the optimized $532 \mathrm{~nm}$ and $1064 \mathrm{~nm}$ methods utilize different LTSD this may account for some of the variation in optimal power densities. To exclude the LTSD factor from this measurement the power density for the LIBS methods were also calculated using the beam waist. The smaller beam diameter at the focal point results in a higher, but more similar power density.

The values determined for the cotton methods are $1.36 \times 10^{11}, 1.31 \times 10^{11}$ and $1.20 \times 10^{11} \mathrm{~W} / \mathrm{cm}^{2}$ for $532 \mathrm{~nm}$ head 1, $532 \mathrm{~nm}$ head 2 and $1064 \mathrm{~nm}$ LIBS analysis, respectively. The variations in LTSD influence laser-sample interactions, which differ with excitation source. To reduce the effects that pertain to a particular matrix and thus laser-sample interactions, an independent 
measurement of power density, i.e. utilizing sample surface, appears to be more applicable. However, the feasibility of this will be addressed with other matrices later in the text.

In addition, there appears to be no consistent trend in the analytical performance obtained when using $532 \mathrm{~nm}$ laser head one or laser head two, which suggests that slight differences in laser beam characteristics do not play a significant, observable role in analytical LIBS performance.

The plasma interactions should also be considered in order to determine what is producing this distinct crossover point. Electron densities were calculated using the two-line approach ${ }^{8}$. The lines selected to determine plasma conditions were the $\mathrm{Mg}$ (II) $280.27 \mathrm{~nm}$ and $\mathrm{Mg}$ (I) $285.21 \mathrm{~nm}$ emission lines. The selected emission lines had similar peak widths and the close proximity which implies similarity in detector response. In addition, $\mathrm{Mg}$ was present in all matrices considered here. To ensure that these emission lines did not suffer from self-absorption (SA), single element standards (CPI International, Santa Rosa, CA, USA) were added to the NC2005 sample to generate working response curves, figure 3 . The intensity values here were not normalized to any internal standard (IS) so that the emission process of the analyte could be detected as the concentration of $\mathrm{Mg}$ was increased.

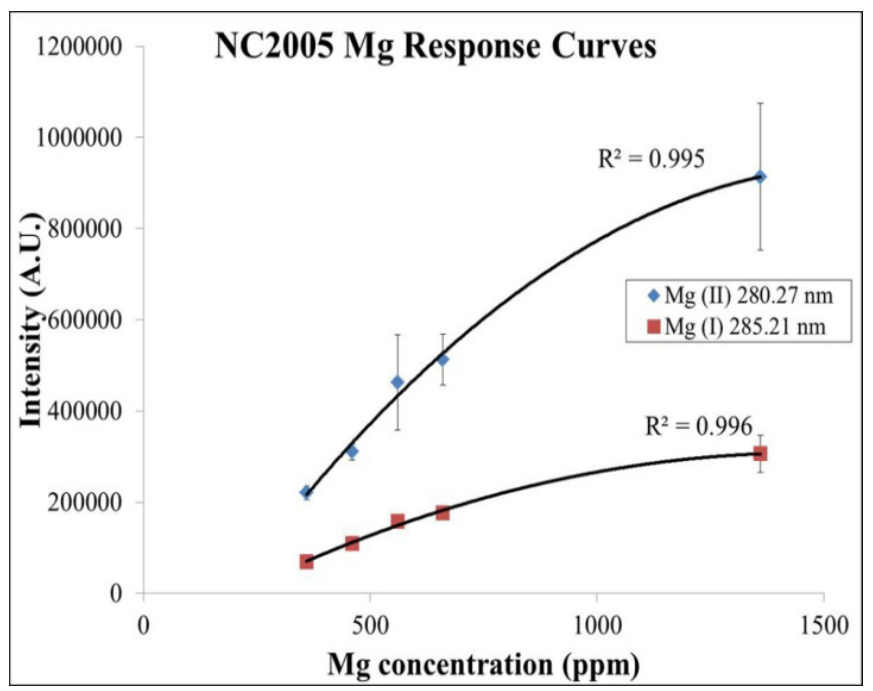

Figure 3: Mg response curves for NC2005; $\mathrm{NC2005}$ is the first data point. 
The response curves were generated at the optimum power density using the $1064 \mathrm{~nm}$ laser method. Other emission lines were considered - Sr (II) / Sr (I) and Ca (II) / Ca (I) - however, they suffered from interferences or S.A. in cotton or the other matrices considered here.

A plot of calculated electron density against the power density at sample surface, figure 4 , shows that at approximately the same point in which the precision and sensitivity shifts, the electron density does not increase in the plasma during the time in which the plasma light is collected. The leveling out of electron density is most likely the result of a change in ablation and/or plasma processes. The introduction of more energy via the excitation source produces a hotter, denser plasma that results in plasma shielding which hinders analytical measurement.

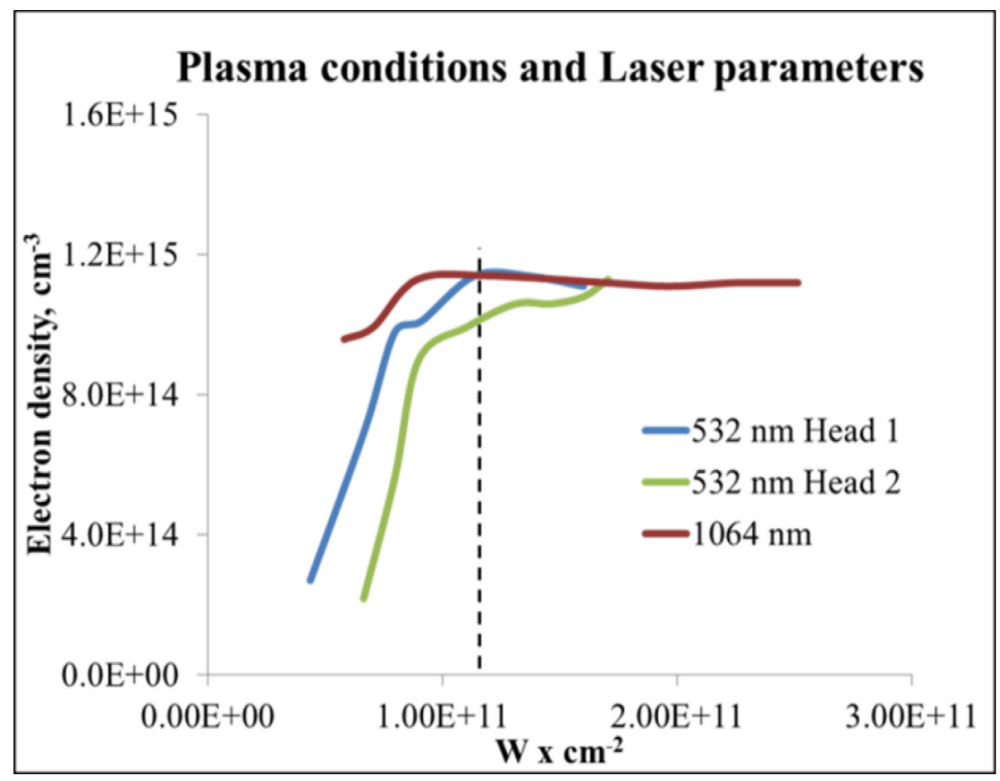

Figure 4: Electron density vs. laser power density for $532 \mathrm{~nm}$ and $1064 \mathrm{~nm}$ LIBS analysis. The dotted vertical line identifies the crossover point in analytical performance.

If an increase in electron density does occur in the plasma after the analytical crossover point, it does not contribute to the excitation and ionization of the analytes. Therefore, the electron density calculation utilized in figure 4 represents the electron density that contributes to detectable 
emission processes, rather than continuum processes. Without an increase in electron density conducive to analytical measurement the use of higher laser power density is futile and wasteful.

\subsubsection{Standardization of Laser Power Density for Different Matrices}

To determine if the concept of extrapolating a uniform power density could be applied to other matrices, glass and soil matrices were also evaluated using $1064 \mathrm{~nm}$ LIBS ablation. The power density at optimum parameters was calculated for each matrix using the $\mathrm{MgII} / \mathrm{MgI}$ ratio in order to compare to the unprocessed cotton value. To corroborate results two glass SRMs and three soil SRMs were used to obtain $\mathrm{MgII} / \mathrm{MgI}$ ratios. The differences in $\mathrm{Mg}$ concentration were used to certify that an increase in $\mathrm{Mg}$ emission intensity was observed at high concentration reducing the potential of S.A. Table 6 presents the power density at both the LTSD and at surface was also calculated for each matrix. Optimized methods for each matrix yielded a similar electron density at similar power density measurements.

Table 6: Power densities for different matrices using optimal LIBS parameters

\begin{tabular}{|c|c|c|c|c|}
\hline $\begin{array}{c}\text { Standard } \\
\text { Reference } \\
\text { Material }\end{array}$ & Mg (ppm) & $\begin{array}{l}\text { Electron } \\
\text { Density }\end{array}$ & $\begin{array}{c}\text { Power Density at } \\
\text { LTSD } \\
\left(\mathrm{W} / \mathrm{cm}^{2}\right)\end{array}$ & $\begin{array}{c}\text { Power Density } \\
\left(\mathrm{W} / \mathrm{cm}^{2}\right)\end{array}$ \\
\hline NIST 610 & 465 & $1.03 \times 10^{15}$ & \multirow{2}{*}{$1.20 \times 10^{10}$} & \multirow{2}{*}{$1.23 \times 10^{11}$} \\
\hline NIST 1831 & 21166 & $1.12 \times 10^{15}$ & & \\
\hline NIST 2704 & 12000 & $1.13 \times 10^{15}$ & \multirow{3}{*}{$1.06 \times 10^{10}$} & \multirow{3}{*}{$1.07 \times 10^{11}$} \\
\hline NIST 2710 & 8530 & $1.15 \times 10^{15}$ & & \\
\hline PACS 2 & 14700 & $1.12 \times 10^{15}$ & & \\
\hline
\end{tabular}

It should be noted that methods were developed independent of the scope of the current research and results were then evaluated for potential trends within and between matrices. Therefore, it can be assumed that the information was obtained without bias in favor of the goals of the present study. The argument that uniformity in power density can be applied to different matrices is strengthened. Furthermore, whereas the glass was analyzed using the same $1064 \mathrm{~nm}$ LIBS setup as the cotton, the soil was analyzed using a commercial LIBS system. The concept of uniformity 
of this particular laser parameter extends to other matrices, laser wavelengths $(532 \mathrm{~nm})$ and even different LIBS setups. The ability to utilize a uniform power density of $\sim 1.1 \times 10^{11} \mathrm{~W} / \mathrm{cm}^{2}$ at the surface of the target is progress towards the adoption of some important LIBS operating conditions for $532 \mathrm{~nm}$ and $1064 \mathrm{~nm}$ ablation. Simplification in LIBS parameters or instrumentation translates to less time required for method development. In addition the similarity in power density at optimal LTSD can be used to narrow the range of LTSDs considered in the LIBS optimization.

The results of the current studies presented address the applicability and existence of universal LIBS parameters and do so with explanations founded in using plasma interactions, i.e. electron density. However, to strengthen this argument, assessments on ablation processes should also be made.

\subsubsection{Cotton Crater Development to Address Standardization in Aspect Ratio}

At lower power density, a smaller crater is produced that results in less detectable emission. Although ablation and breakdown thresholds are exceeded, the analytical capabilities of the method are not sufficient to conducting trace analysis when few laser shots are used.

As the number of laser shots increase the crater depth also increases, figure five. The increase is at a much faster rate than the diameter of the crater. This has been shown in the literature for several other matrices ${ }^{29,30}$. As described earlier, the change in beam diameter becomes more subtle the further from the beam waist, which suggests that as the number of shots is increased for a laser that has been focused into the target, there becomes a point in which the beam has ablated the surface and sides of the crater so as only to result in an increase in crater depth, not diameter. 
The crater aspect ratio is a value used to numerically describe the dimensions of the crater. Since the crater increases in depth at a rate exceeding the change in diameter, a crossover point in aspect ratio, and thus ablation process should be also considered in addition to plasma conditions.

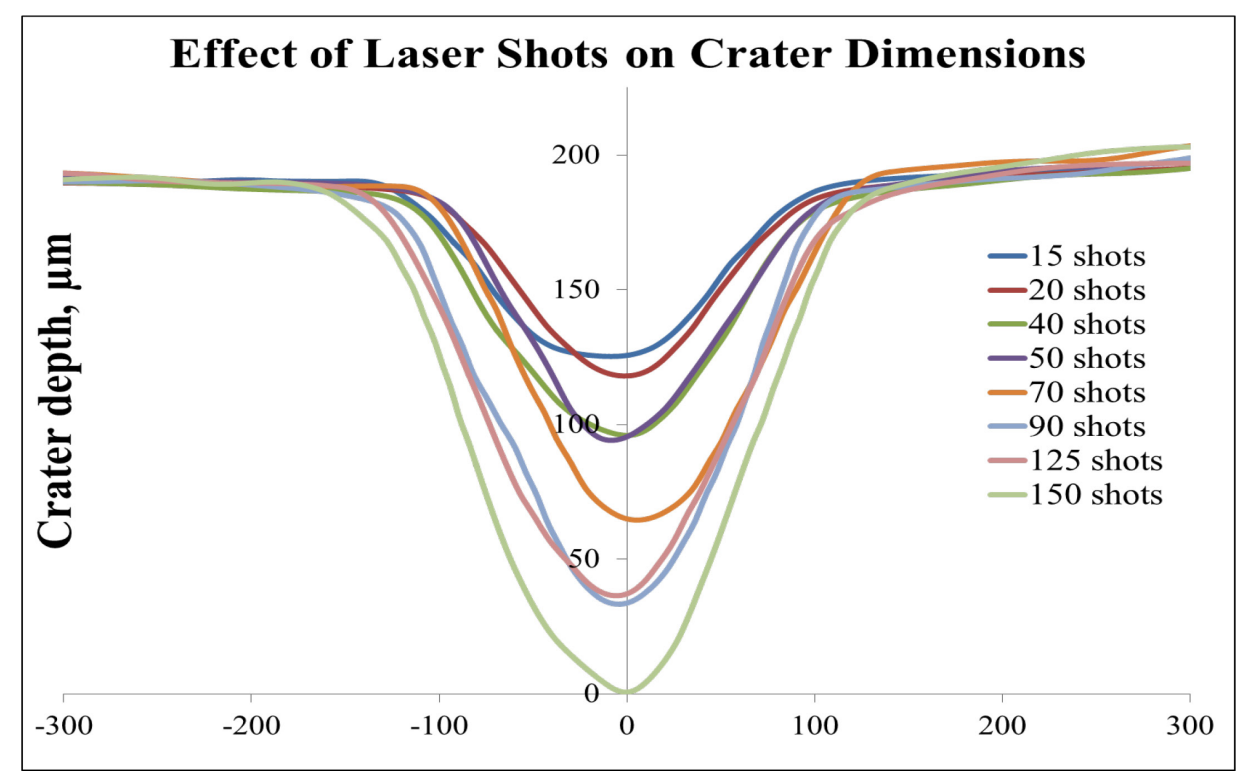

Figure 5: Resulting cotton crater as the laser shots increase. The change in crater diameter and depth as the number of repetitive laser shots in a single location is increased.

Table 7 depicts the crater aspect ratios obtained with the cotton $532 \mathrm{~nm}$ and $1064 \mathrm{~nm}$ LIBS analyses. Regardless of which $532 \mathrm{~nm}$ laser head used, the resulting crater dimensions and morphology was similar.

In addition, at its optimized number of laser shots, similar aspect ratios are observed, $0.44 \pm 0.05$ and $0.46 \pm 0.04$ for $1064 \mathrm{~nm}$ and $532 \mathrm{~nm}$, respectively. As the number of laser shots increase beyond the optimized, the change in aspect ratio decreases, suggesting that once a specific aspect ratio is surpassed analytical performance decreases for a particular matrix. Each of the cotton analytical LIBS methods utilize different LTSDs and numbers of laser shots which implies that optimization of these parameters can be corroborated by assessing the crater aspect ratio. 
Table 7: Calculated crater aspect ratios for different cotton LIBS analyses

\begin{tabular}{|c|c|c|}
\hline Matrix & \multicolumn{2}{|c|}{ Cotton } \\
\hline No. of Shots & $\begin{array}{c}1064 \mathrm{~nm}, \\
\text { air-gapped }\end{array}$ & $\begin{array}{c}532 \mathrm{~nm}, \\
\text { Head 1 }\end{array}$ \\
\hline 10 & $0.22 \pm 0.01$ & $0.13 \pm 0.03$ \\
\hline 15 & $0.22 \pm 0.01$ & $0.17 \pm 0.03$ \\
\hline 20 & $0.23 \pm 0.01$ & $0.19 \pm 0.02$ \\
\hline 40 & $0.29 \pm 0.04$ & $0.25 \pm 0.01$ \\
\hline 50 & $0.32 \pm 0.01$ & $0.30 \pm 0.02$ \\
\hline 60 & $0.35 \pm 0.03$ & $0.33 \pm 0.04$ \\
\hline 70 & $0.37 \pm 0.05$ & $0.39 \pm 0.03$ \\
\hline 85 & $0.45 \pm 0.03$ & $\mathbf{0 . 4 6} \pm \mathbf{0 . 0 4}$ \\
\hline 90 & $\mathbf{0 . 4 4} \pm \mathbf{0 . 0 5}$ & - \\
\hline 125 & $0.46 \pm 0.04$ & $0.74 \pm 0.06$ \\
\hline 150 & $0.51 \pm 0.01$ & $0.96 \pm 0.04$ \\
\hline
\end{tabular}

To determine if ablation processes can be extrapolated similarly to plasma conditions, aspect ratios were determined for another matrix. Glass crater side views generated using both the 266 $\mathrm{nm}$ and $1064 \mathrm{~nm}$ methods ${ }^{3}$, figure 6 , result in similar aspect ratios to those observed using the cotton method parameters. Optimized LIBS methods for glass by both $266 \mathrm{~nm}$ and $1064 \mathrm{~nm}$ ablation produce craters of $0.56 \pm 0.03$ and $0.48 \pm 0.06$ for SRM NIST 1831 and SRM NIST 1411 ablation, respectively. The analysis of SRM NIST 1831was conducted using $266 \mathrm{~nm}$ LIBS, while the SRM NIST 1411 crater was obtained using $1064 \mathrm{~nm}$ ablation. The glass SRMs differ in degree of transparency, which is reflected in the quality of LIBS crater generated using each technique. The transparency of SRM NIST 1831 made it difficult to produce a crater using IR irradiation. This has been reported in the literature ${ }^{3}$.

It should be noted that aspect ratios previously reported for glass were approximately $1^{3}$. The differences between the current aspect ratios are related to the lens focusing systems employed. Cylindrical and spherical lenses yield different crater dimensions which are directly related to laser focusing conditions ${ }^{31}$. Cotton using the same lens system as previously used for glass ${ }^{3}$ resulted in the aspect ratio of approximately one, demonstrated by the $3 \mathrm{D}$ crater, figure 7 . This confirms that for a single type of focusing lens, optimal parameters can also be identified using crater aspect ratios as well as presenting the applicability to several matrices. 


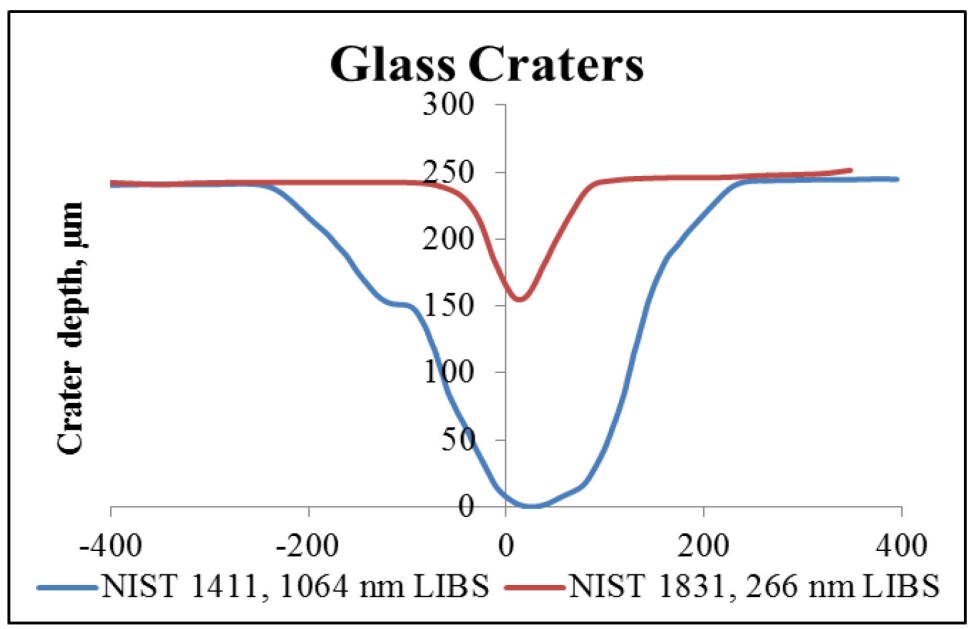

Figure 6: Glass craters obtained with $266 \mathrm{~nm}$ and $1064 \mathrm{~nm}$ LIBS.

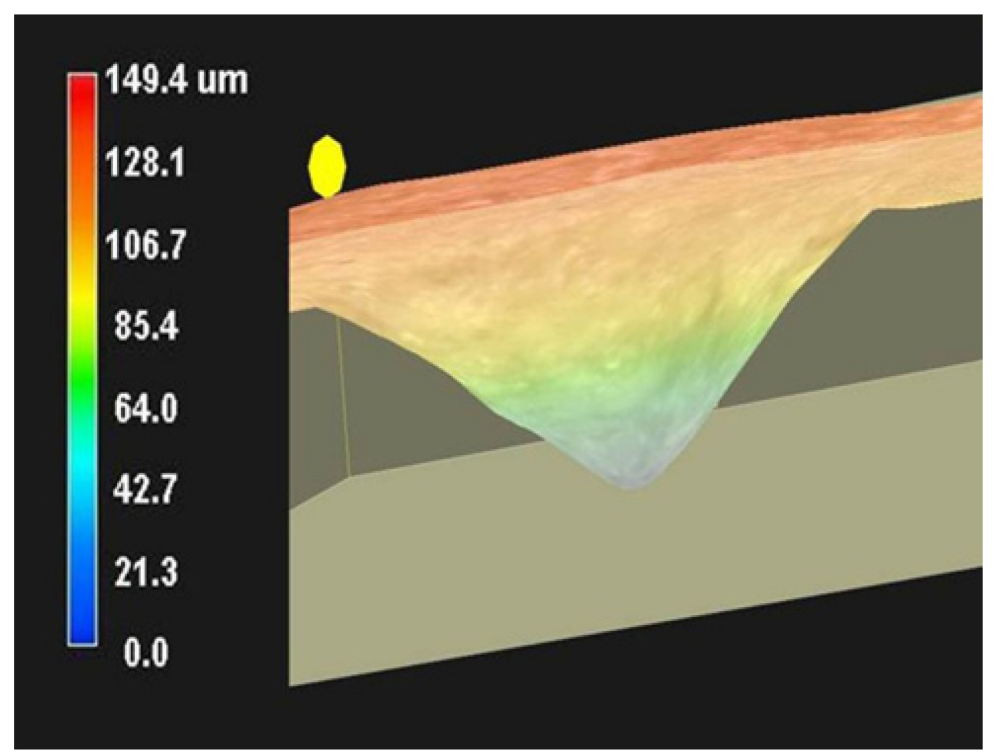

Figure 7: 3D cotton crater obtained using the same lens setup previously reported for glass analysis ${ }^{3}$.

The IR LIBS analysis of unprocessed cotton generated a $125.9 \mu \mathrm{m}$ diameter, $149.4 \mu \mathrm{m}$ depth crater.

The trend can be corroborated visually in figure eight. Significantly different crater volumes are observed for craters resulting from ablation by different irradiation wavelengths, figure 8 , for the same power density. Regardless of the focusing method used for $1064 \mathrm{~nm}$ LIBS analysis, smaller mass removal is observed in comparison to $532 \mathrm{~nm}$ ablation which always utilized a single lens focusing system. Within a particular matrix, $532 \mathrm{~nm}$ and $1064 \mathrm{~nm}$ optimized analytical LIBS 
experiments generates, although different in size, the same depth-to-diameter craters. Differences in mass removal have the potential to influence the ability to perform sensitive measurements. A comparison of the ablation efficiency in regards to analytical sensitivity is presented in the next section.

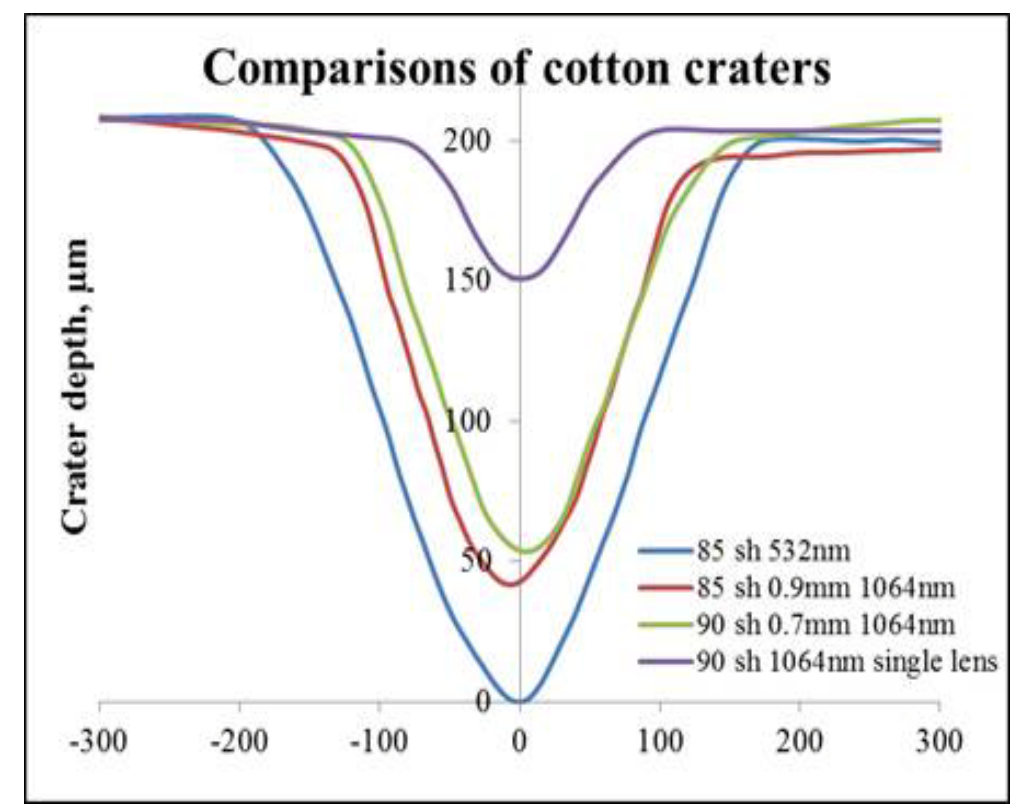

Figure 8: Cotton crater development using different laser irradiation wavelengths.

\subsubsection{Novel Approach to Relating Plasma and Ablation Processes}

To determine the influence of differences in mass removal a novel approach to assessing ablation efficiency was developed. Ablation efficiencies were evaluated in the current studies by considering both plasma conditions and ablation processes represented by a single value. A comparison was made using a ratio of intensity / mass removed (intensity $/ \mu \mathrm{g}$ ). Several cotton emission lines, table 8, were compared for different LIBS configurations.

In general, $1064 \mathrm{~nm}$ LIBS of unprocessed cotton resulted in a much higher intensity per $\mu \mathrm{g}$ of

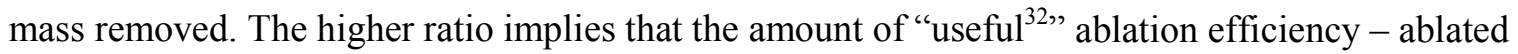
mass that contributes to the emission signal - is higher for IR than Vis irradiation at 
approximately the same power density. Additionally, IR ablation was also performed using 85 shots and a LTSD of $0.9 \mathrm{~mm}$, still yielding a higher "useful" ablation efficiency.

Table 8: Ablation efficiencies determined by $532 \mathrm{~nm}$ and $1064 \mathrm{~nm}$ LIBS analysis of cotton

\begin{tabular}{|c|c|c|c|c|c|c|c|c|c|c|}
\hline Matrix & \multicolumn{3}{|c|}{ Cotton, 1064 nm air-gapped doublet lens } & \multicolumn{5}{|c|}{ Cotton, 532 nm Head 1 } \\
\hline $\begin{array}{c}\text { No. of } \\
\text { Shots }\end{array}$ & $\begin{array}{c}\text { Mass } \\
(\mu \mathrm{g})\end{array}$ & $\mathrm{Sr}$ & $\mathrm{Mg}$ & $\mathrm{Fe}$ & $\mathrm{Ba}$ & $\begin{array}{c}\text { Mass } \\
(\mu \mathrm{g})\end{array}$ & $\mathrm{Sr}$ & $\mathrm{Mg}$ & $\mathrm{Fe}$ & $\mathrm{Ba}$ \\
\hline 10 & 3.1 & 7066 & 11782 & 2564 & 2064 & 3.1 & 1770 & 7090 & 423 & 380 \\
\hline 20 & 4.4 & 8573 & 17378 & 2961 & 3039 & 5.4 & 3420 & 7946 & 577 & 420 \\
\hline 40 & 4.7 & 14808 & 30518 & 4526 & 4787 & 9.1 & 3610 & 8189 & 578 & 402 \\
\hline 50 & 4.9 & 16630 & 34422 & 5621 & 6589 & 10.3 & 3360 & 8546 & 703 & 366 \\
\hline 60 & 5.7 & 15844 & 33361 & 5031 & 5863 & 12.1 & 3170 & 8003 & 687 & 348 \\
\hline 70 & 6.7 & 15636 & 31383 & 5133 & 5683 & 13.4 & 3030 & 8573 & 690 & 409 \\
\hline $85^{1}$ & 9.0 & 13729 & 25398 & 4408 & 4468 & 18.1 & 2308 & 7012 & 586 & 345 \\
\hline 90 & 8.4 & 16079 & 30052 & 4987 & 6709 & - & - & - & - & - \\
\hline 125 & 9.9 & 16743 & 32009 & 4745 & 7045 & 20.2 & 1540 & 6892 & 523 & 293 \\
\hline 150 & 13.0 & 15047 & 26296 & 4176 & 5440 & 21.2 & 1580 & 6173 & 487 & 222 \\
\hline
\end{tabular}

Emissions lines used: Ba (II) $493.40 \mathrm{~nm}$, Fe (I) $428.24 \mathrm{~nm}, \mathrm{Mg}$ (I) $285.21 \mathrm{~nm}$ and $\mathrm{Sr}$ (II) $421.55 \mathrm{~nm}$

${ }^{1}$ Used 532 nm cotton method parameters.

As demonstrated in table 8 , there is a range of laser shots for which the intensity/ $\mu \mathrm{g}$ ratio is consistent, which can also be used to imply that the amount of mass being converted to emission processes is also consistent. Therefore, "useful" ablation efficiency is consistent for a range. Within the range, the crossover points in analytical performance, plasma conditions and crater dimensions occur as well. This consistency can be used to draw conclusions regarding the material being ablated; ablation of a homogenous matrix will likely result in a plasma of reproducible emission processes.

The ablation efficiency begins to drop off as the number of laser shots exceeds the optimized parameters, for each LIBS configuration. The uniformity in intensity $/ \mu \mathrm{g}$ changes when the optimum crater aspect ratio is surpassed. The similarity of the calculated ablation efficiency prior to the drop-off also implies consistency in the laser-sample interactions for repetitive laser shots at the same location. The quality of laser-sample interactions is also important to developing a reproducible method. 
The studies presented here suggest that parallels can be drawn between the plasma and ablation processes when developing LIBS methods, especially as demonstrated by $532 \mathrm{~nm}$ and $1064 \mathrm{~nm}$ analytical LIBS.

\subsection{Discussion and Conclusions}

The results of the current studies demonstrate that there are LIBS operating conditions which can be adopted for a single matrix, regardless of LIBS setup, for $532 \mathrm{~nm}$ and $1064 \mathrm{~nm}$ ablation. In addition, these parameters can be extrapolated to other matrices. The uniformity in power density, $1.1 \times 10^{11} \mathrm{~W} / \mathrm{cm}^{2}$ at sample surface, for several matrices proposes the adoption of universal LIBS parameters. An optimal crater aspect ratio, $\sim 0.5$, was found to be independent of matrix for the particular LIBS focusing lens system employed in the current studies. The studies presented here provide an additional tool to evaluate LIBS parameters during method development. Evaluation of the conditions has been two-fold: using plasma processes, by means of electron density and also by considering the performance of the ablation. A balance between these two factors yields a more effective analytical LIBS method.

In addition, this work also yielded a novel method for assessing the ablated material that contributes to detectable emission. This approach combines emission processes and mass removal into one value, allowing a single assessment to be used to monitor influences of processes related to both the plasma composition and the performance of the ablation. A single ratio could be used in order to gain insight into these complex mechanisms and simplify interpretation.

Similarity in results for power density and crater dimensions for different matrices that possess different physical properties and responses to ablation strengthen the argument for universal parameters. The results of the current studies suggests that this concept extends past cotton, glass 
and soil analysis to other matrices that utilize repetitive laser shots in the same location to perform LIBS analysis by generating a crater.

\subsection{AN APPROACH FOR MEDIATING MATRIX EFFECTS IN ICP-OES}

\subsection{Overview}

The use of ICP-OES for several elemental analysis applications has been demonstrated throughout the scientific literature and textbooks ${ }^{5,33}$. The solution sample introduction utilized in this technique requires the digestion of solid matrices or the adjustment of the composition or $\mathrm{pH}$ of samples already in solution by means of adding an acid. While the matrix may appear to no longer be a factor, the presence of organic and inorganic constituents in the sample can still have an impact on the analytical data obtained. One commonly encountered interferent in ICP-OES involves the influence of matrix effects; the most prominent and crucial of these relating to suppression or enhancement effects that occur in the plasma. The presence of matrix effects can result in the under or over accounting of analytes present, leading to a bias in results. Suppression or enhancement, in most cases, is the result of changes in the plasma conditions that have an effect on the excitation and ionization mechanisms in the plasma, and therefore influence the intensity of the subsequent emission processes. The focus of this chapter is to explore the ability to buffer the aerosol that is being introduced in to the ICP torch so as to minimize or regulate possible matrix effects, reducing or even eliminating bias in analytical results.

\subsection{Introduction}

\subsubsection{Plasma Interactions in ICP-OES}

The ICP-OES technique employs a plasma torch at atmospheric pressure in argon (Ar) for sample atomization, excitation and ionization ${ }^{34}$. The technique is classified as an atomic emission 
spectrometric tool, so ICP-OES and ICP-AES (inductively coupled plasma atomic emission spectrometry) can be used interchangeably.

There are several mechanisms that take place simultaneously in the plasma, making it a very complex environment. Excitation and ionization can involve several pathways, some of which have been described in section 2.2.1. A common excitation mechanism is known as Penning ionization. It involves a reaction between an excited gas molecule and a neutral atom ${ }^{5}$. The interaction results in the excitation of the atom which leaves behind a neutral gas atom. Because the ICP consists of an Ar environment that requires constant excitation of the Ar gas to maintain the plasma, the opportunity for Penning ionization to occur is extremely high. In general, Penning ionization refers to the reaction between an excited state gas molecule with a neutral atom ${ }^{5}$. In an Ar ICP the Penning ionization mainly takes place with the argon-metastable atoms which have excitation energies of 11.55 and $11.76 \mathrm{eV}^{35,36}$. Generic reactions with the metastable states can be found in equations 7 and 8 where $\mathrm{Ar}^{\mathrm{m}}$ corresponds to an argon metastable atom, and $=\mathrm{X}^{+}$and $\mathrm{X}^{+*}$ correspond to ion and excite state species, respectively.

$$
\begin{gathered}
A r^{m}+X \rightarrow A r+X^{+} \\
A r^{m}+X \rightarrow A r+X^{+^{*}}+e^{-}
\end{gathered}
$$

$\mathrm{The}^{\mathrm{m}}{ }^{\mathrm{m}}$ atoms can only interact with atoms for which the IP and excitation energy together is less than the excitation energy of the metastable state(s). In addition subsequent interactions based on charge transfer can take place with argon.

$$
\begin{gathered}
A r^{m}+e^{-} \rightarrow A r^{+}+2 e^{-} \\
A r^{+}+X \rightarrow A r+X^{+}
\end{gathered}
$$




$$
A r^{+}+X \rightarrow A r+X^{+^{*}}
$$

For charge transfer with Ar to take place, the IP and excitation energy cannot exceed that of Ar, $15.76 \mathrm{eV}$ or the energy of the metastable states. While both Penning ionization and charge transfer mechanisms can take place for any species below the specified energy, these mechanisms have been shown to favor species when ionization plus excitation potential is close to the $\operatorname{Ar}^{\mathrm{m}}$ and Ar gas species ${ }^{5}$. There are four possible metastable species that vary in energy and lifetime. Two of which have very long lifetimes, as a result of the single-photon transitions to ground state being forbidden ${ }^{37}$. A major difference between metastable species and excited Ar species is that the temporal and spatial distributions of them vary as the amount of sample introduced increases and also over the duration in which the sample is being introduced ${ }^{36}$.

Other excitation mechanisms are the result of a form of collision. Collisions that can contribute to excitation and ionization have been listed in section 2.2.1. One form of collisional excitation is referred to as a charge transfer mechanism ${ }^{38-40}$. Similarly to what is described above in equations 9-11, this occurs when an ion $\left(\mathrm{X}^{+}\right)$transfers energy to another species $(\mathrm{Y})$ in the plasma. For this to occur, the IPs of the two interacting species must be very similar, implying the interaction could occur in either direction.

$$
\mathrm{X}^{+}+\mathrm{Y} \leftrightarrow \mathrm{Y}^{+}+\mathrm{X}
$$

Another, and probably the most abundant type of collision involves an electron. These collisions can be generically described as collisional excitation of a target atom (X) with an electron to yield an ion $\left(\mathrm{X}^{+}\right)$of target:

$$
\mathrm{X}+\mathrm{e}^{-} \leftrightarrow \mathrm{X}^{+}+\mathrm{e}
$$


The mechanisum can also take place to result in excitation to an ion $\left(\mathrm{X}^{+}\right)$to yield an excited state $\left(\mathrm{X}^{+\bullet}\right)$

$$
\mathrm{X}^{+}+\mathrm{e}^{-} \leftrightarrow \mathrm{e}+\mathrm{X}^{+\cdot}
$$

Electrons are the most prominent species of a plasma because they are a product of the constantly reoccurring excitation of $\mathrm{Ar}$ gas and the Penning ionization, the dense population of electrons makes these forms of collisional excitation very important. Because of this, the electron density, which is a measurement of the amount of electrons present in the plasma, is then an indicative parameter pertaining to plasma diagnostics.

The electron density can be calculated by considering several different relationships in the plasma. The Saha and Boltzmann equations have been described in section 2.2.3. Both of these equations can be used to describe processes in atomic emission techniques, including LIBS and ICP-OES analysis.

Not including charge transfer, ionization and excitation can occur from either excited gas molecules of electrons. While both of these mechanisms are able to excite almost all elements on the periodic table, the ease in which this can occur is a function of the IP of the element. The IP, which has been defined in section 2.2.1, is definite for each element. In addition each element has more than one IP; although it often requires such a significant amount of energy to obtain these ions that only the first and second IPs are of any importance. In atomic emission techniques like ICP-OES, the IP defines the ease in which the element is ionized and can determine potential influences the analyte may have in the $\mathrm{ICP}^{41}$. The lower the IP, the more readily ionized the element. Some elements have a much lower first IP than others and can influence the possibility of other, higher IP elements from being ionized. This type of element is referred to as an easily ionizable element (EIE) and includes the first group of the periodic table. These elements have an 
extremely low first IP $(\sim 4 \mathrm{eV})$ in comparison to other elements $(\sim 7 \mathrm{eV})$. In addition, EIEs have such a high second IP, that only atomic species for these elements exist in the plasma. The presence of these elements in the matrix is reflected in the ionization equilibrium (ionic species/atomic species ${ }^{38}$ ), resulting in a shift towards the atomic species. The shift affects the sensitivity in detecting other, higher potential elements, both atomic and ionic. The presence of EIEs in a matrix is a source of interferences that directly impact the ability to accurately detect and quantify analytes in ICP-OES. While some research has suggested that buffering of samples with EIEs like cesium $(\mathrm{Cs})$ or sodium $(\mathrm{Na})$ can diminish the effect by flooding the plasma with these elements in all samples ${ }^{42-44}$, contradictory results exist ${ }^{45-48}$. Also, introducing additional constituents reduces the overall rate of mass transport, decreasing the amount of analyte reaching the plasma which is then available for detection ${ }^{49}$. The less efficient mass transportation is magnified by the fact that the EIEs still cause an ionization shift, regardless of their so-called buffering capabilities.

Other specific element groups can also interfere with the excitation and ionization of other analytes. The elements of this type include those with low second IPs and rare earth elements (REEs). Elements that have a low second IP include $\mathrm{Ba}, \mathrm{Ca}$, and $\mathrm{Sr}$. The presence of these elements can hinder the detection other emission processes due to their ability to quench the Ar excited states ${ }^{46}$.

Regardless of whether or not the elements are the focus of detection the mere presence can influence the quality of measurement being made if present in the samples at varying concentrations. While the literature addresses and attempts to explain this type of interferent $^{41,46,47,49-52}$ more of an effort to reduce the bias in analytical analysis is necessary. It is because of this that more effective approaches to buffering or reducing shifts in ionization equilibrium should be explored. The focus of this chapter considers the use of noneasily ionizable 
elements (NEIEs) to buffer the solution from the opposite manner as employed in the use of EIEs. In addition this chapter explores a novel method of mediating plasma interactions to minimize encountered biases.

\subsection{Materials and Methods}

\subsubsection{Description of Samples and Sample Preparation}

\subsubsection{Matrix Solution Preparation}

Matrix solutions were classified as any single element, or combination of elements present in high concentration $(0.01 \%$ or higher), making them a more prominent component of the bulk solution being analyzed. Regardless if selected for analysis or monitored, the presence of these elements can influence plasma dynamics.

Matrix solutions were made in large batches of $100 \mathrm{~mL}$ so that the same solutions could be used is several studies for comparison purposes. This is a large volume when considering that a single ICP-OES analysis requires $\sim 3 \mathrm{~mL}$ of sample.

All elemental matrix constituents were of certified ICP grade purity (CPI, RICCA) and were diluted to the working concentration using 0.8M nitric acid, optima grade (Thermo Fisher Scientific, USA). Table 9 lists several matrix constituents explored in this research and reasoning for selection. 
Table 9: ICP-OES matrix constituents

\begin{tabular}{|c|c|}
\hline Matrix Constituent & Rationale for Including \\
\hline Potassium $(\mathrm{K})$ & EIE, common matrix element \\
\hline Cesium (Cs) & EIE, reportedly used as a buffer \\
\hline Sodium $(\mathrm{Na})$ & $\begin{array}{l}\text { EIE, common matrix element, } \\
\text { reportedly used as a buffer }\end{array}$ \\
\hline Lithium (Li) & EIE (confirm effects of other EIEs) \\
\hline Scandium $(\mathrm{Sc})$ & $\begin{array}{c}\text { REE, often used as an IS, determine } \\
\text { extent of effects }\end{array}$ \\
\hline Yttrium (Y) & $\begin{array}{c}\text { REE, often used as an IS, determine } \\
\text { extent of effects }\end{array}$ \\
\hline Lanthanum (La) & REE \\
\hline Samarium $(\mathrm{Sm})$ & REE \\
\hline Calcium $(\mathrm{Ca})$ & Low $2^{\text {nd }} I P$, common matrix element \\
\hline Zinc $(\mathrm{Zn})$ & High 1 $^{\text {st }}$ IP \\
\hline Cadmium $(\mathrm{Cd})$ & $\begin{array}{c}\text { High } 1^{\text {st }} \text { IP, used to corroborate } \mathrm{Zn} \\
\text { effects }\end{array}$ \\
\hline
\end{tabular}

The elements listed in table 9 are not all-inclusive, but span important classes or types of possible interferences. The prominence of these interferences across different types of sample sources stresses the practical implications to controlling plasma interactions.

\subsubsection{Analyte Solution Preparation:}

All analyte solutions were prepared in much the same manner as the matrix solutions with one exception. Analyte solutions were prepared fresh with every study conducted.

Analytes were first considered based on the value of the first and second IP of the element. The IP influences which plasma interactions are possible and the extent to which they take place in the plasma. The initial classification of the analytes can be broken down in to five groups:

1) $1^{\text {st }} \mathrm{IP}<5.4 \mathrm{eV}$ (displayed in green in all data related figures)

2) $5.4 \mathrm{eV}<1^{\text {st }} \mathrm{IP}<9.0 \mathrm{eV}, 2^{\text {nd }} \mathrm{IP}<11 \mathrm{eV}$ (displayed in blue in all data related figures)

3) $5.4 \mathrm{eV}<1^{\text {st }} \mathrm{IP}, 11 \mathrm{eV}<2^{\text {nd }} \mathrm{IP}<15.75 \mathrm{eV}$ (displayed in red in all data related figures)

4) $5.4 \mathrm{eV}<1^{\text {st }} \mathrm{IP}<9.0 \mathrm{eV}, 15.75 \mathrm{eV}<2^{\text {nd }} \mathrm{IP}$ (displayed in orange in all data related figures)

5) $9.0 \mathrm{eV}<1^{\text {st }} \mathrm{IP}$ (displayed in yellow in all data related figures) 
Analytes were selected from each of the IP ranges above in order to determine what trends and generalizations could be made based on this property.

Additionally, other elements were selected because they could be used to probe properties of the plasma. These properties are important in the mechanisms leading up to emission and have been described in elsewhere in the text (section 2.2.1). Analyte and analyte emission lines were also selected on the basis of previously outlined criteria (section 2.2.4). To ensure that the analytes in these IP ranges performed similarly from day-to-day so as to make comparisons across different studies, analytes of different IP values were analyzed daily. Figure 9 presents an example of the variation encountered.

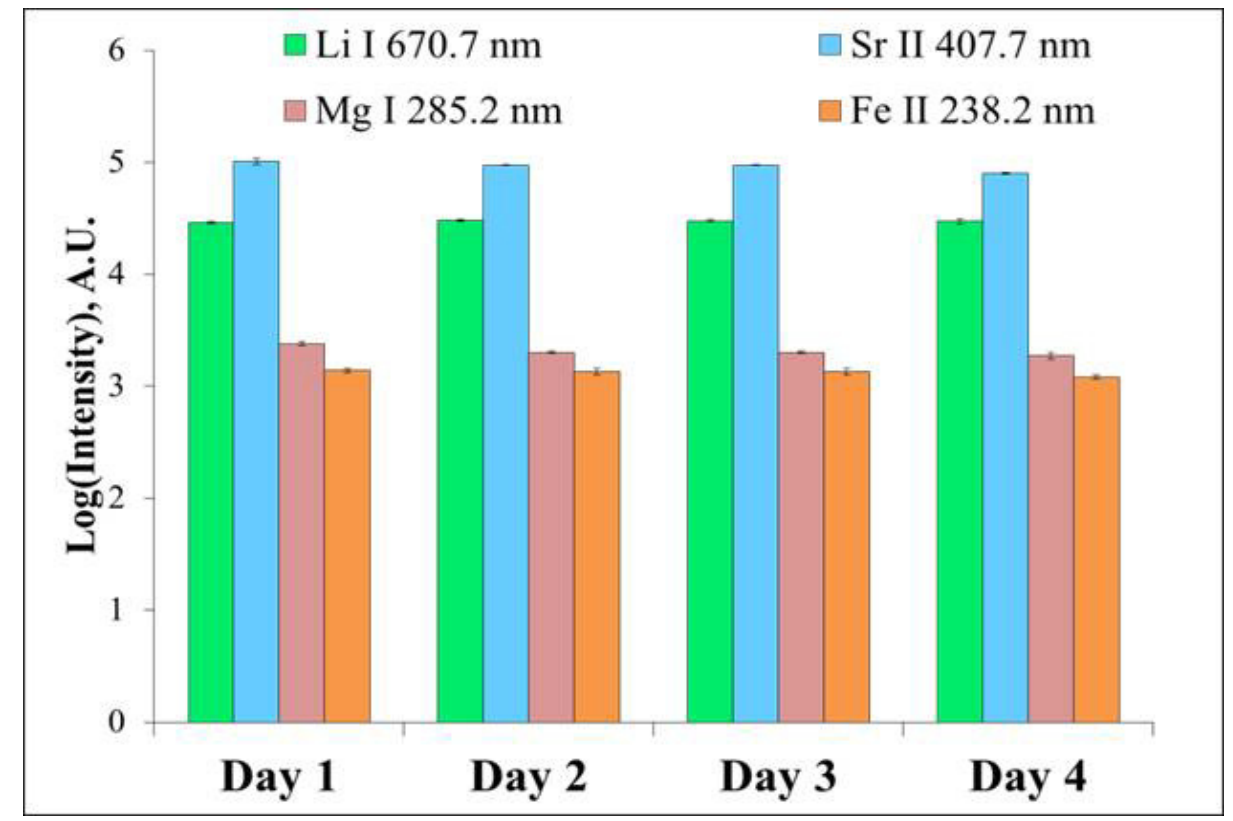

Figure 9: Day-to-day variation in ICP-OES response based on analyte IP.

\subsubsection{Principles of ICP-OES}

Although ICP-OES and LIBS are atomic emission tools, several aspects of these techniques differ. For example, the plasma generated in each respective approach differs in size and lifetime. The plasma that is produced in ICP-OES is a continuous, sustained plasma whereas a LIBS 
plasma, as previously described, results in a transient, short-lived plasma. Both techniques, however, result in the detection of emission processes. Emission takes place following ionization and excitation on an atomic level in the plasma. These mechanisms have been described in more detail in both 2.2.1 and 3.2.1.

A typical ICP-OES setup consists of a 1) sample introduction mechanism 2) torch and 3) an optical spectrometer. For the sake of this research the means of introducing the sample/analyte is by laser ablation, a solid sampling technique, further described in section 4.3.2.1. The torch used in ICP analysis is made of quartz with several channels for Ar flow to cool the plasma (0.5-1.0 $\mathrm{L} / \mathrm{min})$, to direct the analyte through the central path $(0.4-0.8 \mathrm{~L} / \mathrm{min})$, and also to provide high volumes of Ar to feed the plasma $(15-20 \mathrm{~L} / \mathrm{min})$. The flows of gas for these purposes correspond to the outer, intermediate and inner or central channels of the torch, respectively. The torch can be a single quartz piece, or, in the instance of the research conducted within this dissertation, the outer and intermediate channels are composed of quartz, while in the innermost channel also consists of an alumina injector that is able to resist corrosion when high concentrations of acids are aspirated.

The torch rests within a multi-loop copper load coil that receives power from a radio frequency (RF) generator that is then used to sustain the plasma. A current within the load coil oscillates (27 or $40 \mathrm{MHz}$ ) as the RF power (700-1500 watts) is applied. The purpose of the RF oscillation is to generate electric and magnetic fields at the end of the torch. The combination of this field, argon gas and a spark, results in the stripping of an electron from argon gas atoms. The electrons are accelerated by the field which adds energy ${ }^{4}$. These accelerated, high-energy electrons go through a series of collisions with other argon atoms, stripping more electrons. The repetitive action results in the generation of a plasma gas that consists of Ar atoms and ions, and electrons ${ }^{4}$. The plasma will persist as long as there is gas and energy to drive the process. 
Several advancements in the instrumental design of ICP-OES has renewed interest in this technique for solution and solid sampling. In addition to a brief introduction to the manner in which the emission is detected, a description of some of these advancements will be presented in order to emphasize the increase in instrument flexibility.

The light from the emission processes in the plasma is polychromatic and upon collection, needs further separation so the individual species can be determined. An optical spectrometer consists of several components which collect, resolve and detect the radiation of individual, characteristic wavelengths. Optical windows are used to collect and transfer the emission light to the spectrometer where a dispersive device separates this beam of light into wavelengths. The most common manner of light separation is through the employment of a grating. Gratings use the principle of diffraction to separate and direct the light along different, identifying paths to the detector. As the light comes in contact with the gratings or grooves it is diffracted. The degree of diffraction depends on the particular wavelength of light. The ability to resolve narrow wavelengths depends on the number of gratings or grooves present in the dispersive component. The more grooves per millimeter, the higher the resolving power of the spectrometer. The light is collimated prior to being exposed to the grating to ensure that the separation is consistent, predictable and reproducible.

The wavelengths of light are then directed to the detector which measures the intensity of the light by application of a photo multiplier tube (PMT) or a charge transfer device (CTD). A PMT amplifies the signal associated with the light so that low levels of emission can be detected ${ }^{53}$. In addition it can handle strong signals, which expands the working dynamic range of this detector. On the other hand, charge coupled devices (CCDs) are the most common of the CTDs to be installed in ICP-OES instruments ${ }^{4,5}$. A CTD uses silicon dioxide on a silicon wafer substrate to absorb the wavelengths of light. The substrate is read in a grid-like manner with wavelengths 
being directed to specific region or pixels of the wafer. The actual detection of light and attributing it to an intensity or amount is determined on the basis of the electrons captured at the interface of the silicon dioxide and substrate ${ }^{4,54}$. The amount of light is proportional to the number of electron at accumulated at the interface. The light from the emission processes was collected using the conjunction of two read parameters: the integration and readout times, reported in seconds. The integration time is the duration in which light is collected, while the read time is the time over which the signal collected during the integration is averaged.

There have been several developments in the ICP-OES technique. Because ICP-OES is a continuous plasma many processes are happening simultaneously (emission, self-absorption, continuum radiation etc.) several advancements in the instrumental design of ICP-OES have focused on emission light collection and detection within the optical spectrometer. The improvements have enhances the analytical capabilities resulting in a more versatile technique. For example, the emission processes in the plasma can be viewed from two directions (dual view), yielding an extremely broad dynamic range and improved sensitivity. This and other design advancements also aide in overcoming other undesirable plasma interactions that can interfere with the emission process.

Newer instruments are capable of viewing the normal analytical zone (NAZ) plasma across that central channel and over the entire length (axial) or as a narrow vertical slit along the central channel (radial) while also manipulating viewing height and location in the analytical zone ${ }^{55}$. 


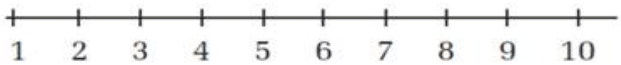

Orders of Magnitude of Signal Intensity

Figure 10a: Typical linear range achieved for different ICP-OES plasma views ${ }^{56}$.

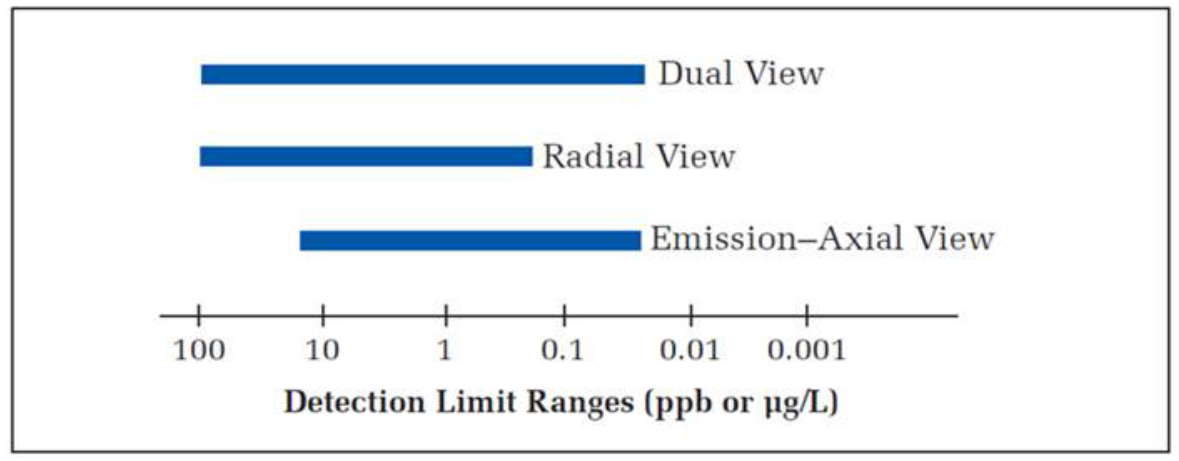

Figure 10b: Sensitivity ranges for different views of the ICP-OES plasma ${ }^{56}$.

Axially viewing provides the most sensitive view of the emission processes taking place because it collects light down the entire channel of the analytical zone; however it also is prone to saturation in the detection of this light because of the abundance. Radial viewing provides a shifted range in light collection able to collect analytes that would saturate the detector when viewed axially. In addition other instrumental design innovations remove interferences like selfabsorption, described previously, that occurs in the cooler, outer regions of the plasma. The use of a shear gas does literally that - shears off the cooler portions of the plasma eliminating this common interferent. While the main concern is indeed analytical analysis, the flexibility in light collection also provides an investigative tool to plasma composition and insight into the location of emission processes. 
In addition to improvements in plasma viewing conditions there have also been significant advancements in the capabilities of the spectrometers used for emission light collection. Newer ICP-OES instruments utilize two dispersive components to separate the light in place of a single device. The two dispersive methods that are combined include an echelle grating and a cross disperser. The use of two dispersers over one has several advantages. Echelle gratings are capable of high light efficiency spanning the wavelength range encountered in OES (160-450 nm) while diffraction based gratings have been optimized using a single wavelength (blaze wavelength) ${ }^{4}$. In addition a cross disperser improves the resolution ability of the spectrometer.

Detection of light intensity which has been typically accomplished using PMTs and CTDs like a charge coupled device (CCD) has been replaced by a newly developed segment charge coupled device (SCD). A SCD has built in dark channels on the camera board so as to minimize the effect of saturation of the pixels, which would then bloom into other pixels that correspond to other emission lines, swamping out the signal ${ }^{4}$. An SCD minimizes detection related interferences. However, there is a drawback to this approach in that there is limited space on the camera chip, which reduces the number and variety of emission lines, which can be detected. Because of this, each element may have as few as one and as many as six emission lines possible for selection. These emission processes are in the form of narrow wavelengths of light that are characteristic of particular elements on the periodic table. For most current instruments this translates to $\sim 73$ elements. The value of these advancements has been explored within the scope of this research. The advantages of the instrumentation described above have renewed interest in this analytical approach. The instrumental improvements, in addition to the fact that ICP-OES does not require cumbersome, expensive vacuum pumps like ICP-MS are leading to an increase in popularity. However, there are also disadvantages of the technique. Solution analysis still requires extensive sample preparation involving harsh acids. In addition solution analysis also generates a 
significant amount of liquid waste as a result of the constant introduction of sample, acid solution to clean the sample introduction system or acid rinse solution to cleanse the autosampler between sample injections.

\subsubsection{ICP-OES Experimental Design}

\subsubsection{ICP-OES Setup and Instrumentation}

A PerkinElmer 7300DV ICP-OES was used to conduct all experiments discussed in this chapter. The setup utilized a Scott spray chamber, equipped with a cross-flow gem tip nebulizer. The nebulizer is of the pneumatic type, designed to output high gas flows perpendicular to the sample inlet to aide in the breakup of the liquid stream into a fine aerosol ${ }^{57}$. The sample stream is restricted prior to coming in contact with the gas by means of a sapphire capillary tip. The spray chamber is then used to transport the smallest of particles $(10 \mu \mathrm{m} \text { or smaller })^{5}$ to the injector and subsequently the ICP torch. The setup is particularly advantageous for the experiments conducted within, mostly because it is resistant to clogging, which could be pertinent when high concentration matrix constituents or salts are aspirated.

The most important of the instrumental setup was the sample/matrix mixing system that was inline with the sample capillary. Figure 11 presents a two line system for separate yet simultaneous sample and matrix element introduction. One line was dedicated to the analyte (line one) while the other to the matrix or interferent (line two) in question. When a matrix was not being introduced through line two, $0.8 \mathrm{M}$ nitric acid was continually pumped through this line. Since the matrix elements were prepared in the same $0.8 \mathrm{M}$ nitric acid the purpose of flushing this line was two-fold: 1) the matrix element and $0.8 \mathrm{M}$ nitric acid solution have approximately the same density and thus maintain almost equivalent back pressure for both the analyte sample line and the spray chamber setup as well as 2) the constant introduction of a second solution eliminates 
variations in results that arise in part from differences in transport efficiency of the analyte. The pump operated at $1.5 \mathrm{~mL} / \mathrm{min}$ solution uptake for both introduction lines. The analyte results could be falsely elevated if no solution was pumped through the second line; a higher mass of analyte would reach the plasma, increasing the intensity of emission process.

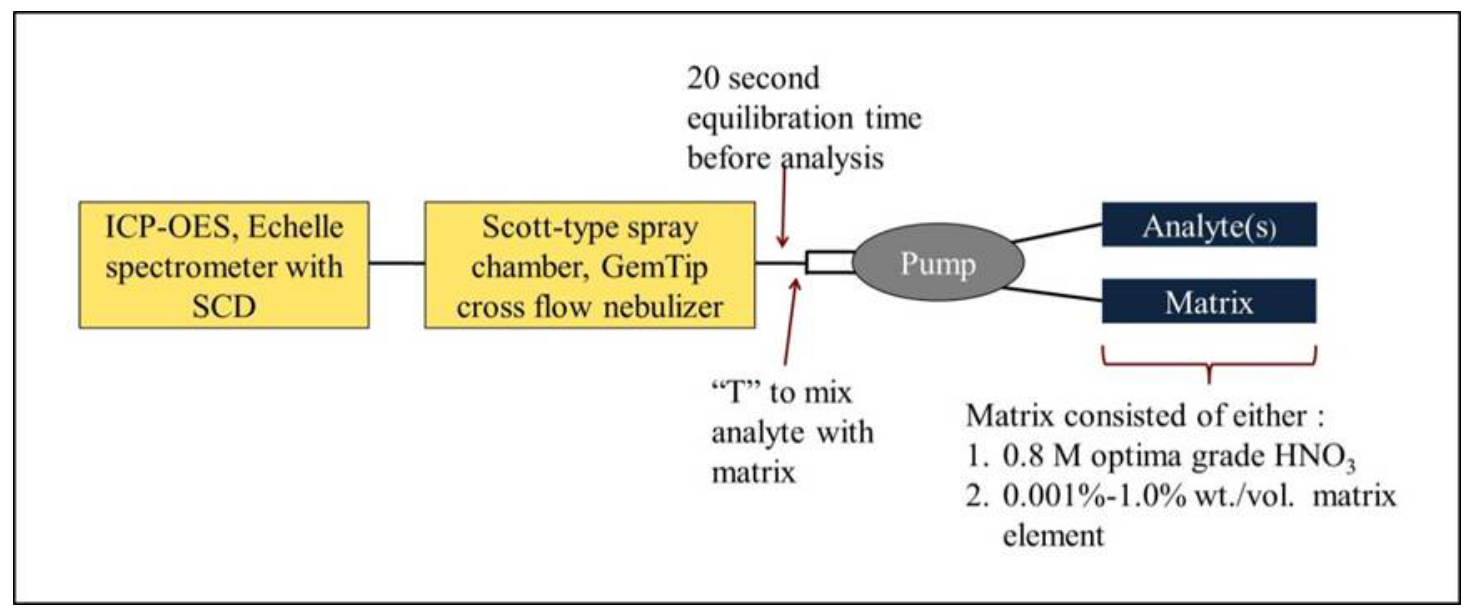

Figure 11: Schematic of ICP-OES sample introduction system.

The two sample lines were combined using a "T" type connection (Thermo Scientific, Rochester, NY, USA). Additional tubing was placed in-line between the " $T$ " and the spray chamber assembly so that a $20 \mathrm{~s}$ equilibration could be established and mixing could take place.

All studies have been conducted in the axial, or end-on, plasma viewing mode to assess the practicality of these studies for trace analysis. The RF power, $1300 \mathrm{~W}$, was held constant for all studies. The plasma gas composition was exclusively Ar, consisting of $15 \mathrm{~L} / \mathrm{min}, 0.5 \mathrm{~L} / \mathrm{min}$ and $0.7 \mathrm{~L} / \mathrm{min}$ for the plasma, auxiliary and nebulizer flows, respectively. A measurement was taken using 5 replicate readings, using uniform $1 \mathrm{~s}$ integration and read time parameters for each analyte. The concentrations of the analytes were such that integration and read parameters were not as important as in other studies conducted later in this dissertation. The steady introduction of the aspirated solution allowed for this. 


\subsubsection{Criteria for Evaluation of Studies}

Studies were evaluated from two different perspectives in order to obtain a more inclusive picture regarding the influence of the matrix constituents. The two methods pertained to the 1) conditions of the plasma and 2) effect on the analytical capabilities of the technique.

Changes in plasma conditions were evaluated using ionic/atomic emission line ratios, which are correlated to plasma temperature and the electron density. The changes were compared to changes observed in the relative intensity of the analyte response. Relative intensity can be described by the following:

$$
I_{\text {Relative }}=\frac{I_{\text {Case 2 }}}{I_{\text {Case } 1}}
$$

where I in all accounts refers to measurements of intensity. The intensity obtained in case 1 corresponds to normal operating conditions, while case 2 represents a change in the operating parameters. A relative intensity of one therefore implies no effect on emission regardless of these differences, while a value other than one indicates that the change is capable of influencing emission, either by enhancement or suppression.

Plasma conditions were evaluated by means of determining the electron density. Electron density was calculated using the two-line approach described in equation five.

The lines were selected because they meet important already outlined criteria, section 2.2.4, and

also because the use of $\mathrm{MgII} / \mathrm{MgI}$ ratios has been reported in this literature ${ }^{47,58}$ for making assumptions of plasma conditions. Table 10 contains information pertaining to the selected emission lines used for these calculations.

Table 10: Important variable information for electron density calculations ${ }^{15}$ 


\begin{tabular}{|c|c|c|c|c|}
\hline Analyte & Wavelength (nm) & $\mathbf{g}_{\mathbf{k}}$ & $\mathbf{A}_{\mathbf{k i}}\left(\mathbf{s}^{-\mathbf{1}}\right)$ & Excitation Potential (eV) \\
\hline $\mathrm{Mg} \mathrm{II}$ & 279.553 & 4 & $2.68 \times 10^{8}$ & 4.44 \\
\hline $\mathrm{Mg} \mathrm{I}$ & 285.213 & 3 & $4.91 \times 10^{8}$ & 4.34 \\
\hline
\end{tabular}

\subsection{Results and Data Analysis}

\subsubsection{Influence of EIEs and NEIEs on Ionic and Atomic Line Response}

To evaluate the response of analytes spanning a wide range in IPs some emission lines were first selected that covered each of the ranges of the IPs listed above. Results were evaluated utilizing the relative intensity. Figures 12 and 13 depict results for the atomic and ionic emission lines, respectively. In each instance the matrix element was delivered via the matrix tubing at $0.1 \%$ concentration.

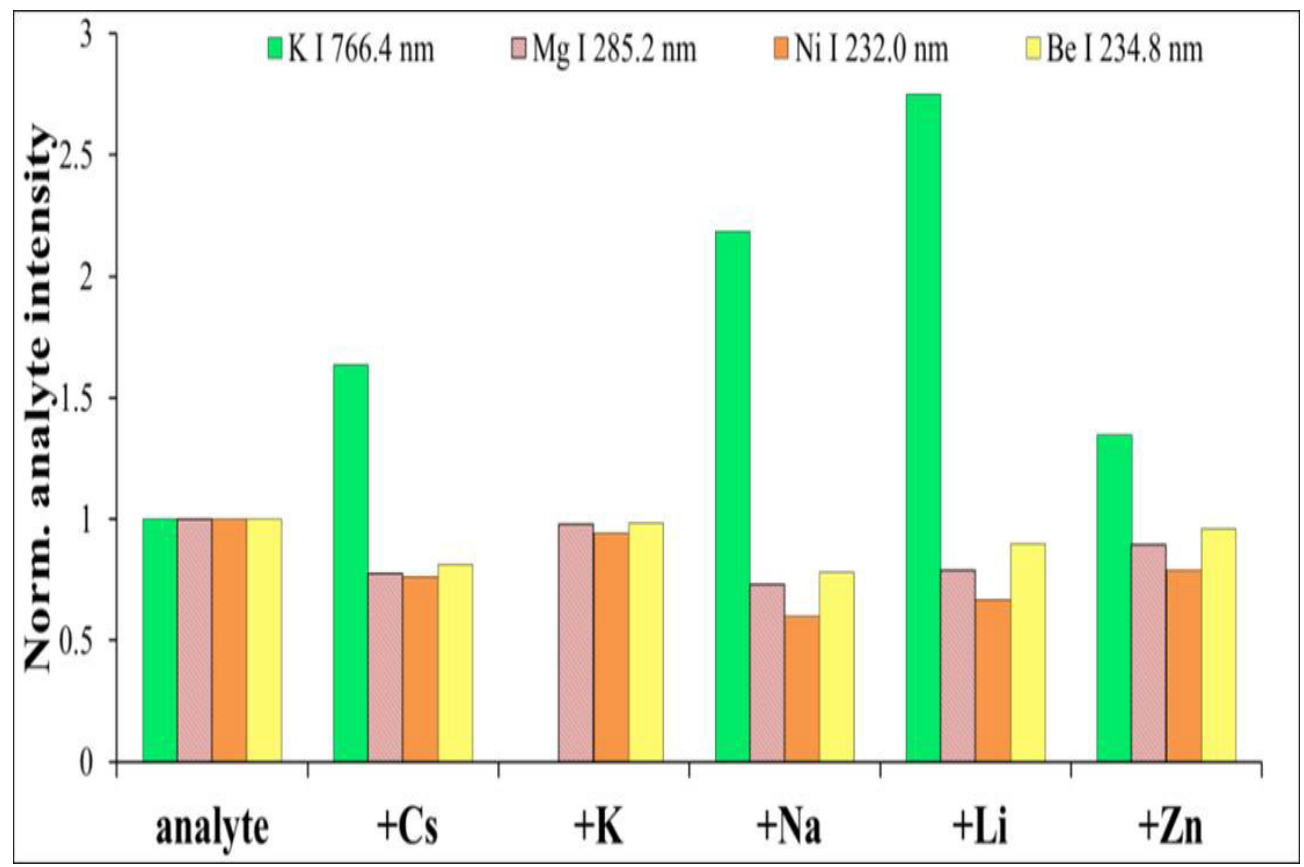

Figure 12: Atomic emission line response in the presence of matrix constituents. 


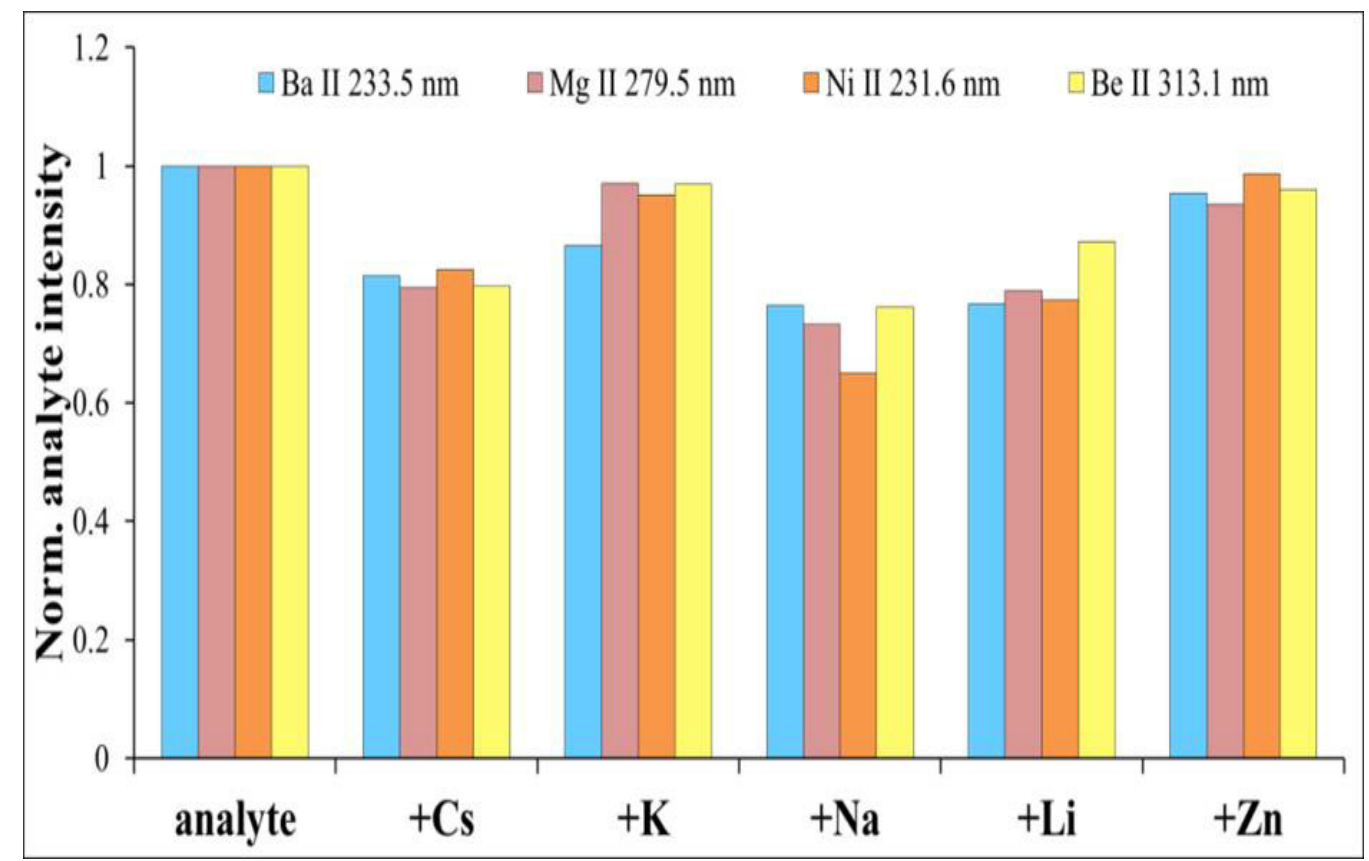

Figure 13: Ionic emission line response in the presence of matrix constituents.

Low IP matrix constituents hindered the detectability of ionic species while all matrices enhanced low IP atomic emission processes (K (I) $766.49 \mathrm{~nm}$ ). The performance in the presence of Cs, $\mathrm{K}$ and $\mathrm{Na}$ was expected and has been reported throughout the literature ${ }^{49,51,59-63}$. However, the presence of a NEIE like Zn did not significantly impact ionic or atomic emission processes. Unlike EIEs, the presence of an NEIE did not significantly perturb ionic and atomic emission processes. It was hypothesized that the ionization mechanism of NEIEs differs from EIEs and therefore does not produce an increase in the population of electrons in the plasma. The hypothesis was corroborated by plotting the calculated electron densities versus the concentration of the matrix (EIE or NEIE). Figure 14 confirms the hypothesis that NEIEs do not affect the electron density to result in the shift of equilibrium that EIEs produce. 


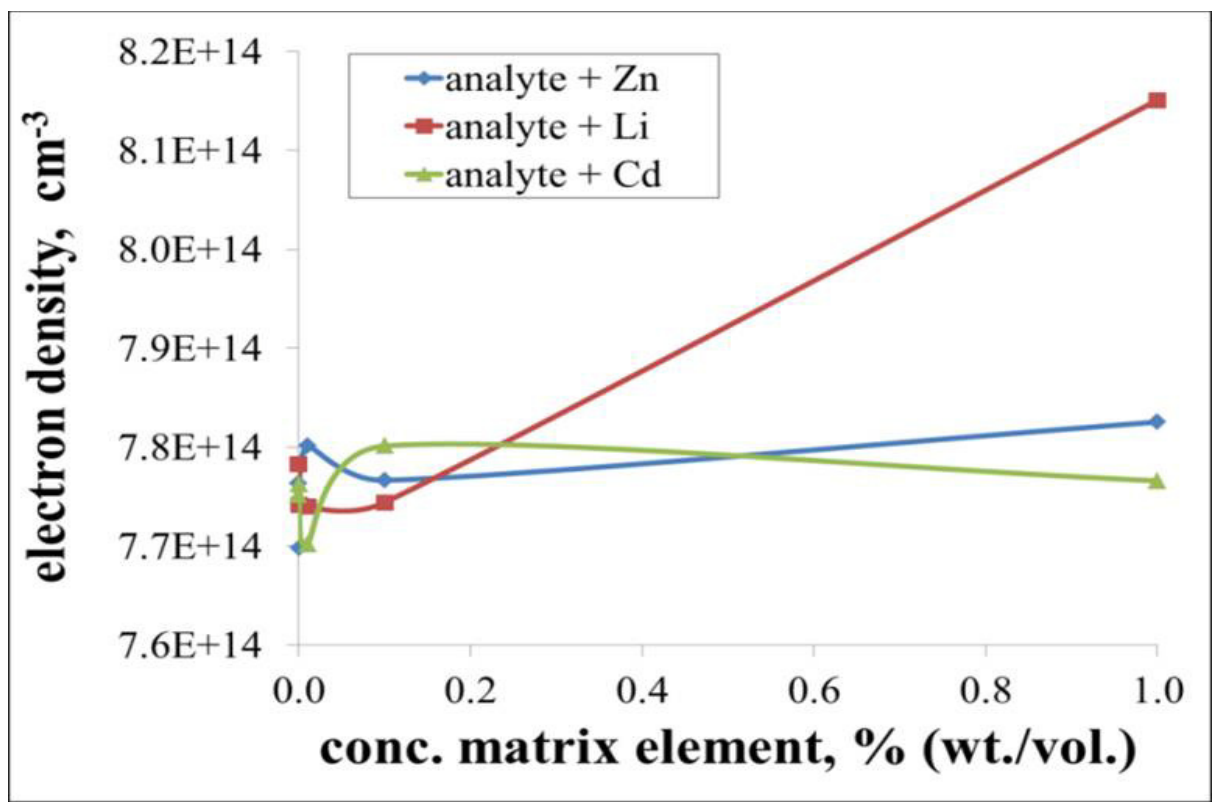

Figure 14: Change in electron density in the presence of different matrix constituents.

The energy of NEIEs is similar to that of Ar metastable species. Charge and energy transfers are most prominent among matched or similarly matched species. However, then it would be expected that NEIEs achieve ionization through Ar metastable interactions. However, the plasma interactions of $\mathrm{Mg}$ have been thoroughly evaluated ${ }^{39,40,64,65}$ and rely on interactions with both $\mathrm{Ar}$ and Ar metastable species. Therefore, if the ionization of NEIEs also relied on Ar-related interactions then the $\mathrm{MgII} / \mathrm{MgI}$ ratio should be affected as $\mathrm{Zn}$ content in the matrix increased. Because this did not occur it infers that either $\mathrm{Zn}$ utilizes an independent pathway for ionization, or the excited Ar species are being replenished at a faster rate than they are being depleted by $\mathrm{Mg}$, $\mathrm{Zn}$ and even other plasma species. Regardless of the actual mechanism, the equilibrium of the plasma conditions remains undisturbed.

The use of both EIEs and NEIEs together was also considered to see if the effects of the EIEs could be canceled out or reduced. The presence of EIEs and NEIEs together at the same concentration was evaluated using the influence on plasma conditions. The comparison was made in the presence of just an EIE $(\mathrm{K})$ at the same concentration. While figure 15 does show that the 
presence of $\mathrm{Zn}$ does somewhat reverse the escalation in electron density this could be due more to the change in transport efficiency of the analyte $(\mathrm{Mg})$ and matrix constituents $(\mathrm{K}+\mathrm{Zn})$. By adding $\mathrm{Zn}$ to the matrix solution the amount of $\mathrm{K}$ reaching the plasma decreases which could result in a lower electron density as well. Because of this uncertainty, NEIEs are considered to have no influence of plasma conditions in the presence of EIEs.

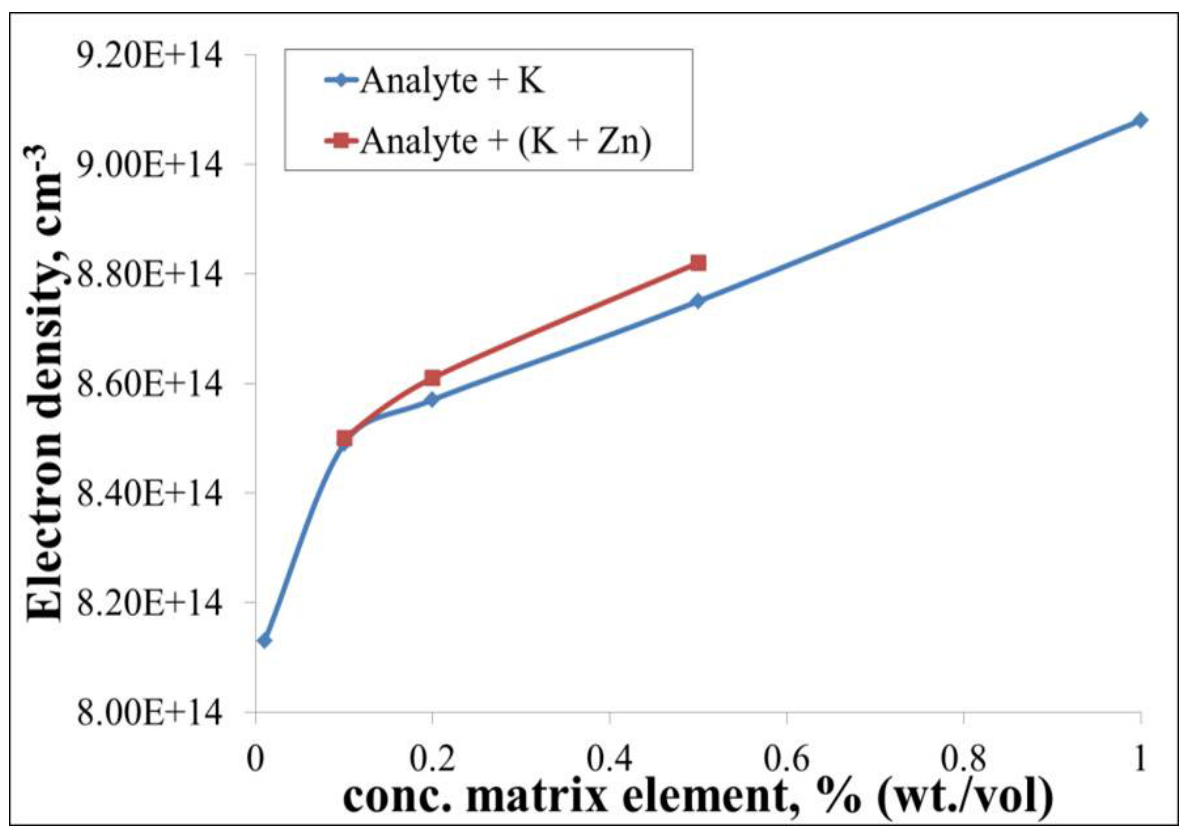

Figure 15: Change in electron density in the presence of EIEs and NEIEs.

Because the energy of NEIEs, particularly $\mathrm{Zn}, \mathrm{Cd}$ has been excluded because of its environmental and health hazards, is similar to $\mathrm{Ar}$ - it is also hypothesized that $\mathrm{Zn}$ is conducive to energy and charge transfers of analytes analogous to argon. In order to confirm this, single element analyte solutions were aspirated with a $0.1 \% \mathrm{Zn}$ matrix. Results were compared on the basis of $1^{\text {st }}$ and $2^{\text {nd }}$ IP of the analyte to determine what, if any, effect $\mathrm{Zn}$ had on the relative intensity and thus also determine the potential ability to conduct charge and energy transfers. Figure 16 depicts the results obtained on the basis of $1^{\text {st }}$ ionization potenetials. Little enhancement 
is observed with a gradual increase in depletion as the $1^{\text {st }}$ IP increases. However, even with a depletion, the signal change is less than $10 \%$ in all cases.

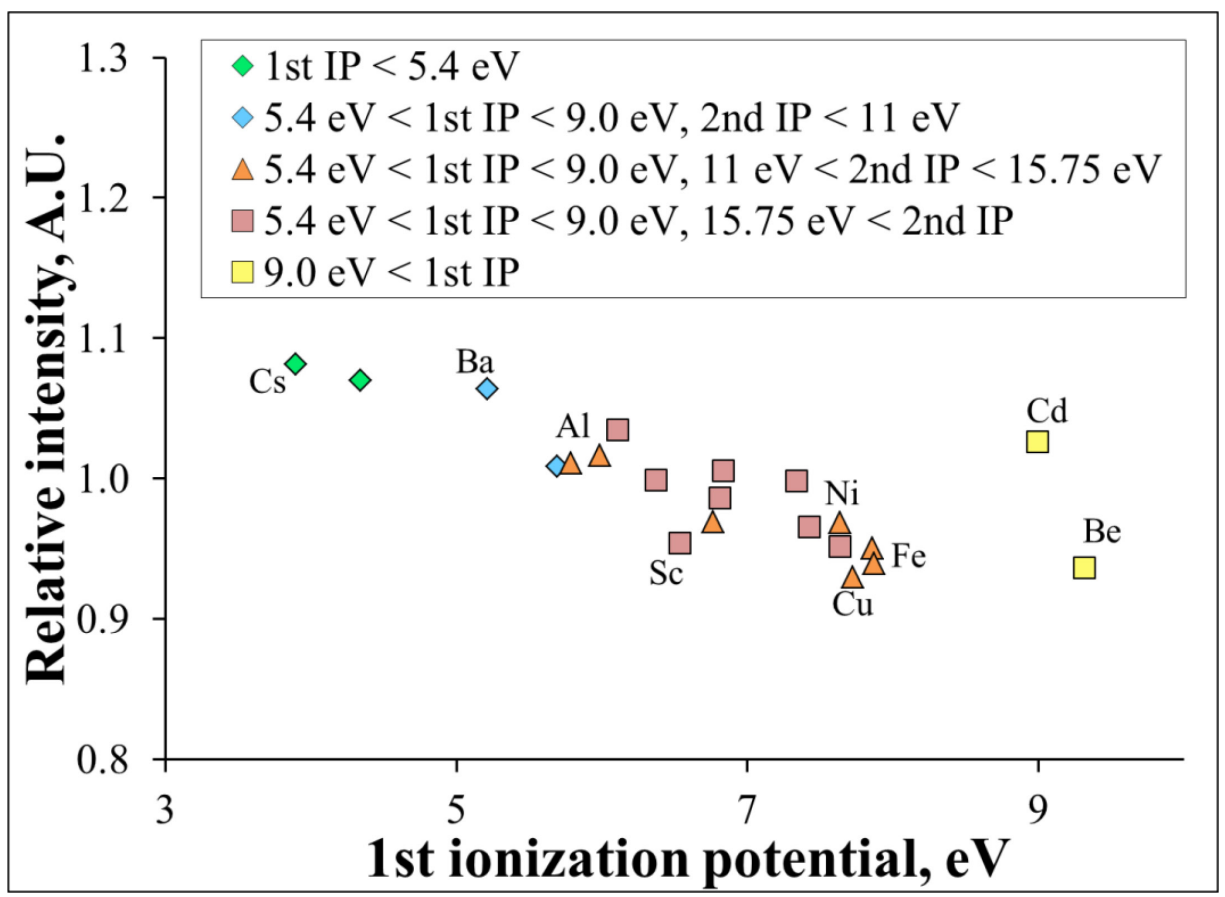

Figure 16: ICP-OES relative intensity trends in relation to $1^{\text {st }}$ IP in the presence of $\mathrm{Zn}$.

Figure 17 depicts the relative intensity results as they pertain to $2^{\text {nd }}$ IP values. Slight enhancement in the relative intensity is observed for analytes with $2^{\text {nd }}$ IPs around the $1^{\text {st }}$ IP of zinc. While not strong, this does suggest the presence of an increase in charge and energy transfers. For other $2^{\text {nd }}$ IPs analytes, relative intensity is approximately 1 with slight depletions of up to $10 \%$. Some elements have been identified on both figure 16 and 17 . What is interesting is that regardless of atomic or ionic species the element performs similarly. This suggests that while energy requirements are important, other unknown mechanisms are also at play. Because no consistent enhancements were observed infers that any role $\mathrm{Zn}$ plays in charge transfer in minimal. At this time further exploration of the actual mechanisms could not be confirmed. 


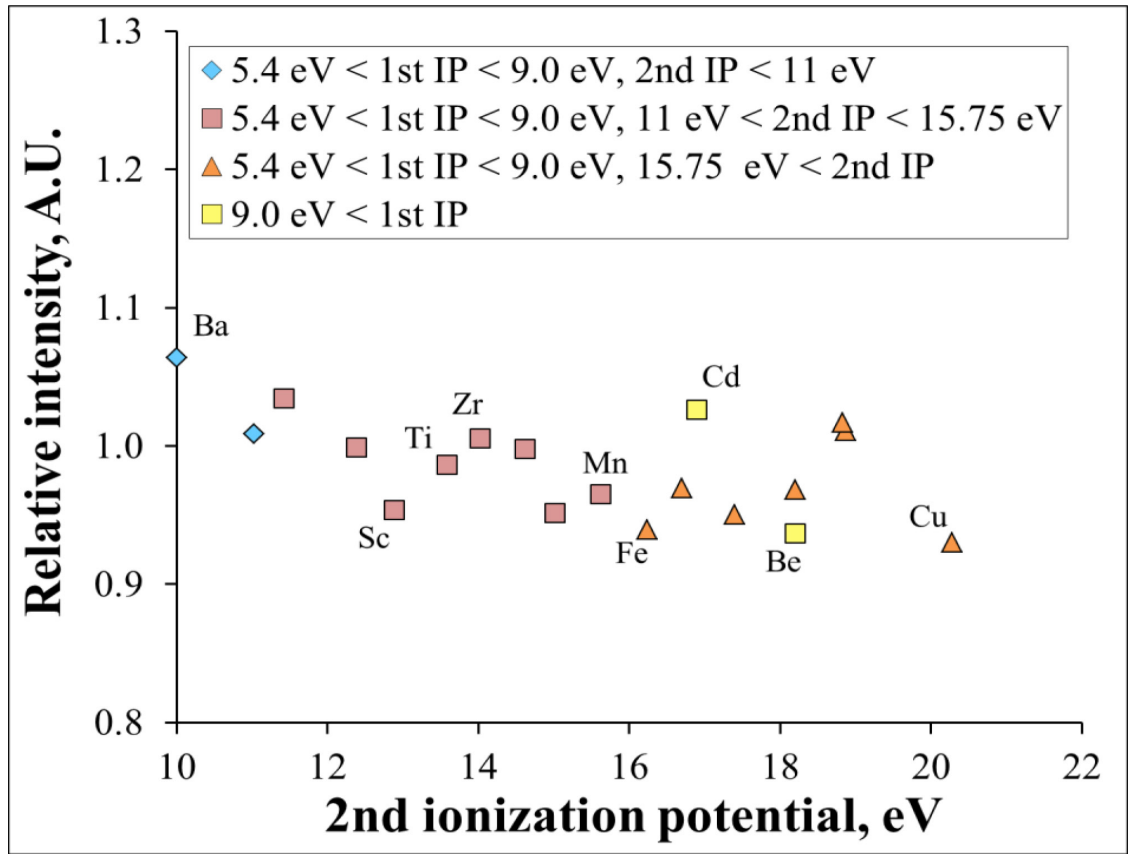

Figure 17: ICP-OES relative intensity trends in relation to $2^{\text {nd }}$ IP in the presence of $\mathrm{Zn}$.

As shown in figure 17 ionic depletion is at least $20 \%$ in the presence of EIEs, twice that in the presence of NEIEs. The smaller decrease in signal shows that the buffering capabilities for single element comparisons are improved over EIEs in the same situation.

\subsubsection{Zinc as a Matrix Buffer}

Other elements have been shown to induce matrix effects ${ }^{45,66}$ including REEs and elements that have low second ionization potentials. Because $\mathrm{Zn}$ has a similar IP as both of these it was also evaluated to determine what types of buffering capabilities it could provide to combat the suppression.

Previous work has suggested that EIEs can be used to buffer solutions, mitigating other matrix effects $^{67}$. However, the current work suggests that using a high IP element that does not shift the plasma equilibrium is a more effective manner to buffer the plasma without depletion in the ionic species. 
To further explore the potential of $\mathrm{Zn}$ buffering in ICP-OES single element comparisons were also made in the presence of other matrix effects. However, because most analytical analysis does not involve single element detection a more stringent evaluation concerning the analytical performance utilizing matrix buffers was also performed.

Magnesium (200 ppbs) in solution with $0.01 \%$ of the matrix effects with aspirated with $0.8 \mathrm{M}$ $\mathrm{HNO}_{3}$ or $0.1 \% \mathrm{Zn}$ in the matrix tubing line. Results have been reported as a percent change in signal comparing analyte with matrix effect, to analyte with matrix effect and zinc. Table 11 presents the buffering abilities of $\mathrm{Zn}$ in some instances.

Table 11: Single analyte performance in the presence of $\mathrm{Zn}$ and other potential matrix effects (in \% difference)

\begin{tabular}{|c|c|c|c|c|c|c|c|c|c|c|c|}
\hline \multirow{2}{*}{ Analyte } & \multirow{2}{*}{$\mathbf{Z n}$} & \multicolumn{2}{|c|}{ Lanthanum } & \multicolumn{2}{c|}{ Scandium } & \multicolumn{2}{c|}{ Samarium } & \multicolumn{2}{c|}{ Yttrium } & \multicolumn{2}{c|}{ Calcium } \\
\cline { 3 - 11 } & $\mathbf{L a}$ & $+\mathbf{Z n}$ & $\mathbf{S c}$ & $\mathbf{+ Z n}$ & $\mathbf{S m}$ & $+\mathbf{Z n}$ & $\mathbf{Y}$ & $+\mathbf{Z n}$ & $\mathbf{C a}$ & $+\mathbf{Z n}$ \\
\hline $\begin{array}{c}\mathrm{Mg}(\mathrm{I}) \\
285.21 \mathrm{~nm}\end{array}$ & 1.0 & -6.3 & 4.5 & -15.3 & -3.2 & -29.5 & -2.8 & -9.9 & -4.1 & -10.0 & -8.2 \\
\hline $\begin{array}{c}\mathrm{Mg}(\mathrm{II}) \\
279.55 \mathrm{~nm}\end{array}$ & -6.9 & -7.0 & 1.7 & -8.1 & 2.1 & -10.8 & -5.1 & -9.7 & -7.3 & -12.6 & -6.9 \\
\hline
\end{tabular}

The application of $\mathrm{Zn}$ in the presence of REEs and $\mathrm{Ca}$ is able to overcome suppression effects that are produced by these matrix effects. Zinc is able to act as a buffer or stabilizer of the plasma interactions. The results suggest that $\mathrm{Zn}$ combats these matrix effects because it participates in the same ionization mechanisms as the matrix effects in table 11. Present in much higher concentration, Zn overwhelms the ionization pathway(s), reducing the extent of REEs and Ca in the plasma for subsequent effect on other analytes. Although the matrix effects, particularly REEs, may not participate directly in the same ionization mechanism as the analytes, several potential routes of ionization for elements with partially filled d-shells reduce available gas molecules and $\mathrm{e}^{-}$for energy transfer to the analyte. The presence of $\mathrm{Zn}$ reduces bias in analytical 
measurements, overcoming suppression upwards of $-28 \%$ to yield suppression typically less than $5 \%$. This is a significant improvement for single element detection.

\subsubsection{Implications of Buffering on Analytical Performance}

The applicability of buffering in the presence of several other constituents has been performed by determining the precision (\%RSD) and accuracy (\%bias) for calibration certification verification (CCV) standards for a Mg calibration curve (1ppb-100ppb). Two CCVs of 20 ppbs and $70 \mathrm{ppbs}$ were analyzed to compare the effects at low and high concentrations. The calibration curve was comprised of solely the analyte, while the CCVs contained additives that were used to evaluate the performance of the buffer. Elements responsible for matrix effects as well as a mock matrix of several other elements (200 ppb P, 20 ppb Sr, 50 ppb Ba and 100 ppb Fe), labeled "elements" in table 12 are used as additives. The Mg (I) $285.21 \mathrm{~nm}$ emission line results are presented.

Bias improves in the presence of $\mathrm{Zn}$ at $0.1 \%$ and $1.0 \%$ over the use of EIEs as buffers. However, the improvement at $1.0 \%$ is less than that obtained at $0.1 \% \mathrm{Zn}$. The difference is most likely the result of high concentrations inadvertently changing the surface tension and viscosity of the solution. Larger droplets are then produced in the ICP-OES nebulizer, which yields a poorer ionization in the plasma. Therefore, while $\mathrm{Zn}$ as a buffer can mitigate the effects from other elements or matrix species, there is a point at which the concentration should not be exceeded. On the basis of the observations, a $\mathrm{Zn}$ buffer provides optimal functioning at $0.1 \%$ concentration. 
Table 12: Analytical performance in the presence of matrix effects and buffers

\begin{tabular}{|c|c|c|c|c|c|c|c|}
\hline \multirow[b]{2}{*}{ Matrix } & \multirow[b]{2}{*}{ Analyte } & \multicolumn{3}{|c|}{$\mathrm{Mg}, 20 \mathrm{ppm}$} & \multicolumn{3}{|c|}{$\mathrm{Mg}, 70 \mathrm{ppm}$} \\
\hline & & $\begin{array}{c}\text { Conc., } \\
\text { ppm }\end{array}$ & $\%$ RSD & $\%$ Bias & $\begin{array}{c}\text { Conc., } \\
\text { ppm }\end{array}$ & $\%$ RSD & $\%$ Bias \\
\hline \multirow{4}{*}{$\begin{array}{l}0.8 \mathrm{M} \\
\mathrm{HNO}_{3}\end{array}$} & Analyte & 19.0 & 4.6 & -5.1 & 65.5 & 3.1 & -6.5 \\
\hline & $+0.01 \% \mathrm{Y}$ & 19.2 & 3.0 & -4.0 & 64.8 & 2.2 & -7.4 \\
\hline & $+0.01 \% \mathrm{Ca}$ & 19.1 & 3.0 & -4.5 & 67.0 & 2.9 & -4.2 \\
\hline & + elem. & 19.5 & 3.4 & -2.5 & 66.7 & 1.6 & -4.7 \\
\hline \multirow[t]{4}{*}{$0.1 \% \mathrm{Zn}$} & Analyte & 19.2 & 4.0 & -4.0 & 68.5 & 3.3 & -2.1 \\
\hline & $+0.01 \% \mathrm{Y}$ & 19.4 & 4.4 & -3.0 & 66.2 & 2.8 & -5.4 \\
\hline & $+0.01 \% \mathrm{Ca}$ & 19.3 & 4.8 & -3.5 & 68.9 & 3.6 & -1.6 \\
\hline & + elem. & 19.4 & 2.2 & -3.0 & 68.4 & 2.7 & -2.3 \\
\hline \multirow[t]{4}{*}{$1.0 \% \mathrm{Zn}$} & Analyte & 19.0 & 4.5 & -5.0 & 67.3 & 3.5 & -3.9 \\
\hline & $+0.01 \% \mathrm{Y}$ & 18.4 & 9.1 & -8.0 & 64.9 & 1.6 & -7.2 \\
\hline & $+0.01 \% \mathrm{Ca}$ & 19.1 & 6.5 & -4.5 & 67.1 & 4.1 & -4.1 \\
\hline & + elem. & 19.5 & 5.4 & -2.5 & 67.2 & 1.9 & -4.0 \\
\hline \multirow[t]{4}{*}{$0.1 \% \mathrm{Li}$} & Analyte & 18.4 & 4.2 & -8.0 & 68.8 & 4.2 & -1.7 \\
\hline & $+0.01 \% \mathrm{Y}$ & 15.1 & 4.1 & -24.5 & 67.6 & 4.1 & -3.4 \\
\hline & $+0.01 \% \mathrm{Ca}$ & 19.0 & 1.1 & -5.1 & 67.2 & 1.1 & -4.0 \\
\hline & + elem. & 20.1 & 1.5 & -0.5 & 67.9 & 1.5 & -3.0 \\
\hline \multirow[t]{4}{*}{$1.0 \% \mathrm{Li}$} & Analyte & 19.2 & 2.9 & -4.0 & 63.3 & 0.8 & -9.5 \\
\hline & $+0.01 \% \mathrm{Y}$ & 19.4 & 6.7 & -3.0 & 62.9 & 1.6 & -10.1 \\
\hline & $+0.01 \% \mathrm{Ca}$ & 19.2 & 4.3 & -3.0 & 64.1 & 3.9 & -8.4 \\
\hline & + elem. & 19.2 & 1.9 & -3.0 & 64.5 & 4.4 & -7.9 \\
\hline
\end{tabular}

\subsection{Discussion and Conclusions}

The work conducted here provides an additional, unexplored method to mitigating matrix effects of REEs and low $2^{\text {nd }}$ IP elements in inductively coupled plasma optical emission spectrometry (ICP-OES). Based on analytical performance and plasma conditions, $\mathrm{Zn}$ is a suitable plasma stabilizer or buffer. The use of NEIE is able to provide improved performance at a lower concentration than required when the use of Cs and other EIEs has been attempted for buffering (present at $1-10 \%)^{67}$. Such high concentrations reduce the lifetime of ICP-OES consumables as well as torch components and plasma viewing windows.

In addition, $\mathrm{Zn}$ improves the sensitivity of the method. When severe matrix effects are present, radial, or side viewing of the plasma is used to reduce the effect. However, this has an opposite effect on the sensitivity of the method. Depending on the strength of the emission, the LOD of the 
method can be much higher than with axial viewing. The use of $\mathrm{Zn}$ allows for the analysis to be conducted using axial viewing. Therefore, any decrease in performance as a result of a lower transport efficiency with the addition of a buffer, is still more sensitive than radial viewing. In addition, some elements do not possess the sensitivity in general emission processes to be detectable in trace quantities using radial viewing. Zn buffering is able to provide a solution to this, increasing the versatility of analytical analysis for complex matrices being analyzed by inductively coupled plasma optical emission spectrometry (ICP-OES).

The research presented recommends the use of $\mathrm{Zn}$ at $0.1 \%$ for buffering matrices that contain REEs and/or low second ionization potential elements. The matrix should be introduced using a similar sample introduction system as employed in this research. The approach allows for the matrix to be kept entirely separate from the sample being analyzed, eliminating contamination of the sample which is advantageous when further analyses could be required. The current studies provide a simple solution to combating some prominent matrix effects and can be easily implemented in ICP-OES applications.

\subsection{INFLUENCE OF GAS COMPOSITION ON LA-ICP-OES PERFORMANCE}

\subsection{Overview}

Several studies utilizing laser ablation coupled to ICP-OES (LA-ICP-OES) have been conducted to evaluate the implications of central channel gas flows in analytical analysis. Both gas composition and flow rates of the nebulizer and ablation cell sweep gas are important to the generation of a stable plasma, and also strong, reproducible emission signals for trace elemental analysis. Gas composition and the total ICP torch central channel gas flow rate was assessed by analyzing glass and pelleted unprocessed cotton. The matrices are very different in physical and chemical characteristics which yield different responses to laser ablation, both in efficiency and rate, making them suitable test matrices for making comparisons and generalizations of LA-ICP- 
OES operating conditions. The application of these matrices addresses the applicability of providing uniformity to some essential LA-ICP-OES parameters. Optimal total central channel gas flow rate was determined to be $0.5 \mathrm{~L} / \mathrm{min}$, comprised solely of Ar. Lower overall gas flow rates resulted in higher emission intensities with better precision ( $>1 \%-8 \% \mathrm{RSD})$. In addition to gas-related parameters, other studies were conducted regarding the influence of the process of mixing a two-component central channel gas, the volume of the ablation cell in respect to gas flow, the effect of transport tubing length as well as the quality of ablated material generated with the different operating conditions that were considered.

\subsection{Introduction}

Laser ablation coupled to ICP-OES (LA-ICP-OES) for analytical analysis has been demonstrated as a suitable technique for various applications ranging from research ${ }^{68}$, industrial ${ }^{69}$ and environmental ${ }^{70}$ among others. Although not as popular as laser ablation inductively coupled plasma mass spectrometry (LA-ICP-MS), reporting of this technique are increasing, most likely as a consequence of improvements in instrumentation over mass spectrometry.

The application of laser ablation allows for the analysis of solid samples by means of introducing small amounts of ablated material into the ICP, which is followed by detection of atomic and ionic emission processes taking place in the plasma.

Both LA-ICP-MS and LA-ICP-OES utilize the same sample introduction of ablated material or mass, as well as similar instrumental front-ends, or ICP designs. Laser ablation, in both instances can be accomplished using the same laser ablation units. The application of laser ablation has been employed in both techniques to simplify sample preparation, eliminating the need for lengthy, caustic acid digestions, which are often necessary for solution-based sample introduction in both ICP techniques. 
Because of the maturity of LA-ICP-MS, several studies have been conducted using this technique in order to explore optimal working conditions, regardless of matrix ${ }^{71-75}$. The studies involve the evaluation of specific operating conditions pertaining to the influence of central channel gas flow rate and central channel gas composition on analytical performance of the technique (sensitivity, LOD) and the quality of ablated material and efficiency of transport to the inductively coupled plasma (ICP).

Here, the central channel gas has been defined to include what is referred to as the nebulizer gas, and also the sweep gas, which may be any additional gas or flow which is used to sweep the ablation cell and aid in the transportation of the ablated mass to the ICP torch. The studies conducted by LA-ICP-MS in regard to these parameters have concluded that the use of helium (He) as the sole composition of the sweep gas, which is then combined with the Ar nebulizer gas, consistently resulted in smaller particle size distributions rather than the generation of aggregates in the ICP due to differences in the thermal conductivity properties of He and argon ${ }^{72}$. The research suggested that the higher thermal conductivity of He coupled with the smaller laserinduced plasma formed in the environment yields a faster sweep of the ablated material out of the ablation cell before the formation of aggregates could occur ${ }^{72}$.

Laser ablation inductively coupled plasma mass spectrometry (LA-ICP-MS) studies have also been conducted varying the tubing length to determine the impact on particle size distributions that reach the ICP torch $^{72}$ as the tubing connecting the ablation cell to the torch is varied. The results presented were not influenced by tubing length.

The studies described above stress the importance of 1) instrumental setup, 2) gas flow and 3) gas composition in achieving optimal sensitivities regardless of the composition of the matrix being analyzed. The literature is in agreement that He-Ar mixtures in LA-ICP-MS as the carrier gas composition promotes sensitive measurements ${ }^{76}$. 
Fundamental research has also been conducted using LA-ICP-OES to evaluate central gas composition as it pertains to different matrix effects ${ }^{35,77,78}$. In addition, the parameters that are reported for LA-ICP-OES applications vary from those utilized in the investigation of plasma interactions. The discrepancy in these parameters suggest that either 1) optimal conditions are greatly influenced by the matrix being analyzed using LA-ICP-OES and thus cannot be generalized or 2) robust plasma conditions can be achieved with several different operating conditions which may or may not have an influence on the analytical sensitivity of the analysis. Studies previously conducted by LA-ICP-OES using He, Ar, He-Ar or Ar-He mixtures have evaluated the presence and influence of matrix effects that may interfere with analyte analysis, however have not considered the generalization of flow rate and composition in regards to the analytical performance of the technique.

As a result of the differences in detection principles in LA-ICP-MS and LA-ICP-OES, it is hypothesized that while these parameters are important in both instances, the optimal conditions will vary in order to produce the necessary robust plasma conditions needed for each respective technique. A robust plasma, regardless of ICP technique being considered, specifies conditions in which the response of an analyte at a fixed concentration is not influenced by changes in matrix conditions $^{79}$. A robust plasma has been measured using $\mathrm{MgII} / \mathrm{MgI}$ intensity ratios and is achieved in ICP-OES when the ratio exceeds $8^{80}$ in some reportings and is as high as $13^{81}$ in others. The identified range will be used to verify that a robust plasma has been obtained for the operating conditions studied. The ratio of ionic-to-atomic line intensities are also used to assess plasma conditions, i.e. electron density and plasma temperature ${ }^{82}$. In addition the experimental ratio can be compared to the calculated $\mathrm{MgII} / \mathrm{MgI}$ ratio to determine what, if any, deviation from local thermodynamic equilibrium is present ${ }^{35}$. 
Studies presented in this chapter evaluate the influence of central channel gas flow rate and composition, ablation cell volume and transport tubing length on trace analytical analysis. The design of the studies is similar to that which has what has been previously described by LA-ICPMS in order to bridge research and applications for laser ablation inductively coupled plasma optical emission spectrometry (LA-ICP-OES). The studies will be evaluated using two test matrices: silicate glass SRMs and a pelleted unprocessed cotton. As demonstrated in chapter 2, these matrices differ greatly in the matrix composition and physical property as well as in the response to ablation, and thus serve as good matrices for evaluating the generalization of some critical LA-ICP-OES parameters.

\subsection{Materials and Methods}

\subsubsection{Description of Samples and Sample Preparation}

\subsubsection{Cotton Matrix}

The cotton SRM and in-house standard previously described in section 2.3.1.1 is being used. Sampling strategies and pellet preparation of this matrix is addressed in chapter 6 of this dissertation for reference, see section 6.3.1.1-.4.

\subsubsection{Glass Matrix}

All glass SRMs utilized in the studies have been previously described in section 2.3.1.2. Glass SRMS analyzed here were of full thickness. Although not all results for each glass standard are presented here, all SRMs were utilized to confirm observed trends. The glass SRMs were also selected because of their different physical properties and possible different interactions in the plasma, as well as differences in the elemental composition. There are large differences in trace analytes and calcium $(\mathrm{Ca})$ concentration. The influence of low $2^{\text {nd }} \mathrm{IP}$ species like $\mathrm{Ca}$ in the

plasma has been previously studied ${ }^{35,77}$. This work has shown that the matrix effect can be greatly 
reduced when He is used to completely replace Ar in the central channel ${ }^{35}$. However, the implications of an entirely central channel of He have not been addressed in regard to the sensitivity of the method for trace analysis. Here, the use of the glass matrix allows for the analytical performance and plasma conditions to be compared.

\subsubsection{Principles of Laser Ablation for ICP techniques}

The term laser ablation broadly describes the use of a laser to interact with a solid sample to remove a finite amount of mass ${ }^{8}$. The mass removed can be from a single location, resulting in a crater, or from a new location each time the laser comes in contact with the solid, yielding a line of removed mass. The mass removed is relatively small, hundreds of nanograms to low micrograms as measured by using crater volumes determined by a digital microscope, similarly to what has been presented in chapter 2 of this dissertation.

Laser ablation is the first step for several analytical approaches. It is the first step in LIBS as well as in laser based solid sampling ICP techniques. Although these techniques differ in that the subsequent ionization and plasma processes take place on the sample surface in LIBS whereas in ICP the ablated material has to be transported to the plasma. This chapter focuses on the latter.

Laser ablation for ICP techniques is accomplished using commercial setups. A laser ablation unit consists of a laser, an ablation cell, a camera for viewing the sample and tubing to transport the ablated material to the ICP. An additional scavenger gas is used to sweep the ablated material to the ICP torch for ionization and detection. A significant amount of research has been dedicated to the selection of laser specifications and the design of laser ablation units for optimal stoichiometric sample ablation ${ }^{83-86}$. Stoichiometric ablation refers to the generation of ablated

material that is representative of the sample composition ${ }^{86,87}$. Non-stoichiometric ablation, usually referred to as fractionation, can result from various points in the sampling process - laser 
interaction, sample transport or ICP ionization. It is necessary then to account for and minimize possible sources of fraction. The selection of an optimal laser can influence and reduce the event of fractionation.

The most important laser parameter is the irradiating wavelength. The use of a high energy wavelength (UV) laser minimizes fractionation ${ }^{88,89}$. In addition the shorter the laser pulse (nanosecond vs femtosecond) the less fraction as well ${ }^{88-91}$. These two laser parameters influence the particle size distributions and the amount of mass ablated ${ }^{92}$. However, stoichiometric ablation can still be achieved with nanosecond laser ablation ${ }^{93}$ and the ablation quality gained with femtosecond laser ablation is often outweighed by the increase in cost.

Among the main advantages listed earlier for laser ablation a major ability of the technique is the removal and analysis of such small amounts of material to be considered a nondestructive technique. However, this can also be a downfall. Because laser ablation samples such a small portion of the material it is considered a micro chemical technique ${ }^{90}$ for both ICP techniques and LIBS. To overcome this, several replicates are analyzed in order to obtain a more representative characterization of the bulk material; though this can be difficult to accomplish for small, irregular shaped samples. Sampling strategies have been developed to account for this property of laser ablation ${ }^{94,95}$. Another disadvantage to the laser ablation of solid material is the difficulty in achieving quantitative results. Quantitative results require matrix-matched or matrix-similar standards. Ablation properties vary according to changes in the matrix, so standards need to be similar in bulk characteristics to yield similar ablation to the sample. This may limit the applicability of this technique for widespread applications. 


\subsubsection{LA-ICP-OES Experimental Design}

\subsubsection{Laser Ablation Units}

Laser ablation analyses were performed using one of two laser ablation units: a UP-213 (New Wave Research Inc., Fremont, CA, USA) laser ablation system operating at 213 nm, 4 ns pulse duration, or a Cetac LSX-500 (Cetac Technologies, Omaha, NE, USA) laser ablation system operating at $266 \mathrm{~nm}, 6 \mathrm{~ns}$ pulse duration. Both laser ablation units utilized q-switched, Nd:YAG lasers. A camera was used to focus on the surface of the sample. The camera focus was associated with the laser focus for both laser ablation units.

The ablation cell volume of the UP-213 system was kept constant at $30 \mathrm{~cm}^{3}$ while the LSX-500 cell volume could vary depending on the height of the sample stage utilized. In the studies presented here one of two stages were used with varying heights of $14 \mathrm{~mm}$ high or $21 \mathrm{~mm}$ high. The heights of the stages influence the available cell volume resulting in $63 \mathrm{~cm}^{3}$ or $50 \mathrm{~cm}^{3}$ for the short and medium stage heights, respectively. Unless otherwise stated the medium height samples stage was used with the LSX-500.

Regardless of matrix being analyzed spot ablation mode was used. For glass analysis a $100 \mu \mathrm{m}$ spot size was utilized as it was the smallest laser beam diameter that removed a sufficient amount of mass to detect all elements, both trace and minor constituents, pertinent to the elemental analysis of glass as was previously reported ${ }^{96}$. Cotton analysis employed a $200 \mu \mathrm{m}$ spot size to account for any sample microheterogeneity in the pellet that might be observed and exploited when too small of an area is sampled. The $200 \mu \mathrm{m}$ beam diameter was utilized based on LA-ICPMS analysis of the cotton pellets.

The LSX-500 was also capable of depth profiling which was used in all studies that utilized this ablation unit. The z-stage could be stepped in $0.25 \mathrm{~mm}$ increments. This ability was used for both 
matrices. Depth profiling is used to ensure that the same part of the beam is in contact with the sample as the crater is formed by constantly increasing the height of the same stage throughout the duration of laser ablation.

Laser frequencies of 5 and $10 \mathrm{~Hz}$ were used for cotton and glass analysis, respectively.

Differences in physical properties of the matrices account for the different optimal laser parameters. Optimal output laser energy also varied for each matrix. Glass analysis was always conducted using $100 \%$ laser output energy, regardless of laser ablation unit. Cotton was analyzed using a 90\% laser output on the LSX-500. Less energy was required to remove mass from the less dense, softer cotton pellet.

The duration of laser ablation utilized for each matrix was determined on the basis of the amount of mass removed with each pulse and the time in which it took to ablate enough mass so that at least $20 \mathrm{~s}$ of stable ablation introduction into the ICP could be achieved. This was assessed using the stability of the emission signal obtained. Total ablation times varied as different parameters were considered and are reported for each study.

\subsubsection{ICP-OES Setup and Instrumentation}

The ICP-OES instrument used in these studies was the same Optima 7300 DV (PerkinElmer, Waltham, MA, USA) reported in chapter 3 of this dissertation. Because the general instrumental configuration is similar, this section will focus on the differences in setup and the relevance of these to the research conducted within the scope of the chapter. Important parameters for LAICP-OES method development include integration and read time. These parameters differ depending on the matrix being analyzed and are important to obtaining a meaningful transient signal in LA-ICP-OES. Due to the differences in quality of signal observed with varying the integration time and read time, all studies were conducted with $0.01 \mathrm{~s}$ integration time, $0.1 \mathrm{~s}$ read 
time for cotton analysis, and $0.1 \mathrm{~s}$ integration time and $1 \mathrm{~s}$ read time for glass analysis. The differences in integration and read times for the two matrices are the result of differences in mass ablation rates which influence the ability to produce detectable emission. It is suspected that since less glass material is removed with each laser pulse more time viewing the emission processes is required to detect trace elements. The material is also denser and requires more energy to break into atomic and ionic constituents. Also, it is important to consider that while a faster read time results in more data points this is not critical when the ultimate goal is to use the data to generate a transient signal, section 4.3.6. On the other hand, a fast integration time produced an unstable signal, very sensitive to fluctuation of mass delivery and gas flows. The integration and read times selected gave enough detail in the signal to observe trends in the ablation efficiency and gas flows that would influence the analytical data - not be over exaggerated due to selection of data collection parameters. The numbers of readings made per replicate were kept constant for each study, 100 readings for both cotton and glass, unless otherwise stated. Five replicates were performed on each sample in each study.

A quartz torch allowing for central channel, outer channel and cooling gas flows was used in all experiments conducted. In all studies conducted the plasma gas flow was composed only of Ar at $15 \mathrm{~L} / \mathrm{min}$. The cooling or auxillary gas was also kept constant at $0.5 \mathrm{~L} / \mathrm{min}$ Ar. Only the central channel gas flow was adjusted in both flow and composition. The forward power was maintained at the maximum of the instrument, $1500 \mathrm{~W}$, for all studies unless stated otherwise. The injector for the torch was composed of alumina oxide with an internal diameter of $3 \mathrm{~mm}$. The height above load coil (HALC) used for viewing and detecting the emission processes could be manipulated using the software of the instrument, WinLab32 (PerkinElmer, Waltham, MA, USA). 


\subsubsection{Manipulation of External Gas Introduction}

Only Ar and He have been considered for the makeup of the central channel gas composition. Initial Ar flows were introduced using the nebulizer line from the ICP-OES instrument. If additional Ar was needed or for the introduction of He gas, an external gas line was employed. The flow of the second gas was regulated using an electronic flow regulator (model \# FMA2605A, Omega Engineering Inc., Stamford, CT, USA).

The mixing of two gases or two gas flows was accomplished using a T-type connection made of polypropylene and are meant to fit tubing that had a 1/8" ID (Thermo Scientific, Rochester, NY, USA). Mixing was accomplished in-line with the ablation cell, either before or after the cell. Tygon tubing, formulation R-3603 with a 1/8” ID and 1/4" outer diameter (Fisher Scientific, SaintGobain Performance Plastics, Valley Forge, PA, USA) was used to connect the ablation cell to the torch and all other laser ablation connections.

\subsubsection{Indirect Determination of Particle and Aggregate Size of Ablated Material}

An in-line filter system, figure 18, was used to screen the particle size that was reaching the ICP torch. Single layer of $0.45 \mu \mathrm{m}$ mesh in-line with the tubing that transports the ablated particles to the torch. The filter system was placed in one of two locations, either just outside the ablation cell prior to introduction of the second gas, or following the " $\mathrm{T}$ " and the introduction of the second gas. In order to ensure that the filter system was not influencing the ICP-OES system the back pressure was monitored for the duration of the studies. The back pressure did not fluctuate more than one unit $(1.0 \mathrm{kPa}$ to $2.0 \mathrm{kPa})$.

Because the filters were used as a screen to limit the transport of larger particles this is considered an indirect approach to monitoring the size distribution of the ablated material. A change in emission intensity was considered to reflect the particle size distribution, i.e., a drop in intensity 
suggested larger particles or aggregates. The difference was used to determine the influence the gas composition had on the particle and aggregate distribution. A comparison of no filter, filter before the " $T$ " and filter after the " $T$ " results were used to indirectly determine the influence of gas composition on particle sizes by means of emission intensity.

A direct approach to determining the particle size distribution was also evaluated by using a high magnification microscope. However, initial assessment as to the cleanliness of the mesh exhibited the presence of particulate matter embedded in the fibers. As a result, it was difficult to distinguish between ablated material aggregates and pre-existing particulates in the fiber. It was because of this that an indirect approach measuring change in emission to reflect a change in the size of particles or aggregates reaching the torch was used.

In addition, larger pore filters were also used $(150 \mu \mathrm{m}$ nylon mesh) in single or several layers combinations in the filter system. However, no change in intensity was observed when six layers were utilized, suggesting that this was not sufficiently screening the ablated material.

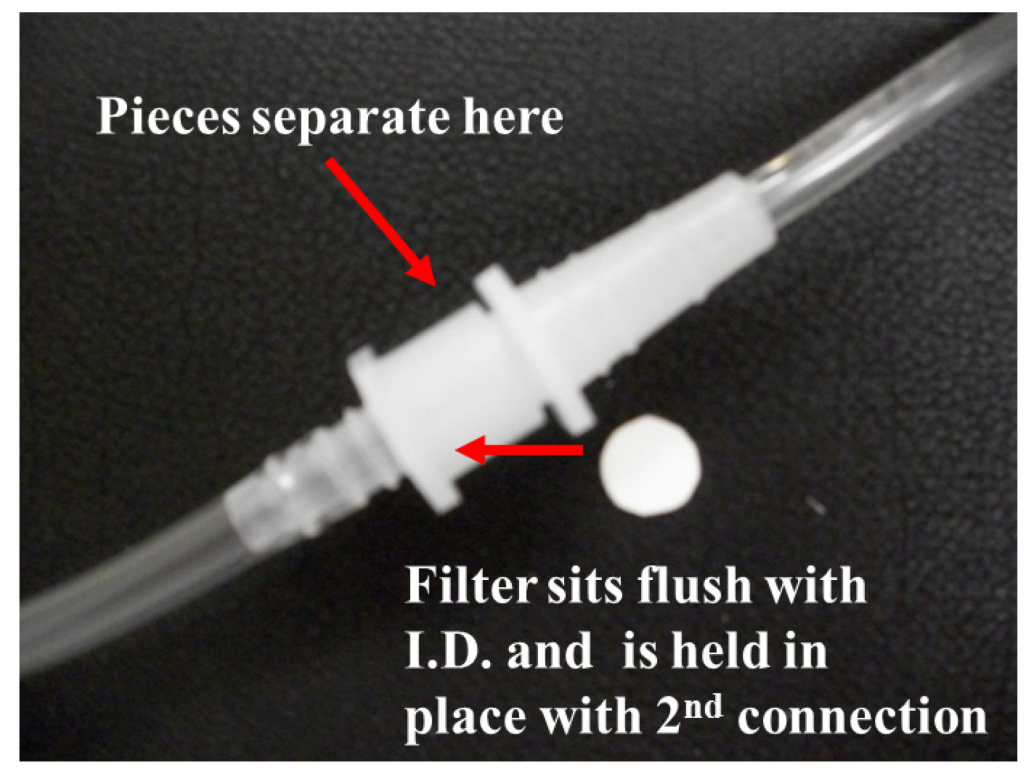

Figure 18: Filter setup for determining particle size effects in LA-ICP-OES. 


\subsubsection{Daily Performance and Quality Control of LA-ICP-OES Setup}

In order to ensure that the overall performance of the LA-ICP-OES setup did not vary significantly day-to-day and to allow for comparisons of studies made on different days the responses of two glass standards were monitored, NIST610 and NIST 612. The SRMs were selected because of the differences in general concentrations of several elements, $\sim 50 \mathrm{ppm}$ and 500 ppm for NIST612 and NIST610, respectively. Regardless of the study being conducted the SRMs were run under the same conditions prior to the start of sample analysis. The parameters for both LA units included 100\% laser energy output, $10 \mathrm{~Hz}$ laser frequency, $100 \mu \mathrm{m}$ spot diameter of the laser beam, line ablation (scanning $10 \mu \mathrm{m} / \mathrm{s}$ ), data collected for 20 replicates using $0.1 \mathrm{~s}$ integration time and $1 \mathrm{~s}$ read time. Both weak and strong emission lines were monitored to ensure adequate sensitivity prior to conducting experiments. The first four data points were used to establish upper $(+2 \sigma)$ and lower $(-2 \sigma)$ control boundaries.

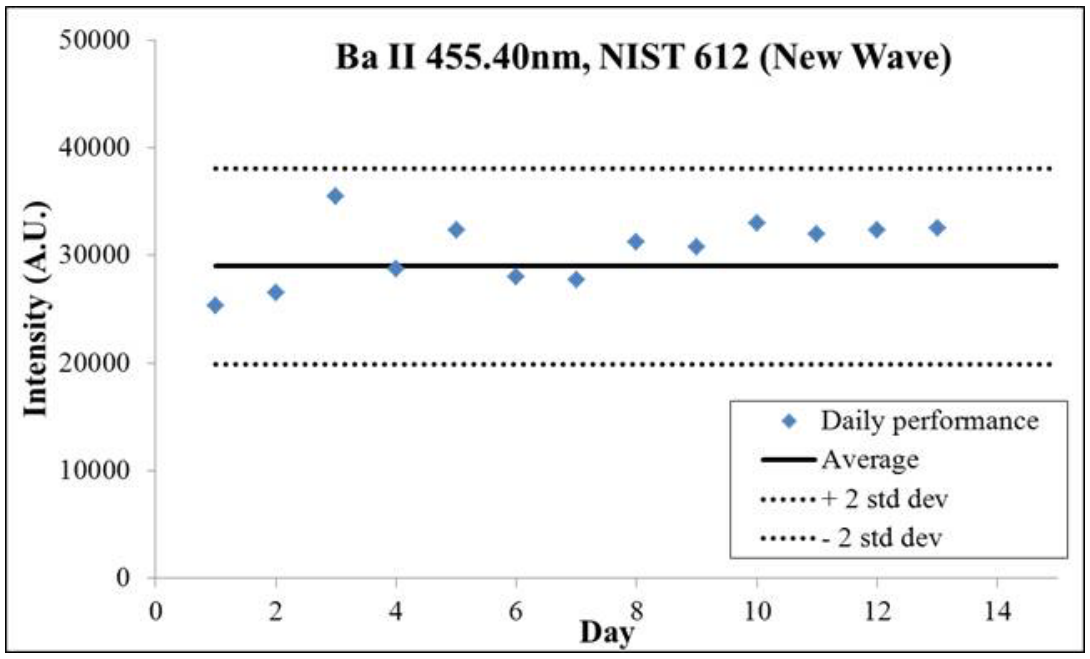

Figure 19a: QC chart for LA-ICP-OES, example 1. 


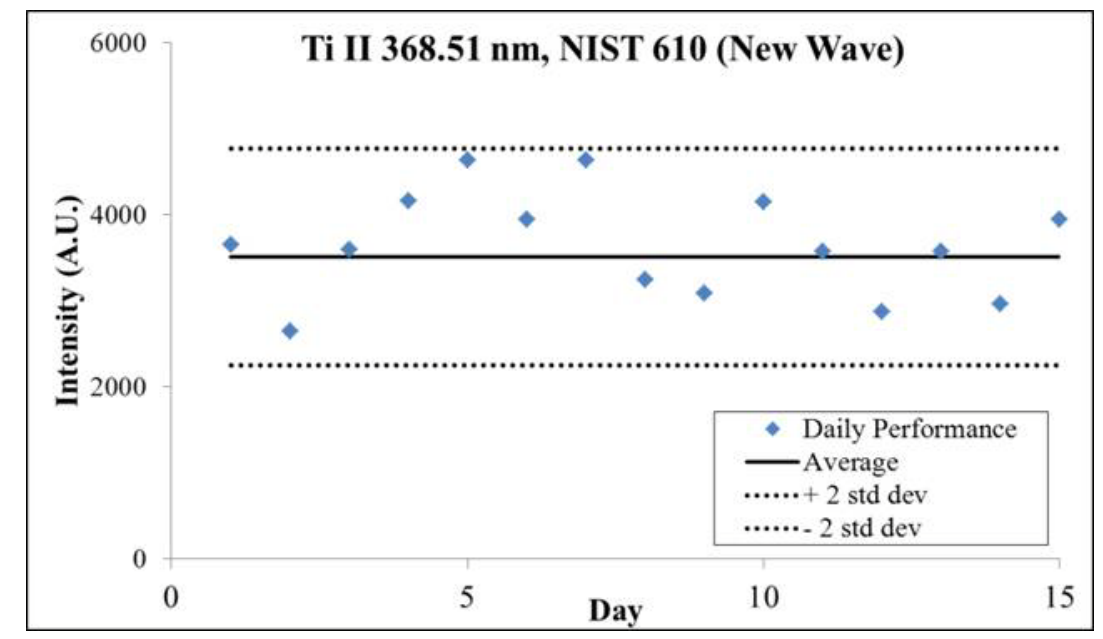

Figure 19b: QC chart for LA-ICP-OES, example 2.

Both low and high concentrations performed well over time. A change in performance was taken to imply maintenance was needed. Similar day-to-day variation was observed when the CETAC unit was used.

\subsubsection{Generation of a Transient Signal}

The data analysis of LA-ICP-OES results involves the assessment of emission signals generated as a function of time before, during and after laser ablation. The ICP-OES software records the emission process as the intensity of the emission line over time. Since laser-based ICP techniques utilize transient signals for further data analysis and interpretation of results the LA-ICP-OES output can be transposed to present a transient signal. A transient signal is the product of comparing a response, in this instance the intensity of emission, on the basis of time, typically reported in seconds. A transient signal of LA-ICP-OES can be obtained by generating a plot of emission intensity vs. time, in seconds.

As seen in figure 20 the selected signal to be integrated for this intensity determination is stable for the analytes of interest. The actual signal response attributed to the emission of the sample was determined by obtaining the difference between the recorded intensity during the time in 
which the ablated sample was being introduced and collected in the plasma, and the intensity prior to laser ablation. The intensity without laser ablation is referred to as a gas blank. It consists of the background emission present in the plasma that needs to be accounted for (subtracted from) the intensity of each analyte.

Reduction of the transient signal is accomplished using GeoPro laser ablation integration software (Cetac Technologies, Omaha, NE, USA). The software integrates windows of time before, during and after the laser ablation process that correspond to blank, analyte and carryover signal, respectively. The blank and carryover signals utilized $15 \mathrm{~s}$ time windows, while the analyte signal was integrated over a $20 \mathrm{~s}$ window of time. The window was selected so as not to include any of the initial coupling interactions between the laser and the sample. The window was kept constant for all experiments for each matrix.

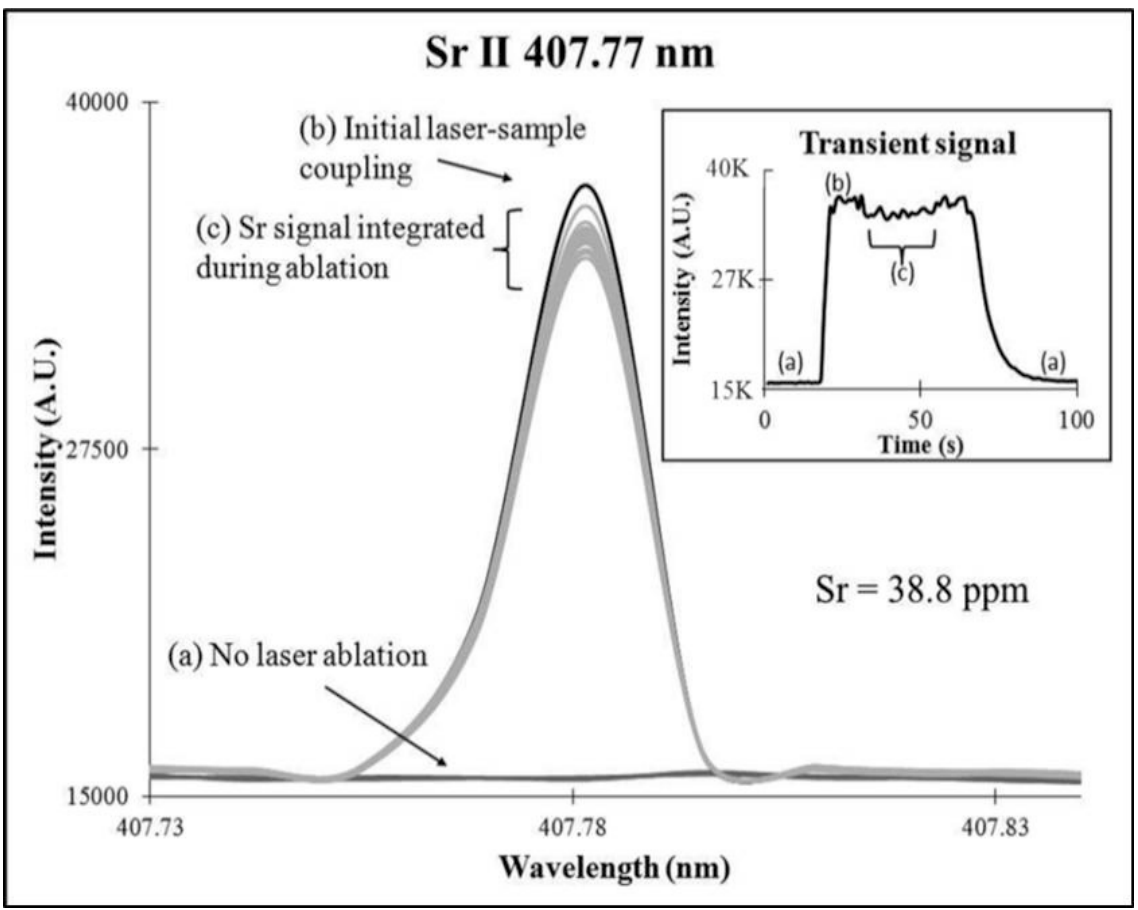

Figure 20: Transient signal integration for glass data interpretation. 


\subsubsection{Criteria for Evaluation of Studies}

The studies conducted within the scope of this paper were evaluated on the basis of several criteria. These criteria include comparisons of plasma conditions, analyte response and analytical performance. Plasma conditions were evaluated by comparing the ratio of ionic-to-atomic line intensity ratios, particularly $\mathrm{Mg}$ (II) $279.55 \mathrm{~nm} / \mathrm{Mg}$ (I) $285.21 \mathrm{~nm}$.

Additional evaluations were made on measurements of performance. The generation of a stable transient signal is directly influenced by the ability to generate and then efficiently transport ablated mass to the inductively coupled plasma (ICP). Once in the ICP, consistent plasma conditions promote reproducible emission, measured the relative standard deviation (\%RSD) of the emission signal. The overall analytical performance and assessment of the sensitivity of the method can be accomplished by using the LOD and determining the applicability and versatility of the method for analyzing simultaneously several analytes that vary in concentration and emission quality. It is this combination of criteria that was used to determine a potential optimal central channel gas composition flow for the technique.

\subsection{Results and Data Analysis}

\subsubsection{Comparison of Argon or Helium as Entire Central Channel Gas Composition}

Single gas composition flow rates of the central channel of the torch were determined using both the glass SRMs and the cotton standards. Because each type of material responds much differently to ablation, demonstrated in part by mass removed, $280 \mathrm{ng}^{95}$ and $9.9 \mu \mathrm{g}$, respectively, it was necessary to consider the responses of each matrix when evaluating total flow rates. Each gas was first considered as the sole component in the central channel in order to determine the optimal performance that could be achieved in this configuration. The values were obtained using the line scanning ablation mode employed for the daily performance evaluation, with the 
exception of a laser frequency of $5 \mathrm{~Hz}$ being used for cotton. Line ablation was chosen because of the longer length of time data needed for data collection. Spot ablation could only be conducted for $50 \mathrm{~s}$ and $60 \mathrm{~s}$ for cotton and glass, respectively. The amount of time was not long enough to axially scan the plasma via automation to determine optimal horizontal and vertical viewing coordinates. Line ablation ensured that the material reaching the torch was of similar morphology for the duration of sampling. Figure 21 depicts the maximum intensity recorded at each flow rate for Mg (I) $285.21 \mathrm{~nm}$ as well as Mn (II) $257.10 \mathrm{~nm}$ for both Ar and He environments using glass ablation as the example. Similar results were observed for the cotton standards.

Two different analytes were used to eliminate the potential for generalizations to be made on the basis of a single ionization mechanism given that $\mathrm{Mg}$ is highly susceptible to Penning ionization, particularly from Ar metastable species ${ }^{39,40,64,65}$. The influence of forward power was also considered. It was previously reported that optimal He conditions required a lower forward power $^{35}$, however this was not observed. The results coincide with LA-ICP-MS, corroborating that a higher forward power (i.e., $1500 \mathrm{~W}$ ) is needed for laser ablation sample introduction into an ICP, which further aides in reducing the size of the ablated mass, producing a more efficient ionization.

Although the NAZ has been defined as a broad region extending from 0-20 $\mathrm{mm}$ from the load $\operatorname{coil}^{97}$, the ICP properties of Ar and He vary, resulting in differently shaped NAZ zones especially when considering the response of $\mathrm{Mg}$. For $\mathrm{Mg}$ an entirely Ar ICP produces a distinct maximum, suggesting a more discrete normal analytical zone (NAZ). In addition, maximum intensities were recorded in the center both horizontally and vertically, for an Ar environment. While, although the He ICP produced two maxima for $\mathrm{Mg}$, the lower flow rates yielded off-axis maximum intensities, shifting sporadically $\pm 2 \mathrm{~mm}$ in both the horizontal and vertical directions when measurements were repeated. Normal variation was observed to be $\pm 0.5 \mathrm{~mm}$. Enhancement low in the plasma often occurs in response to an increase in collisional excitation ${ }^{35}$. Because a two- 
flow maximum is not observed for Mn (II) $257.10 \mathrm{~nm}$ this is most likely representative of Ar and Mg interactions ${ }^{39,40,65}$. The higher He flow rate maximum is corroborated by similar observations in the literature ${ }^{35}$.

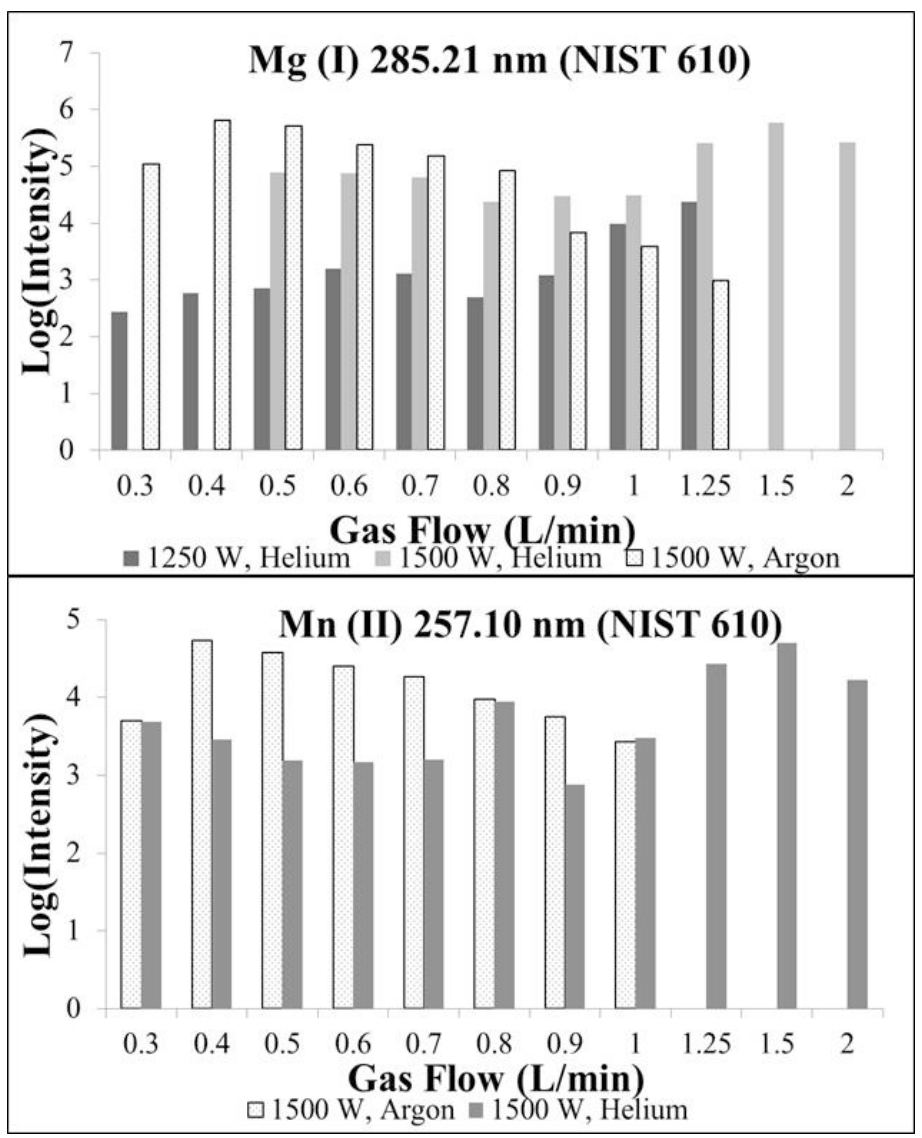

Figure 21: Maximum intensity observed with different gas compositions and flow rates in LA-ICPOES.

Although a maximum emission of similar intensity could be observed for each environment the generation of a robust plasma is still desired, and is reflected in overall performance and analytical capabilities of the method. The measurements for determining the robustness of a plasma for the two environments for each matrix is presented in table 13 . The values obtained are well above the conditions for establishing a robust operating environment in a continuous plasma source. 
Table 13: Robust plasma conditions for glass and cotton

\begin{tabular}{|l|c|c|c|c|}
\hline \multirow{2}{*}{ Central Channel Gas Flow } & \multicolumn{4}{|c|}{ Matrix } \\
\cline { 2 - 5 } & NIST610 & NIST612 & IAEA V-9 & NC2005 \\
\hline $1.25 \mathrm{~L} / \mathrm{min} \mathrm{He}$ & 19.4 & 15.5 & 16.3 & 19.4 \\
\hline $0.5 \mathrm{~L} / \mathrm{min} \operatorname{Ar}(213 \mathrm{~nm})$ & 12.1 & 10.7 & - & - \\
\hline $0.5 \mathrm{~L} / \mathrm{min} \operatorname{Ar}(266 \mathrm{~nm})$ & 11.3 & 10.1 & 15.4 & 15.4 \\
\hline
\end{tabular}

Because robustness can be achieved by two extremely different environments it then should also be considered which environment, or mixtures of environments should be applied to analytical LA-ICP-OES measurements for trace, minor and even bulk analysis.

\subsubsection{Assessment of He-Ar and Ar-He Mixtures for the Central Channel Gas}

\section{Composition}

In addition to single central channel gas composition, combinations of the two environments were conducted to determine if enhancement in the emission processes taking place in the ICP could be established, further improving the overall analytical performance of the technique. Both He-Ar and Ar-He mixtures were considered. The gas listed first in the mixture refers to the sweep gas, while the second gas corresponds to the composition of the nebulizer gas "T-ed" into the main analyte transport line. When selecting potential He-Ar or Ar-He mixtures for the evaluation of an optimal ratio for central channel gas composition instrumental limitations and analytical concerns were taken in to consideration. High central channel gas flow regardless of composition can influence the plasma impedance, resulting in frequent occurrences of the plasma being extinguished. This makes it difficult to change samples and can affect the ability to perform several analyses on a large number of samples. A gas flow exceeding approximately $1 \mathrm{~L} / \mathrm{min}$ resulted in the plasma being extinguished. In addition, as can be seen in figure 21, a flow of at least $0.3 \mathrm{~L} / \mathrm{min}$ is essential to even sweep the ablation cell to obtain some emission signal. Therefore the following stipulations were made when studying He-Ar and Ar-He ratios: initial 
gas flow through the ablation cell had to be at least $0.3 \mathrm{~L} / \mathrm{min}$ and the total gas flow following the addition of the second gas could not exceed $1 \mathrm{~L} / \mathrm{min}$.

The ratios were considered both by matching the flows and obtaining a He-Ar or Ar-He ratio of one as well as evaluating various mixtures. Because the maximum Ar intensity was obtained at $0.5 \mathrm{~L} / \mathrm{min}$, the Ar component of the mixture was not increased above this flow rate.

The mixing conditions included mixing the two gases before entering the ablation cell as well as combining the gas flows after the cell. The location in which the gases were mixed was also considered. Location was determined on the basis of the distance from the cell, either before or after. Although various tubing lengths were considered, the results for these studies are presented using only $1 \mathrm{~m}$ in length for the tubing connecting the ablation cell and the ICP torch since this tubing was found to be suitable for additional study parameters. The tubing length prior to the ablation cell was also kept constant at $1 \mathrm{~m}$. It was observed that the same Ar-He or He-Ar ratio yield similar results (within 5\%) if the above listed criteria were followed.

Figure 22 displays the transient signals for the Mg (I) $285.21 \mathrm{~nm}$ emission line in NC2005 obtained during laser ablation conducted using several different gas flow rates. Differences in emission intensity were observed for the different compositions. 


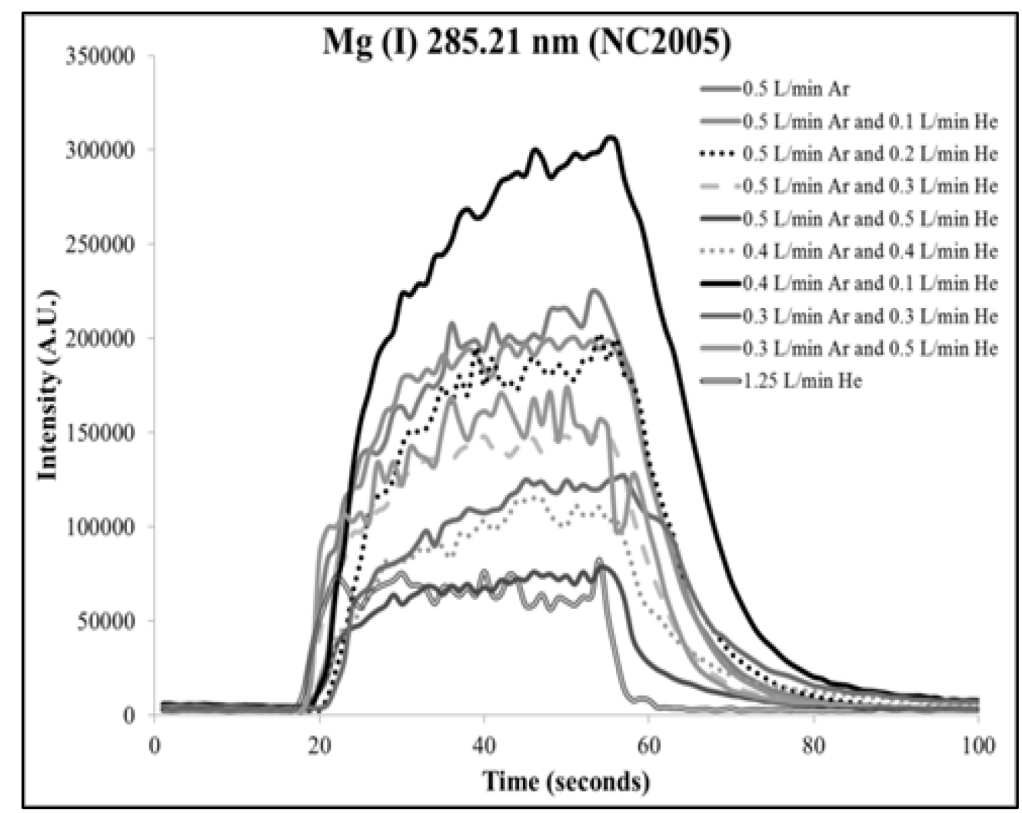

Figure 22: Transient signal for Mg in NC2005 using different gas flow rates and compositions.

Because $\mathrm{Mg}$ is present in higher concentration than other elements in cotton (360 ppm), and because of its well documented plasma interactions with Ar, results for a trace analyte, Mn (II) $257.10 \mathrm{~nm},(1.1 \mathrm{ppm})$ are shown in figure 23 . To simplify the observed trend, only the transient signals with the highest SNR were included in figure 23. The same gas mixtures resulted in the highest SNRs for Mg. While the effect of gas composition appears to be more dramatic for analytes in higher concentration $(\mathrm{Mg})$, trace analyte signal is not significantly affected with change in composition of the central gas channel. Since the concern of method development is typically to accomplish sensitive analysis of trace analytes, the comparable Mn signal suggests that similar performance can be obtained for analytes of different concentration in a particular matrix that may employ different ionization mechanisms. 


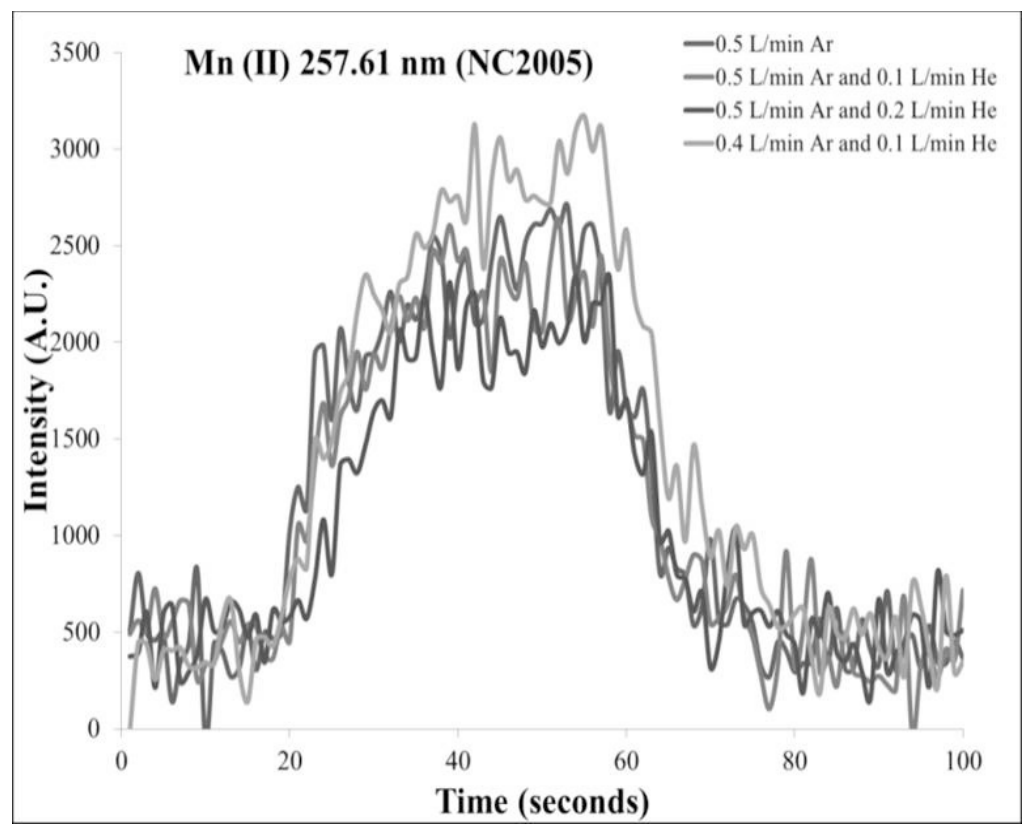

Figure 23: Transient signal for Mn in NC2005 using different gas flow rates and compositions.

The independence from route of ionization and even sensitivity ( $\mathrm{Mn}$ is present at $\sim 1 \mathrm{ppm}$ ) infers that analytical measurements can be performed with any of these combinations presented in figure 23, without sacrificing sensitivity of low concentration elements when a single matrix is considered. To assess the validity of this concept interpretation of the transient signals of glass constituents was also performed.

The performance of glass for different Ar-He combinations is displayed using the resulting transient signals for SRM NIST 1831 in figure 24. The results for two analytes of high (Mg) and low (Ti) concentration to address the analytical performance that could be obtained for analytes of different concentration in glass. The number of Ar-He gas combinations was limited in figure 24 in order to present the trends in emission clearly. Again, the same combinations produced the highest SNR in glass as in cotton. The stability of the transient signals from figures 22-24 establish that precise measurements (\%RSDs $>1-8 \%$ ) can be achieved with these low flow configurations. Because very little difference in signal was observed for Mn and Ti emission implies that the emission of these trace analytes is not influenced by gas composition. Since the 
sensitivity of low concentration analytes in trace analysis is crucial it can be stated that measurements can be made with any of the four central channel gas mixture present. However, it can also be concluded that because not all analytes displayed a change in the presence of $\mathrm{He}$ suggests that He is not as critical to the analytical performance of LA-ICP-OES as it is in LAICP-MS analysis.

To further demonstrate that the results are not only independent of matrix, the glass SRMS were also analyzed using the same conditions on the LSX-500 laser ablation unit. Relative intensities are presented in table 14 of some analytes for SRM NIST 1831. The signals are normalized to a silicon ( $\mathrm{Si}$ (I) $221.66 \mathrm{~nm}$ ) emission line used as an IS to account for any day-to-day variation that could exaggerate observed trends. The closer the relative intensity is to one, the more similar the results. Intensities were compared to that which has been obtained with $0.5 \mathrm{~L} / \mathrm{min}$ Ar.

Table 14: Comparison of SRM NIST 1831 ablation using $213 \mathrm{~nm}$ and $266 \mathrm{~nm}$ wavelength lasers by means of relative intensity

\begin{tabular}{|l|c|c|}
\hline \multicolumn{1}{|c|}{ Analyte } & Relative Intensity & Standard Deviation \\
\hline $\mathrm{Al}$ (I) $396.15 \mathrm{~nm}$ & 0.82 & 0.06 \\
\hline $\mathrm{Ba}$ (II) $455.40 \mathrm{~nm}$ & 0.88 & 0.02 \\
\hline $\mathrm{Ca}$ (II) $315.88 \mathrm{~nm}$ & 0.86 & 0.02 \\
\hline $\mathrm{Fe}$ (II) $238.20 \mathrm{~nm}$ & 0.90 & 0.008 \\
\hline $\mathrm{Li}$ (I) $670.78 \mathrm{~nm}$ & 1.1 & 0.008 \\
\hline $\mathrm{Mg}$ (I) $285.21 \mathrm{~nm}$ & 0.93 & 0.007 \\
\hline $\mathrm{Sr}$ (II) $407.77 \mathrm{~nm}$ & 0.92 & 0.006 \\
\hline $\mathrm{Ti}$ (II) $368.51 \mathrm{~nm}$ & 0.95 & 0.02 \\
\hline $\mathrm{Zr}$ (II) $343.82 \mathrm{~nm}$ & 0.91 & 0.01 \\
\hline
\end{tabular}



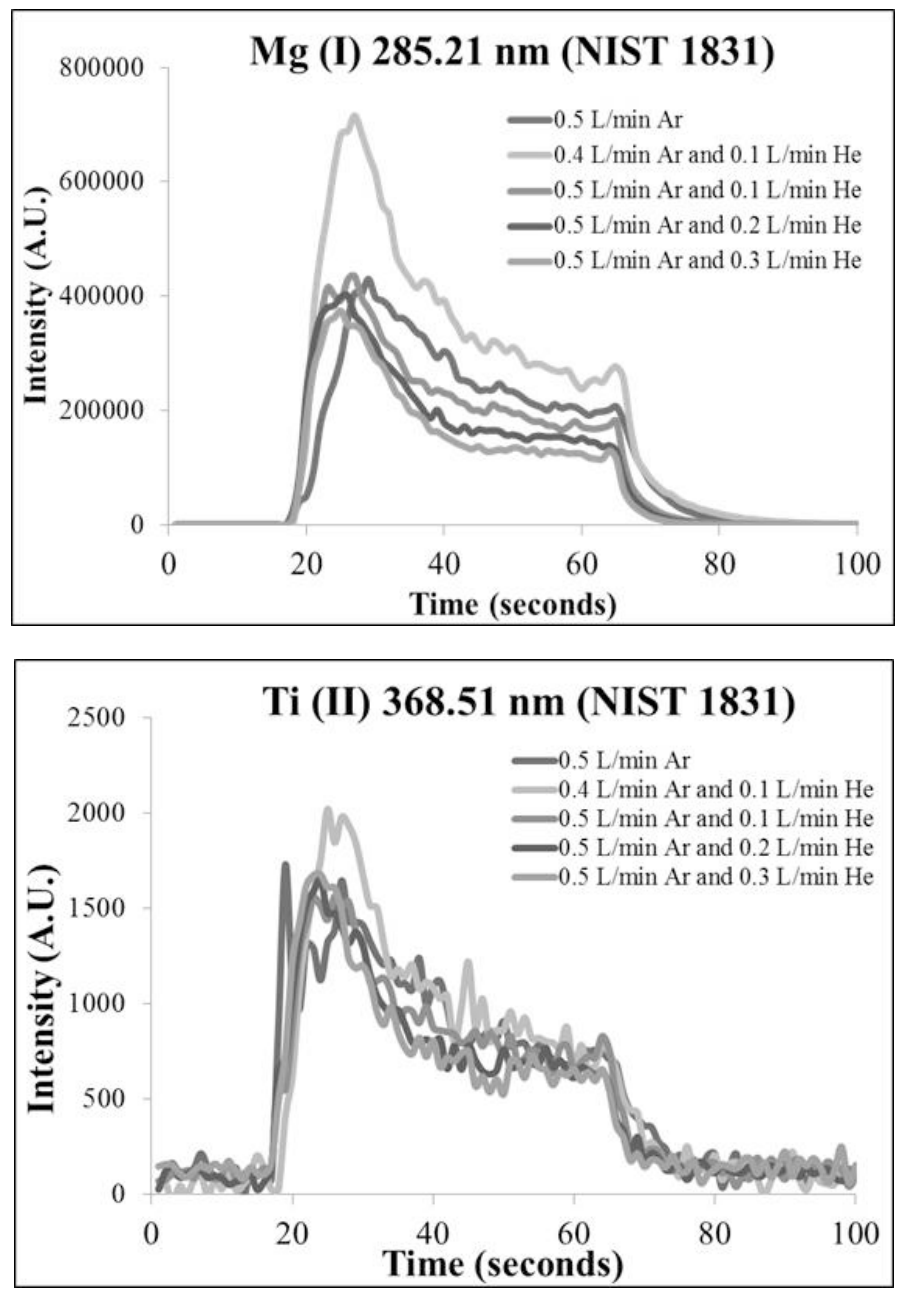

Figure 24: Transient signals in glass for elements of varying concentration.

The similarity in responses confirms that the optimal gas flow configuration is the same regardless of matrix or laser ablation setup. In addition, the differences in ablation cell volume did not affect outcomes in emission, suggesting that even at low flow rates the cell is still adequately swept. These findings imply uniformity in method performance regardless of matrix.

However, the shape of the transient signal varies for each matrix. Initial laser-sample interactions, i.e., factors in coupling, were more prominent in glass and varied with gas composition. The first laser-sample interactions produce larger particles which translate to a higher signal, although unstable in stability and duration. As the quality of the laser-sample interactions improve, smaller, 
more uniform particles are ejected from the material and are subject to ionization. Uniformity in the transported mass results in a stable transient signal.

It is interesting to note that as the He content increased in the central channel the extent of the intense initial emission decreases; this trend is overly apparent when the environment is entirely He, showing no initial spike in the emission signal. Helium possesses thermal properties that prevent aggregation of the ablated material, reducing the presence of the initial laser coupling emission $^{72}$. The property has been thoroughly explored in relation to LA-ICP-MS and has been utilized to improve the transient signal ${ }^{72}$.

The lower the total central channel gas flow, the higher the emission signal. Lower overall flow rates for ICP-OES have been shown to prolong the residence time in the plasma, increasing the opportunity for detection of emission ${ }^{81}$. Residence time refers to the lifetime of a species in the

plasma $^{81}$. This holds true and is demonstrated in the results obtained using LA-ICP-OES for Ar and He-Ar mixtures. Although the opposite is observed for an entirely He flow. It is proposed that the lower density of He requires a higher flow rate to increase the pressure through the injector. The pressure results in slower movement through the plasma, also producing a longer residence time. Longer residence time and the production of smaller particles is not able to overcome the higher IP of this gas.

\subsubsection{Influence of Central Channel Gas Composition on Particle Size Distribution}

The influence of particle size distributions as a result of central channel gas composition was addressed using a particle filter device built in our laboratory. Magnesium, Mn and Sr transient signals for NC2005 using $0.5 \mathrm{~L} / \mathrm{min}$ Ar as the sweep gas and $0.1 \mathrm{~L} / \mathrm{min}$ He as additional makeup gas are shown in figures 25-27. The use of He, even at the low flow has an influence on particle size, as demonstrated by the increase in intensity from the filter being located before the 
introduction of He to after the Ar-He are mixed in the central channel. This was easily observed for the high concentration of $\mathrm{Mg}$ and somewhat so with $\mathrm{Mn}$. To support that the reduced particle size was indeed acquired as a result of the presence of He the $\mathrm{Sr}$ transient signal was also included.

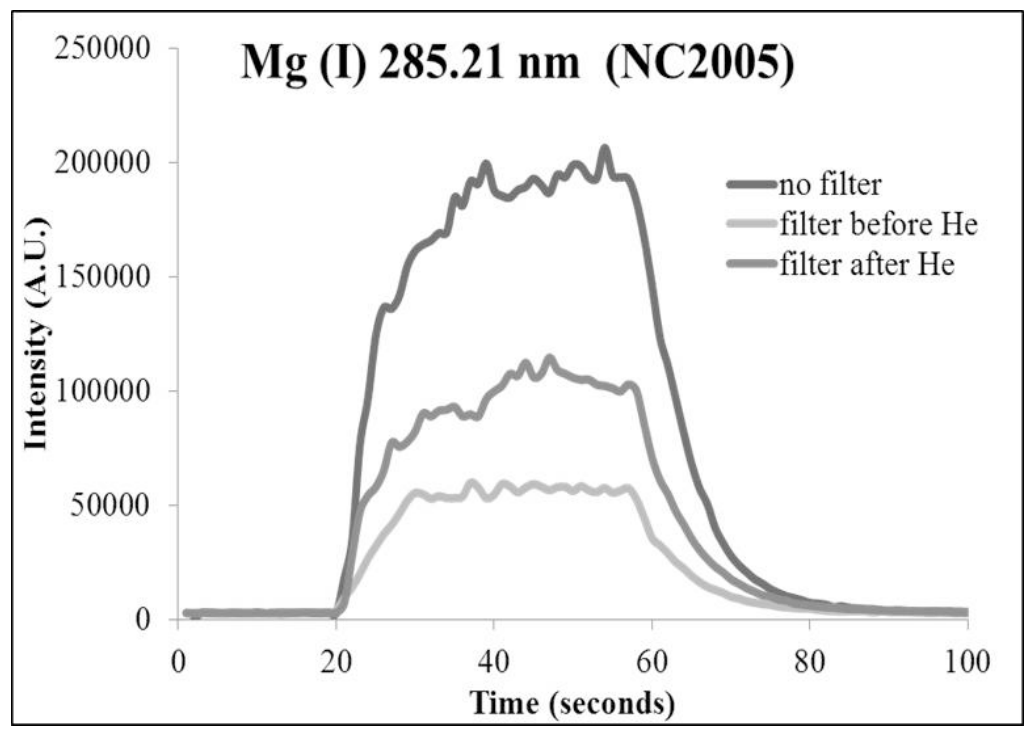

Figure 25: NC2005 Mg transient signals obtained with the filter device.

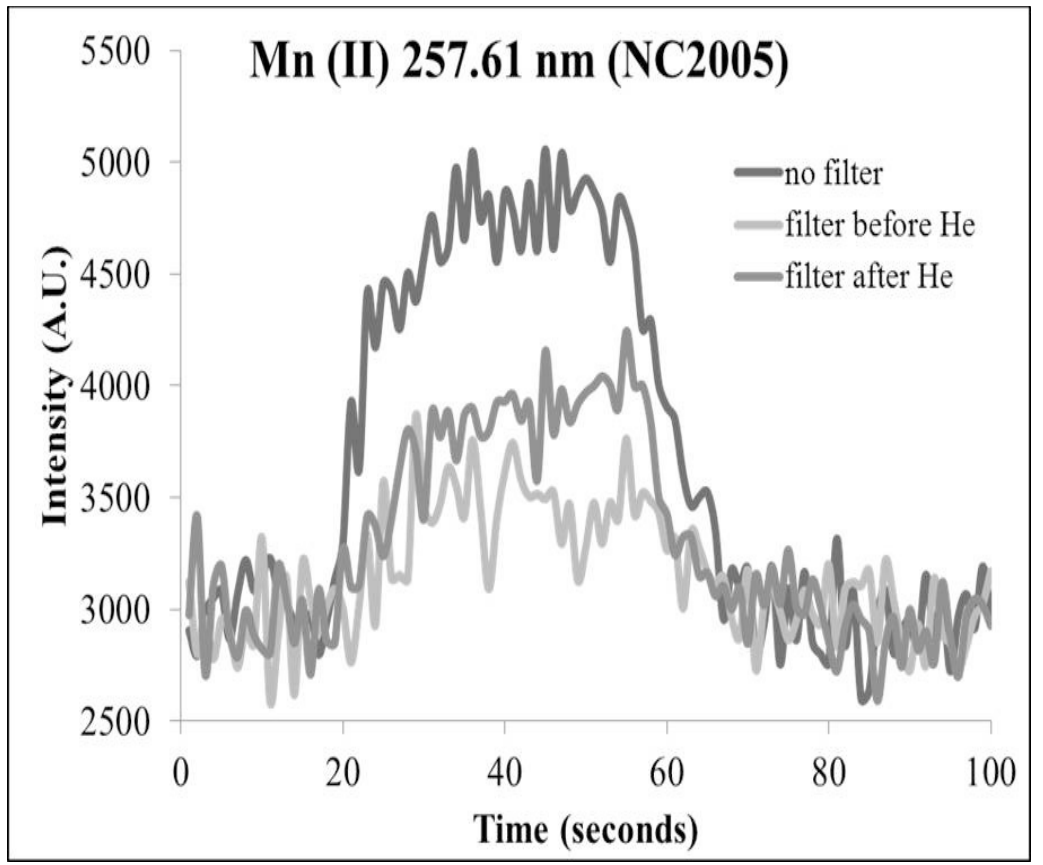

Figure 26: NC2005 Mn transient signals obtained with the filter device. 


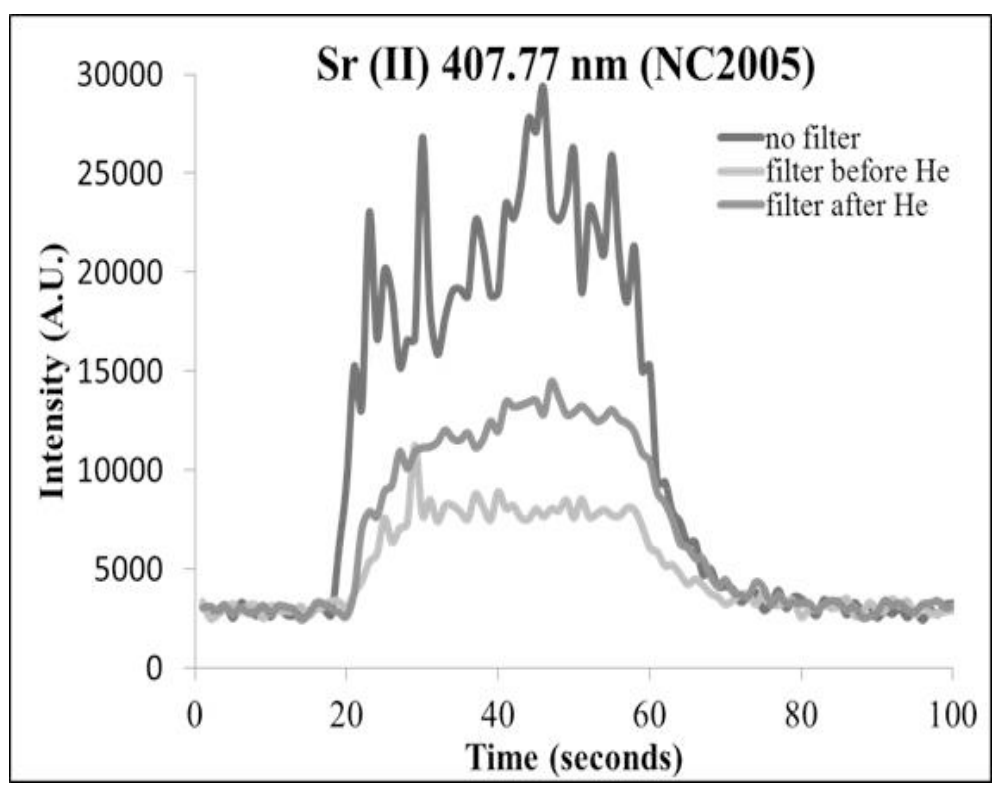

Figure 27: NC2005 Sr transient signal obtained with the filter device.

Because the filter limits the size of the particles that can reach the ICP the increase in signal following the addition of $\mathrm{He}$ then it can be concluded that even in small amounts, the thermal conductivity properties of this gas are apparent. To ensure that the performance of any of the gas mixtures initially presented in figure 22 was not the result of changes in the ratio of the gas mixture, which may influence residence time in the ICP, the data were compared in turns of density of the gas mixture. Because no discernible trend could be determined, it is also assumed that the change in density following the addition of He was not responsible for the increase in emission.

The utilization of small amounts of $\mathrm{He}, 0.1 \mathrm{~L} / \mathrm{min}$ with $0.4 \mathrm{~L} / \mathrm{min}$ or $0.5 \mathrm{~L} / \mathrm{min}$ Ar may be sufficient to prevent aggregation of particles, leading to a more thorough ionization in the plasma. It is not likely that $\mathrm{He}$ is able to aid in ionization in the plasma, mostly because of its much higher IP than Ar. The high energy He would prevent ionization mechanisms that rely on energy transfers utilizing a lower energy species and analytes. Energy transfers typically occur between 
two constituents of similar energy. This directly influences the emission processes and inhibits the ability to make analytical measurements with a high degree of sensitivity. Helium can only provide energy transfers for halogens.

The ability to ionize the gas in the central channel of the ICP torch is crucial to achieving sufficient ionization and excitation of a wide range of analytes. Argon and Ar metastable species are more conducive to interactions related to Penning ionization and other energy transfers than $\mathrm{He}^{35}$ is capable of yielding.

\subsubsection{Effect of Transport Tubing Length}

The length of transport tubing was varied in order to determine the effect on the signal, in both the intensity and stability. The tubing length was varied between two extremes, $0.5 \mathrm{~m}-2 \mathrm{~m}$. On the basis of the results of the He-Ar and Ar-He studies the effect of tubing length was studied using $0.5 \mathrm{~L} / \mathrm{min}$ Ar. Because the total flow rates employed in this work were much lower than those reported for LA-ICP-MS it was a concern that an increase in the tubing length could result in a diffusion or slower transport of ablated mass to the ICP torch. To account for the possibility of variable time to return to baseline, all replicates in this study were conducted using 150 readings per replicate in order for the entire transient signal to be used for comparisons. As the tubing length was increased under the same central gas compositions and laser parameters, no change was observed for the shape and stability of the transient signal, figure 28 , or the analytical performance. Lower flow rates resulted in negligible, if any, diffusion in the mass transport to the ICP torch. Lower overall flow rates did yield higher emission signals than higher gas flow mixtures. 


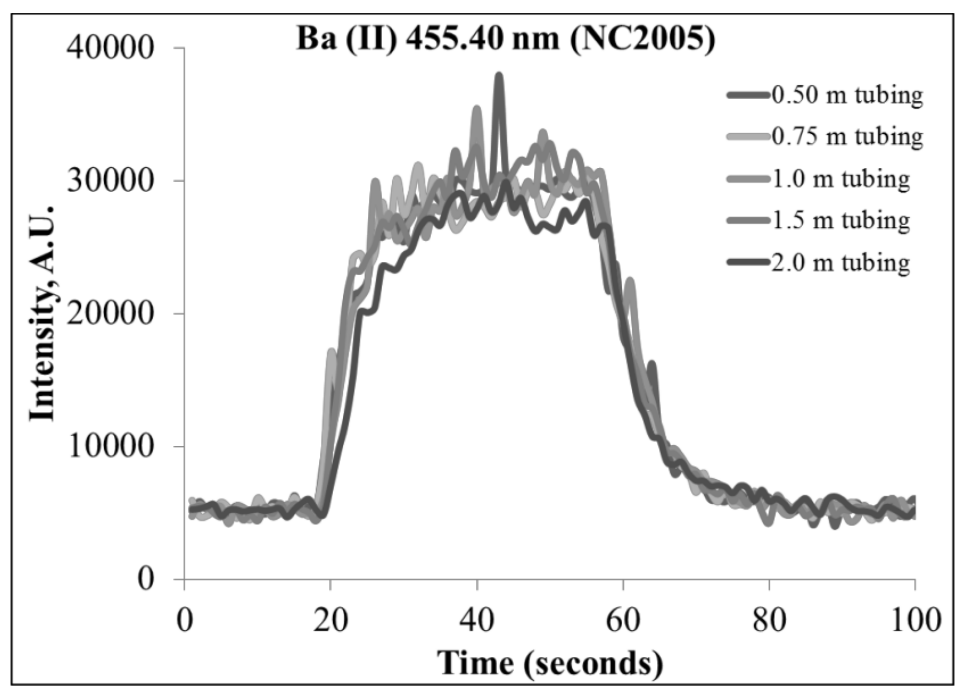

Figure 28: Effect of tubing length on transient signal.

If varying gas compositions are considered at a single tubing length, lower overall flows resulted in higher signals. The tubing length used in all other experiments was $1 \mathrm{~m}$ in length from the ablation cell to the torch.

\subsubsection{Influence of Central Channel Gas Composition on Analytical Performance}

The LODs were calculated for the three He-Ar parameters that resulted in the highest SNR with the most stable transient signals for the cotton matrix using the LSX-500 unit, and the glass matrix using the UP-213 unit. Different laser ablation units were chosen to determine the optimal analytical performance because glass has been shown to achieve better laser-sample interactions when subjected to lower wavelength irradiation ${ }^{98}$. Table 15 depicts the sensitivity that could be achieved with each of the three gas combinations that resulted in the highest SNR for the two matrices. 
Table 15: Analytical performance for different gas configurations in LA-ICP-OES

\begin{tabular}{|l|c|c|c|c|c|c|c|c|}
\hline \multirow{2}{*}{ Gas Composition } & \multicolumn{3}{|c|}{ Cotton LODs (in ppm) } & \multicolumn{3}{c|}{ Glass LODs (in ppm) } \\
\cline { 2 - 9 } & $\mathbf{M g}$ & $\mathbf{S r}$ & $\mathbf{F e}$ & $\mathbf{M n}$ & $\mathbf{M g}$ & $\mathbf{S r}$ & $\mathbf{F e}$ & $\mathbf{T i}$ \\
\hline $0.5 \mathrm{~L} / \mathrm{min} \mathrm{Ar}$ & 0.20 & 0.090 & 0.20 & 0.11 & 8.1 & 0.20 & 13.1 & 6.9 \\
\hline $\begin{array}{l}0.4 \mathrm{~L} / \mathrm{min} \mathrm{Ar} \\
0.1 \mathrm{~L} / \mathrm{min} \mathrm{He}\end{array}$ & 0.20 & 0.10 & 0.51 & 0.17 & 5.3 & 0.50 & 13.7 & 7.3 \\
\hline $\begin{array}{l}0.5 \mathrm{~L} / \mathrm{min} \mathrm{Ar} \\
0.1 \mathrm{~L} / \mathrm{min} \mathrm{He}\end{array}$ & 0.18 & 0.080 & 0.90 & 0.15 & 9.21 & 0.43 & 14.5 & 7.0 \\
\hline
\end{tabular}

Emission lines used are Mg (I) $285.21 \mathrm{~nm}$, Sr (II) $407.77 \mathrm{~nm}$, Fe (II) $238.20 \mathrm{~nm}$, Mn (II) $257.10 \mathrm{~nm}$ and Fe (II) $238.20 \mathrm{~nm}$ (glass) and Fe (I) $259.93 \mathrm{~nm}$ (cotton).

When considering the element menu, or at least several elements of varying concentrations, little difference in LODs is observed for the three gas mixtures. The LODs have been calculated using the approach outlined in equations 16 and 17 . The higher LODs for some analytes can be directly related to the concentration in which the analyte is present $(>10 \mathrm{~K} \mathrm{ppm})$. As a result of the high concentration, less sensitive emission lines have been selected to avoid saturation of the detector. This negatively impacts the LODs of these particular analytes.

In addition, the LODs obtained are suitable in both instances for conducting trace analysis. Suppression from $\mathrm{Ca}$ in glass analysis appears not to have negatively affected the ability to achieve LODs in the low ppm range. The contribution of Ca could be considered nonexistent because it was, although high, relatively constant (5-8\% concentration). This reported matrix effect could be significant if the Ca content varied more drastically.

The overall goal of the analytical method - whether it is trace analysis or determining the presence of minor constituents - should be understood when operating conditions are selected. When conducting analytical analysis for several constituents the versatility of the method must be considered. The ability to provide sensitive measurements is critical. Because comparable quantitative capabilities can be achieved for most, if not all analytes for cotton and glass, Ar has 
been selected to comprise the entire central channel gas composition. This alleviates some complexity encountered when using external gas lines and gas flow regulators.

\subsection{Discussion and Conclusions}

The results of these studies demonstrate that there are certain LA-ICP-OES operating conditions that can be generalized for different matrices and different laser ablation cells. Because these results were obtained using two matrices that differed in physical and chemical characteristics, which is reflected in the ablation properties were compared, this suggests that these conclusions can be generalized to different matrices - increasing the significance of results.

In general, the lower the flow rate (i.e., $0.5 \mathrm{~L} / \mathrm{min}$ ) of the central channel of the ICP will result in higher emission intensities than a higher flow rate (i.e., $1 \mathrm{~L} / \mathrm{min}$ ) due to the increase in residence time in the plasma and the opportunity for numerous energy transfers to take place. The application of He gas as the sweep gas or a component of the central channel gas for LA-ICPOES, while smaller particles were observed, demonstrated by the decrease in initial laser coupling emission and the indirect evaluation of particle size measured with the filter device, did not result in a significant improvement in analytical sensitivity or performance. This suggests that particle size may not be as crucial as in LA-ICP-MS because LA-ICP-OES was able to accomplish trace analysis regardless of He gas content. Argon provides a more conducive environment for LA-ICP-OES analysis.

Matrix effects determined by low second IP elements, like Ca, was not considered to be a threat to conducting the trace analysis. Especially demonstrated when considering glass analysis, in which trace elements were present in the low ppm range and Ca was present in the glass standards in concentrations ranging from $\sim 58 \mathrm{~K}-85 \mathrm{~K}$ ppm. 
At the low flow rates recommended in this research, tubing length was not critical. Also, the use of Ar as the only gas for the sweep and nebulizer composition allows for the simplification of analytical setup of the instrument. This is an attractive feature for implementation in laboratories for the development of additional applications.

\section{PART 2: FORENSIC APPLICATIONS}

\subsection{FORENSIC GLASS ANALYSIS BY LA-ICP-OES}

\subsection{Overview}

The elemental analysis of glass evidence has been established as a powerful discrimination tool for forensic analysts. The use of LA-ICP-OES has been compared to LA-ICP-MS and energy dispersive micro X-ray fluorescence spectroscopy ( $\mu \mathrm{XRF} / \mathrm{EDS})$ as competing instrumentation for the elemental analysis of glass. The development of a method for the forensic analysis of glass coupling laser ablation to ICP-OES is presented for the first time herein. This technique has demonstrated comparable analytical performance to LA-ICP-MS when comparing overlapping element menus. The analytical figures of merit are presented and compared to that which can be achieved using LA-ICP-MS in order to address the applicability of this technique for the elemental analysis of glass. In addition, a set of vehicle glass samples was used to assess the discrimination power of the LA-ICP-OES method in comparison to other elemental analysis techniques. This sample set consisted of several vehicle glass samples that originated from the same source (inside and outside windshield panes) and several glass samples that originated from different vehicles. Different match criteria were used and compared to determine the potential for Type I and Type II errors. Results presented in this chapter suggest that a broader match criteria is more applicable to the forensic comparison of glass analysis because it can reduce the effect that 
micro-heterogeneity inherent in the glass fragments and a less than ideal sampling strategy can have on the interpretation of the results.

\subsection{Introduction}

\subsubsection{Glass Matrix}

Glass making is a longstanding art, having been made - either by hand or by manufacturing techniques - for thousands of years ${ }^{99}$. This is not surprising since the main components of glass sand, soda ash and limestone - are inexpensive and have been readily available to humans for quite some time.

Glass is defined according to the American Society for Testing and Materials (ASTM) as “...an inorganic product of fusion that has been cooled to a rigid condition without crystallization..." Often times glass is not considered a solid, but rather it exists between solid and liquid states in what is called the "glassy state" 101 . A controlled cooling process allows glass to form a durable crystalline-like substance, similar to a solid, which also has the internal disorder of a liquid. The properties of a "glassy state" fall between those of a solid or liquid, in most instances yielding the ideal properties from each state. For example, glass has a broad range for melting, rather than a finite point. It is these physical properties of glass that make it a unique matrix, with an extensive variety of uses spanning from structural (architectural windows) to dishware and ornamental glassware.

It is these numerous uses of glass that result in the common encountering of this matrix at several different types of crime scenes during forensic investigations. Because of its abundance, methods of characterizing the glass (physical, chemical optical properties) are used to characterize the known and question glass fragments. Since this research focuses on the application of elemental analysis as a means to associate or discriminate glass samples the remainder of this section will 
address and describe the manufacturing process and the role of minor and trace elemental constituents play in the intentional or unintentional introduction during glass production.

Glass is manufactured on a large scale in a chain of industrial furnaces capable of achieving extremely high temperatures. The most abundantly produced glass is manufactured using the "float glass" method, accounting for $90 \%$ of manufactured glass ${ }^{102}$. Particularly because of its use in architectural and vehicle windows, this glass makes it a commonly encountered piece of evidence in forensic investigations. Float glass, also known as sheet glass, refers to any glass that is made in large, continuous flat panels. The method was developed by the Pilkington brothers in England during the $1950 \mathrm{~s}^{103}$. The Pilkington brothers revolutionized this type of glass making by improving the process to yield more evenly distributed molten glass so that the final glass product is flatter with fewer surface imperfections. They accomplished this by pulling a continuous ribbon of hot glass across the surface of a molten metal ${ }^{103}$. Because the glass never has the opportunity to come in contact with a solid the hot glass "floats" on top of the metal, which produces a uniform glass surface. The molten metal employed in the float glass process is tin. Tin is ideal for this application because it does not mix with the molten glass, thus keeping an imaginary boundary between the two substances.

In addition to the application of a molten metal, the float glass process also employs several furnaces in which the temperatures are carefully controlled. It is during these stages that annealing occurs. Annealing is a critical step in glass production because this results in the generation of a durable glass state, capable of withstanding a significant amount of applied stress. The process of annealing involves slowly cooling the glass in such a manner that the temperature is uniform throughout; the glass is slowly cooled through the critical temperature range ${ }^{101}$, relieving internal stress and strengthening the chemical bonds within. Annealed glass is much stronger than glass that has not been subjected to such a procedure. 
The Pilkington brother's float glass method is advantageous in that the glass can be made continuously as long as the raw materials are available. This modification to glass making has increased the productivity of this industry, compensating for the constant demand of this product for its vast uses. In addition, the improvements in float glass production yield a more homogeneous matrix than what was produced prior to the 1950s. Although heterogeneity on a micro-scale persists, the improved quality of this good does translate to a more uniform product.

Certain types of float glass require additional finishing processes. The finishing processes are used to cater the glass for end use. Relevant finishing processes include thermal finishing and tempering ${ }^{103}$. Thermal finishing is used to bend two sheets of glass together in a bending furnace, producing the curvature needed for windshields. A windshield is comprised of two sheets of glass laminated together with a plastic layer in between them. The plastic is often tinted to provide a ultraviolet filter ${ }^{103}$. On the other hand, tempering is used to complete the manufacturing of side and rear automobile windows. Tempering can be considered a controlled disannealing ${ }^{103}$; the glass is heated to almost the point of softening and then rapidly cooled with blasts of cold air ${ }^{103}$. Whereas annealing removes stress from the glass, tempering introduces stress. Tempered glass contains a significant amount of internal tension that is compacted from both surfaces of the glass. When the internal stress is disturbed by an outside force the glass breaks. While this is expected of glass, the manner in which tempered glass breaks is different from sheet glass. Tempered glass breaks into several small blunt cube-shaped fragments. When an overwhelming amount of stress is applied to windshield glass it breaks into sharp shard of glass. It is finishing processes like these that, in some instances allow the shape of fragment to be used to determine the type of float glass.

A significant amount of material is needed to produce the large batches of glass. Each furnace can hold up to 20 tons of raw materials at any given point in time ${ }^{103}$. Besides the three raw 
constituents listed earlier, there are several other additional components that comprise a batch of glass. It is what goes into making each batch that is used by forensic analysts to differentiate glass fragments on a chemical level. For example, cullet is added to every batch of glass. Cullet refers to the scrap or excess glass material recycled from previous batches ${ }^{103}$. The scrap can contain different proportions of additives than the current batch, adding a degree of heterogeneity between batches. There are several other agents added to the glass for specific purposes or to achieve optimal properties. Categories and common examples of these additives and others can be found in table 16. Although not all-inclusive, this list does provide insight as to the differences in elemental composition that can be obtained during glass production. There are an infinite number of possible combinations of these additives, and other trace elements (some of which may be unintentional) in glass. Unintentional trace elements introduced into the glass manufacturing process come from the slow corrosion and leaching of the furnaces. The variation in elemental profile is exploited by forensic analysts and has been reported throughout the literature for the application of glass discrimination for forensic purposes ${ }^{96}$. 
Table 16: Common glass constituents ${ }^{103,104}$

\begin{tabular}{|c|c|c|}
\hline Raw Material & Chemical Composition & Rationale \\
\hline Sand & $\mathrm{SiO}_{2}$ & $\begin{array}{l}\text { Network former (major } \\
\text { ingredient) }\end{array}$ \\
\hline Soda ash & $\mathrm{Na}_{2} \mathrm{CO}_{3}$ & Flux agent (fusion agent) \\
\hline Limestone & $\mathrm{CaCO}_{3}$ & $\begin{array}{c}\text { Reduces melting temperature and } \\
\text { viscosity of } \mathrm{SiO}_{2}\end{array}$ \\
\hline Salt cake & $\mathrm{Na}_{2} \mathrm{SO}_{4}$ & $\begin{array}{c}\text { Reduces melting temperature and } \\
\text { viscosity of } \mathrm{SiO}_{2}\end{array}$ \\
\hline Dolomite & $\mathrm{CaCO}_{3} \cdot \mathrm{MgCO}_{3}$ & $\begin{array}{c}\text { Reduces melting temperature and } \\
\text { viscosity of } \mathrm{SiO}_{2}\end{array}$ \\
\hline Feldspar & $\mathrm{K}_{2}\left(\mathrm{Na}_{2}\right) \mathrm{O} \cdot \mathrm{Al}_{2} \mathrm{O}_{3} \cdot 6 \mathrm{SiO}_{2}$ & Durability, intermediate former \\
\hline Litharge & $\mathrm{PbO}$ & Increased refractivity \\
\hline Alkaline oxides & $\mathrm{Na}_{2} \mathrm{O}, \mathrm{K}_{2} \mathrm{O}$ or $\mathrm{Li}_{2} \mathrm{O}$ & $\begin{array}{c}\text { Decreases potential of } \\
\text { devitrification, reduces melting } \\
\text { point }\end{array}$ \\
\hline Additional oxides & $\mathrm{CaO}, \mathrm{MgO} . \mathrm{ZnO}$ or $\mathrm{BaO}$ & $\begin{array}{c}\text { Additional oxides used to } \\
\text { decrease devitrification, reduces } \\
\text { melting point }\end{array}$ \\
\hline Various sources & $\begin{array}{c}\mathrm{Fe}_{2} \mathrm{O}_{3} \text { (brown, blue), } \mathrm{Cr} \text { (green), } \\
\mathrm{Mn} \text { (purple), Ce (yellow), } \mathrm{Au} \\
\text { (Red) }\end{array}$ & Colorants \\
\hline Various sources & $\mathrm{ZnO}, \mathrm{As}_{2} \mathrm{O}_{3}, \mathrm{MnO}_{2}, \mathrm{CoO}$ & Decolorizer, often added to cullet \\
\hline Various sources & $\mathrm{As}_{2} \mathrm{O}_{3}, \mathrm{CaSO}_{4}$ & Refining agents \\
\hline
\end{tabular}

\subsubsection{Forensic Analysis of Glass}

Forensic analysis revolves around the capability to establish associations between evidence recovered at a crime scene to a given source on the basis of the trace analysis of materials. The significance of these associations depends on the likelihood that the proposed relationship is case specific, common or uncommon. The less common the association the more significant it becomes, the more value it has to a forensic investigation. This concept has motivated researchers and practitioners to develop analytical approaches as well as improve the sensitivity of existing techniques for the examination of trace evidence. A model matrix to evaluate the applicability of a technique that is commonly encountered in forensic investigations is glass, and in particular vehicle glass. 
Associations determined by glass evidence can be established from a physical match of fragments or from the chemical and physical properties of the glass. The presence of a fracture match, or physical match between the known and unknown fragments can be used to make a definitive association. However, this association is very rare. Typically the standard approach to glass analysis is to first evaluate the physical characteristics - color, type, thickness, unique surface characteristics and refractive index (RI). If once the comparisons of physical characteristics are exhausted and no significant difference can be found between the known and unknown fragment(s) chemical analysis is then used to confirm and strengthen associations on the basis of the physical examination. Chemical comparisons are required for the analysis of glass because the use of physical and optical properties for the discrimination between different sources of glass has become less useful since manufacturing processes are optimized to achieve a narrow range of refractive indices and density values for optimum product quality ${ }^{105}$. The narrow ranges result in a high probability that similar, if not indistinguishable refractive indices can be observed even if the glass samples are from different sources ${ }^{105}$.

Chemical composition or, more specifically, the quantitative analysis of the trace elements in glass has been measured and compared as a means to distinguish (or associate) glass samples submitted as evidence ${ }^{106}$. There have been several instances in which the use of elemental analysis in addition to RI has been able to provide more information than RI alone. Elemental analysis is capable of enhancing the informing power of glass. The potential of elemental analysis to be used in place of or accompanying RI measurements has been demonstrated using a fiveelement menu to improve the discrimination power of glass from the same sub type ${ }^{106}$. While elemental analysis comparisons is capable of enhancing the discrimination between glass sources over RI, the application of RI still serves as a speedy, simple screening tool to quickly exclude associations is still a recommended approach to include in the forensic examination of glass. 
Several analytical methods including scanning electron microscopy with energy dispersive X-ray spectroscopy (SEM-EDS), XRF ${ }^{107}$, solution ${ }^{108}$ and laser ablation ${ }^{109,110}$ sample introduction for ICP-MS, and solution-based ICP-OES or ICP-AES ${ }^{107,111}$ have been implemented for the elemental analysis of glass. All of the techniques, though capable of distinguishing glass originating from different sources, or glass originating from different times within a short production interval within the same manufacturing plant ${ }^{112}$ each suffers from specific limitations that affect the implementation of these methods in forensic laboratories.

Both SEM-EDS and XRF have similar operating principles, using high energy electrons or Xrays to knock off outer shell electrons, which is then followed by relaxation to an unoccupied position, resulting in the emission of characteristic X-rays ${ }^{104}$. As previously mentioned, trace elements are often utilized to distinguish glass samples; however these techniques often lack the sensitivity to see this variation. Element ratios including $\mathrm{Ca} / \mathrm{Mg}, \mathrm{Na} / \mathrm{Al}, \mathrm{Mg} / \mathrm{Al}, \mathrm{Ca} / \mathrm{K}$ and $\mathrm{Ca} / \mathrm{Na}^{113}$ and $\mathrm{Ca} / \mathrm{Mg}$ and $\mathrm{Ca} / \mathrm{Fe}^{114}$ have been utilized to distinguish glasses by SEM-EDS, and SEM-EDS and XRF, respectively. The element menus of these technique often consist of elements that are observed in high concentration in float glass, with few instances of trace constituents like $\mathrm{Sr}$, Ti and $\mathrm{Zr}$ being reported in the literature ${ }^{115,116}$. While SEM-EDS and XRF may suffer from the inability to detect trace analytes both are capable of determining glass type ${ }^{107,114,115}$, and have even reported discrimination powers of $98 \%{ }^{115,117}$ for distinguishing glass samples within one or between two types of glass. However, the inability to detect trace elements that are critical for characterizing differences within a glass type and other drawbacks like the need for flat, relatively non irregular shaped fragments that even still usually require resin embedding do not make SEM-EDS and XRF suitable techniques for identifying differences in forensic glasses. 
Other techniques used for the elemental analysis of glass include ICP techniques. Both ICP-MS and ICP-OES have been reported for the analysis of glass and specifically as it applies to forensics. While the method of detection for these analytical approaches differs, the sample introduction and means of ionizing the analyte is the same in both instances. Although both techniques have demonstrated the ability to conduct success analysis and interpretation of this material, in general, ICP-OES has been reported in the 1980s, with few recent implementations of the technique. Initially, Hickman ${ }^{118}$ used ICP-OES to develop a method for classifying and then distinguishing glass, using elemental analysis in conjuncture with refractive index (RI). Other reports in the literature have also demonstrated the discrimination capabilities of ICP-OES for detecting variation within a glass type associated with differences in trace element concentrations $^{119}$ while also achieving a $98 \%$ discrimination power ${ }^{120}$. While ICP-OES repeatedly displayed suitable analytical merit for the elemental analysis of glass there have been more reports in the literature for ICP-MS analysis of glass, and even an ASTM method outlining sample preparation and analysis of glass using the solution ICP-MS technique ${ }^{121}$.

The instrumentation of ICP-MS more readily supported a multi-element approach to glass analysis. The spectrometers typically installed in ICP-OES instruments performed sequential analysis (one analyte at a time) and several detectors were necessary for multi-element analysis. The standardization of optical and physical properties of glass, some of which are imparted by the presence and concentration of inorganic constituents, resulted in a need to analyze an increasing number of analytes, especially trace constitutes. Simultaneous multielement analysis enhances the discrimination power that a particular technique was capable of providing. Where ICP-MS could deliver this setup, ICP-OES could not compete.

In addition ICP-MS has also easily transitioned to solid sampling by means of laser ablation. The coupling of laser ablation to ICP techniques is able to overcome many of the disadvantages of the 
above-mentioned analytical methods. Laser ablation allows for the solid sampling of the glass, minimizing sample preparation while maintaining the integrity of the sample for further analysis, if necessary. Although XRF is also used for solid sampling, although the analytical performance is element dependent (low Z elements are measured) XRF can yield limits of detection (LODs) of $\sim 50-100$ ppm for most elements ${ }^{116}$. Laser-based elemental analysis techniques are also capable of sampling very small fragments while maintaining the precision of the method.

Even with redesigned instruments capable of simultaneous detection and the ability to view the plasma from different locations, LA-ICP-OES has not been reported for the forensic analysis of glass like LA-ICP-MS. In addition to the general advantages of laser ablation, LA-ICP-OES also offers a simplified complexity of the instrumentation and management of interferences is significantly reduced in comparison to other elemental analysis techniques. LA-ICP-OES does have several advantages that would imply its applicability to forensic glass analysis it is important to stress that although there have been several reports of LA-ICP-OES in the literature for several applications, including the investigation of fundamental interactions ${ }^{68,78,122}$ as well as environmental ${ }^{123,124}$ and industrial ${ }^{125}$ applications, the complexity of data analysis persists. Although this technique has been employed in a variety of matrices, intense manipulation of the instrument report output is necessary prior to processing the transient signal data.

The studies reported here utilize LA-ICP-OES for the analysis of glass for forensic applications. The capabilities of this technique were evaluated in order to address the feasibility of applying this approach to the forensic examination of glass. A sample set of vehicle glass was analyzed in order to assess the discrimination power of LA-ICP-OES and compared to the results obtained using LA-ICP-MS, XRF and LIBS for the same sample set. In addition to analysis, the selection of match criteria for data interpretation has been emphasized in order to minimize the potential of Type I and Type II errors since the sources of the samples is known. 


\subsection{Materials and Methods}

\subsubsection{Description of Glass Standards and Samples}

\subsubsection{Use of Glass SRMs for Calibration and Quantification}

Several NIST SRMs as well as two float glass reference standards, denoted FGS-1 and FGS-2 (Bundeskriminalamt (BKA) Forensic Science Institute, Wiesbaden, Germany) were used in method development to optimize SNR, precision and concentration bias. Concentrations of the SRMs were obtained using an interactive software package, GLITTER (GEMOC, Macquarie University, Australia), which is capable of integrating the time-resolved signal and equating this to a concentration. Because FGS-1 is a matrix matched glass standard it was used as an external calibrator for signal integration for the analysis of the sample set.

To determine the applicability of this approach to calibration for LA-ICP-OES analysis of glass the bias of several glass SRMs (NIST 610, NIST 612, NIST 614, NIST 1831, FGS-2) were determined to ensure the method was capable of utilizing single point calibration. A sample calibration curve for Sr, figure 29, shows good predictability and tight precision for five replicates, demonstrated by almost unnoticeable $\mathrm{x}$ and $\mathrm{y}$ error bars which have been included on the curve. This established the applicability of single point calibration for the determination of the above listed analytes for the elemental analysis of float glass samples. 


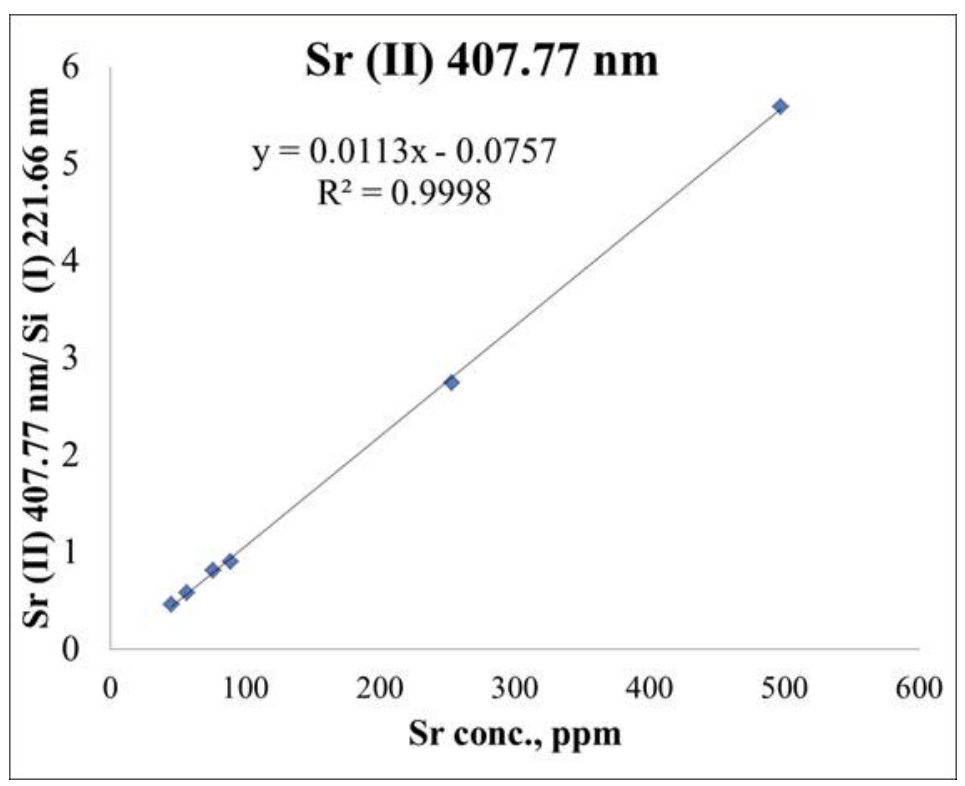

Figure 29: LA-ICP-OES glass calibration curve example.

In addition, SRM NIST 1831 was used as an independent calibration certification verification (CCV) standard. Because FGS-1 is a float glass standard the analytes of interest are present in this SRM in a similar abundance, improving the characterization of float glass samples. The concentrations can be found in table 17 for SRM FGS-1 and SRM NIST 1831 for the analytes monitored in these studies. Blank entries correspond to no certified concentration for the element listed.

Instrument performance and assessments of day-to-day variations were made using the QC charts, explained in section 4.3.5.

Table 17: Certified concentrations for glass SRMs (in ppm)

\begin{tabular}{|c|c|c|c|c|c|c|c|c|}
\hline Element & $\begin{array}{c}\text { NIST } \\
\mathbf{6 1 0}\end{array}$ & $\begin{array}{c}\text { NIST } \\
\mathbf{6 1 2}\end{array}$ & $\begin{array}{c}\text { NIST } \\
\mathbf{1 8 3 1}\end{array}$ & $\begin{array}{c}\text { NIST } \\
\mathbf{1 8 3 0}\end{array}$ & $\begin{array}{c}\text { NIST } \\
\mathbf{1 4 1 1}\end{array}$ & $\begin{array}{c}\text { NIST } \\
\mathbf{6 1 4}\end{array}$ & FGS-1 & FGS-2 \\
\hline $\mathbf{A l}$ & 10005 & 11164 & 6381 & 63.51 & 429277 & & 1500 & 7400 \\
\hline $\mathbf{B a}$ & 424.1 & 37.74 & 31.51 & & 55825 & & 40 & 199 \\
\hline $\mathbf{C a}$ & 81833 & 85262 & 58604 & 6117 & 30502 & & 60600 & 59300 \\
\hline $\mathbf{F e}$ & 457.1 & 56.33 & 610 & 109.5 & 2859 & & 580 & 2600 \\
\hline $\mathbf{L i}$ & 484.6 & 41.54 & 4.99 & & & & 6 & 29 \\
\hline $\mathbf{M g}$ & 465.3 & 77.44 & 21166 & 2351 & 5471 & & 23900 & 23400 \\
\hline $\mathbf{S r}$ & 497.4 & 76.15 & 89.11 & & 1064.3 & 45.8 & 57 & 253 \\
\hline $\mathbf{T i}$ & 434.0 & 48.11 & 113.8 & 6.6 & 333.7 & & 69 & 326 \\
\hline $\mathbf{Z r}$ & 439.4 & 35.90 & 43.35 & & & & 49 & 223 \\
\hline
\end{tabular}




\subsubsection{Description of Sample Set}

In order to address the applicability of this method to the elemental analysis of glass for forensic purposes, a sample set consisting of several vehicle glass samples was analyzed. The test set was composed of inside and outside windshields and side and rear window fragments from vehicles produced from 1995-2005. Table 18 provides a description of the samples. The particular sample set has been selected because the discrimination power achieved with other elemental analysis techniques, including LA-ICP-MS and $\mu \mathrm{XRF/EDS,} \mathrm{has} \mathrm{been} \mathrm{previously} \mathrm{reported} \mathrm{and} \mathrm{a}$ comparison of method performances will be presented ${ }^{116}$. The sample set allows for a direct comparison of several elemental analysis techniques in order to assess the appropriateness of using LA-ICP-OES for this application.

Table 18: Vehicle sample set information for LA-ICP-OES analysis

\begin{tabular}{|c|c|c|c|c|c|c|c|c|}
\hline \multirow{2}{*}{ Source } & \multicolumn{4}{|c|}{ Vehicle info } & \multicolumn{2}{c|}{$\begin{array}{c}\text { Front } \\
\text { windshield }\end{array}$} & \multicolumn{2}{c|}{$\begin{array}{c}\text { Side windows } \\
\text { window }\end{array}$} \\
\cline { 2 - 8 } & Make & Model & Year & Outer & Inner & Front & Rear & \\
\hline $\mathbf{1}$ & GMC & Jimmy & 1998 & $\# 2$ & $\# \mathbf{1}$ & & & \\
\hline $\mathbf{2}$ & Mitsubishi & Galant ES & 2000 & $\# 4$ & $\# 5$ & $\# 3$ & & \\
\hline $\mathbf{3}$ & Chevrolet & Cavalier & 2004 & $\# 6$ & $\# 7$ & & $\# 8$ & $\# 9$ \\
\hline $\mathbf{5}$ & Oldsmobile & Intrigue & 1998 & $\# 11$ & $\# 12$ & & & $\# 10$ \\
\hline $\mathbf{6}$ & Dodge & Neon & 1999 & $\# 13$ & $\# 14$ & $\# 15$ & & $\# 16$ \\
\hline $\mathbf{7}$ & Kia & Spectra GS & 2002 & $\# \mathbf{1 7}$ & $\# 18$ & & & \\
\hline $\mathbf{8}$ & Dodge & Stratus & 1998 & $\# 23$ & $\# 24$ & & & $\# 19$ \\
\hline $\mathbf{1 0}$ & Ford & $\begin{array}{c}\text { Explorer } \\
\text { Sport }\end{array}$ & 1999 & $\# 26$ & $\# 27$ & & & \\
\hline $\mathbf{1 1}$ & Ford & Expedition & 2004 & $\# 29$ & $\# \mathbf{2 8}$ & & & \\
\hline $\mathbf{1 2}$ & Ford & Eanger XLT & 2001 & $\# 33$ & $\# 35$ & $\# 34$ & & $\# 36$ \\
\hline $\mathbf{1 3}$ & Jeep & $\begin{array}{c}\text { Grd } \\
\text { Cherokee }\end{array}$ & 2001 & $\# 37$ & $\# 38$ & $\# 39$ & & \\
\hline $\mathbf{1 4}$ & Jeep & $\begin{array}{c}\text { Grd } \\
\text { Cherokee }\end{array}$ & 2001 & $\# 40$ & $\# 41$ & & & \\
\hline
\end{tabular}

The \# corresponds to the sample number; bolded numbers were missing from the sample set and not used in the analysis by LA-ICP-OES. 


\subsubsection{Preparation of Sampling Scheme of the Glass Samples}

Exposure of the surface of the glass to UV light $(254 \mathrm{~nm})$ was used to determine the non-float side since Sn fluoresces under UV light. The non-float side was then used for all analyses. Small, irregular shaped fragments were mounted using putty so that a flat, non-float side could be used for analysis. If a coating was present on the glass (side or rear window fragments) a side without coating was used for analysis. In most instances, as a consequence of sampling limitations, only single fragment analyses were performed. However, two samples were included in which multiple fragments were analyzed, and used to assess within sample variation. In addition, a single fragment from the set was analyzed to validate in order the reproducibility of the method. Regardless of the number of fragments analyzed per each sample, five replicates were performed for each fragment.

\subsubsection{LA-ICP-OES Experimental Design}

\subsubsection{LA-ICP-OES Setup and Instrumentation}

An Optima 7300 DV (PerkinElmer, Waltham, MA, USA) ICP-OES equipped with an echellebased polychromator that utilized two segmented-array charge-coupled device (SCD) detectors for ultraviolet (UV) and visible (Vis) emission coverage was used for this analysis. The general specifications of this instrument have been previously reported in chapters 3 and 4 so only information pertinent to this application will be provided. The UP-213 laser ablation unit, described in section 4.3.2.1 was used to accomplish the solid sampling of glass laser ablation parameters utilized have been outlined in section 4.3.3.1 and 4.3.3.2. Single spot analysis permits

the ability to better account for elemental layering effects in the glass by ablating into the material while minimizing possible surface contaminant analysis. In order to perform at least three analyses per fragment, it is recommended that the glass be approximately $1 \mathrm{~mm}$ in length, by at 
least $200 \mu \mathrm{m}$ wide and $100 \mu \mathrm{m}$ in thickness. A $1 \mathrm{~mm}$ size fragment allows for the three measurements to be spaced across the glass so that any surface damage (cracking) does not influence crater development during ablation. A width of $200 \mu \mathrm{m}$ is desirable to ensure that a complete crater can be formed (i.e., avoidance of cracked or blown out edges). Research by our group has also found that $50 \mu \mathrm{m}$ between the crater and the edge of the glass is sufficient to yield a completer crater. The mass removed during the ablation process is approximately $400-600 \mathrm{ng}$ from the $100 \mu \mathrm{m}$ diameter crater that is $90 \mu \mathrm{m}$ deep. It should be noted that LA-ICP-OES requires more mass to be ablated and introduced into the ICP than LA-ICP-MS, which utilizes spot size ablations $40-60 \mu \mathrm{m}$ in diameter ${ }^{109}$.

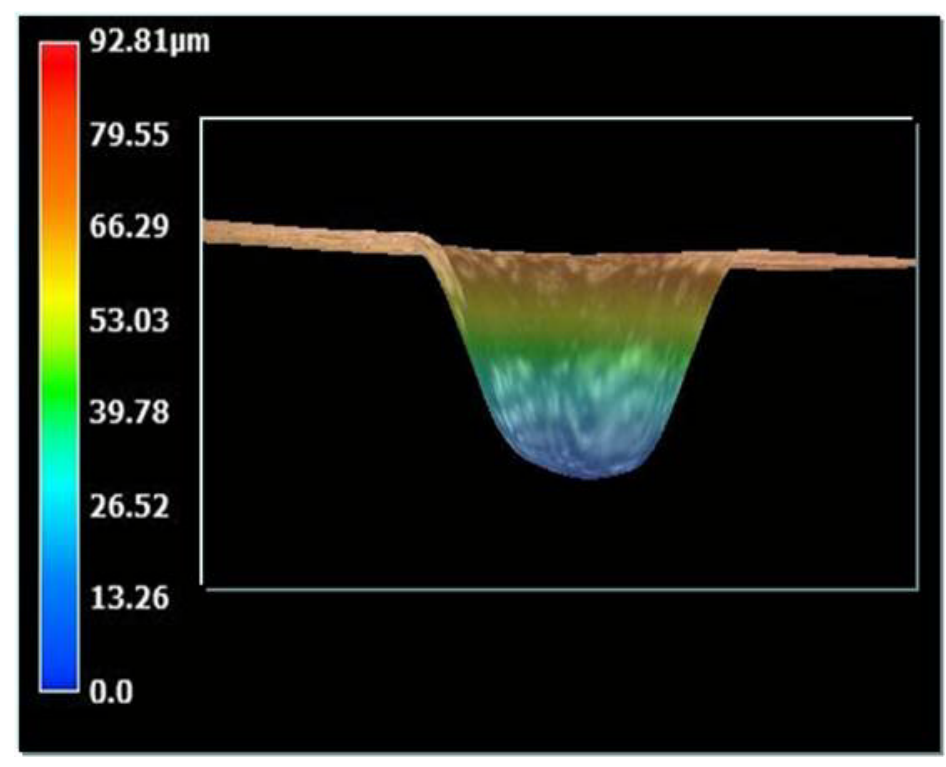

Figure 30: 3D Glass crater obtained with LA-ICP-OES method, $100 \mu \mathrm{m}$ in diameter by $92.8 \mu \mathrm{m}$ depth. This translates to $\sim 600 \mathrm{ng}$ removed.

\subsubsection{Element Menu}

The elements selected were adapted from a list of previously reported element menus that have been used for the discrimination of glass ${ }^{94,107-109,116,120,126,127}$. The element menu presented here within this work was chosen on the basis of sensitivity, presence of minimal interferences and the 
ability to achieve acceptable linear dynamic range for the application being evaluated. The LODs were compared to what has been detected in glass samples that have been analyzed and are stored in a database of elemental glass information maintained by our laboratory. The database consists of float glass sample from production plants and vehicles, container glass and headlamp glass. The vehicle glass has been collected from various junkyards in Miami Dade County, Miami, Florida. Our database includes $\sim 400$ glass samples. The database was also used to determine the dynamic range required to be able to characterize a variety of glass samples.

In addition interferences were assessed on the basis of overlapping or the presence of broad emission lines and minimal plasma background continuum radiation in the wavelength range. The selection of the emission line was influenced by 1) availability of one line over the other in the preprogrammed software and 2) the ability to fulfill the identified criteria.

On the basis of the above-mentioned requirements the following element menu presented in table 17 was chosen. Silicon has been reported as a suitable IS for elemental analysis of glass because of the abundance in which it is present in glass ${ }^{104}$. An atomic silicon emission line, Si (I) 221.66 $\mathrm{nm}$, was used as the IS, normalizing the response of the analyte to the response of the silicon to improve performance and account for pulse-to-pulse laser variations. Table 19 is broken down into detected, monitored and element menu emission lines. All emission lines detected not used for any purpose in the analysis comprised either the "detected" or "monitored" category. Emission lines that were grouped as detected were not included in the element menu because they resulted in detector saturation or the reproducibility of their detection was insufficient to warrant further exploration. Emission lines were "monitored" for several reasons including the monitoring of plasma conditions, or because these analytes (particularly $\mathrm{K}$ and $\mathrm{Mn}$ ) have been reported in the forensic analysis of glass and therefore may be useful for case specific analysis. These analytes are not included in the element menu because of the lack in sensitivity (Mn), or because the 
emission line is prone to line broadening mechanisms in the plasma at high concentrations (K). While useful, the application of these analytes is limited.

Table 19: Element emission lines used in LA-ICP-OES glass analysis

\begin{tabular}{|c|c|}
\hline Category & $\begin{array}{c}\text { Analyte Emission } \\
\text { Line }\end{array}$ \\
\hline Detected & $\mathrm{Na}$ (I) $330.23 \mathrm{~nm}$ \\
& $\mathrm{Si}$ (I) $212.42 \mathrm{~nm}$ \\
& $\mathrm{Si}$ (I) $251.61 \mathrm{~nm}$ \\
& $\mathrm{Si}$ (I) $252.85 \mathrm{~nm}$ \\
$\mathrm{Si}$ (I) $288.15 \mathrm{~nm}$ \\
\hline Monitored & $\mathrm{Ba}$ (II) $493.40 \mathrm{~nm}$ \\
& $\mathrm{Ca}$ (II) $317.93 \mathrm{~nm}$ \\
& $\mathrm{~K}$ (I) $766.49 \mathrm{~nm}$ \\
& $\mathrm{Mg}$ (II) $279.77 \mathrm{~nm}$ \\
& $\mathrm{Mg}$ (II) $280.27 \mathrm{~nm}$ \\
\hline Element Menu & $\mathrm{Al}$ (I) $396.15 \mathrm{~nm}$ \\
& $\mathrm{Ba}$ (II) $455.40 \mathrm{~nm}$ \\
& $\mathrm{Ca}$ (II) $315.88 \mathrm{~nm}$ \\
& $\mathrm{Fe}$ (II) $238.20 \mathrm{~nm}$ \\
& $\mathrm{Li}$ (I) $670.78 \mathrm{~nm}$ \\
& $\mathrm{Mg}$ (I) $285.21 \mathrm{~nm}$ \\
& $\mathrm{Sr}$ (II) $407.77 \mathrm{~nm}$ \\
& $\mathrm{Ti}$ (II) $368.51 \mathrm{~nm}$ \\
$\mathrm{Zr}$ (II) $343.82 \mathrm{~nm}$ \\
\hline
\end{tabular}

\subsection{Results and Data Analysis}

\subsubsection{Transient Signal Integration and Corresponding Concentration Evaluation}

The transient signal generated from the LA-ICP-OES data output was subjected to further data reduction using the GLITTER software package (GEMOC, Macquarie University, Australia). The program GLITTER is an interactive software package used to integrate time-resolved signal and equate the signal to a concentration. This is accomplished using an external calibrator (FGS1) of known concentration. The software uses algorithm to extrapolate the sample concentration based on the SRM response analyzed before and after the sample. The SRM must bracket the samples and be re-analyzed at least every two hours to account for the presence of any 
instrumental drift. GLITTER is capable of integrating signal for the gas blank as well as the signal attributed to laser ablation.

From the 100 seconds of data collected readings 1-15 (corresponding to the time in which they were collected) were used as the background emission signal while the data collected from $22-$ 55 were integrated for intensity of the analyte.

\subsubsection{Calculating LODs and LOQs}

The LOD of each element was calculated using the following formula:

$$
\mathrm{LOD}=3 \mathrm{~s}_{\mathrm{bckgd}}+\mathrm{x}_{\mathrm{bckgd}}
$$

where "s" corresponds to the standard deviation of the background (also referred to as noise) and " $\mathrm{x}$ " corresponds to the average background signal, both of which were obtained within the region of the emission line of interest. This formula determines the LOD in counts or intensity, which was then related to a concentration using a ratio comparing the LOD information to that of a sample.

$$
\frac{\text { Intensity }_{\text {sample }}}{\text { Concentration }_{\text {sample }}}=\frac{\text { Intensity }_{L O D}}{\text { Concentration }_{L O D}}
$$

Three of the four variables of this relationship are known, allowing the concentration at the LOD to be calculated. The process was repeated for all samples and compiled so that a representative LOD assessment could be made. To determine the validity of this approach samples from the glass database were analyzed and compared to the LOD value obtained here.

The LOQ was calculated using ten times the standard deviation (s) of the background signal in place of three used in equation 16. 


\subsubsection{Performance of SRM NIST 1831}

The precision and bias of the method was evaluated on the basis of SRM NIST 1831

performance. Each analysis was measured using the average and standard deviation of five replicates made both at the beginning and end of each study. A comparison to the reported values provided by NIST, the ASTM method for glass analysis (ASTM method E 2330-04) ${ }^{121}$ or other studies that evaluated the NIST glass series ${ }^{128}$ was made. The analysis was deemed useable if the bias did not vary more than $+/-10 \%$ from the reported value, and had a precision $<10 \%$;

although most elements performed with a precision below $8 \%$. Table 20 provides a comparison of the method performance based on the precision and bias of NIST 1831 over 60 replicates, spanning a month of analyses. The LA-ICP-OES method is also compared to the performance of the LA-ICP-MS for the overlapping element menus. The LA-ICP-MS results reported in table 20 were obtained from average analyses of NIST 1831 made of several years in our laboratory.

Table 20: Analytical performance of SRM NIST 1831

\begin{tabular}{|c|c|c|c|c|c|c|c|}
\hline \multicolumn{2}{|c|}{ Analyte information } & \multicolumn{3}{|c|}{ LA-ICP-OES } & \multicolumn{3}{|c|}{ LA-ICP-MS } \\
\hline Element & $\begin{array}{c}\text { Reported } \\
\text { value } \\
\left(\mu \mathrm{g} \mathrm{g}^{-1}\right)\end{array}$ & $\underset{\left(\mu \mathrm{g} \mathrm{g}^{-1}\right)}{\text { Average }}$ & $\begin{array}{l}\text { Bias } \\
(\%)\end{array}$ & $\begin{array}{c}\text { Precision } \\
(\%)\end{array}$ & $\begin{array}{c}\text { Average } \\
\left(\mu \mathrm{g} \mathrm{g}^{-1}\right)\end{array}$ & $\begin{array}{l}\text { Bias } \\
(\%)\end{array}$ & $\begin{array}{c}\text { Precision } \\
(\%)\end{array}$ \\
\hline $\mathrm{Al}$ & $6381^{b}$ & 6232 & -2.3 & 1.4 & 6828 & 7.0 & 12.0 \\
\hline $\mathrm{Ba}$ & $31.5^{\mathrm{c}}$ & 29.1 & -7.6 & 3.0 & 29.1 & -7.6 & 3.5 \\
\hline $\mathrm{Ca}$ & $58604^{b}$ & 58020 & -1.0 & 1.8 & 58391 & -0.4 & 2.6 \\
\hline $\mathrm{Fe}$ & $610^{\mathrm{b}}$ & 581 & -4.8 & 2.8 & 530 & -13.1 & 12.4 \\
\hline $\mathrm{Li}$ & $4.99^{\mathrm{a}}$ & 5.21 & 4.5 & 6.8 & 5.13 & 2.8 & 2.9 \\
\hline $\mathrm{Mg}$ & $21166^{b}$ & 21177 & 0.1 & 1.1 & 25809 & 21.9 & 6.5 \\
\hline $\mathrm{Sr}$ & $89.1^{\mathrm{c}}$ & 85.9 & -3.6 & 6.6 & 75.9 & -14.8 & 2.4 \\
\hline $\mathrm{Ti}$ & $114^{\mathrm{b}}$ & 108 & -5.3 & 6.6 & 135.5 & 18.9 & 15.0 \\
\hline $\mathrm{Zr}$ & $43.4^{\mathrm{c}}$ & 37.5 & -13.6 & 9.5 & 31.3 & -28.3 & 2.3 \\
\hline
\end{tabular}

${ }^{a}$ Historical data from a single lab over a one year period

${ }^{\text {b }}$ Certified by NIST

${ }^{\mathrm{c}}$ Reported in ASTM method E 2330-04, not certified 
The differences in bias values obtained in each method can most likely be attributed to the different single point calibration glass standards used; NIST 612 and FGS-1 were the single point calibration standards utilized by the LA-ICP-MS and LA-ICP-OES methods, respectively. The elemental concentrations of these glass standards vary, which would be reflected in the accuracy of the extrapolation capabilities for large differences in calibrator and verification standard concentrations. However, FGS-1 is a more appropriate single point external calibrator for the analysis of float glass samples because it is matrix matched. A factor that may play a role in why it is not so readily used by analysts as a calibrator SRM because it has previously been difficult to obtain because it is not a glass SRM that is produced in large batches like NIST glasses.

However, more important than bias, is the ability for the method to see real differences in samples that contribute to the informing or discrimination power of the method.

The similar demonstration in precision of each method in respect to a broad range of concentrations implies that LA-ICP-OES is capable of producing comparable analytical performance for the elemental analysis of glass. The tight range of precision and bias values also suggests minimal intra and inter-day variation.

\subsubsection{Analytical Performance of the LA-ICP-OES Method}

In addition to measuring the method performance utilizing the response of SRM NIST 1831, the applicability of this method for the elemental analysis of glass was assessed using the limits of detection and quantitation (LOD and LOQ, respectively) presented in table 21 . The performance of most elements were capable of yielding LODs and LOQs well below typically encountered sample concentrations, as determined by the analysis of over 400 vehicle and architectural windows from a glass database maintained by our laboratory. Although the LOQs of both lithium (Li) and zirconium (Zr) fall in the working sample concentrations, the particular values did fall 
close to the minimum, decreasing the probability that the sensitivities of these analytes would be problematic in later analyses.

Table 21: LODs and LOQs for the LA-ICP-OES analysis of glass

\begin{tabular}{|c|c|c|c|}
\hline Emission line (nm) & $\begin{array}{c}\text { Limit of detection } \\
\left(\boldsymbol{\mu g ~ g}^{-\mathbf{1}}\right)\end{array}$ & $\begin{array}{c}\text { Limit of quantitation } \\
\left(\boldsymbol{\mu g ~ g}^{-\mathbf{1}}\right)\end{array}$ & $\begin{array}{c}\text { Typical sample } \\
\text { Range }\left(\boldsymbol{\mu \mathbf { g ~ g ~ } ^ { - 1 }}\right)\end{array}$ \\
\hline Al I 396.15 & 3.6 & 12.1 & $298-11,940^{\mathrm{a}}$ \\
\hline Ba II 455.40 & 0.6 & 1.7 & $3-384^{\mathrm{b}}$ \\
\hline Ca II 315.88 & 559 & 1862 & $46,086-69,767^{\mathrm{b}}$ \\
\hline Fe II 238.20 & 13.1 & 39.3 & $461-6063^{\mathrm{a}}$ \\
\hline Li I 670.78 & 0.38 & 1.31 & $0.8-7.0^{\mathrm{a}}$ \\
\hline Mg I 285.21 & 8.1 & 26.9 & $6273-51,076^{\mathrm{b}}$ \\
\hline Sr II 407.77 & 0.2 & 0.7 & $19-576^{\mathrm{b}}$ \\
\hline Ti II 368.51 & 6.9 & 23.1 & $39-3226^{\mathrm{b}}$ \\
\hline Zr II 343.82 & 7.1 & 22.3 & $19-269^{\mathrm{b}}$ \\
\hline
\end{tabular}

${ }^{a}$ Determined from actual measurements made of 127 soda-lime glass samples consisting of vehicle and architectural windows.

${ }^{b}$ Determined from actual measurements made of 286 soda-lime glass samples from vehicle and architectural windows.

Table 21 reiterates that LA-ICP-OES is capable of achieving the analytical performance necessary for the analysis of trace and minor elements in glass samples for the element menu presented.

\subsubsection{Statistical Analysis of Vehicle Glass Sample Set}

\subsubsection{Considerations of Data Interpretation}

\subsection{Discrimination Techniques}

In order to make a direct comparison to the previously published data, the resulting LA-ICP-OES concentration values of the sample glass set were statistically compared using successive hypothesis tests derived from pair-wise comparisons. Pair-wise comparisons analyze the results of one sample against another for a single analyte. In all statistical hypothesis comparisons a null hypothesis must be defined. For the analysis of this data the null hypothesis has been defined as: 


$$
H_{0}: \bar{x}_{1}=\bar{x}_{2}
$$

where and corresponds to the means of the two sample populations being compared. When the null hypothesis is not rejected, that supports the hypothesis that there is no significant difference between the two means at the $95 \%$ confidence level. The pair-wise comparisons applied analysis of variance (ANOVA), followed by Tukey's post hoc test using SYSTAT 11 statistical software package (Systat Software, Inc., Chicago, IL, USA). Pairwise comparisons using ANOVA are used to test whether two or more means are equal by comparing the variances ${ }^{129}$. The total variation with the measurement can be separated into sources which can be controlled or explained and the experiment and sources of which the analyst has no control ${ }^{129}$. It is the variance that is not the result of random error that is used to determine whether or not the means are equal. The Tukey's post hoc test is applied following the ANOVA and are used to determine which means differ. Pairs found to be indistinguishable by this method were further examined using a student's t-test at the $95 \%$ confidence interval. The information was then used to determine the discrimination power of the method on the basis the total number of possible pairs and the number of indistinguishable pairs. The total number of pairs can be determined using $n(n-1) / 2$ where $n=$ to the number of samples ( 820 pairs in this study).

Because the selection of match criteria can exploit the ability of the method to measure analytes with very good precision or may be overly sensitive to sample microheterogeneity other approaches were utilized. The use of ANOVA and even t-test can falsely misinterpret the above instances as real differences. Therefore, besides presenting the data using ANOVA with Tukey's post hoc test, followed by the t-test at a $95 \%$ confidence interval in order to compare with previously published work ${ }^{116}$, a more broad match criteria have also been investigated. The use of a more narrow match criteria, although prone to false interpretation of microheterogeneity was used because the previously reported elemental analysis techniques utilized this approach ${ }^{116}$. 
While broader match criteria may be more suitable for forensic comparisons of glass, the application of ANOVA followed by a t-test, permits the direct comparison to the other techniques.

The data (both LA-ICP-MS and LA-ICP-OES) have also been analyzed using the average analyte concentration \pm two, three and four standard deviations $(2 \sigma, 3 \sigma, 4 \sigma)$ as additional match criteria. Each sample was compared to all other samples in order to determine the number of indistinguishable pairs. A comparison consisted of comparing the average, $2 \sigma, 3 \sigma$, or $4 \sigma$ analyte for one sample "sample 1" to the average of the analyte of another sample to determine of the second sample "sample 2 " falls within the established match range $\left(\bar{x}_{\text {sample }} \pm n \sigma\right.$, where $n=2,3$ or 4 . Since either sample for the particular test set being compared could be considered the known forensic sample or the unknown fragment retrieved as evidence the sample comparison was conducted in both manners. The samples initially considered "sample 1" and "sample 2" were reversed and the average and match ranges were evaluated. An indistinguishable pair was determined when both comparisons (initial and reverse) resulted in an indistinguishable result. Only one analyte had to be distinguishable for the fragments to be considered different using this match criterion, whereas all analytes had to be indistinguishable for the glass fragments to be the same.

In addition to the above fixed match criteria, a modified $4 \sigma$ using a fixed relative standard deviation $(3 \%)^{126}$ was also included in the statistical analysis. The approach uses an established level of precision (3\%) and adjusts analytes that yield smaller RSDs to a minimum of 3\% RSD (or the actual RSD value, if greater). Again, each sample was evaluated against every other sample in the test set in order to determine the number of indistinguishable pairs. The same approach to making comparisons between two samples are described above was used for this criterion as well. 


\subsection{Types of Error and Rules for Data Interpretation}

For the discrimination of known and unknown samples, there are two types of errors. Type I errors are the result of falsely excluding data that should not be excluded; type II errors are the result of falsely including data that should not be included. The worst type of error concerning forensic analysts is type II error so precautions should be taken to minimize the type of error. When a $95 \%$ confidence interval (CI) is employed in statistical testing this implies that there is a $5 \%$ probability of a type I error. As the confidence interval is varied $90 \% \mathrm{CI}$ to $99 \% \mathrm{CI}$ this has an effect on the probability of committing a type I error, resulting in $10 \%$ and $1 \%$ chance of occurrence, respectively. However, it should be noted that decreasing or increasing the possibility of type I errors has the opposite effect on the type II error rate.

Because this was a well-documented sample set (model and production year of car and information regarding specific glass source, i.e., inside or outside windshield pane were all available) insight regarding the possibility of Type I errors (false exclusions) and Type II errors (false inclusions) can also be made. Prior to the start of data analysis certain rules regarding the interpretation of statistical evaluations should be made and kept for the duration of the analysis and interpretation.

Here, we are defining false inclusions to consist of false associations of glass from two different sources. Two different sources are then defined as two completely different sources i.e., two windshields from two different vehicles. On the other hand, an example of the same source would comprise both inside and outside windshield glass originating from the same vehicle. Because the probability that the inside and outside windshields share a similar production date in the same manufacturing plant, and in turn have indistinguishable elemental profiles is more likely than not, it is logical that associations of this nature can be made. The ability to associate glass samples of this type will be used to assess the potential of type I errors. Several duplicate samples were 
analyzed (same fragment repeatedly, same source different fragment) in order to determine measurement reproducibility of small sample fragments. The duplicate samples were analyzed throughout the run as a secondary control to assess the ability of the data analysis software to account for instrumental drift during the analysis of small fragments. The various types of duplicate samples described are also being used to determine the possibility of type I errors. Type II errors are being evaluated by determining the number of falsely associated glass samples from different sources, as defined above.

\subsubsection{Interpretation of the Sample Set}

The data were analyzed using two methods: 1) applying only the five most discriminating elements ( $\mathrm{Sr}, \mathrm{Al}, \mathrm{Mg}, \mathrm{Ba}, \mathrm{Zr}$ ) so that the number of elemental factors would be the same for LAICP-MS ${ }^{116}$ and LA-ICP-OES and 2) using the entire element menu. The statistical analysis of the LA-ICP-OES data yielded four indistinguishable pairs regardless of the number of elements compared. Table 22 presents a description of the indistinguishable pairs encountered using ANOVA with Tukey's post hoc test and the t-test at the $95 \%$ confidence interval (C.I.) as well as the resulting indistinguishable pairs from other techniques.

All indistinguishable pairs originated from the same source, i.e., from the inside and outside windshield panes from the same vehicle. The fact that the same vehicle was the source of each indistinguishable pair identified suggests that the manufacture date and location of the glass is likely the same. Although this is a plausible explanation for the outcome of this particular experiment, this scenario was not observed in all like instances, i.e., all same source pairs in table 22 were not indistinguishable by both ICP techniques. There were a total of 11 possible pairs of inner and outer windshield pairs that should have been indistinguishable, although fewer (4 pairs) were encountered suggesting possible Type I errors. In order to determine if these cases are the product of a Type I error it is important to evaluate whether the differences observed in the 
expected indistinguishable pairs are the result of real differences or, reflect the influence of match criteria selection.

Table 22: Resulting indistinguishable pairs from the vehicle glass set using ANOVA with Tukey's and the t-test at the $95 \%$ C.I.

\begin{tabular}{|c|l|l|l|l|}
\hline Pair \# & Vehicle make & Vehicle model & Year & Sample location \\
\hline \multirow{2}{*}{$1^{\mathrm{a}}$} & Chevrolet & Cavalier & 2004 & side window \\
\cline { 2 - 5 } & Chevrolet & Cavalier & 2004 & rear window \\
\hline \multirow{2}{*}{$2^{\mathrm{a}, \mathrm{b}, \mathrm{c}}$} & Chevrolet & Cavalier & 2004 & outside windshield \\
\cline { 2 - 5 } & Chevrolet & Cavalier & 2004 & inside windshield \\
\hline \multirow{2}{*}{$3^{\mathrm{a}, \mathrm{b}}$} & Oldsmobile & Intrigue & 1998 & outside windshield \\
\cline { 2 - 5 } & Oldsmobile & Intrigue & 1998 & inside windshield \\
\hline \multirow{2}{*}{$4^{\mathrm{a}, \mathrm{b}}$} & Dodge & Neon & 2000 & outside windshield \\
\cline { 2 - 5 } & Dodge & Neon & 2000 & inside windshield \\
\hline \multirow{2}{*}{$5^{\mathrm{a}, \mathrm{b}}$} & Chevrolet & Cavalier & 2003 & outside windshield \\
\cline { 2 - 5 } & Chevrolet & Cavalier & 2003 & inside windshield \\
\hline \multirow{2}{*}{$6^{\mathrm{a}, \mathrm{b}, \mathrm{c}}$} & Dodge & Stratus & 1998 & outside windshield \\
\cline { 2 - 5 } & Dodge & Stratus & 1998 & inside windshield \\
\hline \multirow{2}{*}{$7^{\mathrm{a}, \mathrm{c}}$} & Ford & Expedition & 2004 & inside windshield \\
\cline { 2 - 5 } & Ford & Expedition & 2004 & outside windshield \\
\hline \multirow{2}{*}{$8^{\mathrm{a}, \mathrm{c}}$} & Jeep & Grd. Cherokee & 2001 & outside windshield \\
\cline { 2 - 5 } & Jeep & Grd. Cherokee & 2001 & inside windshield \\
\hline
\end{tabular}

${ }^{\text {a }}$ Corresponds to an indistinguishable pair found by $\mu \mathrm{XRF}$

${ }^{\mathrm{b}}$ Corresponds to an indistinguishable pair found by LA-ICP-MS

${ }^{c}$ Corresponds to an indistinguishable pair found by LA-ICP-OES

The broader match criteria was also applied. Table 23 depicting the results of the $\sigma, 3 \sigma$ and $4 \sigma$ shows the improvement or decrease in the number of type I errors. The use of "IN" in the table corresponds to "indistinguishable."

Two associations between the side and rear windows from the same vehicle were found by comparing the data collected from both techniques. In addition, both techniques also failed to associate the same three pairs of inner and outer windshields from the same vehicle, and LA-ICPMS also failed to associate an additional pair. A broader match criterion improves the ability to make same source associations, improving the informing power of the technique. The application 
of the $4 \sigma$ match criterion increased the number of inner and outer windshield associations from the same vehicle over that which was achieved using ANOVA with Tukey's post hoc test (4 to 8 pairs). The improvement in the ability to make associations implies that a broad match criterion is able to detect real differences between samples rather than micro-heterogeneity within a source, which is corroborated by the fact that all indistinguishable pairs encountered were associations from same vehicle. Therefore, both techniques have the ability to detect differences from two different sources (two different vehicles).

Table 23: Indistinguishable pairs obtained when broader match criteria is used to interpret

\section{LA-ICP-OES glass data}

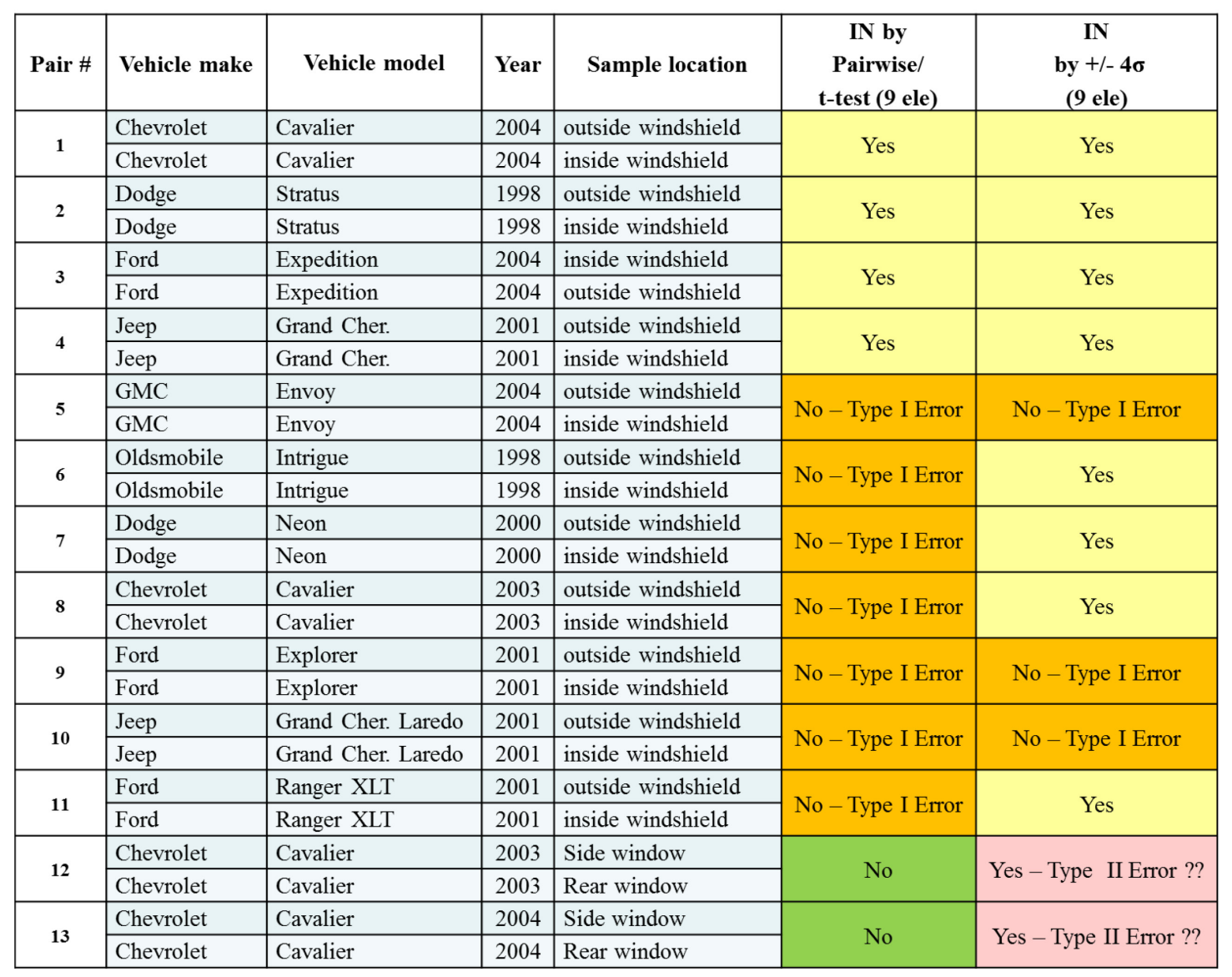


The similarity of information obtained from LA-ICP-MS and LA-ICP-OES suggests that the results are not technique dependent. LA-ICP-OES is capable of providing comparable elemental information for glass analysis as the more established and reported LA-ICP-MS results.

In addition the modified approach (fixed RSD) was compared to the $2 \sigma, 3 \sigma$ and $4 \sigma$ approach, yet it did not improve the number of same-source false exclusions. It did, however, introduce an additional associated pair that did not originate from the same vehicle. The additional pair had been distinguishable when the other statistical analyses discussed had been used. The pair associated here was the only instance of two different vehicles being associated. The concept of a threshold precision level was first demonstrated using LA-ICP-MS data and a significant amount of historical data. While a fixed precision approach may still be applicable to LA-ICP-OES, more data may be required when establishing threshold levels.

When interpreting the results obtained in this study the sampling of the test set must be taken into consideration. The test set being used to evaluate the instrumentation is slightly limited in that very few fragments from each source were available for analysis. The analysis of additional fragments, as recommended previously in the literature ${ }^{94}$ is another technique that would account for microheterogeneity of the source that broader match criteria cannot account for using single fragment comparisons.

On the basis of the data analysis from the experiments presented here, the selection of appropriate match criteria is critical for accurate assessment of associations that are the result of differences that are present in the glass, not from microheterogeneity that the selected data handling technique did not account for. 


\subsection{Discussion and Conclusions}

The current studies demonstrate that LA-ICP-OES can be used as an alternative technique to other elemental analysis techniques for the forensic analysis of glass. The coupling of laser ablation to ICP-OES has resulted in analytical figures of merit and discrimination capabilities comparable to LA-ICP-MS, the elemental analysis "gold standard".

On the basis of the performance of the test sample set presented here, a broad match criterion of either $4 \sigma$ or a modified $4 \sigma$ is recommended to account for sample heterogeneity in the elemental comparison of glass fragments by LA-ICP-OES and the "gold standard.". A broad match criterion is capable of achieving an excellent discrimination power and will minimize the potential for type I errors. It has also been demonstrated that the type II error rate, while dependent on the potential of type I errors, was not sacrificed with the selection of a broader match criterion. In addition, the results stress the importance of considering sampling strategy to account for the inherent heterogeneity of the source of glass being interrogated.

Although LA-ICP-MS and LA-ICP-OES differed in element menus, both techniques were capable of achieving similar discrimination abilities, with LA-ICP-OES associating an additional same source inner/outer windshield glass sample pair when a match criteria of $( \pm 4 \sigma)$ was applied. The $( \pm 4 \sigma)$ match criterion improved the ability to associate same source pairs (inner and outer windshields) for both techniques. It also did not result in any two different vehicle associations. The match criterion was able to better account for actual differences between glass sources. It was shown that when the glass originated from the same vehicle, associations were made that supports the hypothesis of similar production dates within the same manufacturing plant.

The similarity in information obtained from LA-ICP-OES, both in analytical and sample discrimination performance, coupled with the reduced instrument complexity, cost and 
maintenance suggests that LA-ICP-OES can compete with other instrumentation currently employed for the forensic analysis of glass including the use of LA-ICP-MS, the current elemental analysis "gold standard.".

\subsection{APPLICATION OF LA-ICP-OES AND LIBS FOR THE ELEMENTAL ANALYSIS OF UNPROCESSED COTTON}

\subsection{Overview}

Cotton is a natural fiber grown throughout the world. It is a common commodity exported and imported from several countries, the U.S. included. Different types of cotton are grown in different geographic regions. These different cotton types have of differing quality and end uses. Currently, only paper documentation is used to identify the geographic origin and thus cotton quality. The current chapter evaluates an independent method of geographically provenancing the cotton.

There are several elemental analysis techniques that can be utilized for geographic provenancing of unprocessed cotton by means of the metal content present in the fibers. Atomic emission techniques offer advantages over other analytical instrumentation in that they are often less complex in configuration and detection, resulting in less expensive bench-top analysis. These attributes makes the exploration of atomic emission attractive for conducting elemental analysis, placing it within reach of laboratories that cannot afford LA-ICP-MS. LIBS and LA-ICP-OES are atomic emission techniques that differ in the manner in which the emission is produced and detected. The exploration of the relationship between elemental composition and geographic origin is presented while addressing difference in performance for two analytical techniques. Multivariate statistics introduces exploratory data analysis, as a means of testing the hypotheses 
proposed by this research. In addition the sampling strategy of the cotton matrix is presented and the development of a matrix matched unprocessed cotton standard is explained.

\subsection{Introduction}

\subsubsection{Cotton}

Cotton is a natural fiber used in numerous textile products ranging from apparel to industrial goods. In 2008 cotton accounted for over $43 \%$ of the total fiber consumption worldwide ${ }^{2}$. Although the plant is a wild shrub, it thrives as a harvested crop in tropical climates in areas including North and South America and Asia, among other locations ${ }^{130}$. There are many species of cotton, which all belong to the genus Gossypium. Of these, only a few species are grown as field crops and possess the qualities of fiber strength, length, and uniformity needed for use in the textile industry. Species found in commercial textiles include G. hirsutum, G. barbadense, $G$. arboreum, and $G$. herbaceum ${ }^{131}$. Within the species there are several varieties. A variation arises when cotton species have been bred together to form a hybrid ${ }^{131,132}$. Varieties of cotton can also be referred to as cotton varieties.

The structure of the cotton plant is the same, regardless of the species or variation. The cotton itself is actually considered a fibrous seed hair. It is grown in a part of the plant known as the boll ${ }^{131}$. The boll provides a safe environment for cotton, keeping it dry from rain and clean from wind particulates. The fibers initially grow in length. Once lengthwise growth of the fibers stops, the fibers will increase in strength, forming cellulose layers, similar in appearance to tree rings ${ }^{131}$. Figure 31 depicts the cotton boll and fiber composition. The cotton boll slowly opens as the fibers mature (top left, fig. 31) ${ }^{133}$; completely exposed, the boll is ready for harvest (top right, figure 31$)^{134}$. The composition of the fibers consists of several layers of complex organic 
constituents that increase as the fiber matures throughout the growing season (bottom, figure $31)^{131}$.

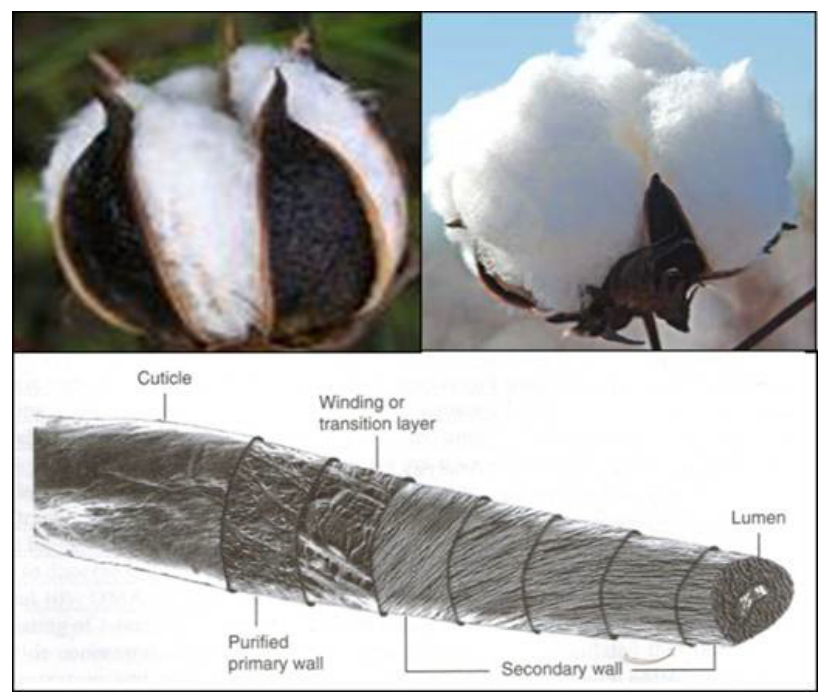

Figure 31: Cotton boll and fiber composition.

Just like any other plant, cotton requires nutrients and water for optimal growth. As the plant matures through the growing season the water source shifts from nearly surface to utilizing ground water to obtain nutrients ${ }^{135,136}$. Ground water refers to water that is not directly in contact with a body of water - lake, river, stream etc. The water is typically "run-off” from other water sources and precipitation that has penetrated the ground. The ground water also serves as a mode of transportation for nutrients to reach the plant. In the climate regions where cotton is grown, ground water is similar to that of the precipitation and nearby areas associated with water recharge ${ }^{137}$. Both the water and nutrients are taken up by the roots of plant. The roots of cotton are fairly deep (eventually $>90 \mathrm{~cm}$ from the surface) and expansive ${ }^{135}$. The root system allows for water and nutrient uptake to occur at increasing depths as the plant matures. Following uptake the nutrients are translocated throughout the plant via xylem. Concentrations of the nutrients in the plant are not evenly distributed. The transportation of nutrients corresponds to the mobility of the nutrient-containing compound and the intended use of the nutrient in the plant ${ }^{136}$. Transportation 
of nutrients follows the principles of translocation. Translocation is the movement of nutrients and photosynthesis products using channels located throughout the plant tissue ${ }^{136}$. Thus the ability to detect all nutrients present in a specific plant in every portion of the plant anatomy is not feasible. Element or metal concentration in the actual cotton fiber is much lower than the rest of the plant ${ }^{138,139}$. These trace elements can be present in a wide range $(1-6500 \mathrm{ppm})^{131,140}$.

Elements taken up by the plant fit one of two categories: mineral nutrient or nonessential nutrient ${ }^{141}$. Mineral nutrients are substances that are required to establish and maintain a healthy life for organisms and include both macro and micronutrients. The nutrients that are needed in large amounts are known as macronutrients, whereas those required in much lower concentrations are referred to as micronutrients. Macronutrients essential to growth include $\mathrm{Ca}, \mathrm{Mg}, \mathrm{N}, \mathrm{P}, \mathrm{K}$ and $\mathrm{S}^{142}$. Micronutrients vary from plant species to species depending on the specific role but, generally speaking, this group includes $\mathrm{B}, \mathrm{Cu}, \mathrm{Fe}, \mathrm{I}, \mathrm{Mn}$ and $\mathrm{Zn}$ among other elements ${ }^{142}$. In addition, if some of the elements are unavailable in the mobile component on the soil, other elements can be taken up by the plant. The plant cannot control the uptake of metals in solution, i.e., selective uptake. This, then also results in the uptake of nonessential elements that are also dispersed throughout the plant material. It is hypothesized that both mineral and nonessential elements will present in the metal composition of the cotton boll.

The elemental profile of the cotton relies on a plant-soil-water relationship. The relationship is directly related to the bioavailability of the particular nutrient ${ }^{141}$. The bioavailability is correlated to the mineralogy of the soil and parent rock material as well as the presence of ground water ${ }^{136}$. Therefore, the elemental profile, it is hypothesized, is related to the geographic region in which the cotton was grown.

Some research regarding the nutrient content or elemental profile of cotton had been conducted prior to the start of this dissertation. It has been previously reported that the elemental profile of 
unprocessed cotton is composed of attributes relating to plant nutrition ${ }^{85}$ as well as some environmental contaminants ${ }^{138,143}$. Domestic cotton was analyzed by atomic absorption (AA) and ICP-OES ${ }^{85}$ and reported differences in element concentration by region. These authors reported that cotton grown in California contained higher K content than cotton grown in the eastern U.S. however, no statistical evaluation was conducted to quantify any differences. Another, more recent study ${ }^{85}$ analyzed non-domestic cotton for a comparison of the elemental profile to that of domestic cotton. Samples from Africa, China, Greece, Syria and Russia were included in this study. Generally speaking, when comparing specific elemental contributions of $\mathrm{Ca}, \mathrm{Cu}, \mathrm{Fe}, \mathrm{K}$, $\mathrm{Mg}, \mathrm{Mn}$ and $\mathrm{Zn}$ foreign grown cotton had a more variable elemental profile. However, again, no statistical assessments were performed on these data.

The previously conducted elemental analysis did not extensively evaluate the relationship between geographic region and the elemental profile in unprocessed cotton. Research conducted within the scope of the present dissertation expands the basic knowledge of the metal content in cotton to provide a method to accomplish geographic provenancing of this commodity.

\subsubsection{Principles of Geographic Provenancing}

Geographic provenancing is a term used to describe the ability to relate a material to the geographic region from which it originated ${ }^{144}$. The ability to accomplish provenancing using both elemental and stable isotope ratio analysis, separately or together has been demonstrated

throughout the literature ${ }^{145-147}$. Geographic provenancing is becoming an important method of establishing associations in the forensic community. Natural products especially possess inherent information from the region or location from which it originated that, without negatively impacting the good, cannot be removed or tampered with. In addition, because geographic provenancing has been applied to a variety of matrices and has been done so successfully strengthens the validity when these associations are made. 


\subsection{Materials and Methods}

\subsubsection{Description of Samples and Sampling Scheme}

\subsubsection{Cotton Sampling Strategy}

As previously demonstrated, cotton is an abundant crop in the U.S. and several other countries. To ease the transportation of this unprocessed commodity this good is compressed into a dense $55 "$ x 21 " x 33" bale, weighing approximately $375-515 \mathrm{lbs}^{131}$. Although a bale was not available for sampling at any point in this research the developed sampling strategy and statistical interpretation of data was designed to accommodate the heterogeneity that could be encountered within such a massive sample source. The ultimate goal of this research was not to distinguish or associate a single sample to another, but to determine the assignment of a sample to a particular category, in this instance a geographical region, which was taken into consideration when sampling strategies were developed.

The currently employed cotton sampling strategy was developed using a set of samples in which a large amount of cotton was available ( $200 \mathrm{~g} /$ sample). The sample set was obtained courtesy Cotton Incorporated ${ }^{\circledR}$. The samples in table 24 were used to mimic the heterogeneity within a cotton bale.

Unprocessed cotton contains a significant amount of debris from the harvesting of the crop, which needs to be removed prior to further steps in the sampling process. The cleanup of the cotton was accomplished using plastic tweezers to remove such debris. The cleanliness of the cotton was then confirmed via visual inspection employing a stereomicroscope (KL300 LED, Leica Microsystems Inc., Buffalo Grove, IL, USA). 
Table 24: Cotton Inc. sample set

\begin{tabular}{|c|c|}
\hline Location & Harvest \\
\hline \multirow{2}{*}{ Arizona } & 2005 \\
\cline { 2 - 2 } & 2006 \\
\hline \multirow{2}{*}{ California } & 2005 \\
\cline { 2 - 2 } & 2006 \\
\hline \multirow{2}{*}{ Mississippi } & 2005 \\
\cline { 2 - 2 } & 2006 \\
\hline \multirow{2}{*}{ North Carolina } & 2005 \\
\cline { 2 - 2 } & 2006 \\
\hline \multirow{2}{*}{ New Mexico } & 2005 \\
\cline { 2 - 2 } & 2006 \\
\hline \multirow{2}{*}{ Texas } & 2005 \\
\cline { 2 - 2 } & 2006 \\
\hline
\end{tabular}

To evaluate an appropriate mass of cotton to utilize for cleanup and subsequent sampling the Texas 2005 sample was split into 11 approximately equal portions $(\sim 15-20 \mathrm{~g})$. The entire portion was subjected to the cleanup procedure. From each subsample, $10 \mathrm{~g}$ were then carried through the pellet making process (see section 6.3.1.2). From this, one cotton pellet sample was produced and used to characterize each of the ten splits. Figure 32 portrays the comparison of results obtained from each pellet for several elements. The narrow spread of variation suggests that the approach of selecting cotton from throughout the entire bulk material and maintaining this representative sampling on smaller scales is sufficient to accomadating heterogeneity issues that can be encountered in environmental samples.

On the basis of these results it was determined that attention to achieving a representative sampling strategy was critical to accurately characterizing the matrix. It is established in figure 32 that if a representative sampling strategy is maintained, the bulk cotton sample is characterized by sampling a subsample that is $\sim 1 / 10$ the mass of the bulk. As a result of the variations in sample size $(10 \mathrm{~g}-500 \mathrm{~g}$, typical $=\sim 100 \mathrm{~g})$ for the cotton sample population used in this research a standardized sampling design was developed. This design consisted of utilizing a standard $20 \mathrm{~g}$ for cleanup, regardless of total amount, that was collected from throughout the bulk material. For 
samples consisting of $>20 \mathrm{~g}$ of bulk material, the entire sample was cleaned and used in the additional sampling stages in which the end product are cotton pellet(s) .

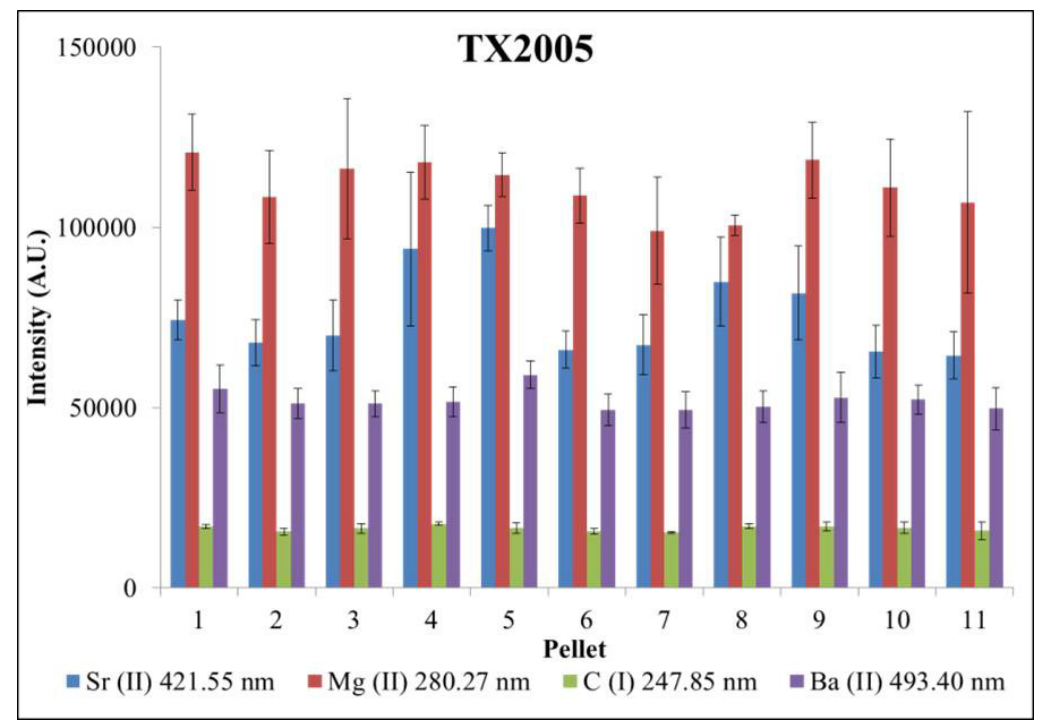

Figure 32: Heterogeneity observed in large batches of cotton by LIBS.

\subsubsection{Cotton Pellet Preparation}

The application of laser-based sampling for the elemental analysis of unprocessed cotton requires that the matrix be both homogenous and compacted so that it can withstand repeated laser-sample interactions. Milling the cotton fibers to a powder and then pressing this powder into a pellet satisfy these two requirements.

The generation of a powder is a method of homogenizing the matrix on a micro level. Pressing the powder into a pellet yields a dense sample of fine $(>20 \mu \mathrm{m})$ grains, which is ideal for laser sampling methods.

The cotton analysis required a high speed ball mill (MM200, Retsch, Clifton, NJ, USA) with 10 $\mathrm{mL}$ tungsten carbide vials each equipped with a single $10 \mathrm{~mm}$ ball was used to homogenize the cotton. From the above-described $20 \mathrm{~g}$ of clean cotton, $5 \mathrm{~g}$ were milled to a fibrous powder $(<50$ 
$\mu \mathrm{m}$ grain size). As a consequence of the length and structure of the cotton matrix, initial millings were accomplished in $5 \mathrm{~min}$ intervals at $25 \mathrm{~Hz}$. Carefully inverting the sample vial containing the cotton to mix each milled portion avoided heterogeneity related issues as well as the separation of the cotton based on milled fiber size. Triplicate samples were then massed (400. $\mathrm{mg}+/-10 \mathrm{mg}$ ) from this milled subsample.

To improve the analytical measurement of the cotton pellets an IS was added to each sample. The internal standard consisted of a liquid spike of a single element ICP grade standard, $400 \mathrm{ppm}$ scandium (RICCA, Arlington, TX, USA). Following the addition of the IS the powder was dried in a hot block for $24 \mathrm{hrs}$ at $60^{\circ} \mathrm{C}$. To further decrease the particle size of the powder and to homogenize the additional IS each sample was milled for an additional 20 minutes at $25 \mathrm{~Hz}$. The amount of mill time was optimized by comparing the influence of mill time with results obtained in analyses obtained in the measurement performed by different analytical techniques. The following variables would be used to evaluate optimum milling conditions: signal stability, precision, within pellet variation and between pellet variation. Table 25 presents the change in particle size distribution as mill time was increased for IAEA V-9, North Carolina and New Mexico cotton from 2005. These particular samples and standards were selected because they originate from different growing regions responsible for different quality cotton, Upland cotton (North Carolina) and Pima (New Mexico). American Pima cotton is characterized by extremely long fibers ${ }^{131}$. In order to thoroughly assess the applicability of the milling procedure, the extremes in fiber length that could be encountered had to be considered in the development of the sample preparation procedure. In addition to analytical comparisons of the milling times, scanning electron microscopy (SEM) was used to determine the range of particle grain size. Figure 33 shows the SEM images for the optimized mill time of 20 min. Figure 33 demonstrates that even a SRM obtained in "powder from" requires further grinding to decrease the fibrous 
nature of the powder. The use of a 20 minute homogenization step resulted in improved transient signal stability and a higher SNR of both ICP and LIBS data.

Table 25: Effect of mill time of particle size distribution of unprocessed cotton

\begin{tabular}{|c|c|c|c|}
\hline \multirow{2}{*}{ Sample } & \multirow{2}{*}{ Mill time (min.) } & \multicolumn{2}{|c|}{ Particle size $(\boldsymbol{\mu m})$} \\
\cline { 3 - 4 } & & Average & Std. deviation \\
\hline IAEA V-9 & 0 & 140 & 53 \\
\hline IAEA V-9 & 5 & 30 & 11 \\
\hline N. Carolina & 15 & 12 & 6 \\
\hline New Mexico & 15 & 15 & 8 \\
\hline N. Carolina & 20 & 13 & 2 \\
\hline New Mexico & 20 & 13 & 6 \\
\hline
\end{tabular}

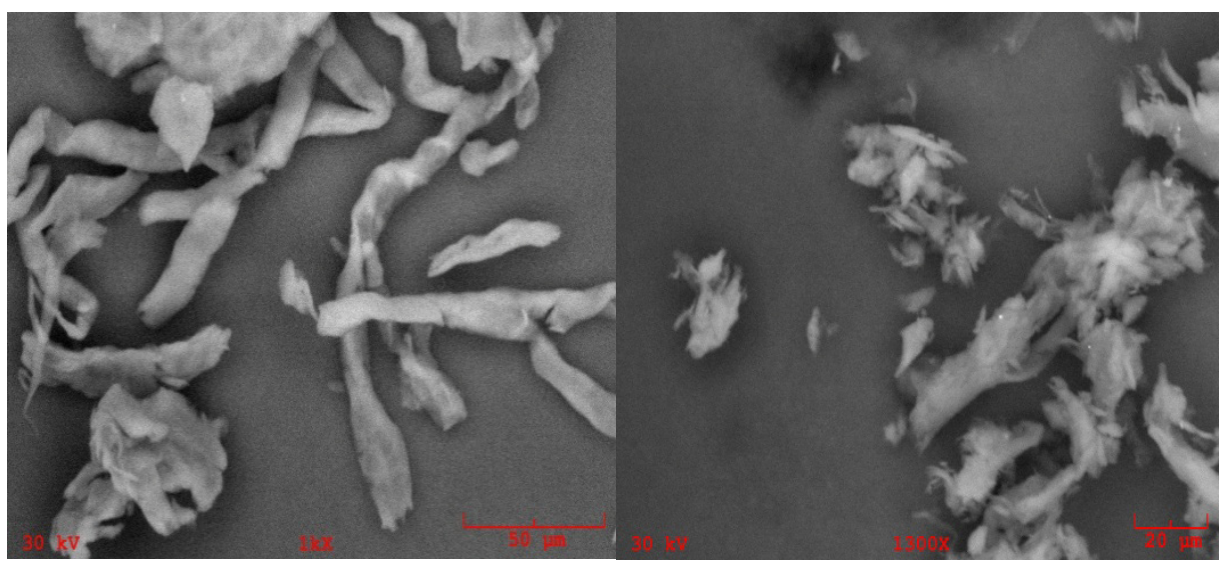

Figure 33: SEM imaging of cotton powder; IAEA V-9 powder prior to any milling (left). New Mexico cotton following 20 minutes of milling (right).

The powder (10-15 $\mu \mathrm{m}$ in size) was then pressed into a pellet using a pellet press (Carver, Wabash, IN, USA) by applying $4500 \mathrm{psi}$ in the presence of a vacuum for two min. The vacuum was used to remove unnecessary air between the powder granules, which resulted in a denser matrix, which has been reported to improve analytical analyses. Figure 34 shows the final cotton pellet, prior to analysis, $12 \mathrm{~mm}$ in diameter by $2 \mathrm{~mm}$ in thickness. The size of the pellet is advantageous in that it allowed for several analyses to be conducted on a single pellet. Analysis by all elemental analysis techniques could be conducted on a single pellet. 


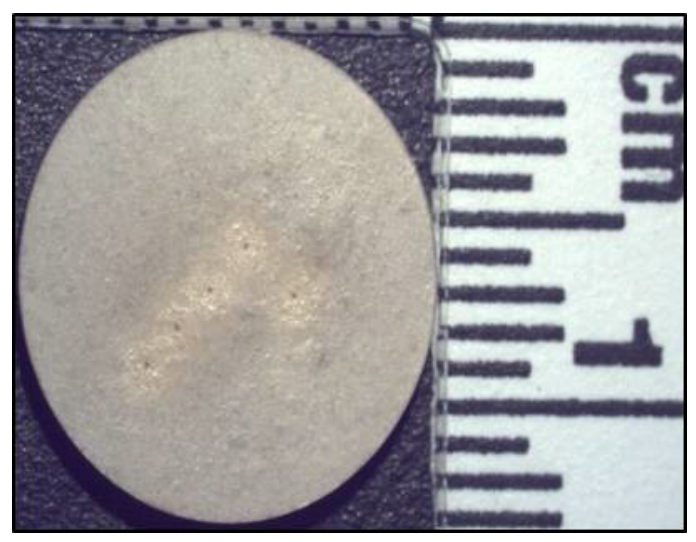

Figure 34: Unprocessed cotton pellet.

\subsubsection{Cotton SRM}

The IAEA has developed a cellulose based cotton powder, IAEA V-9, with certified metal concentrations. The SRM has been utilized in the elemental analysis of primarily cotton textiles $^{148,149}$. Table 26 contains the certified concentrations and the $95 \%$ confidence interval (C.I.) obtained from several independent laboratory analyses ${ }^{150}$. When selecting a suitable SRM, the standard ideally, should be matrix matched and have a composition and metal content similar to what is expected for the material being analyzed.

Table 26: IAEA V-9 cotton cellulose SRM composition

\begin{tabular}{|c|c|c|}
\hline Analyte $^{\mathbf{1}}$ & Concentration (ppm) & $\mathbf{9 5 \%}$ C.I. \\
\hline $\mathrm{Al}$ & 44 & $13-53$ \\
\hline $\mathrm{Ba}$ & 9 & $6-12$ \\
\hline $\mathrm{Ca}$ & 240 & $220-260$ \\
\hline $\mathrm{Cu}$ & 0.59 & $0.47-0.94$ \\
\hline $\mathrm{Fe}$ & 11 & $7-15$ \\
\hline $\mathrm{Mg}$ & 53 & $46-67$ \\
\hline $\mathrm{Mn}$ & 0.15 & $0.12-0.21$ \\
\hline $\mathrm{Na}$ & 56 & $49-64$ \\
\hline $\mathrm{Ni}$ & 0.09 & $0.07-0.2$ \\
\hline $\mathrm{Sr}$ & 0.65 & $0.54-0.96$ \\
\hline $\mathrm{V}$ & 0.09 & $0.07-0.1$ \\
\hline
\end{tabular}

${ }^{1}$ Other analytes present, however only the elements pertinent to this research are included in table 26. 
The IAEA V-9 SRM has a wide range of metal content that falls between that encountered in the cotton textile and the unprocessed commodity. The affects the capacity of using the SRM for several elements that vary greatly from the unprocessed to the processed stages of cotton textile production. On the basis of this knowledge, an "in-house" unprocessed cotton standard was developed. The IAEA-V9 was used as a secondary standard to determine the analytical validity of the resulting calibration approach.

\subsubsection{Development of an In-House Unprocessed Cotton Standard}

The North Carolina 2005 (abbr. NC2005) sample was selected as an appropriate "in-house" cotton standard on the basis of 1) sample abundance and 2) elemental profile. The typical element concentrations for elements of varying contribution to the NC2005 composition are reported by the three elemental analysis techniques employed in this research are shown in table 27. These results were obtained from the analysis of different NC2005 cotton pellets made from different batches of the fibrous milled portion. The concentrations were obtained using a standard addition approach $^{53}$ to determine concentration of an unknown, in this instance NC2005. The small standard deviation both within and between the analytical techniques not only reflects the comparability and agreement in analyses, but also that optimal sampling strategy has been achieved to account for the heterogeneity of the matrix.

Because IAEA V-9 tends to fall at one extreme - low or high - in regards to typically encountered unprocessed cotton, an additional cotton sample (Arizona 2006, AZ2006) was used as a second means of calibration verification to ensure the widespread dynamic range of the NC2005 calibration approach. The AZ2006 sample was chosen because it fell opposite in the concentration ranges as the IAEA V-9, but also because of the abundance of sample $(\sim 200 \mathrm{~g})$ available for subsequent sampling and analysis. The approximate base concentration in AZ2006 
was determined by generating several standard addition curves, similar to the approach for determining the NC2005 blank concentration.

Table 27: Baseline concentration of some analytes in NC2005 reported with different techniques

\begin{tabular}{|r|c|c|c|c|c|}
\hline \multicolumn{1}{|l|}{ Date } & Technique & Al & Ba & Mg & Sr \\
\hline 17-Apr-10 & MS & 11.5 & 1.4 & 454 & 0.6 \\
\hline 14-May-10 & LIBS & 10.1 & 2.1 & 381 & 0.8 \\
\hline 19-May-10 & MS & 8.3 & 1.1 & 422 & 0.5 \\
\hline 16-Jun-10 & LIBS & 7.6 & 1.5 & 305 & 0.7 \\
\hline 17-Jun-10 & MS & 10.6 & 2.1 & 433 & 0.5 \\
\hline 7-Jul-10 & LIBS & 7.6 & 2.5 & 375 & 0.6 \\
\hline 17-Aug-10 & MS & 10.8 & 1.5 & 480 & 0.6 \\
\hline 5-Sep-10 & OES & 11.1 & 1.4 & 343 & 0.7 \\
\hline 22-Sep-10 & MS & 8.1 & 1.2 & 413 & 0.6 \\
\hline 23-Sep-10 & LIBS & 6.8 & 2.1 & 331 & 0.7 \\
\hline 25-Sep-10 & LIBS & 7.2 & 1.0 & 330 & 0.7 \\
\hline 28-Sep-10 & LIBS & 10.5 & 1.4 & 360 & 0.8 \\
\hline & Average (ppm) & 9.2 & 1.6 & 386 & 0.7 \\
\hline & Std dev (ppm) & 2 & 0.5 & 60 & 0.1 \\
\hline
\end{tabular}

Figure 35 corresponds to sample calibration curves obtained using NC2005 as the cotton matrix. The calibration curves were generated using the developed LIBS and LA-ICP-OES method for cotton analysis.

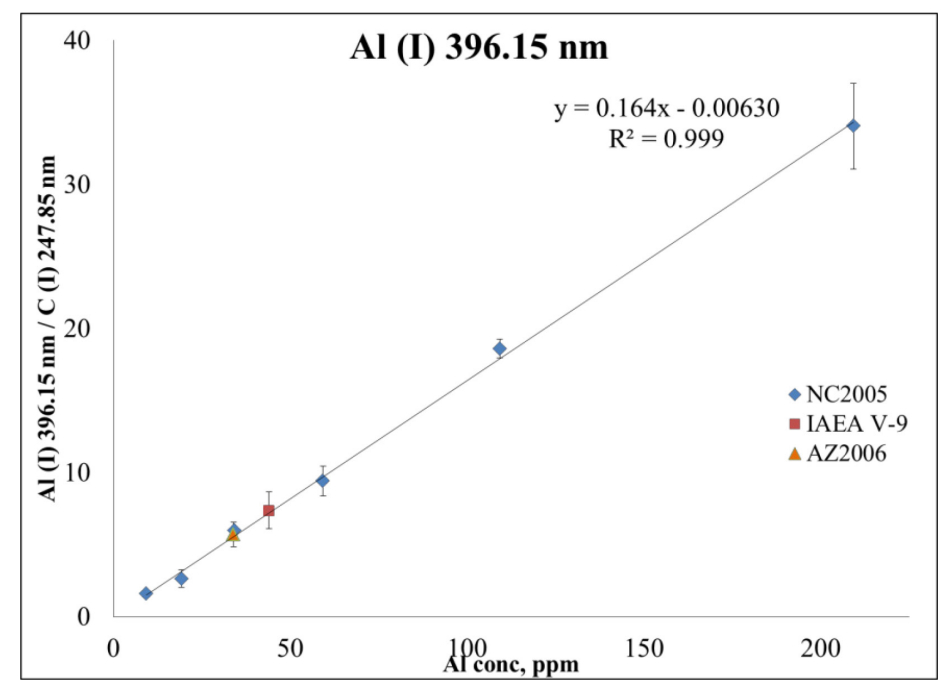

Figure 35a: NC2005 calibration curves generated with LIBS and LA-ICP-OES, LIBS example 1. 


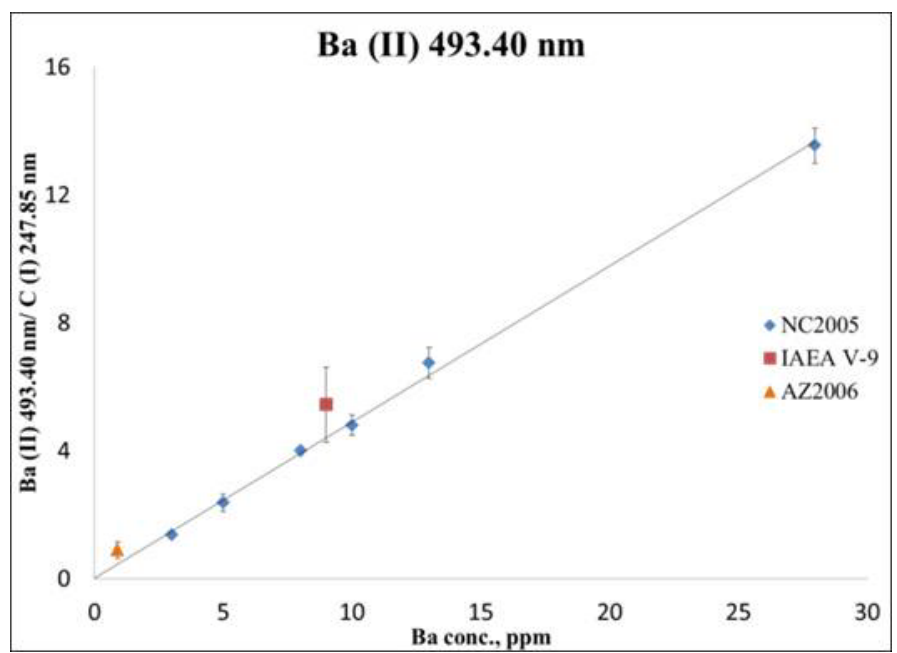

Figure 35b: NC2005 Calibration curves generated with LIBS and LA-ICP-OES, LIBS example 2.

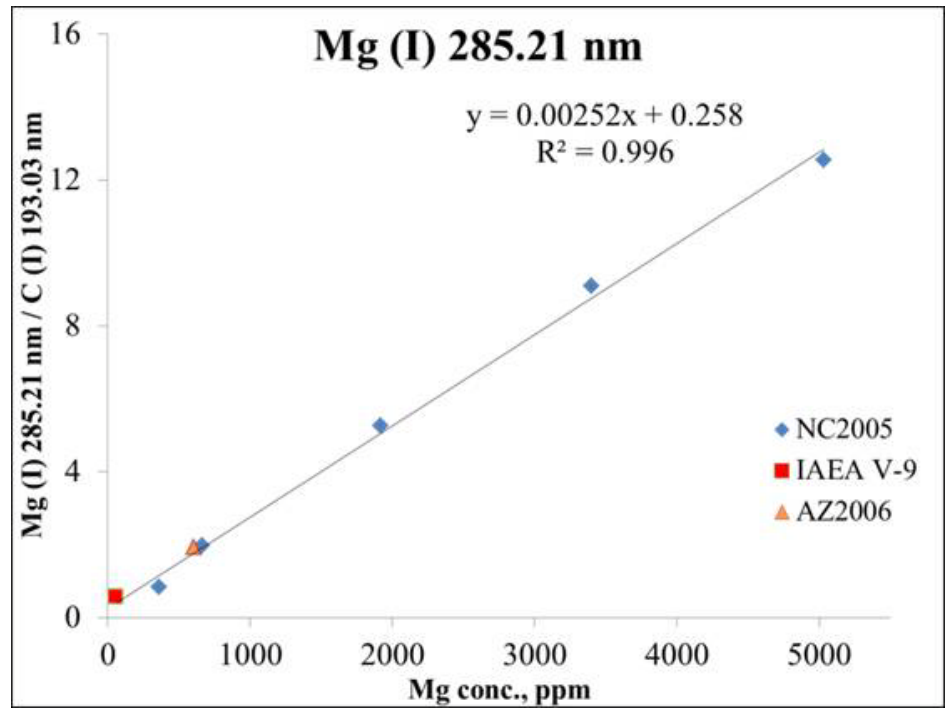

Figure 35c: NC2005 calibration curves generated with LIBS and LA-ICP-OES, LA-ICP-OES example.

Especially evident in the LIBS calibration curves is the ability of an atomic IS emission line to account in pulse-to-pulse variations and improve linearity and reproducibility of analyte emission lines of both atomic and ionic transitions. 
Atomic emission techniques, particularly LIBS, have the ability to utilize either the emission peak intensity or the peak area for data interpretation. The calibration curves presented in figure $35 \mathrm{a}-\mathrm{b}$ utilized peak intensity, however it should be noted that peak area yielded similar results.

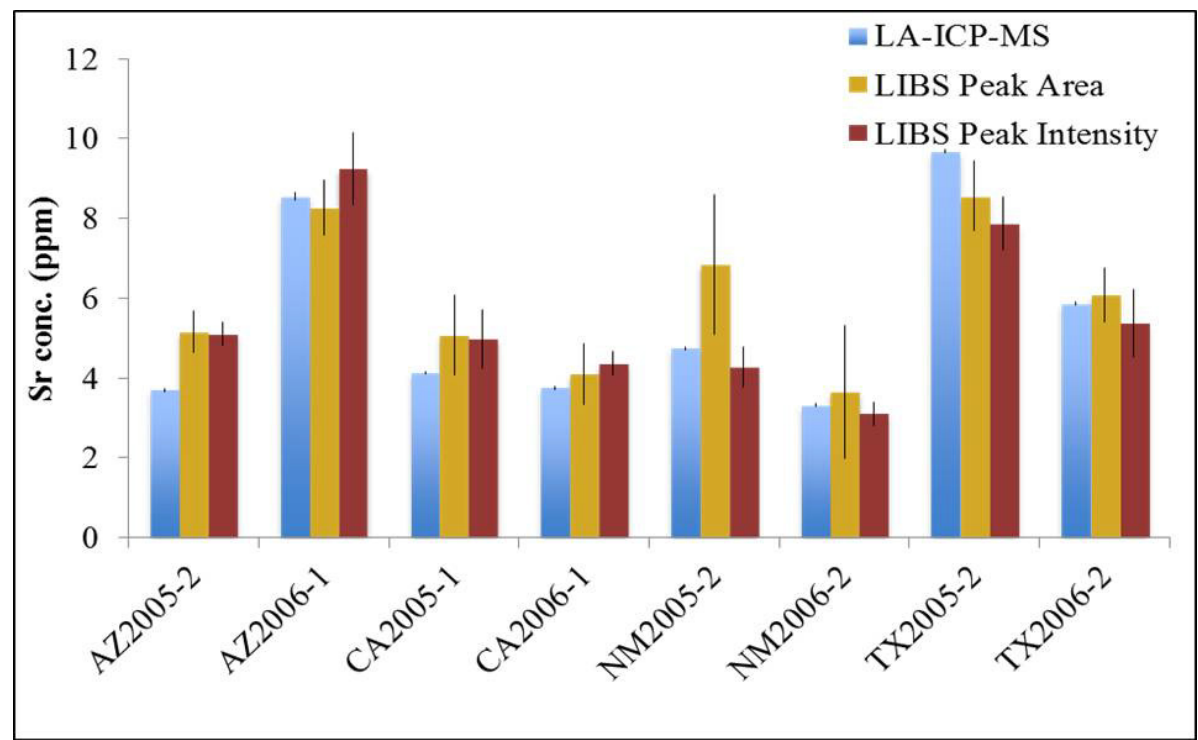

Figure 36: Comparison of concentrations obtained in LIBS using peak intensity and peak area.

\subsubsection{Description of a Test Sample Set}

The initial test sample set used to evaluate the ability to see differences in samples that pertain to a specific geographic region have been described in table 24 , previously described in section 6.3.1.1.

\subsubsection{Comparison of LA-ICP-OES and LIBS}

Both LA-ICP-OES and LIBS are atomic emission techniques. In both instances spectrometers and optics are used to collect, direct and separate the light into discrete wavelengths that correspond to atomic and ionic transitions of particular elements. The manner in which the analytical approaches differ is in the type of plasma generated. LIBS produces a transient laserinduced plasma that grows to only millimeters $(\mathrm{mm})$ in size, prior to completely decaying. On the 
other hand, LA-ICP-OES utilizes a continuous plasma (ICP) that requires a method to transport the sample or small amounts of the sample to the plasma source. A similar laser to that which is used in both of the atomic emission techniques. The output energy, and thus the fluence is much lower in order to produce particles in place of vaporizing and ionizing the ablated material.

Continuous and transient plasmas differ in composition and temporal regimes. In addition to interactions between the analyte(s), electrons and gas molecules, a continuous plasma also has a constant background emission, or background equivalence concentration (BEC) ${ }^{5}$. The concentration has to be overcome in order to detect the analyte(s). The BEC varies across the broad spectral range, resulting in a different BEC for each emission line possible. Because a LIBS plasma decays over time, background radiation in the form of continuum decays at a much faster rate than emission processes. Continuum radiation is a result of Bremsstrahlung and radiative recombination interactions. The principles behind these processes have been described in detail in section 2.2.3. The temporal depletion of this type of background allows for the detection of analytes after a time delay.

Both of these plasmas have different time regime intervals. The time regime for LIBS analysis in general, is relatively short since the rate of decay for the transient plasma relies on the amount of ejected mass and vaporized material that occurs with each individual laser pulse. In addition, a plasma produced in an ambient environment quenches the plasma, decreasing the lifetime. A continuous plasma has the advantage of a constant delivery of mass via a channel of gas. As soon as the emission decay occurs the gas flow is able to sweep it out of the plasma viewing region and replace it with new, ionized and excited material that is just starting the decay process.

Regardless of the differences in plasma properties both techniques are capable of accomplishing trace analytical analysis. This ability is both demonstrated and the analytical performance is evaluated using an unprocessed cotton matrix. 


\subsubsection{LA-ICP-OES Experimental Design}

\subsubsection{LA-ICP-OES Setup and Instrumentation}

The LA-ICP-OES setup and general instrumentation has been previously described in other chapters of this dissertation. Therefore, for the sake of brevity the information will not be repeated. The laser ablation unit used for the analysis of unprocessed was the Cetac LSX-500 (Cetac Technologies, Omaha, NE, USA). A summary of the instrumental and ablation parameters have been presented previously (section 4.3.3.1).

\subsubsection{Element Menu}

The element menu has been selected on the basis of the ability to provide the necessary sensitivity, be free of interferences, and span the dynamic range that is essential to conducting quantitative analysis. Table 28presents the element and emission line identities for the analytes and IS (carbon).

Table 28: LA-ICP-OES emission lines for unprocessed cotton analysis

\begin{tabular}{|c|c|}
\hline Element Category & Analyte \\
\hline \multirow{4}{*}{ Detectable } & $\mathrm{Cu}$ (I) $327.39 \mathrm{~nm}$ \\
\hline & $\mathrm{Fe}$ (II) $238.20 \mathrm{~nm}$ \\
\hline & $\mathrm{Fe}$ (II) $239.56 \mathrm{~nm}$ \\
\hline & Mn (II) $257.61 \mathrm{~nm}$ \\
\hline \multirow{8}{*}{ Monitored } & $\mathrm{Ba}$ (II) $493.40 \mathrm{~nm}$ \\
\hline & $\mathrm{Ca}$ (II) $315.88 \mathrm{~nm}$ \\
\hline & $\mathrm{Ca}$ (II) $317.93 \mathrm{~nm}$ \\
\hline & $\mathrm{K}$ (I) $766.49 \mathrm{~nm}$ \\
\hline & $\mathrm{Mg}$ (II) $279.55 \mathrm{~nm}$ \\
\hline & $\mathrm{Mg}$ (II) $280.21 \mathrm{~nm}$ \\
\hline & $\mathrm{Na}$ (I) $588.99 \mathrm{~nm}$ \\
\hline & $\mathrm{Sr}$ (II) $421.55 \mathrm{~nm}$ \\
\hline \multirow{7}{*}{ Element Menu } & $\mathrm{Al}$ (I) $396.15 \mathrm{~nm}$ \\
\hline & $\mathrm{Ba}$ (II) $455.40 \mathrm{~nm}$ \\
\hline & $\mathrm{Fe}$ (I) $259.93 \mathrm{~nm}$ \\
\hline & $\mathrm{Mg}$ (I) $285.21 \mathrm{~nm}$ \\
\hline & $\mathrm{Mn}$ (II) $259.37 \mathrm{~nm}$ \\
\hline & $\mathrm{Sr}$ (II) $407.77 \mathrm{~nm}$ \\
\hline & C (I) $193.03 \mathrm{~nm}$ \\
\hline
\end{tabular}




\subsubsection{LIBS Experimental Design}

\subsubsection{LIBS Setup and Instrumentation}

The LIBS setup that was utilized for the elemental analysis of unprocessed cotton for forensic application was the in-house LIBS system equipped with the IR (1064 nm) wavelength Nd:YAG laser described in section 2.3.2.3. The setup utilized the lab-built, air-gapped doublet that was described in that section. A higher output energy of $74 \mathrm{~mJ}$ (at laser head) was utilized in a bath of $\operatorname{Ar}(0.5 \mathrm{~L} / \mathrm{min})$. Although this was a higher output energy than recommended as a universal LIBS parameter, the optimization in an environment other than air can lead to different optimal parameters. However, this concept has not been explored and is outside the scope of this dissertation.

The Ar bath was delivered utilizing a "T" type connector (Thermo Scientific, Rochester, NY, USA) which split the flow into two separate gas lines. Two flows set up across from one another at the height of the cotton pellet were used to minimize disturbance in the plasma. Figure 37 depicts the setup. Side view of setup depicted in (A). Overheard view (B) demonstrates that the tubing setup supports the thorough bathing of the sample in Ar.

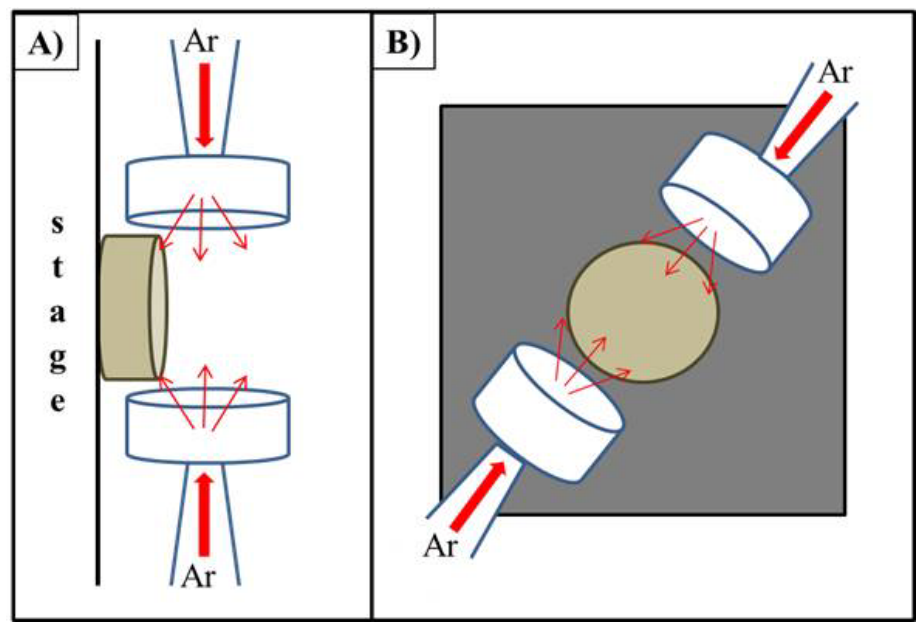

Figure 37: Setup for argon introduction in LIBS. 
Parameters utilized in this analysis have been described in table 29. The plasma light was collected at a $90^{\circ}$ incident to the laser light and was focused onto a $50 \mu \mathrm{m}$ FOC.

Table 29: LIBS parameters for cotton analysis

\begin{tabular}{|c|c|}
\hline Parameter & Operating Condition \\
\hline Laser Conditions (1064 nm, Nd:YAG) \\
\hline Laser Power & $74 \mathrm{~mJ}$ at head \\
\hline LTSD & $0.7 \mathrm{~mm}$ \\
\hline Ablation mode & Spot \\
\hline Number of shots & 90 shots, $6 \mathrm{cleaning}$ \\
\hline Frequency & $0.66 \mathrm{~Hz}$ \\
\hline Spectrometer Conditions \\
\hline Gate delay & $2.0 \mu \mathrm{s}$ \\
\hline Gate width & $50.0 \mu \mathrm{s}$ \\
\hline Gain & 150 \\
\hline
\end{tabular}

To ensure that the setup was in proper working order a signal threshold had to be achieved using NIST1411as a performance standard. The same parameters were utilized every day. To exhibit the day-to-day variability observed, peak performance of several elements over several days is shown in figure $38 \mathrm{a}$ and the performance an emission line present in the glass SRM that is used in cotton analysis, figure $38 \mathrm{~b}$.

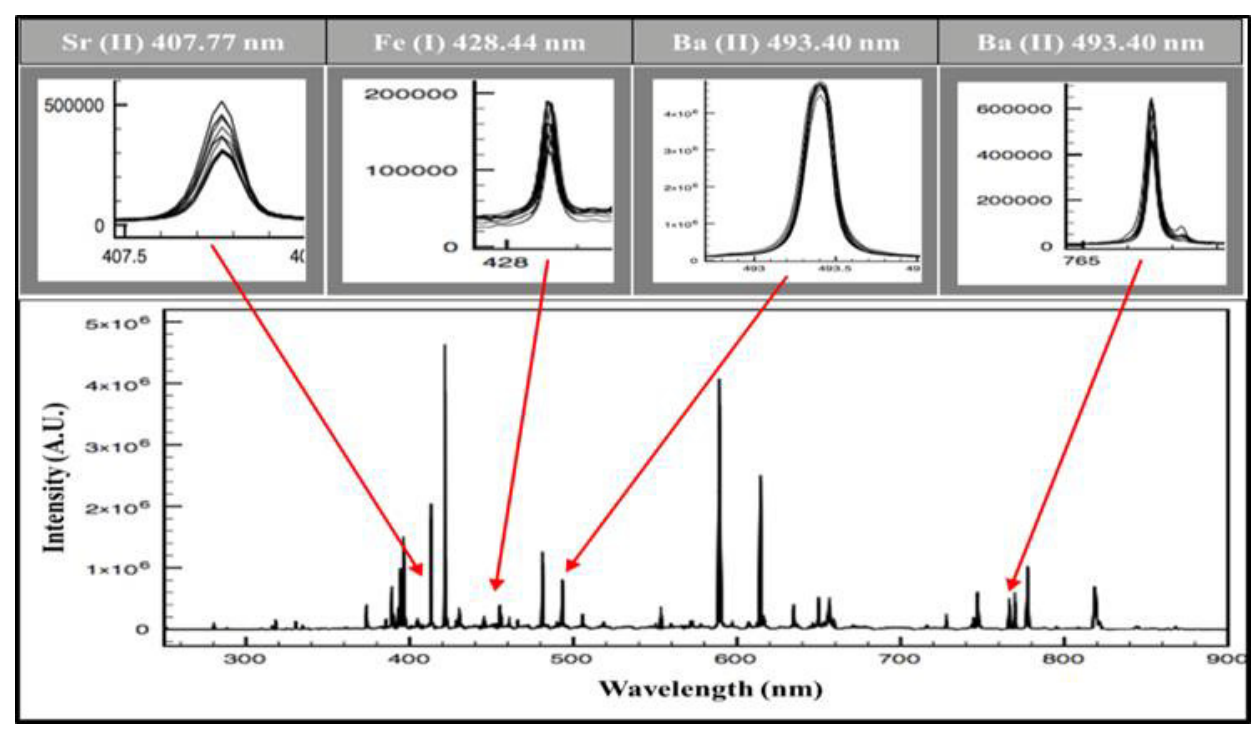

Figure 38a: SRM NIST 1411 performance using 1064 nm LIBS. 


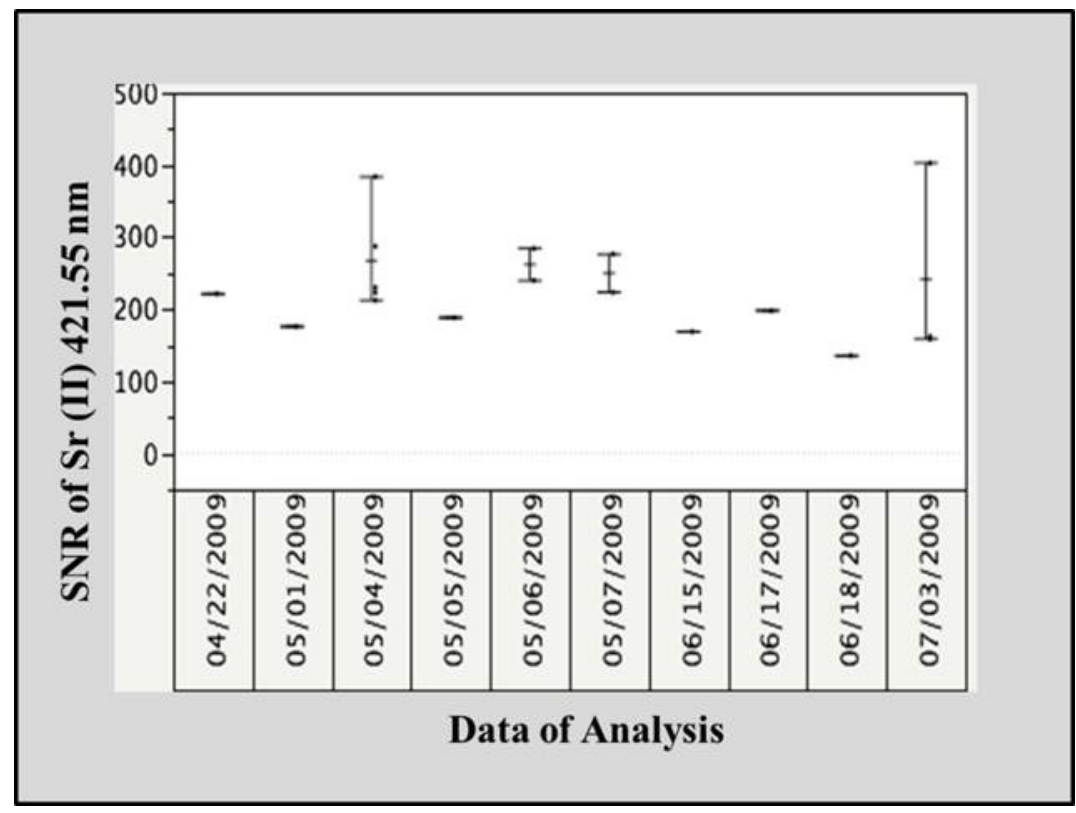

Figure 38b: Day-to-day sensitivity obtained with LIBS using SRM NIST 1411.

An overall intensity of approximately 8000 a.u. was required for a single shot, 4 x $10^{6}$ for a 20 shot accumulation. NIST 1411 was used to establish the performance of the LIBS setup, utilizing the SRM to confirm that sensitivity thresholds had been achieved prior to sample analysis.

\subsubsection{Role of Shadowgraphy in LIBS Optimization}

Shadowgraphy is the study of the shockwave produced following the generation of a transient plasma produced following a laser pulse. The magnitude in the force of the plasma is actually able to disturb the surrounding environment, yielding a wave extending out in all directions away from the sight of plasma formation. To visualize the shockwave, a second laser in the visible wavelength range, typically $400-700 \mathrm{~nm}$, is aligned perpendicular to the LIBS excitation source. A 532 nm Brilliant B (Quantel USA, Big Sky, MO, USA) laser was used for all shadowgraphy experiments. The second laser beam is not focused; rather, it is expanded and collimated so as to intersect the focal point of the laser used to conduct the LIBS experiment. The second laser, operating at a high frequency ( $20 \mathrm{~Hz}$ for example), is used as a strobe to visualize the shockwave. 
A camera, with several filters meant to reduce the intensity of the laser light, is set up opposite the collimated beam of the second laser. In order to visualize the progression of the shockwave on the ns timescale, both lasers and camera recording must be integrated using a digital delay generator. The shadowgraphy configuration used for these assessments is depicted in figure 39. In order to be able to compare the resulting shadowgraphic images to what would be encountered during an analytical LIBS experiment the $532 \mathrm{~nm}$ strobe laser and camera were aligned orthogonal to the actual LIBS setup used for all analytical measurements. In figure 39 the red beam correspond to the excitation laser, while the green beam is consistent with the shadowgraphy strobe laser

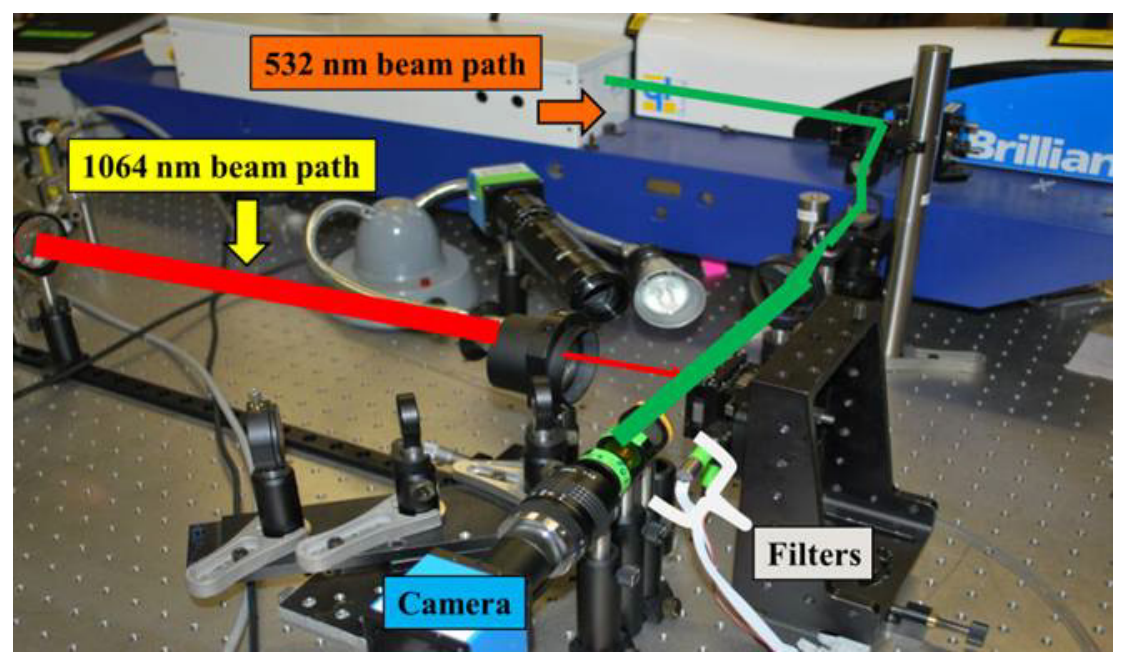

Figure 39: Shadowgraphy setup.

Shadowgraphy studies are usually conducted using a high laser frequency ${ }^{151}$. However, such a high frequency has the potential to mimic a double pulse LIBS experiment to produce a larger, longer lasting plasma accomplished with a reheating pulse ${ }^{8}$ than what would be observed with a slower frequency. To avoid this, a temporal scheme was developed to accommodate the two different extremes in frequency required. The shadowgraphy studies were conducted at the same frequency as used in analytical LIBS analysis, $0.67 \mathrm{~Hz}$. The Brilliant B laser has some internal operating conditions that presented some difficulty in the application of external timing devices. Internal constraint required that the flashlamp be run at $10 \mathrm{~Hz}$ or faster. Both the q-switch and the 
flashlamp of the $532 \mathrm{~nm}$ laser were externally triggered to meet these restrictions. To be able to accomplish shadowgraphy at this laser frequency $(0.67 \mathrm{~Hz})$ is significant because most, if not all reportings of shadowgraphy in the LIBS literature utilize a much higher operating frequency. In addition, this work addresses the applicability of shadowgraphy conducted at this frequency.

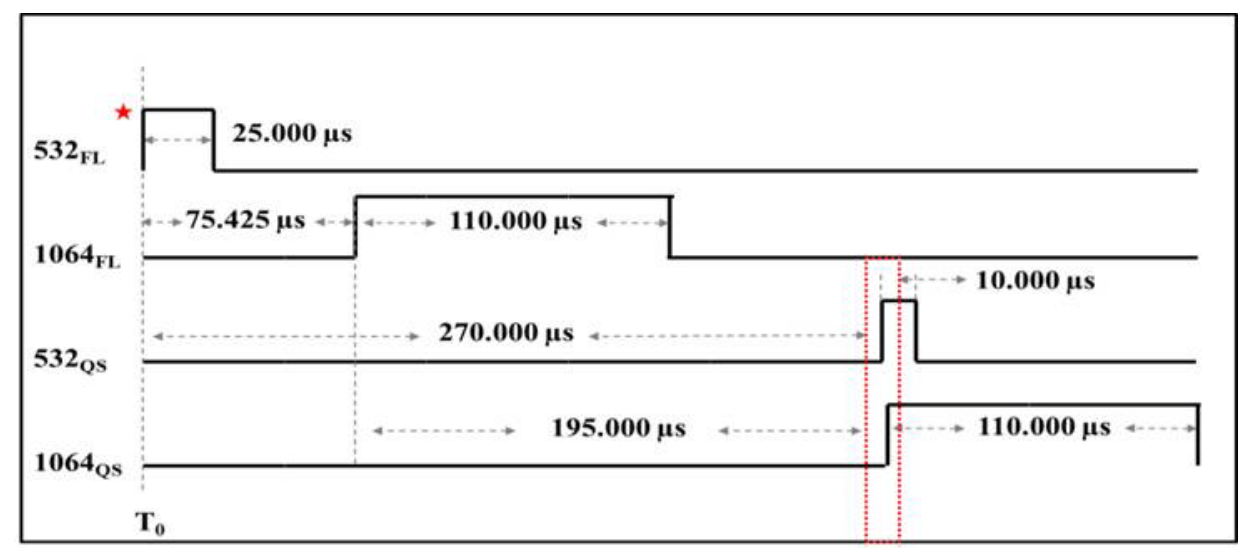

Figure 40a: Flashlamp and q-switch timing for shadowgraphy.

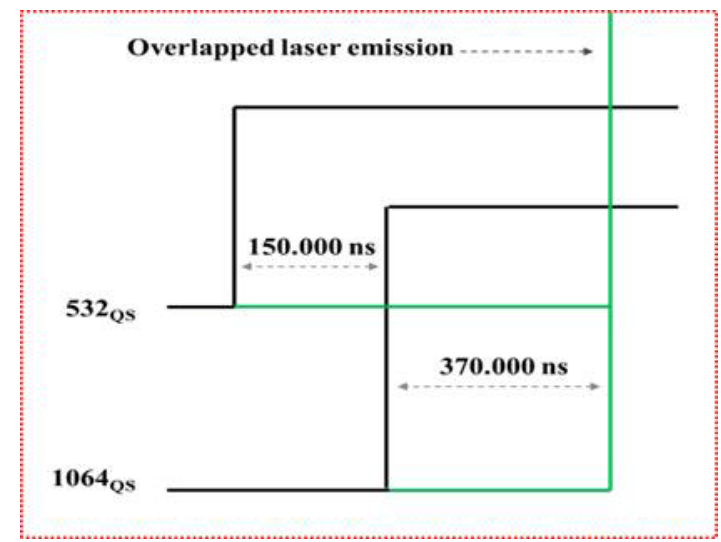

Figure 40b: Enlarged q-switch region.

To accommodate the internal programming of the $532 \mathrm{~nm}$ laser while maintaining a lower operating frequency of the $1064 \mathrm{~nm}$ laser $(0.67 \mathrm{~Hz})$ and also using a single 4-channel delay generator the 1064 timing and sync options had to be cycled. External pulses triggering the 1064 nm flashlamp and q-switch were only delivered to the laser one every 15 number of pulses in order to achieve a laser frequency of $0.66 \mathrm{~Hz}$ or a repetition rate of $1.5 \mathrm{~s}$. Since the q-switch is 
synchronized to the flashlamp this is accomplished by increasing the delay of the flashlamp from $\mathrm{T}_{\mathrm{o}}$ (denoted by the red star in figure 40a). The timing of the camera was synchronized to the 532 nm operating conditions, This results in a difference in time between the release of the emission of the two lasers, i.e. increasing the delay of the $532 \mathrm{~nm}$ flashlamp to $500 \mu \mathrm{s}$ implies that the image captured by the camera pertains to a shockwave $500 \mu$ s after the laser pulse. There is some electrical jitter encountered so the additive uncertainty in all the timing parameters is $50-75 \mathrm{~ns}$. Although the flashlamp was controlled internally, the electrical signal to initiate the firing sequence was given by the delay generator; ensuring that the two lasers, being controlled both by internal and external means are synchonized.

Figure $40 \mathrm{~b}$ provides an example of the timing delays required so that the two pulses overlap - the plasma $\left(\mathrm{t}_{\mathrm{o}}\right)$. To visualize the shockwave later in time after the actual pulse, the timing of the 532 $\mathrm{nm}$ setup is stepped further from the overall $\left(\mathrm{T}_{0}\right)$.

It has been established that the overall size and morphology of the shockwave is indicative of the plasma formation and expansion, and thus can be used to make inferences regarding the location and shape of the plasma to a point ${ }^{14}$. After expansion to approximately $1 \mathrm{~mm}$, the shockwave detaches from the plume which does not expand beyond this dimension ${ }^{14}$. Shadowgraphy has been employed in the current studies to evaluate the effectiveness of the LIBS configuration in different environments (air and Ar). The exploration of plasma morphology was essential to determine if the general alignment of the FOC was relevant to both of the LIBS environments. In addition, differences in laser-sample interactions can be inferred from plasma size and morphology. This was also taken in to consideration in part because of the variation in color of cotton pellets. Samples of different colors have the potential to absorb laser power differently, which could lead to inconsistency in analytical results if not prevented or accounted for and can be evaluated, in one respect, by assessment of the plasma morphology. 
Although the remainder of this section is dedicated to interpreting shadowgraphy results, the inclusion of this information is more appropriate in this section since it addresses important steps in method development and optimization.

The shockwave morphology was first assessed in the absence of a matrix in both air and Ar environments. Figure 41 depicts the shockwaves observed in the environments using the same time scale. At a $900 \mathrm{~ns}$ delay the shockwave was approximately $1 \mathrm{~mm}$. The size reflects that which has been reported in the literature ${ }^{14}$.
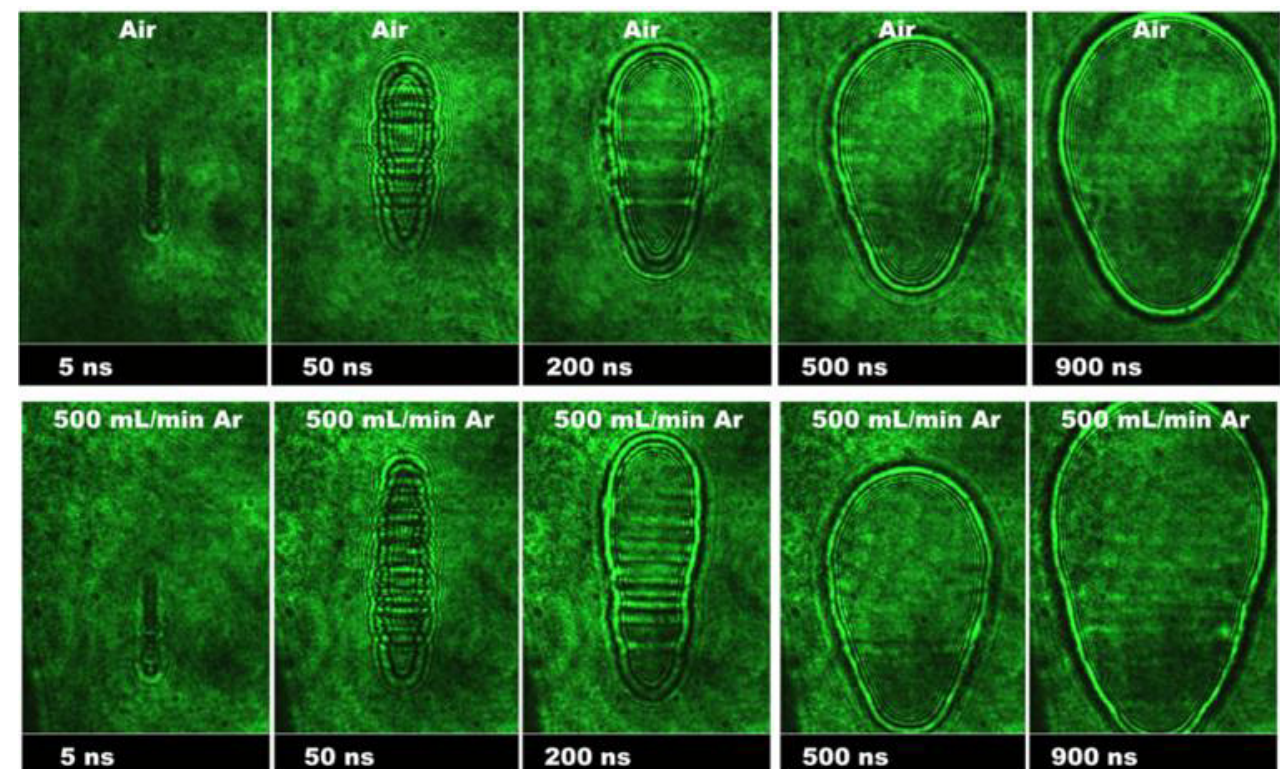

Figure 41: 1064 nm LIBS shockwave obtained in different environments.

Homogeneity of the plasma morphology is observed as time from $t_{0}$ increases. Plasmas in both environments are approximately the same size at $900 \mathrm{~ns}$. Early development in the plasma morphology and plasma shape varies, which is apparent in the elongation of the Ar plasma before $500 \mathrm{~ns}$; this is because plasmas produced in Ar tend to expand in the direction from which the excitation laser originated ${ }^{14}$. The difference in sharpness and detail in the plasma edges of the shockwaves can be attributed to the differences in density of the surrounding environment. However, in both instances the persistence of several small microplasmas expanding out in 
sequence decreases at $900 \mathrm{~ns}$, suggesting a more homogenous environment within the confined space at the time in which LIBS related emission is collected.

When a matrix is introduced, in figure 42 , similarities are observed although the plasma is broader in an argon environment in the presence of a matrix. The Ar plasma on the target surface expands outward in all directions more so than the shockwave in air, which demonstrates a tendency to expand directly up from the surface. Although the spatial distribution varies, the plasma center in both environments are similar, the highest point from surface is nearly identical.
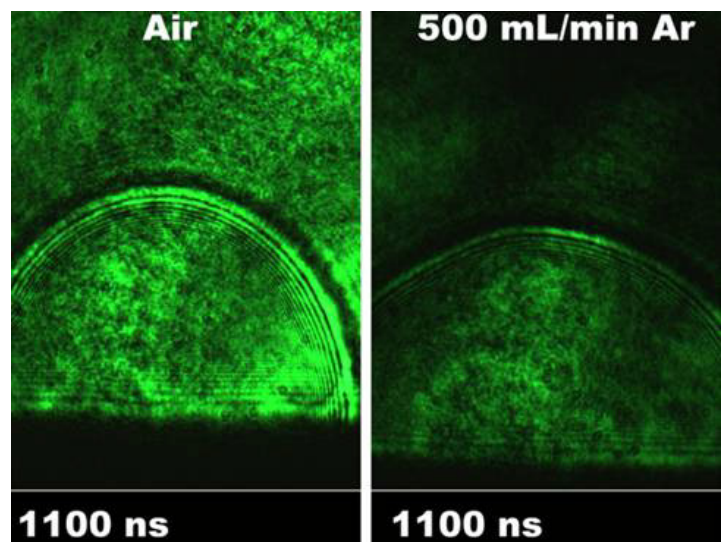

Figure 42: 1064 nm LIBS shockwave obtained following ablation of unprocessed cotton.

From this, it can be concluded that at the time in which plasma light is being collected, the LIBS microplasma is similar in size and location for both environments. Though there may be differences in composition, which will be addressed later.

The similarity reflects the versatility of the LIBS configuration and allows comparisons between the two environments to be made with only minor FOC adjustment. Because the emission collection optics employed in this research collects all the light produced during LIBS excitation, the similarity in plasma center is important to ensuring that the FOC can be easily centered on the emission in both instances, which addresses the flexibility of the instrument for conducting various analytical measurements. 
To evaluate the development of the cotton crater from a different view than what has been presented in chapter 2 (aspect ratios and ablation efficiency) the development of the plasma as repetitive laser shots were applied to the same location was also compared. Figure 43 confirms that up until the optimized number of laser shots (90shots) the plasma expansion is similar. This is important to ensure that the plasma expansion is occurring at or above the sample surface, allowing for subsequent detection.

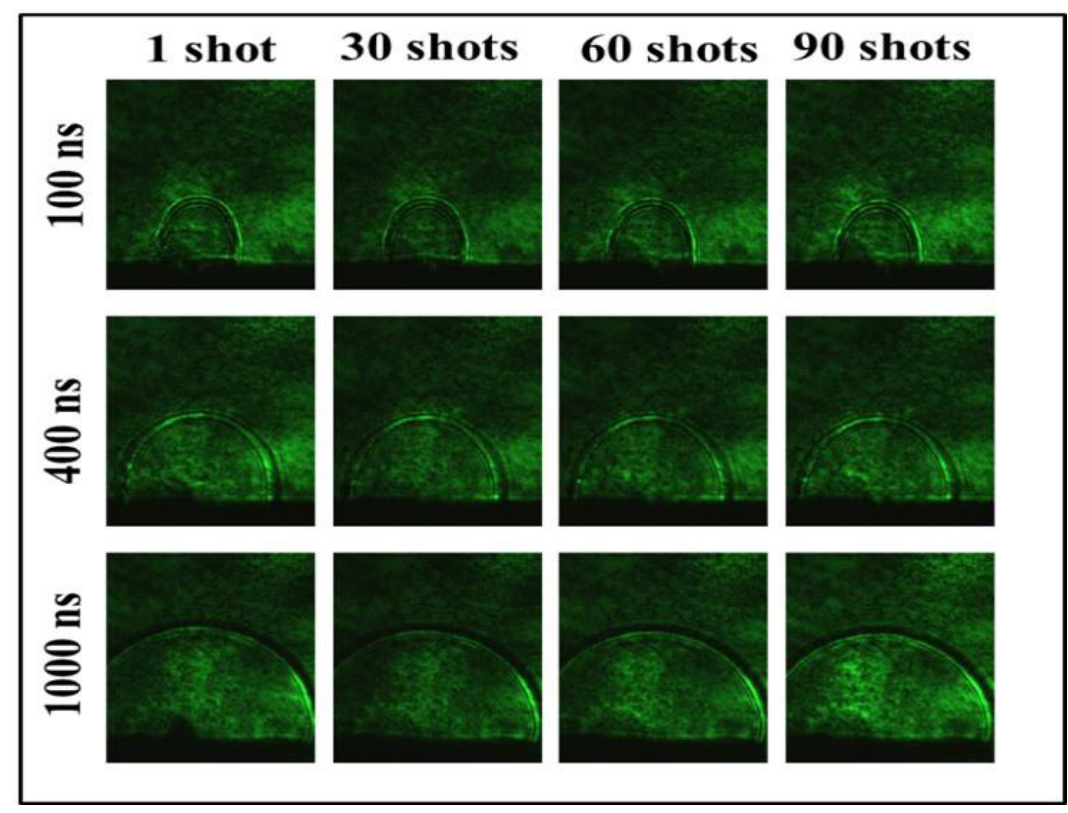

Figure 43: 1064 nm LIBS shockwave morphology as cotton crater depth increases.

For a single sample, shown in figure 43, shockwave and thus plasma consistency is observed. However, analytical implications arise if laser-sample interactions produce plasmas of varying magnitude. This was not observed within a matrix (cotton), demonstrated by three shockwaves NC2005, NM2006 and IAEA V-9 - all at $1 \mu \mathrm{s}$, yielding similar morphological properties. The pellets varied from white to a dark tan. Differences are encountered for different matrices, which is presented in figure 44 by visual inspection of the shockwave produced following soil ablation (PACS-2) in comparison to cotton. The similarity in laser-sample interaction within a single matrix is corroborated by the principles developed in chapter 2 of this dissertation. The three 
cotton samples yield aspect ratios of $0.53 \pm 0.02,0.49 \pm 0.03$ and $0.55 \pm 0.02=$, respectively. The results serve as an additional method to substantiate the previously explored concept of uniformity within matrix when making comparisons regarding several different measurements that pertain to LIBS analysis.

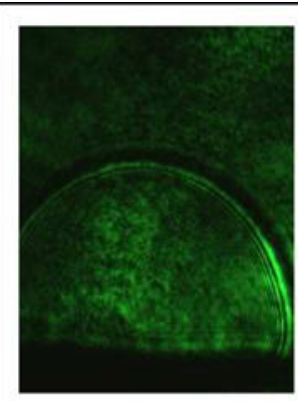

NC2005

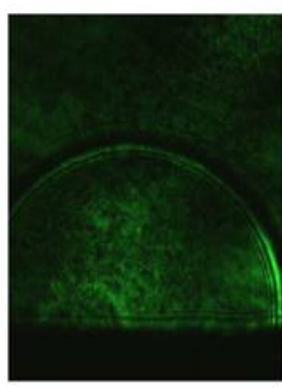

NM2006

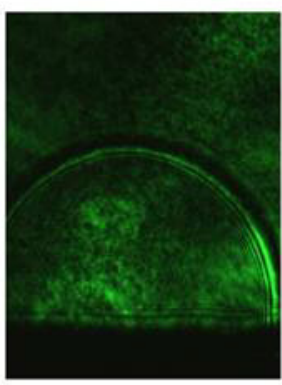

IAEA V-9

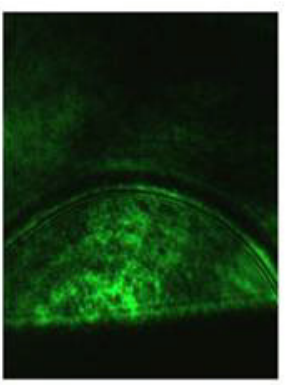

PACS-2

Figure 44: Comparison of 1064 nm LIBS shockwaves for pellets of different color.

The similarity in morphology between the different colored cotton samples confirms that the LIBS setup and FOC alignment is conducive to alignment of the different samples using the optimized conditions. The information obtained using shadowgraphy and the experiments conducted in chapter 2 aided in the development of an analytical LIBS method for unprocessed cotton that is capable of accomplishing quantitative trace analysis.

\subsubsection{Element Menu}

Elements selected for the LIBS analysis of unprocessed cotton pellets met the same criteria described for LA-ICP-OES. Table 30 presents the LIBS element menu and other emission lines detected and monitored using this method. Carbon was utilized as an IS for this technique as well. 
Table 30: LIBS emission lines for unprocessed cotton analysis

\begin{tabular}{|c|c|}
\hline Element Category & Analyte \\
\hline \multirow{4}{*}{ Detectable } & $\mathrm{Ca}$ (II) $315.88 \mathrm{~nm}$ \\
\cline { 2 - 2 } & $\mathrm{Ca}$ (II) $317.93 \mathrm{~nm}$ \\
\hline \multirow{4}{*}{ Monitored } & $\mathrm{K}$ (I) $766.49 \mathrm{~nm}$ \\
\cline { 2 - 2 } & $\mathrm{Mg}$ (I) $285.21 \mathrm{~nm}$ \\
\cline { 2 - 2 } & $\mathrm{Mg}$ (II) $279.77 \mathrm{~nm}$ \\
\cline { 2 - 2 } & $\mathrm{Na}$ (I) $588.99 \mathrm{~nm}$ \\
\cline { 2 - 2 } & $\mathrm{Na}$ (I) $589.59 \mathrm{~nm}$ \\
\hline \multirow{5}{*}{ Element menu } & $\mathrm{Al}$ (I) $396.15 \mathrm{~nm}$ \\
\cline { 2 - 2 } & $\mathrm{Ba}$ (II) $493.40 \mathrm{~nm}$ \\
\cline { 2 - 2 } & $\mathrm{Fe}$ (I) $428.44 \mathrm{~nm}$ \\
\cline { 2 - 2 } & $\mathrm{Mg}$ (II) $280.27 \mathrm{~nm}$ \\
\cline { 2 - 2 } & $\mathrm{Mn}$ (II) $279.44 \mathrm{~nm}$ \\
\cline { 2 - 2 } & $\mathrm{Sr}$ (II) $421.55 \mathrm{~nm}$ \\
\cline { 2 - 2 } & $\mathrm{C}$ (I) $247.85 \mathrm{~nm}$ \\
\hline
\end{tabular}

\subsection{Results and Data Analysis}

\subsubsection{Analytical Figures of Merit}

\subsubsection{LIBS Analytical Figures of Merit}

Measures of performance using the LIBS method for cotton analysis are presented for IAEA-V9 and the secondary in-house calibration standard AZ2006 in tables 31 and 32, respectively. The values have been obtained over several analyses conducted over approximately three months so as to address the long-term stability The LIBS method is capable of performing quantitative analysis for elements of low (IAEA V-9) and high (AZ2006) concentrations that may be encountered in the elemental analysis of unprocessed cotton. Bias values are $<15 \%$ for most analytes in each standard with the exception for analytes close to the detection limit. For example, the high bias is encountered for Mn in IAEA V-9 is attributed to the low concentration in the SRM. This emission line is close to a sensitive Mg peak (Mg (II) $279.77 \mathrm{~nm}$ ). The close proximity of the peaks makes it difficult to differentiate from the shoulder of the $\mathrm{Mg}$ emission 
line. The performance of sensitivity improves significantly for AZ2006, which has a Mn concentration within the encountered sample range.

Table 31: Analytical performance of LIBS using IAEA V-9

\begin{tabular}{|c|c|c|c|}
\hline Analyte & Avg Concentration, ppm & \%RSD & \% Bias \\
\hline Al & 44 & 6.9 & - \\
\hline Ba & 9 & 4.5 & - \\
\hline Fe & 15 & 12.3 & 37 \\
\hline Mg & 51 & 4.1 & -3.7 \\
\hline Mn & 0.25 & 9.5 & 67 \\
\hline Sr & 0.75 & 6.7 & 15 \\
\hline
\end{tabular}

Table 32: Analytical performance of LIBS using AZ2006

\begin{tabular}{|c|c|c|c|}
\hline Analyte & Avg Concentration, ppm & \%RSD & \% Bias \\
\hline Al & 35 & 4.7 & 2.9 \\
\hline Ba & 2 & 6.2 & 120 \\
\hline Fe & 39 & 8.1 & 4.0 \\
\hline Mg & 700 & 22.2 & -17. \\
\hline $\mathbf{M n}$ & 4.9 & 5.4 & 2.1 \\
\hline Sr & 5.8 & 20.5 & 22. \\
\hline
\end{tabular}

In addition to the quantitative analysis of unprocessed cotton, the sensitivity of the methods must be addressed and compared. The LODs for the LIBS analysis are depicted in table 33. The LODs have been calculated using the approach outlined in equation 16 and 17 of this dissertation. Along with the LODs, the ranges of concentrations obtained in the test set are also included. The concentrations have been determined using LA-ICP-MS because of the sensitivity of the technique.

Table 33: LIBS LODs for cotton analysis

\begin{tabular}{|c|c|c|}
\hline Analyte & LOD (ppm) & Sample Range \\
\hline $\mathrm{Al}$ & 7.4 & $3.9-64$ \\
\hline $\mathrm{Ba}$ & 0.9 & $1-5$ \\
\hline $\mathrm{Fe}$ & 3.3 & $2-90$ \\
\hline $\mathrm{Mg}$ & 10. & $300-910$ \\
\hline $\mathrm{Mn}$ & 0.17 & $1.1-6.9$ \\
\hline $\mathrm{Sr}$ & 0.30 & $0.20-10$. \\
\hline
\end{tabular}


As depicted in table 33, LIBS does not have the sensitivity to detect all trace elements, the Sr LOD falling within the concentration range determined. The samples that were below the detection limit were all from the same area of the U.S. - Mississippi. The use of Sr in the element menu was not dismissed. Presence and absence could still be a useful tool in establishing geographic origin and will be further addressed using statistical evaluation. This technique is capable of achieving quantitative analysis with good precision and bias for most analytes presented for both cotton standards of varying concentration implying that LIBS is capable of conducting analysis of samples with precision and accuracy.

\subsubsection{LA-ICP-OES Analytical Figures of Merit}

The quantitative ability of LA-ICP-OES was also evaluated using the same two standards as previously presented. Tables 34 and 35 correspond to the bias and precision achieved for IAEA V-9 and AZ2006, respectively. The values for the cotton SRM and the in-house standard have been obtained over several days of analysis conducted over approximately three months.

Table 34: Analytical performance of LA-ICP-OES using IAEA V-9

\begin{tabular}{|c|c|c|c|}
\hline Analyte & Avg Concentration, ppm & \%RSD & \% Bias \\
\hline $\mathrm{Al}$ & 43 & 4.0 & -2.2 \\
\hline $\mathrm{Ba}$ & 11 & 1.5 & 22 \\
\hline $\mathrm{Fe}$ & 12 & 1.4 & 9.0 \\
\hline $\mathrm{Mg}$ & 50 & 1.7 & -5.7 \\
\hline $\mathrm{Mn}$ & 0.18 & 11.5 & 22 \\
\hline $\mathrm{Sr}$ & 0.71 & 5.6 & 9.2 \\
\hline
\end{tabular}

Table 35: Analytical performance of LA-ICP-OES using AZ2006

\begin{tabular}{|c|c|c|c|}
\hline Analyte & Avg Concentration, ppm & \%RSD & \% Bias \\
\hline $\mathrm{Al}$ & 31 & 4.0 & -8.8 \\
\hline $\mathrm{Ba}$ & 0.9 & 0.8 & - \\
\hline $\mathrm{Fe}$ & 20 & 1.4 & -47 \\
\hline $\mathrm{Mg}$ & 620 & 0.6 & 2.5 \\
\hline $\mathrm{Mn}$ & 4.5 & 2.9 & 2.3 \\
\hline $\mathrm{Sr}$ & 5.1 & 0.8 & 13 \\
\hline
\end{tabular}


Tables 34 and 35 highlight the analytical capabilities of LA-ICP-OES, demonstrating precision and bias that exceeds that of LIBS when considering the same standards. Improvements in some instances might be the result of emission line selection. For example, the bias and precision of both Fe and Mn improves greatly when emission lines lower in the UV range are used. The current spectrometer used in LIBS is unable to perform in this region due to the drop-off in quantum efficiency. Although LA-ICP-OES has pre-programmed emission lines suggesting instrumentation that is not flexible to the needs of the analyst the ability to perform in the deep UV is beyond the reaches of some LIBS spectrometers.

Sensitivity, and thus applicability of the method was addressed using LOD. Table 36 presents the LODs achieved with this technique. The LOD for all analytes has been determined using the approach outlined in equations 16 and 17 of this dissertation. In comparison to the observed range in sample concentration LA-ICP-OES does not have any limitations to the samples or analytes that can be detected when the selected element menu is used. The sensitivity of the method in conjuncture with the ability to achieve quantitative analysis make LA-ICP-OES a good analytical tool for conducting elemental analysis.

Table 36: LA-ICP-OES LODs for cotton analysis

\begin{tabular}{|c|c|c|}
\hline Analyte & LOD (ppm) & Sample Range \\
\hline $\mathrm{Al}$ & 0.52 & $3.9-64$ \\
\hline $\mathrm{Ba}$ & 0.3 & $1-5$ \\
\hline $\mathrm{Fe}$ & 0.20 & $2-90$ \\
\hline $\mathrm{Mg}$ & 0.20 & $300-910$ \\
\hline $\mathrm{Mn}$ & 0.11 & $1.1-6.9$ \\
\hline $\mathrm{Sr}$ & 0.090 & $0.20-10$. \\
\hline
\end{tabular}

\subsubsection{Comparison of Analytical Abilities}

While both LA-ICP-OES and LIBS have demonstrated the ability to perform quantitative analysis of unprocessed cotton the analytical performance of the instrumental approaches was performed. 
Both techniques are capable of achieving short-term stability, as demonstrated by the small variation in the day-to-day assessments of sensitivity documented for each respective technique in QC charts presented in this and previous chapters. However, the variations in \%RSD obtained with IAEA V-9 and AZ2006 address the ability to achieve long-term stability. The use of LAICP-OES demonstrates good precision over three months for both the low and high concentration standard, whereas performance of LIBS deteriorates over time for analytes of varying concentrations. However, when the concentration of analyte in either standard is in middle of the sample concentration range, the performance of LIBS is relatively good. The variation in precision though, may affect the ability to generate a long-term model for geographic region predictions of the unprocessed cotton. In general, the short and long-term stability of LA-ICPOES is more amenable to this application.

The LODs for LA-ICP-OES are lower than that which could be achieved with LIBS analysis in an Ar environment. However, a comparison of absolute detection limits presents a direct comparison of detection capabilities by taking into account the mass removed and subsequently utilized in the analysis. Absolute detection limits are calculated on the basis of the mass removed to produce a crater in each respective technique. Figures 45 and 46 depict the craters that results for laser ablation (fig 45) and LIBS (fig 46). Mass removal results in $9.9 \mu \mathrm{g}$ and $33.3 \mu \mathrm{g}$ for laser ablation and LIBS, respectively.

Analytical LIBS requires three times more mass to conduct the analysis than is necessary for LAICP-OES, which results in different absolute detection limits, table 37, for each technique. While both techniques may suffer from incomplete ionization and so all mass removed may not contribute to detectable emission, nonetheless the mass is removed during the analysis. 


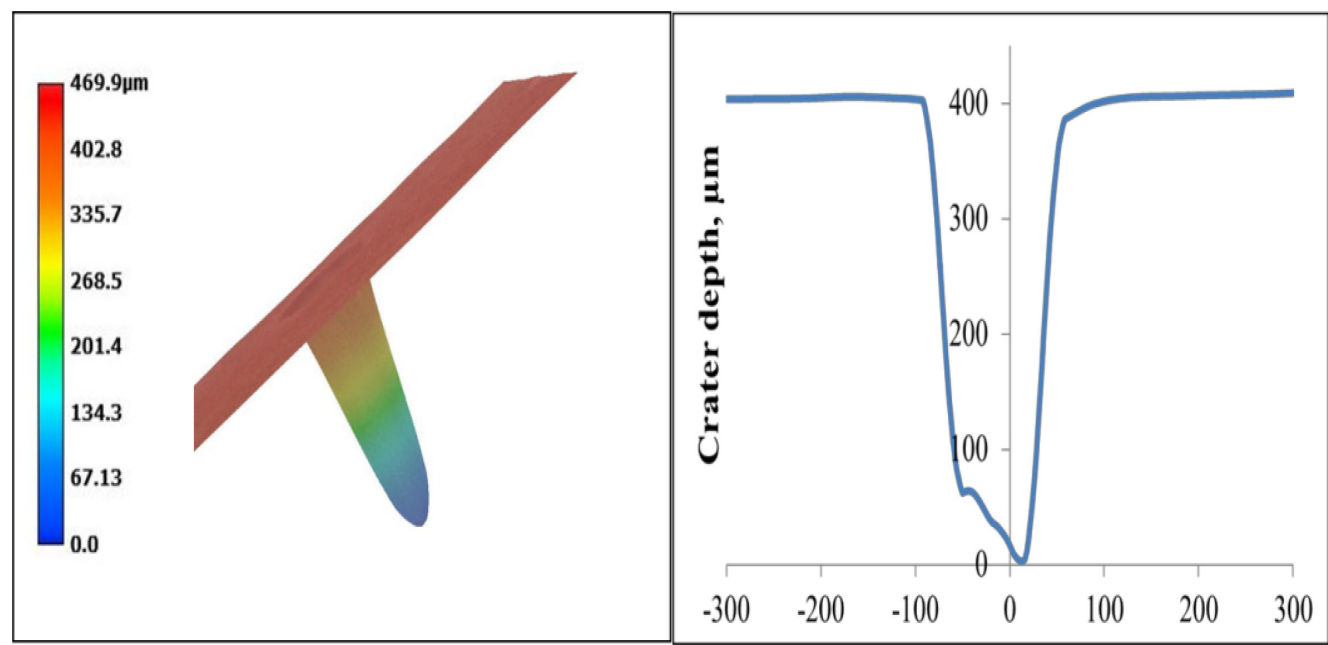

Figure 45: Typical LA-ICP-OES cotton crater; 3D cotton crater obtained with LA-ICP-OES (left). Side slice of the crater taken at its deepest point (right).
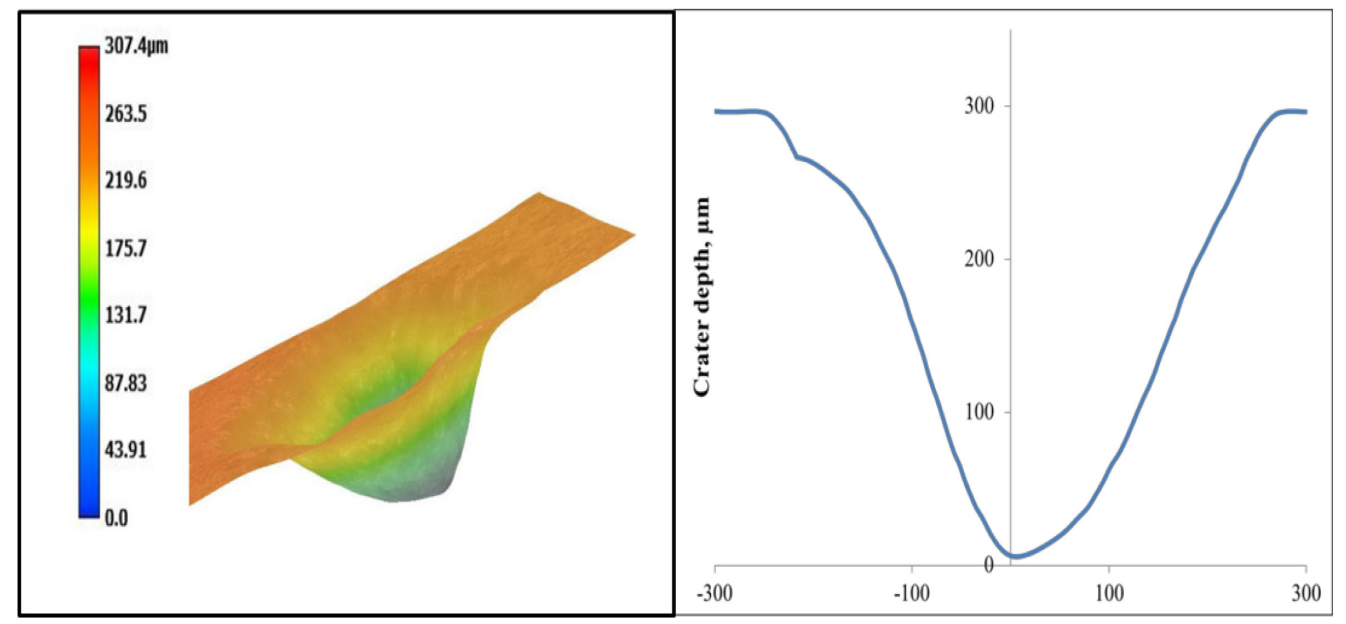

Figure 46: Typical LIBS cotton crater; 3D cotton crater obtained with LIBS (left). Side slice of the crater taken at its deepest point (right).

Table 37: Absolute detection limits for LA-ICP-OES and LIBS analysis of unprocessed cotton

\begin{tabular}{|c|c|c|}
\hline Analyte & LA-ICP-OES (pg) & LIBS (pg) \\
\hline $\mathrm{Al}$ & 5.0 & 250 \\
\hline $\mathrm{Ba}$ & 3 & 30 \\
\hline $\mathrm{Fe}$ & 20 & 110 \\
\hline $\mathrm{Mg}$ & 30 & 330 \\
\hline $\mathrm{Mn}$ & 0.90 & 6.1 \\
\hline $\mathrm{Sr}$ & 0.3 & 10. \\
\hline
\end{tabular}


Analytical measurement conducted using LA-ICP-OES can be accomplished with better sensitivity and performance than LIBS while consuming less mass. However, a full comparison of the two techniques should also include the ability to detect differences in the sample set.

\subsubsection{Performance of a Sample Set}

To assess the actual applicability of each technique to detecting not only differences between samples, but particularly differences that are associated with geographic source or region of origin. A complete statistical evaluation of all sample sets and techniques will be presented later. The focus of this assessment is strictly visual identification of the variation represented in the samples as determined by each respective technique. Further comparisons are not made because of the small size of the sample set. A classification scheme will be presented in a later chapter dedicated to model prediction.

Principal components analysis (PCA) is a multivariate chemometric tool that can reduce the complexity of the data sets. When several analytes are used in data interpretation it is often necessary to reduce the complexity in order to visually represent the data. This is especially true when the ultimate goal is to display the data as a plot. PCA is not a data reduction technique. It is,

however, a variable reduction technique ${ }^{152}$. PCA reconstructs the data set in the form of orthogonal linear combinations. The linear combinations are referred to as principal components (PCs).

The number of PCs is much less than the number of variables, i.e. a variable reduction. Several PCs can be used to reconstruct the data set. However with each additional component the amount of variation accounted for decreases. The most variation is represented by the first component and becomes successively less with each additional component. 
The use of PCA can be further explained using eigenvalues and eigenvectors. The names of these terms correspond to their role: an eigenvalue is a single number associated with each principal component, while an eigenvector consists of several numbers comprising a vector ${ }^{153}$. Eigenvectors and eigenvalues are presented in pairs. The eigenvector with the largest corresponding eigenvalue is considered the primary $\mathrm{PC}$, and so on ${ }^{153}$. Each analyte contributes to every eigenvector, however this contribution is not equal nor proportionate. Analytes may contribute to the variation accounted for in one eigenvector more so than in another. This information can be used to determine the extent to which the variation in the data set can be associated with particular analytes and therefore can be used to confirm or dismiss the use of particular variables.

The resulting graphical representation of the PCA is referred to as a score plot. Each sample possesses as many scores as principal components have been maintained. A score is a value that represents the influence the related eigenvector has on the particular sample. The scores are then plotted on a $2 \mathrm{D}$ or $3 \mathrm{D}$ plot. The variation represented by each score, which correspond to the eigenvalue, is cumulative. The plot then, if in $2 \mathrm{D}$, represents the variation accounted for in eigenvalue one and thus eigenvector one as well as the second pair of eigenvalues and eigenvectors. In the 2D comparison or $\mathrm{x}-\mathrm{y}$ plot, separation of samples on the basis of variation can be determined using quadrants. If a vertical or horizontal line originating from the zero point on that particular axis, or if a diagonal line through $\mathrm{y}=\mathrm{x}$ can separate samples it can be considered that a significant distance exists between the data to suggest the presence of groups within the data. Score plots will be presented here.

\subsubsection{Comparison Utilizing PCA}

Figure 47 corresponds to the PCA of the LIBS test sample set. The PCA accounts for $85.1 \%$ of the variation in the data set (PC1: $52.6 \%$ and $\mathrm{PC} 2: 32.5 \%)$. To aide in trend visualization the data 
points have been colored according to two regions of the U.S. cotton belt, the southeastern U.S. and the southwestern U.S. Since this test set is fairly abridged in comparison to the actual cotton belt, the identification of "southeastern U.S." is used to distinguish North Carolina and Mississippi from Arizona, California, New Mexico and Texas (southwestern U.S.). While some separation between these two classifications is observed, overlap is also evident. The overlap in the plot could be due to several factors: 1) the abridged sample set may not be representative of the variation present in cotton that originates from different geographic regions or 2) the selection of the element menu may not contain information relevant to geographic region. These two factors are both important to the eventual modeling of the elemental variation in unprocessed cotton as it pertains to geographic region.

To emphasize the significance of these stipulations, figure 48 presents the same LIBS data utilizing a slightly different element menu. In place of $\mathrm{Mg}$ (II) $280.21 \mathrm{~nm}, \mathrm{Mg}$ (I) $518.36 \mathrm{~nm}$ was employed and an additional Ca (II) $315.88 \mathrm{~nm}$ emission line was used in place of $\mathrm{Mn}$ (I) 279.44 $\mathrm{nm}$. The different element menu proved to be more applicable to this particular test set. The difference is particularly evident in the contribution of $\mathrm{Mg}$. If the elements were to be ranked in order according to the influence in the eigenvector associated with PC $1, \mathrm{Mg}$ is last. However, applying the same ranking for the PCA depicted in figure $48, \mathrm{Mg}$ is the most significant contribution to assessing the variation in the sample set. This is most likely because $\mathrm{Mg}$ (II) $280.21 \mathrm{~nm}$ is the product of strong emission process, and may not be as susceptible to small changes in concentration as a weaker emission line. Although, on the basis of the results of these two different PCAs one would easily and logically conclude that the second element menu more accurately depicts variations attributed to geographic region. However, when additional samples from other regions and crop years were considered, the first element menu demonstrated a more widespread applicability to accomplish geographic provenancing on a larger scale (i.e., several samples sets or samples for more areas within the U.S.). The difference in performances with 
each analyte menu stresses the importance of identifying the ultimate goal and thinking in the long term, rather than short term study performance. Ultimately the intention of this research is to achieve forecasting potential in regard to geographic provenancing of this commodity. The ability to apply what we have learned through this research exploration of the elemental profile of unprocessed cotton and the temporal and spatial variation would provide a stable modeling scheme significant to the forensic science community.

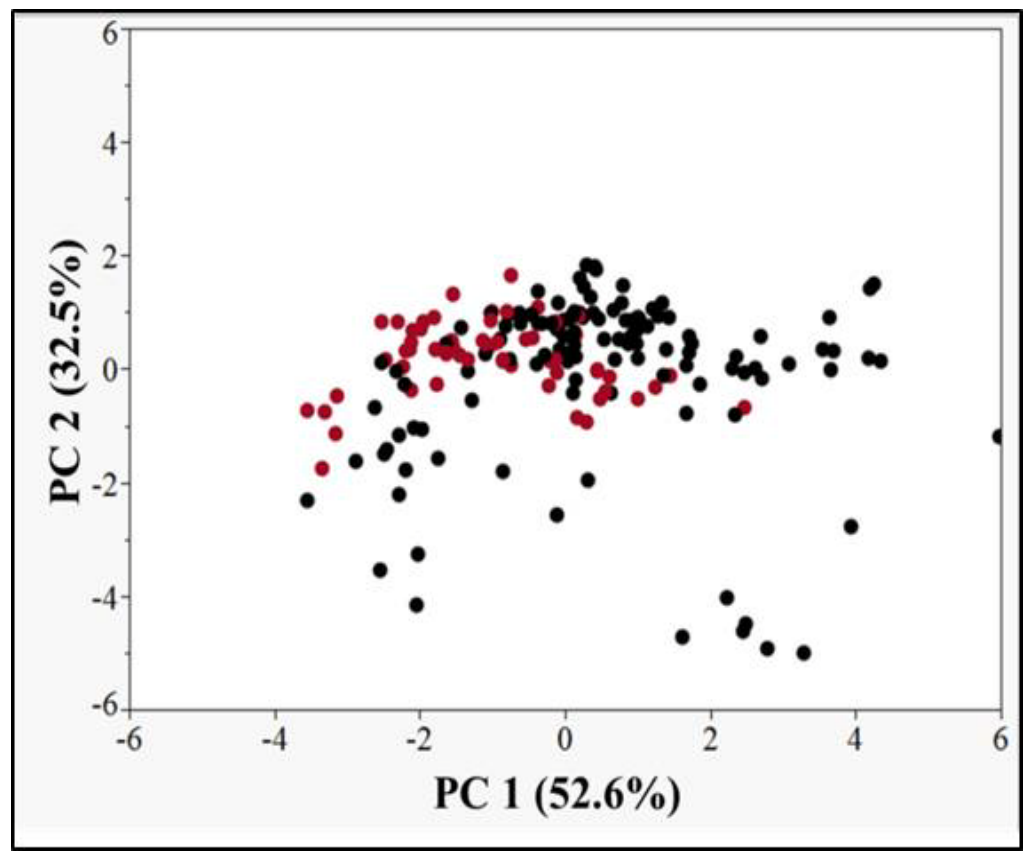

Figure 47: LIBS PCA of cotton test sample set, red = southeast U.S., black= southwest U.S.

In addition, the PCAs of the LIBS data accounted for different cumulative amounts of variation in the test sample set, with the "better" element menu (when only this study is considered) attributing to less variation, but yielding more separation between the two regions. This attests to the difficulty in comparing results strictly using visual tools for the variable reduction of complex data and for ease in pattern recognition. The use of PCA, in this research, is solely to determine if variation in the elemental profiles exists between samples and if so, to then determine if the 
variation is associated with particular geographic regions. The information is then used to determine the applicability of the techniques in question.

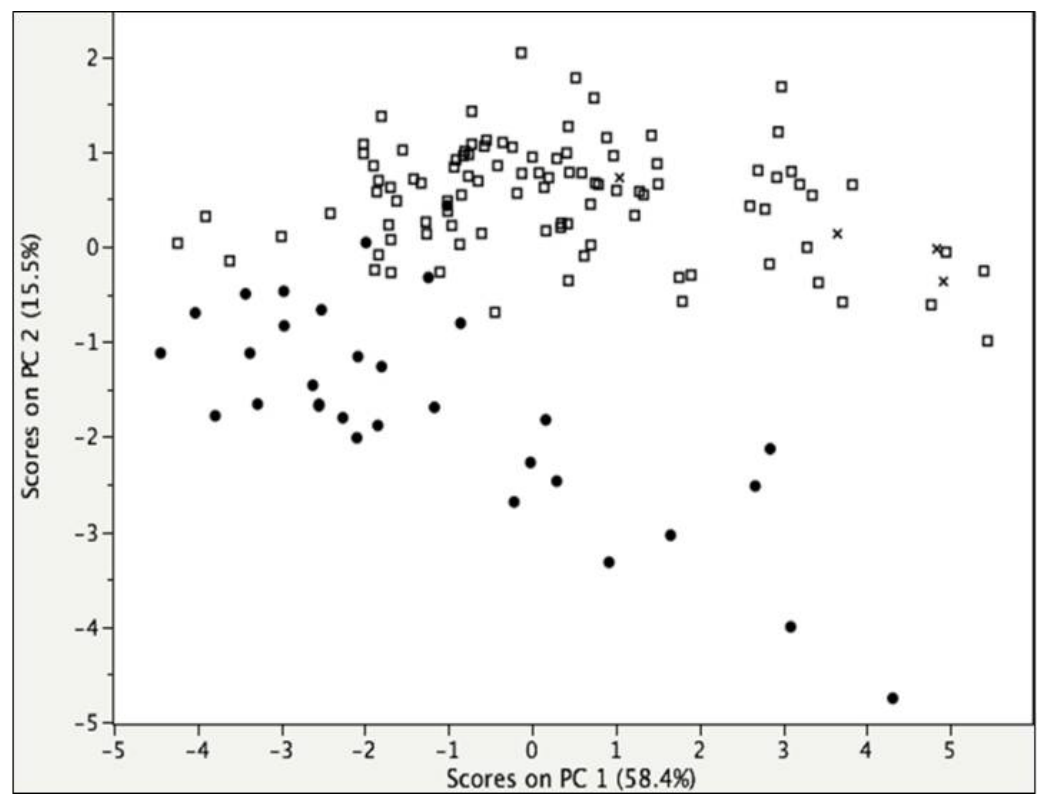

Figure 48: LIBS PCA of cotton test sample set - emphasis on emission line selection, black $=$ southeast, open squares $=$ southwest.

As a final measure of comparing the two atomic emission spectroscopic techniques, the ability of LIBS to detect differences in elemental composition was compared to that obtained with LA-ICPOES. Figure 49 depicts the PCA scores for the test set following LA-ICP-OES analysis.

However, whereas the LIBS set was run in triplicate, LA-ICP-OES analysis was only conducted in duplicate for each sample, yielding less data points for comparison; trends, however are still present.

Although the variation represented in the score plot is less than that of the LIBS data, the ease of visual recognition of the two different regions suggests that while the element menu is the same for both techniques ( $\mathrm{Al}, \mathrm{Ba}, \mathrm{Fe}, \mathrm{Mg}, \mathrm{Mn}$ and $\mathrm{Sr}$ ) the difference in emission line selection is clearly critical. 
When considering the differences in score plots between the two techniques, LA-ICP-OES is capable of providing more information regarding geographic differences for this study when the current element menus are used. At this point, for small-scale analysis, LA-ICP-OES delivers more valuable analytical information.

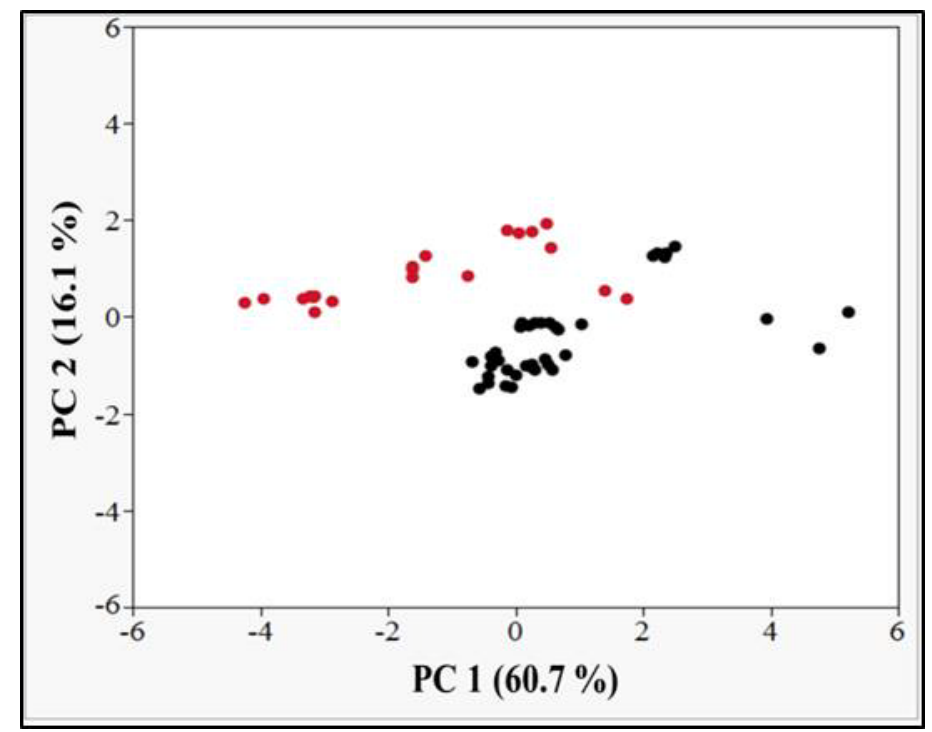

Figure 49: LA-ICP-OES PCA of cotton test sample set, red = southeast, black = southwest.

\subsection{Discussion and Conclusions}

Atomic emission spectroscopy techniques were compared for the elemental analysis of unprocessed cotton for the purpose of geographic provenancing. Both techniques demonstrated applicability, however the analytical performance and ability to detect geographic differences of LA-ICP-OES surpasses the capabilities presented by LIBS.

Less mass for detection is required by LA-ICP-OES which can be advantageous if minimal sampling, i.e. single fiber analysis is considered in the future. In addition, the preprogrammed emission line selection, is not a significant limitation to method development. A variety of emission lines that extend into the deep UV are useful to achieving sensitive analysis. The detection system of LA-ICP-OES proved to be an asset. 
In addition both techniques demonstrated relatively good short and long-term reproducibility which is important when the eventual goal is to make predictions on the basis of element concentration. Good reproducibility leads to more accurate predictions for geographic provenancing.

Both LA-ICP-OES and LIBS were able to utilize an in-house cotton standard to conduct the quantitation. This not only validates the performance of NC2005, but also the sampling strategy that was presented in this chapter. This research was able to utilize a cotton standard to accomplish quantitative analysis of unprocessed cotton and detect differences on the basis of different crop location. This is an important tool for forensic investigations and lays a thorough foundation for employing atomic emission spectroscopy techniques to conduct this and similar analysis.

\subsection{COMPARISON OF ATOMIC EMISSION SPECTROSCOPY TO LA-ICP-MS}

\subsection{Overview}

In order to evaluate alternative approaches to elemental analysis it is customary to make comparisons of analytical measures to assess the differences in performance that each respective technique is capable of producing. This chapter includes the comparison of the already introduced LIBS and LA-ICP-OES methods to LA-ICP-MS analysis of cotton. In spite of drawbacks including costly vacuum pump maintenance and the necessity of a highly skilled operator, LAICP-MS offers excellent analytical performance and is considered the elemental analysis "gold standard"

The focus of the present chapter is to determine what, if any, difference in analyte detection between inorganic spectrometry and atomic emission spectroscopy provides when attempting to accomplish geographic provenancing. 


\subsection{Introduction}

\subsubsection{Elemental Analysis Techniques}

There are several elemental analysis techniques available. These include neutron activation analysis (NAA), flame or furnace AAS, SEM/EDS, XRF, ICP techniques and LIBS to name a few. The principle analytical capabilities of several of the techniques have been discussed in 5.2.2. The rationale for selecting a particular technique relies on several requirements. The strengths and limitations of each technique must be weighed when determining which analytical approach is most appropriate. The purpose of the application and the performance requirements must be known prior to selecting the method of analysis and detection.

The criteria for instrument selection can be split into instrumental design and capabilities, analytical performance and sample related factors. Table 38 presents some of these important aspects. In addition an example emphasizing the importance of the parameter is also presented. 
Table 38: Important aspects and performance requirements considered for instrument selection

\begin{tabular}{|c|c|}
\hline Criteria & Example \\
\hline \multicolumn{2}{|l|}{ Instrumental Design } \\
\hline Capital cost & $\begin{array}{c}\text { Cost of instrument and maintenance, influence of } \\
\text { laboratory budget }\end{array}$ \\
\hline Ease of use & $\begin{array}{l}\text { End user skill set required for operation and } \\
\text { maintenance }\end{array}$ \\
\hline Flexibility & $\begin{array}{c}\text { Multi-element vs. single element, applicable to } \\
\text { several different matrices, ability to perform } \\
\text { unattended }\end{array}$ \\
\hline Instrument requirements & Gas needs, space needs, ability to dissipate heat \\
\hline \multicolumn{2}{|l|}{ Analytical Performance } \\
\hline Short-term stability & $\begin{array}{l}\text { Signal change should be less than } 2 \% / \text { hour, } \\
\text { comparability of measurements made same day }\end{array}$ \\
\hline Long-term stability & $\begin{array}{l}\text { Reproducibility of instruments, comparability of } \\
\text { measurements made over time, ability to generate } \\
\text { databases and accomplish modeling }\end{array}$ \\
\hline Sensitivity & $\begin{array}{l}\text { Analytical capability of the technique, amount of } \\
\text { sample needed to perform a single replicate }\end{array}$ \\
\hline Dynamic range & $\begin{array}{l}\text { Suitable for a broad range in sample concentration } \\
\text { within and between matrices }\end{array}$ \\
\hline Interferences & $\begin{array}{l}\text { The number of chemical or spectral interferences } \\
\text { that can hinder the applicability of the technique }\end{array}$ \\
\hline \multicolumn{2}{|l|}{ Sample Related } \\
\hline State of the sample & Solution vs. solid sampling \\
\hline Sample preparation & $\begin{array}{l}\text { Caustic digestion vs. direct analysis of solid, or } \\
\text { analysis of solid pellet }\end{array}$ \\
\hline Sample size & $\begin{array}{c}\text { Ability to analyze small samples, ability to analyze } \\
\text { irregularly shaped samples }\end{array}$ \\
\hline
\end{tabular}

The criteria listed above are applied to the comparison of the three elemental analysis techniques considered within the scope of this dissertation.

\subsection{Materials and Methods}

\subsubsection{Principles of LA-ICP-MS}

Much of the principles and concepts that pertain to LA-ICP-MS have been previously presented, as they also pertain to other techniques. The theory of atoms, the process of ionization, the workings of an ICP and the principles of laser ablation have been described in detail earlier in this dissertation so they will not be reiterated. The focus, then, will be on the detection principles of 
inorganic mass spectroscopy as they apply to inorganic MS that utilizes a continuous source plasma for ionization.

An element consists of a single type of atom. Every atom is composed of electrons, protons and neutrons. There are instances in which an atom of the same element can have a different mass. The difference in mass is the result of a different number of neutrons. The atoms of different mass are referred to as isotopes of the element. Isotopes have the same chemical properties of that element. Elements can be monoisotopic, or possess several isotopes. When there are several isotopes of a single element, each isotope exists at a particular abundance.

To be detected by inorganic mass spectrometry the isotopes must be available as ions. Ions are generated in the same manner as they are generated in ICP-OES - bombardment was excess external energy ${ }^{4}$. The degree of ionization varies, but can and has been calculated using the Saha equation $^{154}$. In general, as ionization potential increase the degree of ionization (\%) decreases.

The front-end is the same for both ICP techniques used in the current studies. The two ICP techniques are the same up until the interface that bridges the atmospheric pressure region of the ICP with the high vacuum region of the MS. The interface consists of two nickel (Ni) or platinum (Pt) cones of slightly different shaped orifices in the cones are used to direct the beam of ions towards the mass spectrometer. Following the interface a photon stop is placed in the ion beam to stop both photons and neutrals from reaching the mass spectrometer. This reduces the background signal resulting in a lower BEC for the instrument. In addition a negatively charged ion lenses is used to attract the positive ions and further attenuating the beam, while keeping it collimated.

Employing mass spectrometry as a means of detection is, in the simplest terms can be described as using a mass filter to sort and detect the analyte ${ }^{154}$. It separates the ion beam according to differences in mass, or more specifically mass-to-charge $(\mathrm{m} \backslash \mathrm{z})$. There are several types of mass 
spectrometers available to fit different analytical needs. This particular research utilized a quadrupole mass analyzer. A quadrupole, as the name implies, consists of four rods, configured as two parallel pairs. Direct and alternating current is applied to the pairs so that they oscillate completely out of phase. Ions will be deflected into the rods unless they have a specific $\mathrm{m} \backslash \mathrm{z}$ which allows them to travel the length of the rods to be detected. By adjusting the voltages, different $m \backslash z$ values can travel this path. A mass spectrometer that utilizes a quadrupole has the advantage of a simple instrumental design that offers sufficient sensitivity for most applications. A simultaneous dual system consisting of a pulse and analog detector is used to detect low and high concentration analytes in the same analysis.

\subsubsection{LA-ICP-MS Experimental Design}

\subsubsection{LA-ICP-MS Setup and Instrumentation}

A PerkinElmer Elan 6100 DRC (PerkinElmer, Waltham, MA, USA) ICP-MS was utilized for all LA-ICP-MS experiments presented in this chapter. The laser ablation unit utilized in the analysis of cotton have been previously described, section 4.3.3. Table 39 presents the laser ablation parameters and ICP-MS operating conditions optimized for this analysis.

The ICP utilized a $16 \mathrm{~L} / \mathrm{min}$ Ar plasma gas flow, $1 \mathrm{~L} / \mathrm{min}$ Ar auxillary gas flow and an entirely He ablation cell sweep gas. The He sweep gas ranged from $0.88-0.96 \mathrm{~L} / \mathrm{min}$. The sweep gas and ablated particles were merged using a "Y-type" connection (Thermo Scientific, Rochester, NY, USA) into a Ar nebulizer gas flow of 0.88-0.96 L/min. The range in sweep and nebulizer gas flows was used to optimize the conditions of the ICP plasma.

Optimal conditions involved monitoring the intensities of ${ }^{7} \mathrm{Li}^{139} \mathrm{La}$ and ${ }^{140} \mathrm{Ce}$ to ensure sensitivity spans the mass range. In addition, to minimize the presence of oxides and doubly charged species in the plasma the nebulizer and sweep gas flows were varied. Oxides and doubly charged were 
monitored by comparing $\mathrm{ThO} / \mathrm{Th}$ and/Ca++/Ca, respectively. The interferences could not account for more than $3 \%$ when compared to the analyte. These particular species were selected to monitor interferences because they are the most difficult oxides and doubly charged species to form in the ICP. If these species are $<3 \%$ all other oxides and doubly charged constituents will be negligible.

Table 39: LA-ICP-MS method for unprocessed cotton analysis

\begin{tabular}{|l|c|}
\hline \multicolumn{1}{|c|}{ Parameter } & Operating Condition \\
\hline Laser Ablation Unit & $2.91-2.96 \mathrm{~mJ}(80 \%)$ \\
\hline Laser energy output & $10 \mathrm{~Hz}$ \\
\hline Laser frequency $(\mathrm{Hz})$ & $\begin{array}{c}\text { Depth profile } \\
\text { (z rate }=0.25 \mathrm{~mm})\end{array}$ \\
\hline Ablation mode & 200 \\
\hline Spot size $(\mu \mathrm{m})$ & 40 \\
\hline Duration of ablation (seconds) & 16 \\
\hline ICP-MS unit & $0.88-0.96$ \\
\hline Plasma flow (L/min) & $0.88-0.96$ \\
\hline Nebulizer gas flow, & 1500 \\
\hline Ar (L/min) & 115 \\
\hline Sweep gas flow, He (L/min) & 2 \\
\hline Forward power $(\mathrm{W})$ & \\
\hline Readings/rep & \\
\hline Sweeps/reading &
\end{tabular}

Unlike LA-ICP-OES, LA-ICP-MS requires He as the sweep gas. The lower thermal conductivity of this gas plays a significant role in mitigating the formation of aggregates in the ICP. The MS is particularly sensitive to variation in particle size. The different modes of ICP detection, either by emission or isotopic means of inorganic MS were affected by the presence of helium. Since detection in LA-ICP-OES occurs directly in the plasma, lower flow rates and an environment that promotes energy transfer is necessary. However, LA-ICP-MS uses the ICP only as an ionization source from which the ions are quickly transferred to the interface region and finally the MS for separation and eventually, detection. The higher gas flow mixture (He-Ar) is needed to achieve a small uniform particle distribution. Lower, single gas flows lead to the formation of larger aggregates that do not have the opportunity for ionization in the fast moving central channel of 
the torch. This yields poor signal stability with erratic fluctuations. The use of He maintains a narrow particle size distribution resulting in a more consistent, thorough ionization in the plasma.

The mode of MS detection employed in this research is known as peak hopping. Peak hopping is used to save analysis time. In the MS voltages are applied to the ion lens which is used to direct specific species with a particular $m \backslash z$ value through the rods to the detector. In place of scanning the entire mass range of the MS, peak hopping quickly "jumps" the voltage to the specific value that corresponds to a particular $m \backslash z$ value. This quickens the ability to cycle through the analytes.

\subsubsection{Element Menu}

Several elements have been included in this research. Similarly to the categories of elements utilized for LA-ICP-OES and LIBS, the method elements of LA-ICP-MS have also been divided into detectable, monitored and element menu analytes. Elements selected to be included in the element menu on the basis of performance regarding the ability to see differences between cotton that originated from different geographic regions. Table 40 describes the different analytes detected and those then selected for the element menu.

Table 40: LA-ICP-MS element menu for unprocessed cotton analysis

\begin{tabular}{|c|c|}
\hline Analyte Category & Element isotope \\
\hline Detected & ${ }^{47} \mathrm{Ti},{ }^{51} \mathrm{~V},{ }^{66} \mathrm{Zn},{ }^{90} \mathrm{Zr}$ \\
\hline Monitored & ${ }^{42} \mathrm{Ca},{ }^{65} \mathrm{Cu},{ }^{23} \mathrm{Na}$ \\
\hline Element Menu & ${ }^{27} \mathrm{Al},{ }^{137} \mathrm{Ba},{ }^{57} \mathrm{Fe},{ }^{25} \mathrm{Mg},{ }^{55} \mathrm{Mn},{ }^{88} \mathrm{Sr},{ }^{13} \mathrm{C}$ \\
\hline
\end{tabular}

As a result of the sensitivity of LA-ICP-MS additional elements could be detected with this technique that could not be observed in atomic emission analysis. However, these analytes were not included in the element menu for several reasons. Some "detected" elements were typically present in the unprocessed cotton fiber at low concentration $(>10 \mathrm{ppm})$. The variation between samples was not significant to yield an improvement in the separation of the groups as 
determined by the geographic region. Also, these analytes were present at the low concentration were also not consistently detected with precision and followed no discernible trend regarding their presence. Other elements were considered redundant, as the concentration of these analytes (Zn, for example) varied similarly with other plant nutrients $(\mathrm{Mg})$.

\subsection{Results and Data Analysis}

\subsubsection{LA-ICP-MS Analytical Figures of Merit}

Precision and bias of the two standards, IAEA-V9 and AZ2006 are presented in tables 41 and 42, respectively. Precision and biases for LA-ICP-MS analysis of both the SRM and in-house standard range from good to excellent. These values were obtained over several analyses performed over approximately three months. The ability to achieve excellent bias is further demonstrated by the linearity of the calibration curves, figure 50, generated with LA-ICP-MS and the small standard deviations observed over broad concentration ranges.

Table 41: Analytical performance of LA-ICP-MS using IAEA V-9

\begin{tabular}{|c|c|c|c|}
\hline Element & Average conc., ppm & Precision (\% RSD) & Accuracy (\% bias) \\
\hline $\mathrm{Al}$ & 44 & 2.5 & - \\
\hline $\mathrm{Ba}$ & 9 & 1.7 & - \\
\hline $\mathrm{Fe}$ & 10. & 6.8 & - \\
\hline $\mathrm{Mg}$ & 53 & 1.9 & 12. \\
\hline $\mathrm{Mn}$ & 0.17 & 4.9 & -12. \\
\hline $\mathrm{Sr}$ & 0.57 & 1.1 & \\
\hline
\end{tabular}

Table 42: Analytical performance of LA-ICP-MS using AZ2006

\begin{tabular}{|c|c|c|c|}
\hline Element & Average conc., ppm & Precision (\% RSD) & Accuracy (\% bias) \\
\hline $\mathrm{Al}$ & 40. & 4.9 & 18 \\
\hline $\mathrm{Ba}$ & 1.1 & 5.4 & 22 \\
\hline $\mathrm{Fe}$ & 40. & 5.9 & 6.7 \\
\hline $\mathrm{Mg}$ & 600 & 2.0 & -1.0 \\
\hline $\mathrm{Mn}$ & 4.4 & 4.3 & -2.2 \\
\hline $\mathrm{Sr}$ & 5.1 & 5.9 & 13 \\
\hline
\end{tabular}


LA-ICP-MS is capable of performing excellent quantitative analysis whether the two standards are relatively close in concentration $(\mathrm{Ba})$, or significantly different $(\mathrm{Mg})$.

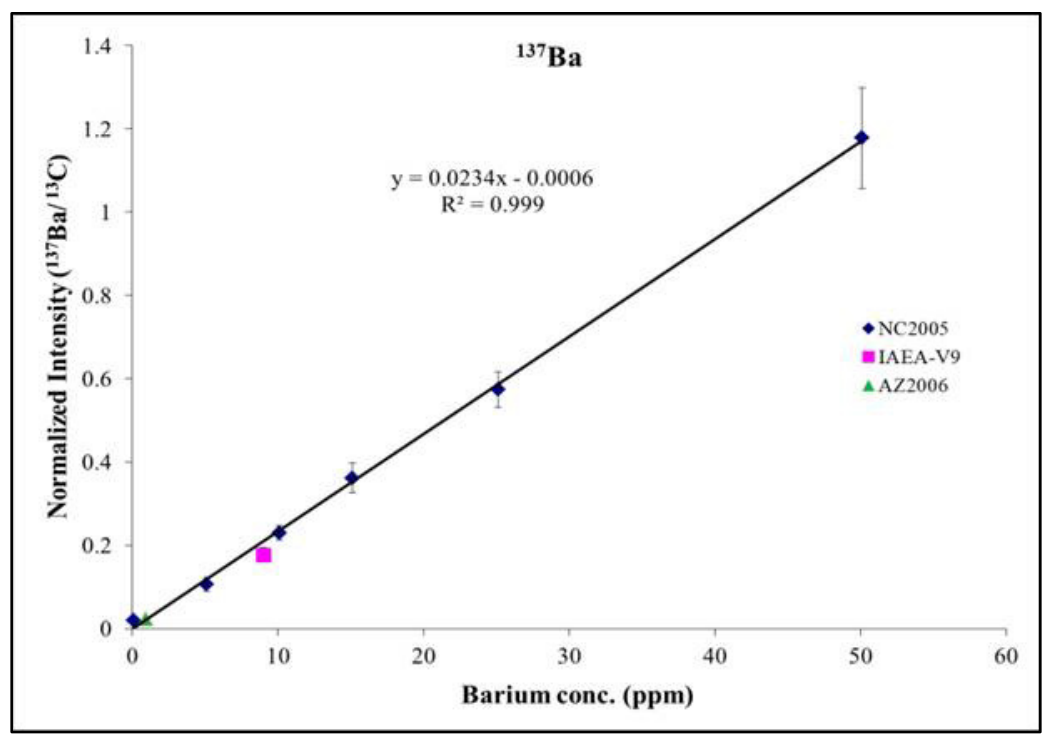

Figure 50a: LA-ICP-MS calibration curve using NC2005, example 1.

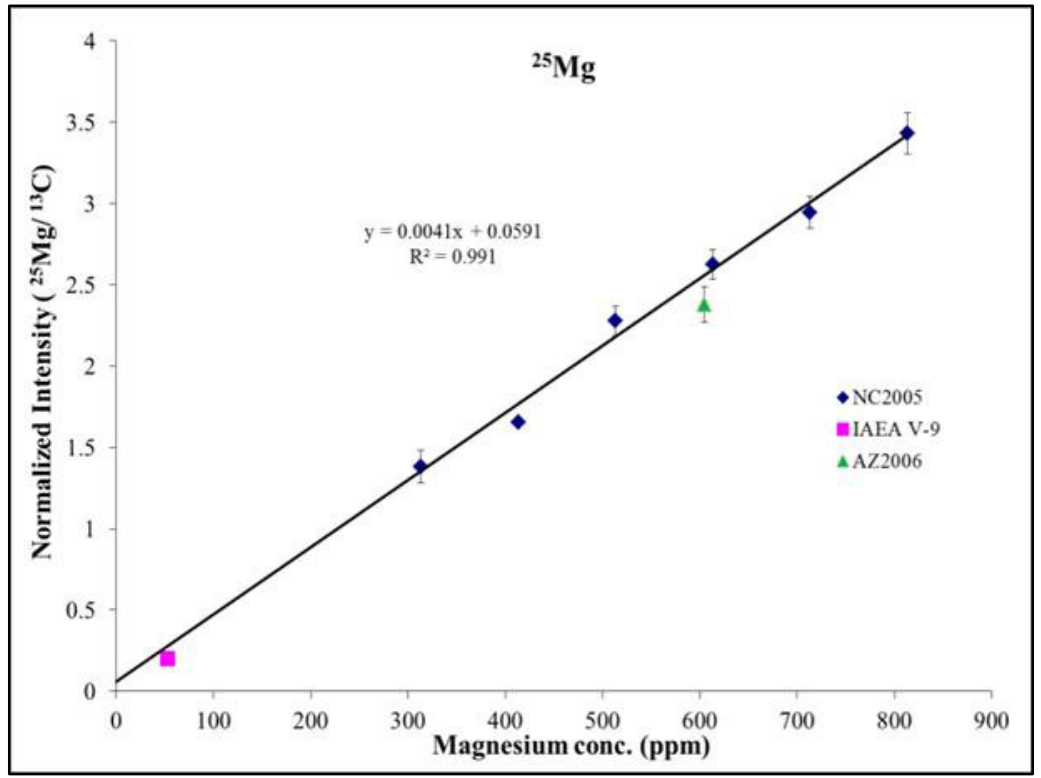

Figure 50b: LA-ICP-MS calibration curve using NC2005, example 2.

In addition, the achieved LODs, table 43, are presented. The LODs presented here have been determined using the approach outlined in equations 16 and 17 of this dissertation. The sensitivity 
of LA-ICP-MS is significantly beyond that which is needed to conduct the elemental analysis of unprocessed cotton. LA-ICP-MS is able to perform without the presence of non-detects.

Table 43: LA-ICP-MS LODs for unprocessed cotton analysis

\begin{tabular}{|c|c|}
\hline Element & LOD (ppm) \\
\hline $\mathrm{Al}$ & 0.090 \\
\hline $\mathrm{Ba}$ & 0.2 \\
\hline $\mathrm{Fe}$ & 0.11 \\
\hline $\mathrm{Mg}$ & 0.70 \\
\hline $\mathrm{Mn}$ & 0.10 \\
\hline $\mathrm{Sr}$ & 0.092 \\
\hline
\end{tabular}

The crater obtained using the laser ablation parameters for LA-ICP-MS analysis is presented in figure 51. Although the same laser ablation unit was used for both ICP techniques the optimized parameters were different. LA-ICP-MS utilized $80 \%$ laser output energy at a $10 \mathrm{~Hz}$ laser frequency, in comparison to the $90 \%$ and $5 \mathrm{~Hz}$ employed in LA-ICP-OES. LA-ICP-MS removes, $20.2 \mu \mathrm{g}$, twice as much mass as LA-ICP-OES analysis.

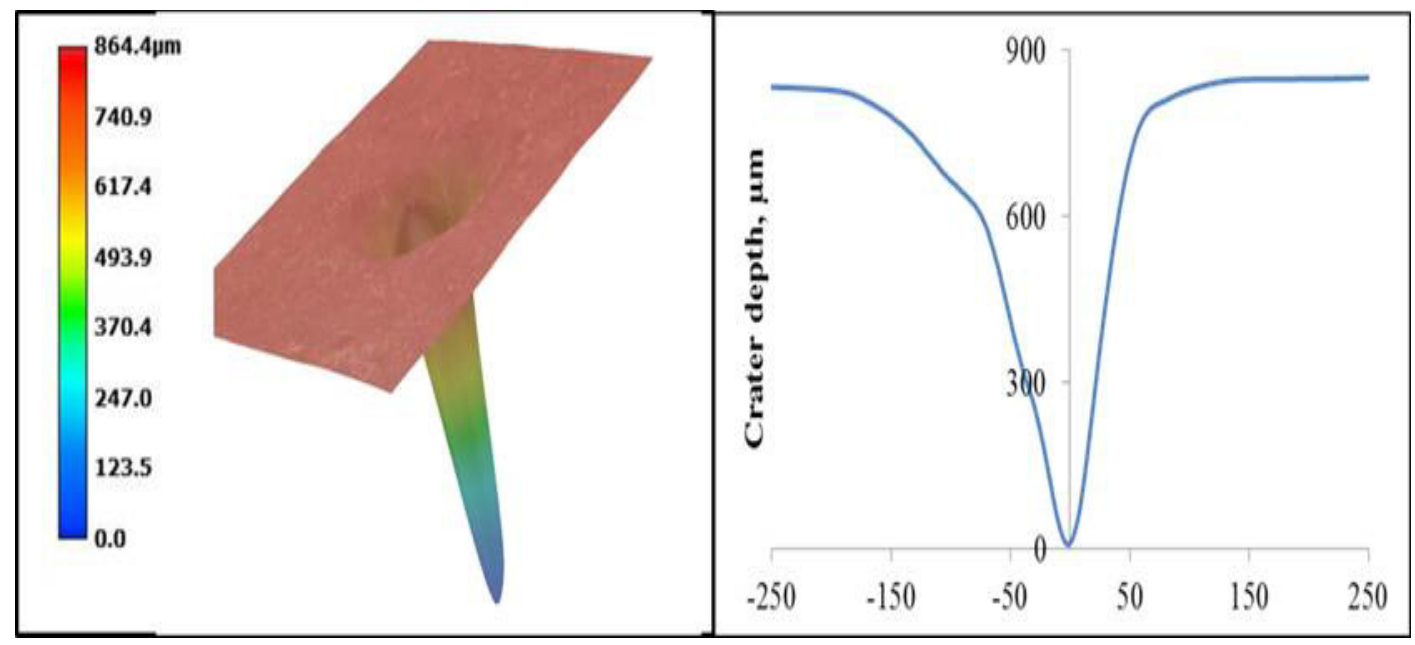

Figure 51: Typical LA-ICP-MS cotton crater; 3D image of cotton crater obtained using LA-ICP-MS (left). Side slice of crater at its deepest depth (right).

More mass is removed using a laser frequency twice that employed in LA-ICP-OES analysis. It should be noted that all elemental analysis techniques require the use of a pellet to perform the 
analysis so while there is a difference in mass required for each technique, this does not change or reduce the current sample preparation. There the difference is minimal.

\subsubsection{Performance Utilizing a Sample Set}

To determine the applicability of inorganic MS to the geographic provenancing of cotton and to make comparisons of the abilities of each elemental analysis technique using the same test sample set as utilized in LA-ICP-OES and LIBS analysis. Duplicate analysis in place of triplicate analysis was performed. Using the same element menu as in LA-ICP-OES and LIBS, LA-ICPMS is able to detect differences in elemental composition that pertain to differences in geographic region. Inorganic MS demonstrates comparable if not, better separation between the two geographic regions, figure 52. The LA-ICP-MS data for the two geographic regions are considered to be well separated since they fall into two different quadrants on the plot. Imagine a boundary drawn vertically at the zero point on PC 1; the two regions are almost completely separated by some distance using this boundary.

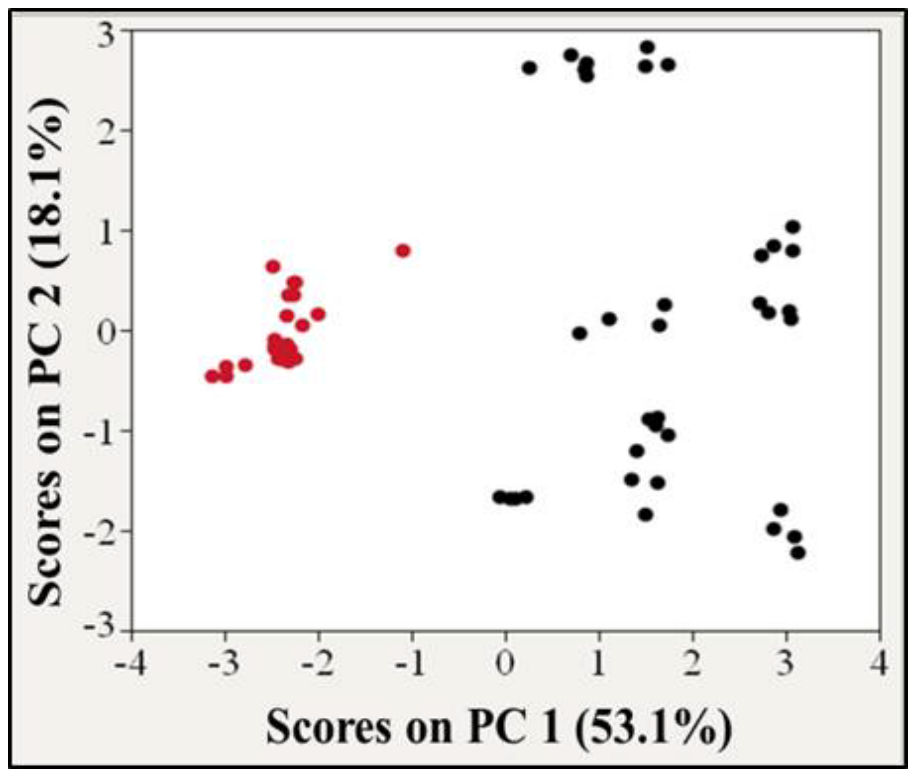

Figure 52: LA-ICP-MS PCA of cotton test sample set, black =southwest, red = southeast. 
However, the precision of LA-ICP-MS can also be somewhat of a disadvantage. The method appears to be sensitive to microheterogeneity in the samples and thus could explain the clustering of some replicates and even pellets encountered in the analysis. Microheterogeneity in the pellet can be exaggerated by excellent precision, leading to a misinterpretation of trends. For example, clustering of single pellet replicates is evident in figure 52 for southwest data. This too was observed to some extent in LA-ICP-OES score plots. This however, could also be exaggerated due to the number of samples analyzed. The sampling strategy presented earlier, section 6.3.1.1, developed for this research attempts to combat the potential microheterogeneity present. Although this may be problematic for small scale cotton analysis the focus of this research to develop a model to associate a sample to a group, i.e. geographic region; expanding the sample population to include temporal and spatially different samples aides in reducing this effect.

\subsubsection{Comparison to LA-ICP-OES and LIBS}

For the current application presented here, LA-ICP-MS is able to perform comparably, if not surpassing the analytical capabilities of both atomic emission techniques. However, although the ability to conduct measurements with such sensitivity is often desirable, the purpose of the application should be considered. While the sensitivity and precision of LA-ICP-MS may be coveted for distinguishing between samples, the ultimate goal here is class assignment. Correct class assignment relies on the 1) ability to detect analytes that contribute to group differences, 2) with the caveat that the technique can detect those analytes across a broad range of concentrations that can potentially be encountered and 3) can do so at a level of precision that exceeds the observed variation between the groups. All elemental techniques considered here could detect differences between regions with precision, i.e. NC2005 and AZ2006. Although all techniques evaluated in this dissertation were capable of achieving these three important qualifiers, the 
overall strengths and limitations of the aspects described in table 38 should also be considered when selecting the elemental analysis technique that is most suitable for this application.

The design and needs of the instrument are important for the current application as well as how amenable the technique is to incorporation into the laboratory for other uses. Table 44 presents an overall comparison of space needs as well as the extent of the laboratory budget that would be dedicated to instrument installation and maintenance should be contemplated in combination with the analytical performance of the techniques. While the cost and requirements of a commercial LIBS system is less than that required for either ICP technique prior to the coupling of laser ablation, the difference in performance and skill set required might offset the cost.

In addition, the less time required for maintenance, as in ICP-OES and LIBS, decreases instrument down-time and results in more time to dedicate to the improvement of skill set of the analyst to overcome difficulty in data output and interpretation. 
Table 44: Comparisons of elemental analysis techniques

\begin{tabular}{|c|c|c|c|}
\hline Property & LA-ICP-MS & LA-ICP-OES & LIBS \\
\hline \multicolumn{4}{|c|}{ Instrumental Design } \\
\hline Capital cost & $\begin{array}{l}\text { Laser }=\$ 80-100 \mathrm{~K} \\
\mathrm{ICP}=\$ 150 \mathrm{~K}\end{array}$ & $\begin{array}{l}\text { Laser }=\$ 80-100 \mathrm{~K} \\
\mathrm{ICP}=\$ 80-120 \mathrm{~K}\end{array}$ & $\begin{array}{l}\text { In-house }=\$ 50-100 \mathrm{~K} \\
\text { Commercial }=\$ 70-120 \mathrm{~K}\end{array}$ \\
\hline $\begin{array}{l}\text { Instrumental } \\
\text { requirements }\end{array}$ & $\begin{array}{l}\text { Ar \& He gas; } \\
\text { chiller; exhaust } \\
\text { system; vacuum } \\
\text { pumps }\end{array}$ & $\begin{array}{l}\text { Ar \& Ni gas; } \\
\text { chiller \& outdoor } \\
\text { compressor; exhaust } \\
\text { system }\end{array}$ & Ar gas \\
\hline $\begin{array}{l}\text { Instrument } \\
\text { flexible }\end{array}$ & $\begin{array}{l}\text { No unattended } \\
\text { operation, multi- } \\
\text { element technique }\end{array}$ & $\begin{array}{l}\text { No unattended } \\
\text { operation, multi- } \\
\text { element technique }\end{array}$ & $\begin{array}{l}\text { No unattended operation, } \\
\text { multi-element technique }\end{array}$ \\
\hline \multicolumn{4}{|c|}{ Ease of use (skill level required) } \\
\hline $\begin{array}{l}\begin{array}{l}\text { Performing } \\
\text { analysis }\end{array} \\
\end{array}$ & Moderate & Moderate & $\begin{array}{l}\text { In-house }=\text { expert } \\
\text { Commercial }=\text { moderate }\end{array}$ \\
\hline $\begin{array}{l}\text { Report } \\
\text { generation }\end{array}$ & Easy & Difficult & $\begin{array}{l}\text { In-house }=\text { difficult } \\
\text { Commercial = moderate }\end{array}$ \\
\hline Maintenance & Difficult & Easy & Easy \\
\hline \multicolumn{4}{|c|}{ Analytical Performance (for cotton) } \\
\hline $\begin{array}{l}\begin{array}{l}\text { Short-term } \\
\text { stability }\end{array} \\
\end{array}$ & Excellent & Excellent & Excellent \\
\hline $\begin{array}{l}\text { Long-term } \\
\text { stability }\end{array}$ & Excellent & Good to excellent & Good \\
\hline Sensitivity & Excellent & Good to excellent & Good to excellent \\
\hline $\begin{array}{l}\text { Dynamic } \\
\text { range }\end{array}$ & $\begin{array}{l}\text { Good for intended } \\
\text { use }\end{array}$ & $\begin{array}{l}\text { Good for intended } \\
\text { use }\end{array}$ & Good for intended use \\
\hline \multicolumn{4}{|l|}{ Sample related } \\
\hline Preparation & Pellets & Pellets & Pellets \\
\hline Sample size & $\sim 20 \mu \mathrm{g} /$ replicate & $\sim 10 \mu \mathrm{g} /$ replicate & $\sim 30 \mu \mathrm{g} /$ replicate \\
\hline
\end{tabular}

Cells colored in red imply that, for the particularly category, that technique surpasses the qualities of the other analytical tools presented.

The selection of the most suitable technique may vary from laboratory to laboratory, however the current research, in addition to accomplishing geographic provenancing of the matrix, also attempts to provide an unbiased approach of scrutinizing the abilities of the three techniques.

\subsection{Discussion and Conclusions}

Elemental analysis, regardless of the laser-based technique used, is capable of identifying differences in unprocessed cotton that can be attributed to different geographic regions. However, although all approaches could accomplish this using a small scale sample set, it is important to 
select the most appropriate analytical tool for performing this analysis in the long-term. While the ultimate decision cannot be made without additional analyses to result in the modeling of the data, which would in turn address the stability in the forecasting of the techniques, a general indication of the aptness of each approach can be made under the current circumstances.

As demonstrated by the results here, LA-ICP-MS offers excellent performance for this application, validating the use of the term "gold standard" which is often used to describe this analytical tool. However, the necessity of this caliber of performance is not warranted for this application and the cost of maintenance may reduce the attraction to this instrument. Although LA-ICP-OES does not differ significantly in the cost to acquire in comparison to LA-ICP-MS, other aspects - simplicity in maintenance particularly, is an attractive feature for the end user. In several instances forensic laboratories often have a back log of samples to be analyzed. Less time consuming maintenance results in less down time on the instrument and can alleviate back logs for this application and others as well.

It should also be addressed that the coupling of laser ablation to ICP-OES for sample analysis is relatively new. The majority of LA-ICP-OES analyses have been performed in the interest of research and therefore data exporting and tools to aide in the interpretation are not as advanced as in LA-ICP-MS. However, the same could have been said in the initial commercialization of LIBS, just a few years previous. The interest in the technique aides in the drive for advancement, as can be demonstrated in the significant improvements in commercial LIBS system that have been experienced first-hand in our laboratory.

On the basis of the current assessments of the three analytical tools, both LA-ICP-OES and LIBS appear to be suitable candidates for this application. However, all additional unprocessed cotton samples have been analyzed by each technique in order to reach the ultimate decision. 


\subsection{DETERMINATION OF THE STABLE ISOTOPES IN UNPROCESSED COTTON}

\subsection{Overview}

Previous chapters have demonstrated that the elemental profile of unprocessed cotton can be used to make associations to the region from which the plant originated. The measurement of stable isotope ratios $(\delta$, in \%o $)$ has also been shown in the literature to serve as an additional chemical signature that is characteristic of a geographic region. In plants, variations in the abundances of carbon $\left(\delta^{13} \mathrm{C}\right)$, oxygen $\left(\delta^{18} \mathrm{O}\right)$ and hydrogen $\left(\delta^{2} \mathrm{H}\right.$ or $\left.\delta \mathrm{D}\right)$ isotopes can be associated to differences in growing regions and climate conditions. These differences vary by region and it is hypothesized, will vary similarly in the degree in which the isotope ratios manifest in the cotton boll. The current chapter presents the analysis of unprocessed cotton samples of both domestic and foreign origin to determine the extent of variation in stable isotope ratios that is related to geographic regions. Studies are conducted to assess the temporal and spatial variation in stable isotope ratios using unprocessed domestic cotton. Because of the significantly smaller sample population available for foreign cotton analysis domestic cotton is utilized as a means to measure the proof of concept, which is then applied to the foreign samples.

\subsection{Introduction}

\subsubsection{Stable Isotopes}

All elements of the periodic table exist in some form, as an isotope. Elements may be monoisotopic (single isotope) or have several isotopes that vary in abundance. The term "isotope" was coined by Frederick Soddy ${ }^{144}$. Simply put, "isotope" means "in equal position ${ }^{144,}$. As previously described, an atom is composed on a nucleus made up of protons and neutrons, 
surrounded by an electron cloud. An isotope of a given element varies in the number of neutrons, but has the same number or protons and electrons. Because the number of neutrons does not affect the number of protons or electrons, each isotope of a particular element has similar chemical properties. However, although the chemical properties may be the same, mass dependent properties vary with each isotope.

The abundance of the isotopes for each element is fixed in nature. However, when considering the light or stable isotopes - carbon, hydrogen, oxygen, nitrogen and sulfur - while the overall abundance may be fixed, isotopes of these elements are subject to fractionation. Fractionation is the process in which the amount of heavy and light isotopes fluctuates as a result of various biological, biochemical, chemical and physical processes that are affected by mass differences ${ }^{155}$. These processes are considered isotopic effects ${ }^{155}$.

Isotope fractionation can occur in one of two, or a combination of both ways. Fractionation can result from kinetic isotopic effects or thermodynamic isotopic effects. Kinetic effects refer to any mass discrimination that is the result of chemical reactions. For example, bonds that incorporate heavier isotopes break at a much slower rate than bonds between lighter isotopes ${ }^{155}$. The rate of a chemical reaction then is determined by the presence of the different isotopes.

The second type of fractionation does not deal with the breaking or forming of bonds.

Thermodynamic isotopic effects can often be thought of equilibrium effects. Of particular interest to this research is the fractionation that initially results from condensation, evaporation and diffusion, however, these are not the only thermodynamic isotopic effects. Lighter isotopes more readily evaporate while heavier isotopes condense more easily. This results in further depletion or enrichment of the isotopes during different kinetic effects described above.

\subsubsection{Use of Stable Isotopes for Geographic Provenancing}


Variations in stable isotope ratios can be used to make associations to a particular geographic location or region. This has been demonstrated in the literature with illicit drugs ${ }^{156-158}$, wine ${ }^{159}$ and other forensic related applications ${ }^{160}$. In addition a small proof of concept study using seven samples of unprocessed cotton fibers was able to be differentiated on the basis of country of $\operatorname{origin}^{161}$. The ability to make these associations using stable isotope ratios is initially the result of variations in climate from region to region. The isotopes are incorporated into the material through processes that result in further fractionation by thermodynamic and kinetic means.

Oxygen and hydrogen isotope ratios vary on the basis of precipitation, condensation and evaporation of water and therefore contain regional climate information. These processes are all thermodynamic isotopic effects. All water sources in the earth are grouped together as one of earth's spheres, the hydrosphere. Water sources are not limited to just bodies of water, but also include ground and soil water as well. Water within this sphere is in a constant state of movement in different physical states and across earth's spheres; this movement is known as the hydrological cycle ${ }^{155}$. The movement depends on geographical factors such as distance from a body of water, temperature, altitude and latitude ${ }^{155}$. Variations are spatial, but can also change with season, to produce temporal variation in $\delta^{2} \mathrm{H}$ and $\delta^{18} \mathrm{O}$ as well. The changes in climate from region to region result in different degrees of enrichment, or depletion of a particular isotope. For example, as temperature increases and water evaporates, the light isotopes will more readily evaporate. As a result there is an enrichment in the lighter isotopes in water vapor. This enrichment extends upwards as the vapor begins to cool; the resulting precipitation is enriched in the heavier isotopes. In addition to these enrichment/depletion trends that revolve around climate conditions there are additional factors regarding the movement of air masses. The air masses move the vapors (clouds) further from bodies of water ${ }^{155}$. A general correlation of these trends is depicted in figure 53. Stable isotope information, like that which is presented in figure 53, utilizes 
delta $(\delta)$ notation to depict the enrichment and depletion of the heavy and light isotope for a particular element in comparison to a standard.

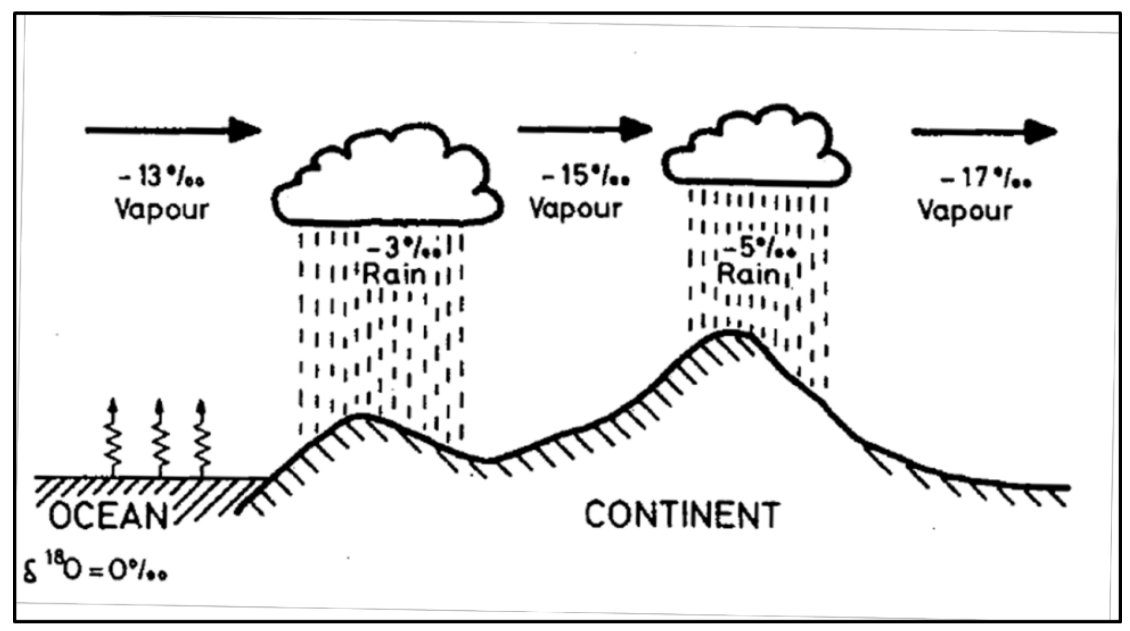

Figure 53: $\delta^{18} \mathrm{O}$ Stable isotopes in nature ${ }^{155}$.

The relationship between the sample isotope ratio $\left(\mathrm{R}_{\text {sample }}\right)$ and the stable isotope ratio of the standard $\left(\mathrm{R}_{\text {std }}\right)$ can be found in equation 19.

$$
\delta_{\text {sample }}=\left(\frac{\left[R_{\text {sample }}-R_{\text {std }}\right\rfloor}{R_{\text {std }}}\right) \times 1000
$$

Specific standards are used as points of reference. The standards include the Vienna Standard Mean Ocean Water (VSMOW) for hydrogen and oxygen analysis and the Vienna Pee Dee Belemnite (VPDB) standard for carbon analysis. A positive $\delta$ value for the sample means that it contains more of the heavy isotope, while a negative $\delta$ values corresponds to a sample that contains less of the heavy isotope. The values of the sample are in terms of the standard used in equation 19. Because these differences occur on such a small scale, they are represented in "per mil" notation (\%o) instead of percent. 
The differences in both hydrogen and oxygen isotope ratios are related by the $\delta \mathrm{D}-\delta^{18} \mathrm{O}$ relationship ${ }^{162-164}$. When considering isotopic effects that are correlated with climate conditions evaporation, precipitation or condensation - the hydrogen and oxygen isotope fractionation is proportional. The general association is depicted by the "Global Meteoric Water Line ${ }^{163,163,164}$." This association is constantly evaluated by additional comparisons made at data collection stations located throughout the world ${ }^{165}$. This information is compiled into monthly and yearly maps that illustrate isotopic variations, typically using a color scale. These maps are referred to as isoscapes $^{165}$.

Plants, like cotton, take up water through their root system and use this water in different biochemical and physicochemical processes. There is no fractionation during water uptake from the soil ${ }^{166,167}$, however different components of the plant are subject to additional thermodynamic effects, i.e. transpiration (evaporation), and have demonstrated different $\delta^{18} \mathrm{O}$ ratios ${ }^{168,169}$. Other fractionation during chemical or biochemical reactions result from kinetic isotopic effects. For example, the incorporation of oxygen from water into cellulose and other carbohydrates, proteins and plant lignins results in different contributions to fraction ${ }^{155}$. The resulting $\delta^{2} \mathrm{H}$ and $\delta^{18} \mathrm{O}$ isotope ratio of the cotton boll are expected to be comprised of plant processes information (both thermodynamic and kinetic) and regional climate information (mainly thermodynamic). The processes within the plant are indirectly related to the region, in that the processes require organic constituents, i.e. carbon, hydrogen and oxygen in order to function, and thus are also expected to provide spatial information regarding geographic origin. This has been demonstrated in the literature, accomplishing the geographic provenancing of marijuana plants ${ }^{158}$ as well as tracing geographic travel by means of human hair analysis ${ }^{170}$.

The $\delta^{13} \mathrm{C}$ varies for different types of plants. Plants vary in the manner in which they take up extracellular or atmospheric $\mathrm{CO}_{2}$ and then incorporate this into plant material ${ }^{155}$; this is known as 
photosynthesis. The first step, extracellular to intracellular $\mathrm{CO}_{2}$, is accomplished by diffusion and is reversible; however the fixation of carbon is irreversible ${ }^{155}$. The differences in pathways that can be used in the biological fixation of carbon are determined by plant type. Cotton is a $\mathrm{C}_{3}$ plant, so carbon fixation and $\delta^{13} \mathrm{C}$ values will only be described in terms of this pathway. All $\mathrm{C}_{3}$ plants use the Calvin cycle. The $\mathrm{C}_{3}$ plants use ribulose bisphophate carboxylase oxygenase (rubisco) to react with $\mathrm{CO}_{2}$ to yield 3-phosphoglyceric acid ${ }^{155}$. From this the carbon is reduced and carbohydrates (sugars) are produced; ribulose bisphophate is also formed ${ }^{155}$. The change in $\delta^{13} \mathrm{C}$ is the result of kinetic isotopic effects.

The sugar that is produced can then be used in subsequent processes throughout the cell to generate other important compounds, cellulose etc. The $\delta^{13} \mathrm{C}$ for the different photosynthesis pathways in plants (focusing on $\mathrm{C}_{3}$ and $\mathrm{C}_{4}$ ) do not overlap, suggesting that the prominent contributions to ${ }^{13} \mathrm{C}$ content is due to differences in metabolic pathways, and thus plant type can be distinguished using this information ${ }^{155}$. To differentiate from within a plant type, $\mathrm{C}_{3}$ plants in this research, environmental factors that contribute to the atmospheric $\mathrm{CO}_{2}$ uptake rate, for example temperature and altitude - are important ${ }^{156}$. The differences are not as significant as in $\delta^{2} \mathrm{H}$ and $\delta^{18} \mathrm{O}$ climate variations, and so it expected that the differences in $\delta^{13} \mathrm{C}$ between cotton samples may not depict the true variation between the geographic regions. 


\subsection{Materials and Methods}

\subsubsection{Description of Samples and Sample Preparation}

\subsubsection{Description of Samples}

All samples submitted for IRMS analysis were in powder form. The powder was split from the same milled portions of cotton that were used to generate the pellets for elemental analysis. Because all samples were split from the same bulk batch a direct comparison between stable isotope ratio and elemental profile can be made.

\subsection{Domestic Unprocessed Cotton}

The samples for this study were selected to explore temporal and spatial variations of the stable isotopes of unprocessed cotton. The purpose of this research was to assess the applicability of stable isotope results to the geographic provenancing of unprocessed cotton by providing a proof of concept. A small set of samples was selected for this analysis in part because this study was outsourced. Helen Kemp and Professor Wolfram Meier-Augenstein graciously provided the services of the MRS stable isotope facility for this research.

To evaluate spatial variation within the U.S., one sample from several different states were selected spanning the cotton belt. All samples were collected from the 2009 harvest year. The samples were used to determine variation on a large scale, i.e. the southern U.S

To further evaluate spatial heterogeneity five additional samples were selected from five different counties in North Carolina. All samples were from the 2009 harvest as well. The North Carolina county samples and the other 2009 crops were selected to determine the extent to which regional variation could be detected. 
Finally, to provide some perspective on temporal variation, two additional samples from North Carolina were selected for analysis. The samples were from the 2005 and 2006 harvests. Table 45 provides a complete description of the domestic samples analyzed by IRMS.

Table 45: Domestic cotton sample information for IRMS analysis

\begin{tabular}{|c|c|c|}
\hline Sample No. & Location & Harvest year \\
\hline 1 & Florence, South Carolina & 2009 \\
\hline 2 & Las Crucas, New Mexico & 2009 \\
\hline 3 & Lubbock, Texas & 2009 \\
\hline 4 & Jay, Florida & 2009 \\
\hline 5 & Yorkville, Tennessee & 2009 \\
\hline 6 & Nashville, Georgia & 2009 \\
\hline 7 & Leachville, Arkansas & 2009 \\
\hline 8 & Theodore, Alabama & 2009 \\
\hline 9 & Glendora, Mississippi & 2009 \\
\hline 10 & Whitacker, North Carolina & 2009 \\
\hline 11 & Dunn, North Carolina & 2009 \\
\hline 12 & Fairfield, North Carolina & 2009 \\
\hline 13 & Garysburg, North Carolina & 2009 \\
\hline 14 & Elm City, North Carolina & 2009 \\
\hline 15 & Ayden, North Carolina & 2009 \\
\hline 16 & North Carolina & 2005 \\
\hline 17 & North Carolina & 2006 \\
\hline
\end{tabular}

The table has been color coded to distinguish between the purpose for sample inclusion.

\subsection{Foreign Unprocessed Cotton}

All foreign unprocessed cotton samples analyzed by elemental analysis were also analyzed by IRMS. A subset of samples was not selected as in the domestic samples mostly because of the much smaller sample pool of foreign unprocessed cotton available.

Although on a small scale, the purpose of the analyses of the foreign cotton samples were to serve as a proof of concept that there are differences on the basis of stable isotope ratios between different regions or countries of the world. Particularly, countries or regions involved in the exportation and importation of this commodity. Table 46 contains the sample information for the foreign samples analyzed. 
Table 46: Foreign cotton sample information

\begin{tabular}{|c|c|}
\hline Sample Number & Location \\
\hline 1 & China \\
\hline 2 & China \\
\hline 3 & Mexicali, Mexico \\
\hline 4 & Chihuahua, Mexico \\
\hline 5 & Chihuahua, Mexico \\
\hline 6 & Mexicali, Mexico \\
\hline 7 & Pakistan \\
\hline 8 & Pakistan \\
\hline 9 & Tanzania \\
\hline 10 & Tanzania \\
\hline 11 & India \\
\hline 12 & India \\
\hline 13 & Brazil \\
\hline 14 & Brazil \\
\hline 15 & China \\
\hline 16 & China \\
\hline 17 & China \\
\hline 18 & China \\
\hline 19 & Senegal \\
\hline 20 & China \\
\hline 21 & Zimbabwe \\
\hline 22 & Guadalupe \\
\hline
\end{tabular}

\subsubsection{Sample Preparation}

Sample preparation was conducted by the MRS stable isotope facility. Small amount of sample $(\sim 10 \mathrm{mg})$ was needed in order to accomplish both the ${ }^{2} \mathrm{H}$ and ${ }^{18} \mathrm{O}$ and the ${ }^{13} \mathrm{C}$ analyses in triplicate. The samples are dried in a desiccator prior to sampling. The glass desiccator was sealed with high vacuum grease to avoid any leaks. Silver capsules were used for ${ }^{2} \mathrm{H}$ and ${ }^{18} \mathrm{O}$ analysis, tin capsules for ${ }^{13} \mathrm{C}$ analysis. The sample capsules were crimped into balls.

\subsection{Equilibration of Samples}

An equilibration procedure was used for the samples being analyzed for ${ }^{2} \mathrm{H}$ and ${ }^{18} \mathrm{O}^{170}$. Equilibration of the samples is conducted in order to extract the true $\delta^{2} \mathrm{H}$ from the total $\delta^{2} \mathrm{H}$ amount. The true $\delta^{2} \mathrm{H}$ value is considered to be the "non-exchangeable" content, referring to that which cannot act as $\mathrm{H}^{+}$acid ${ }^{144}$. 
Subsets are split and stored in one of two desiccators. Each desiccator contains a water sample of known isotope ratio. The water samples should differ by $100 \%$. The subsets are left to equilibrate for 4 days. Small sets ( 7 samples) were used to avoid hydrogen-crossover ${ }^{170}$. The samples were then transferred and dried down under vacuum for seven days. Allowing the samples to equilibrate in the same conditions as a known isotope ratio water sample allows for the determination of the exchangeable hydrogen amount. This allows the sample to equilibrate in water vapor of known isotopic concentration ${ }^{170}$.This results in the complete equilibrium of exchangeable hydrogen in the sample with the known water vapor. The $\delta^{2} \mathrm{H}$ isotope ratio can then be determined to be that which is only attributed to the non-exchangeable hydrogen content. The exchangeable and nonexchangeable are thought of as fractions of the whole. This concept is used to explain the ability to separate these two constituents.

\subsubsection{Instrumentation}

\subsubsection{Principles of IRMS}

The instrumental setup utilized for isotope ratio mass spectrometry (IRMS) differs from a typical mass spectrometer, like that which is used in the ICP technique. Differences in the abundances of the heavy and light isotopes are usually very small; such a value is beyond the accuracy limits of a typical mass spectrometer. Mass spectrometers capable of conducting stable isotope analysis are focused on accuracy and sensitivity capabilities, not as much resolution which is significant in normal analysis utilizing a mass spectrometer.

There are several IRMS instruments available, however this research utilized a continuous flow IRMS (CF-IRMS) for bulk stable isotope analysis so only a description of that particular instrument configuration will be presented. A temperature conversion elemental analyzer IRMS 
(TC/EA IRMS) was the particular CF instrument used for ${ }^{2} \mathrm{H}$ and ${ }^{18} \mathrm{O}$ analysis. A modern combustion/reduction elemental analysis (EA-IRMS) was used for ${ }^{13} \mathrm{C}$ analysis.

Bulk stable isotope analysis of solid samples employs small silver (for ${ }^{2} \mathrm{H}$ and ${ }^{18} \mathrm{O}$ analysis) or tin capsules $\left({ }^{13} \mathrm{C}\right.$ analysis $)$ of the material in powder form in the presence of an oxidant. The sample undergoes complete combustion to produce the following gases: $\mathrm{N}_{2}, \mathrm{NO}_{\mathrm{x}}, \mathrm{CO}_{2}$ and $\mathrm{H}_{2} \mathrm{O}$ in a $\mathrm{He}$ environment with an excess of oxygen ${ }^{144}$. Helium not only acts as the environment, but also serves as a carrier gas to transport the products of the sample. The sample in gas form is then transported to a reduction furnace which is used to remove the oxygen and also converts the $\mathrm{NO}_{\mathrm{x}}$ to $\mathrm{N}_{2}$.

Both analysis of ${ }^{2} \mathrm{H}$ and ${ }^{18} \mathrm{O}$, and ${ }^{15} \mathrm{~N}$ and ${ }^{13} \mathrm{C}$ can be analyzed in a single run on the particular instruments listed above. Both of these instances are examples of dual-isotope analysis ${ }^{144}$.The analysis of ${ }^{15} \mathrm{~N}$ and ${ }^{13} \mathrm{C}$ at the same time can only be accomplished if the chromatography peaks corresponding to $\mathrm{N}_{2}$ and $\mathrm{CO}$ are sufficiently separated. On the other hand, chemical reactions are required prior to separation and detection for ${ }^{2} \mathrm{H}$ and ${ }^{18} \mathrm{O}$ analysis. Oxygen containing samples utilize a pyrolytic reaction for complete conversion to $\mathrm{CO}$. The carbon, at high temperatures $>$ $1400^{\circ} \mathrm{C}$ is also used to convert the sample and any remaining water to $\mathrm{H}_{2}$. The source of carbon is known as "glassy carbon ${ }^{144}$."

Separation is accomplished using a GC column. Following separation the sample is introduced into the inlet of IRMS. A flow of the reference gas is directed straight into the IRMS. The sample peaks are bracketed by the UHP reference gas.

An IRMS is different from a typical MS system. It lacks the flexibility in scanning a broad mass range, as it requires a dedicated detector for each $\mathrm{m} / \mathrm{z}$ ratio being analyzed. Typically an instrument is equipped with two or three detectors. Faraday cups are used as the detector source 
in an IRMS instrument. The cup is grounded. As the ion current passes through the ground the drop in resistance is measured and equated to the abundance of the particular $\mathrm{m} / \mathrm{z}$ ratio. The ions have to be directed to the particular faraday cups. This is accomplished using a magnet which deflects the different $\mathrm{m} / \mathrm{z}$ ratios at different angles. Only specific $\mathrm{m} / \mathrm{z}$ ratios reach the designated faraday cup. Faraday cups are extremely rugged and have good long-term stability. The design of the instrument explains why resolution is not a significant factor in this type of mass spectrometer.

\subsubsection{IRMS Experimental Design}

The methods and experimental design have been obtained from the MRS stable isotope facility in Dundee, Scotland.

\subsubsection{IRMS Setup and Instrumentation}

\subsection{IRMS for $\delta^{13} \mathrm{C}$ Analysis}

The samples, following the dry down stage, were transported to a Costech Zero-Blank autosampler (Pelican Scientific Ltd, Alford, United Kingdom) that was purged with He. Samples were analyzed using an automated nitrogen-carbon elemental analyzer (ANCA, SerCon Ltd, Crewe, UK) coupled to an IRMS (SerCon, Crewe, UK). The glass tubes used in the pyrolysis state contained chromium (III) oxide/copper oxide and reduced copper. Combustion was conducted at $1020^{\circ} \mathrm{C}$ and reduction at $620^{\circ} \mathrm{C}$. Water traps were placed in-line with the glass tubes so as to remove any water generated as a product of combustion. A GC column, held at a stable temperature of $65^{\circ} \mathrm{C}$ was used to separate $\mathrm{N}_{2}$ and $\mathrm{CO}_{2}$. All reference gases were ultra-pure using international methods. 


\subsection{IRMS for $\delta^{2} \mathrm{H}$ and $\delta^{18} \mathrm{O}$ Analysis}

The samples, following the equilibration process, were transported to a Costech Zero-Blank autosampler (Pelican Scientific Ltd, Alford, United Kingdom) that was purged with He.

A high temperature conversion-elemental analyzer was coupled to a Delta ${ }^{\text {Plus }}$-XP IRMS (Thermo Scientific, Brendon, Germany). The inside of the reactor filled with glass carbon granules. The tube was frequently repacked. The reactor temperature was kept at $1425^{\circ} \mathrm{C}$. The $\mathrm{GC}$ column was kept at $85^{\circ} \mathrm{C}$. A He gas $(99.999 \%$ pure $)$ flow of $90 \mathrm{~mL} / \mathrm{min}(1.45 \mathrm{bar})$ was used as the carrier gas in the instrument. Reference gases used were also ultra-pure and calibrated using international methods.

\subsection{Results and Data Analysis}

All unprocessed cotton samples and analytical QC samples have been scaled to account for variation in the ratio obtained for the standard used in the respective technique. The use of scaling has been thoroughly documented in the literature ${ }^{171,172}$.

\subsubsection{Analytical Performance}

Several standards are used in order to assure the accuracy and precision of both the instrumentation and the results obtained with the laboratory procedures. A USGS-40 SRM, Lglutamic acid, of known and certified isotopic composition is analyzed throughout each analytical run at the beginning, middle and end, ensuring the comparability of samples run at different times in the same analysis, much similar to what has been done in elemental analysis. The $\delta^{13} \mathrm{C}$ analysis could be conducted in two analytical runs, one of the domestic samples and of the foreign. To demonstrate the short and long-term stability of the technique table 47 presents the results 
obtained for USGS-40. The accepted value for $\delta^{13} \mathrm{C}$ in this SRM is $-26.39 \%$. The intra and interrun variability are good for the standard.

Table 47: Analytical performance of USGS-40 for $\delta^{13} \mathrm{C}$ analysis

\begin{tabular}{|c|l|c|c|}
\hline Run no. & Time analyzed & Average ( $\boldsymbol{\delta}$, per mil) & Std. deviation \\
\hline \multirow{3}{*}{1} & Beginning & -26.36 & 0.04 \\
\cline { 2 - 4 } & Middle & -26.40 & 0.03 \\
\cline { 2 - 4 } & End & -26.47 & 0.04 \\
\hline \multirow{3}{*}{2} & Beginning & -26.36 & 0.17 \\
\cline { 2 - 4 } & Middle & -26.31 & 0.06 \\
\cline { 2 - 4 } & End & -26.37 & 0.08 \\
\hline \multicolumn{2}{|c|}{ Long-term stability } & $\mathbf{- 2 6 . 3 8}$ & $\mathbf{0 . 0 5}$ \\
\hline
\end{tabular}

In addition two analytical QC samples of different concentration were analyzed, another glutamic acid and a nicotinamide standard. A secondary SRM was also analyzed in the middle of each analytical run. The SRM, IAEA-CH-6, sucrose, has a much different $\delta^{13} \mathrm{C}$ content $(-10.45 \%$ ), which allows for the ability to predict ${ }^{13} \mathrm{C} /{ }^{12} \mathrm{C}$ content that differs from the primary standard.

Table 48: Analytical performance of IAEA-CH-6 for $\delta^{13} \mathrm{C}$ analysis

\begin{tabular}{|c|c|c|}
\hline Run no. & Average ( $\boldsymbol{\delta}$, per mil) & Std. deviation \\
\hline 1 & -10.45 & 0.13 \\
\hline 2 & -10.45 & 0.17 \\
\hline
\end{tabular}

Two SRMs of differing oxygen isotopic composition were used in the analysis of the unprocessed cotton samples, table 49. Following inclusion into cellulose and other plant material, oxygen enrichment for $\mathrm{C}_{3}$ plants is approximately $27 \%{ }^{167,173}$. Variations that occur are the result of variations in the source water composition ${ }^{155}$. Because of the enrichment, in comparison to the SRM, the two selected differ extensively and bracket the variation expected in the samples. The SRMs, IAEA-601 and IAEA-602, are both benzoic acid standards with $\delta^{18} \mathrm{O}$ values of $23.30 \%$ and $71.40 \%$, respectively. Performance does deteriorate somewhat when the sample is significantly enriched with the heavier isotope in comparison to the standard, as can be seen with 
the higher standard deviation. The enrichment, however, is much higher than observed in the samples, so the threat to the analytical performance of the samples is minimal.

Table 49: Analytical performance of IAEA-601 and IAEA-602 for $\delta^{18} \mathrm{O}$ analysis

\begin{tabular}{|c|c|c|c|}
\hline Run No. & Standard & Average scaled ( $\boldsymbol{\delta}$, per mil) & Std. deviation \\
\hline 1 & IAEA-601 & 23.30 & 0.04 \\
\hline 2 & IAEA-601 & 23.30 & 0.08 \\
\hline 3 & IAEA-601 & 23.30 & 0.27 \\
\hline 4 & IAEA-601 & 23.30 & 0.20 \\
\hline 5 & IAEA-601 & 23.30 & 0.16 \\
\hline 1 & IAEA-602 & 71.40 & 1.68 \\
\hline 2 & IAEA-602 & 71.40 & 1.35 \\
\hline 3 & IAEA-602 & 71.40 & 1.16 \\
\hline 4 & IAEA-602 & 71.40 & 1.26 \\
\hline 5 & IAEA-602 & 71.40 & 1.89 \\
\hline
\end{tabular}

Similarly to utilizing two standards of differing enrichment in the determination of the $\delta^{18} \mathrm{O}$ the primary and secondary SRMs for $\delta^{2} \mathrm{H}$ also differed significantly in enrichment. In addition, to ensure similarity in sample handing and the effectiveness of the equilibration process both standards were carried through the same procedures as the samples being analyzed. Each standard was subjected to equilibration with water ${ }_{A}$ and water $_{B}$ in order to determine the nonexchangeable component of the $\delta^{2} \mathrm{H}$. Accepted $\delta^{2} \mathrm{H}$ of the IAEA-CH7 and coumarin are $-100.3 \%$ and $62.6 \%$, respectively. The good short and long-term accuracy and precision in both suggests the applicability of this method to make comparisons over the time required to complete all analyses. In addition the excellent results reflect good sampling, sample preparation and equilibration strategies employed in the current studies. 
Table 50: Analytical performance of IAEA-CH7 and coumarin for $\delta^{2} \mathrm{H}$ analysis

\begin{tabular}{|c|c|c|c|}
\hline Run no. & Standard & Average ( $\boldsymbol{\delta}$, per mil) & Std. deviation \\
\hline 1 & IAEA-CH7 & -100.3 & 0.6 \\
\hline 2 & IAEA-CH7 & -100.3 & 0.2 \\
\hline 3 & IAEA-CH7 & -100.3 & 0.7 \\
\hline 4 & IAEA-CH7 & -100.3 & 0.3 \\
\hline 5 & IAEA-CH7 & -100.3 & 1.4 \\
\hline 1 & Coumarin & 62.6 & 0.8 \\
\hline 2 & Coumarin & 62.6 & 0.6 \\
\hline 3 & Coumarin & 62.6 & 0.2 \\
\hline 4 & Coumarin & 62.6 & 0.8 \\
\hline 5 & Coumarin & 62.6 & 0.7 \\
\hline
\end{tabular}

\subsubsection{IRMS Analysis of Domestic and Foreign Unprocessed Cotton Samples}

\subsubsection{Univariate Comparison of $\delta^{13} \mathrm{C}, \delta^{2} \mathrm{H}$ and $\delta^{18} \mathrm{O}$ Results}

The performance of the SRMs and analytical QC samples translate to the samples, yielding similar precision. Triplicate sampling was utilized for carbon analysis resulting in relatively small standard deviations $(0.02-0.13 \%)$, analogous to the standards.

The determinations of the $\delta^{18} \mathrm{O}$ and $\delta^{2} \mathrm{H}_{\text {nonexch }}$ for the samples were done so following the splitting of the sample and equilibration in sealed environments with two water samples of known, but different isotopic composition. Table 51 depicts the ability to determine the $\delta^{18} \mathrm{O}$ in both environments. The small spread of results for $\delta^{18} \mathrm{O}$ obtained in both water environments result from the sample preparation phase of the analysis. In addition, the differences in $\delta^{2} \mathrm{H}$ obtained with the water standards of different isotope composition $(\Delta=100.00 \%)$ emphasize the influence that environment can have on the ability to obtain accurate assessment of the true hydrogen isotope ratio. The differences in $\delta^{2} \mathrm{H}$ values obtained in the presence of different water and thus water vapor environments is due to the ability of certain functional groups (amino, carboxyl, hydroxyl, and thiol) in some organic constituents in the cotton fiber to exchange protons ${ }^{170}$. The differences in the $\delta^{2} \mathrm{H}_{\text {nonexch }}$ for the domestic and foreign examples presented in 
table 51 address the difference in isotopic ranges that are encountered in different regions of the world that grow cotton crops.

Table 51: Comparison of sample performance when extensive sample preparation is employed

\begin{tabular}{|c|l|l|c|}
\hline Sample ID & Sample no. & $\begin{array}{c}\text { Scale corrected } \\
\left(\delta^{18} \mathrm{O}, \text { per mil }\right)\end{array}$ & $\begin{array}{c}\text { Scale corrected } \\
\left(\delta^{2} \mathrm{H}, \text { per mil }\right)\end{array}$ \\
\hline \multirow{4}{*}{$\begin{array}{c}\text { Florence, } \\
\text { S. Carolina } \\
\text { (Domestic } \\
\text { sample \#1) }\end{array}$} & Water A no. 1 & 29.21 & -19.3 \\
\cline { 2 - 4 } & Water A no. 2 & 29.21 & -19.0 \\
\cline { 2 - 4 } & Water A no. 3 & 29.27 & -19.9 \\
\cline { 2 - 4 } & Water B no. 1 & 29.32 & -25.1 \\
\cline { 2 - 4 } & Water B no. 2 & 29.39 & -25.1 \\
\cline { 2 - 4 } & Water B no. 3 & 29.41 & -25.1 \\
\cline { 2 - 4 } & & $29.30 \pm 0.09$ & $-17.2\left(\delta^{2} \mathrm{H}_{\text {nonexch }}\right)$ \\
\hline \multirow{4}{*}{$\begin{array}{c}\text { China } \\
\text { (Foreign sample } \\
\text { \#1) }\end{array}$} & Water A no. 1 & 24.93 & -43.7 \\
\cline { 2 - 4 } & Water A no. 2 & 24.96 & -43.3 \\
\cline { 2 - 4 } & Water A no. 3 & 24.95 & -47.4 \\
\cline { 2 - 4 } & Water B no. 1 & 25.16 & -48.1 \\
\cline { 2 - 4 } & Water B no. 2 & 24.96 & -48.5 \\
\cline { 2 - 4 } & & 25.28 & $-43.0\left(\delta^{2} \mathrm{H}_{\text {nonexch }}\right)$ \\
\hline
\end{tabular}

The entire foreign cotton sample set was selected and analyzed to serve as a proof of concept for foreign samples (no. 1-13). Figure 54 depicts the variation in $\delta^{13} \mathrm{C}$ for the domestic unprocessed cotton samples. There is no clear pattern for the variation within the U.S. for this number of samples. Although there exists some variation in atmospheric $\mathrm{CO}_{2}$ and the $\delta^{13} \mathrm{C}$ will reflect this, the usefulness of this isotope for provenancing may not be as dramatic as $\delta^{2} \mathrm{H}$ and $\delta^{18} \mathrm{O}$ which more directly reflect climate and geographic variations. The range of the $\delta^{13} \mathrm{C}$ in the foreign samples overlaps, figure 55, that of the domestic samples. This is most likely because all samples are of the same plant type, $\mathrm{C}_{3}$, and there is little variation in atmospheric $\mathrm{CO}_{2}$. The use of $\delta^{13} \mathrm{C}$ within a single plant type has only been reported as a useful comparison when the plants to be distinguished were grown in two different environments - indoors and outdoors ${ }^{157,158}$. 


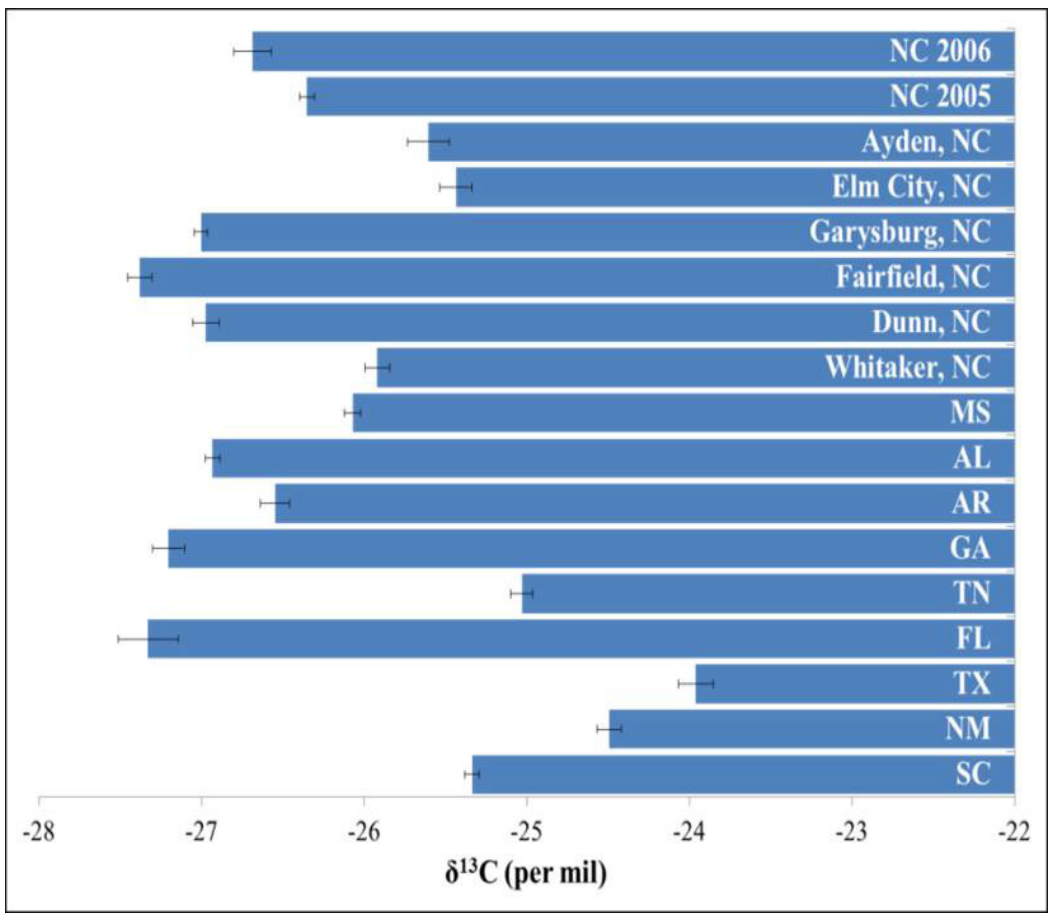

Figure 54: $\delta^{13} \mathrm{C}$ in domestic unprocessed cotton.

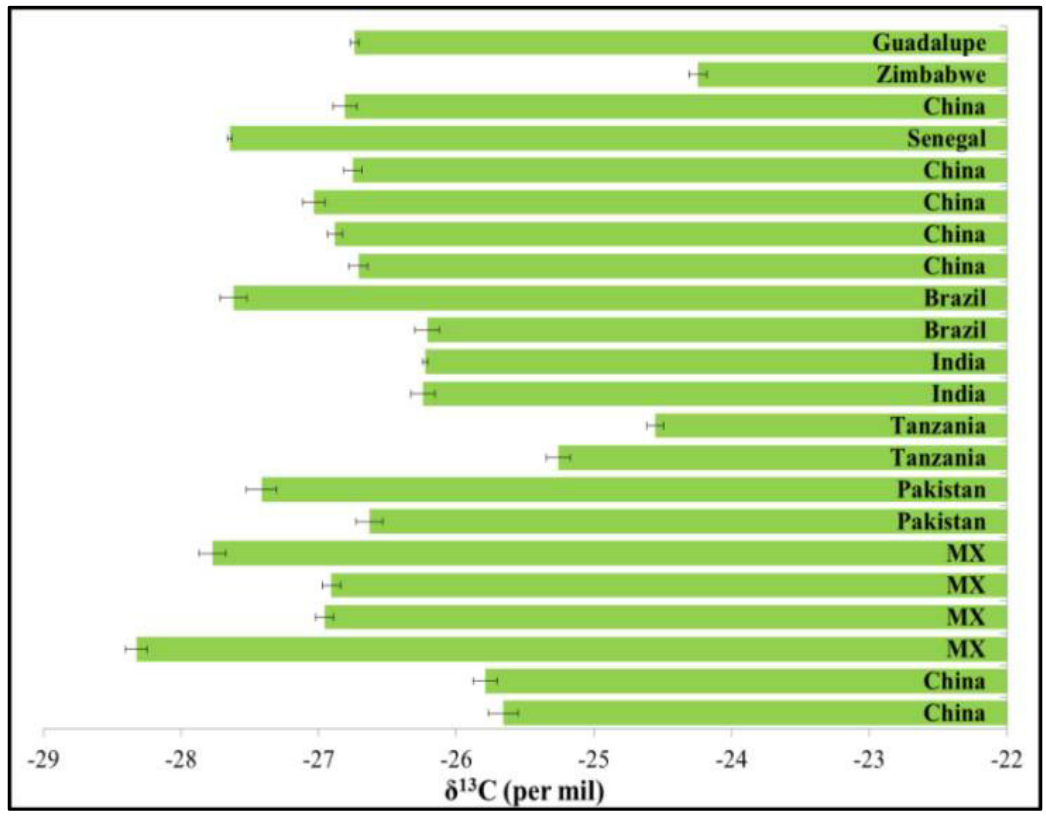

Figure 55: $\delta^{13} \mathrm{C}$ in foreign unprocessed cotton.

When considering isotopic enrichments that are more directly related to geographic region, like

$\delta^{18} \mathrm{O}$, less overlap is observed when comparing foreign to domestic grown cotton, figures 56 and 
57, respectively. However, the variation for the samples from different crop years falls within the variation observed across a region for a single harvest suggesting that the intra-variation of one year is analogous to the inter-variation of other crop years. This is suggested in part because the additional crop years are not consecutive. The additional North Carolina samples are from 2005 and 2006, three and four years prior to the larger sample set. This suggests that, while this is an abridged sample it represents the variation that occurs within and between harvest years for domestic unprocessed cotton. This infers that these trends in stable isotope ratios are stable over time.

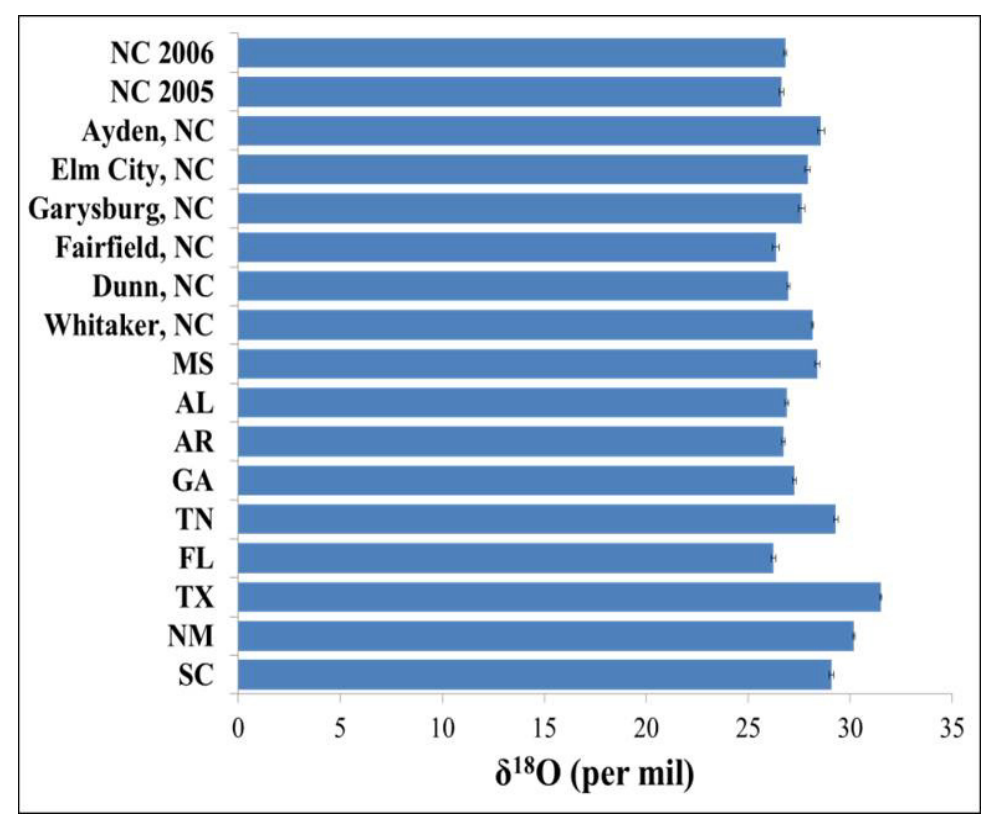

Figure 56: $\delta^{18} \mathrm{O}$ in domestic unprocessed cotton. 


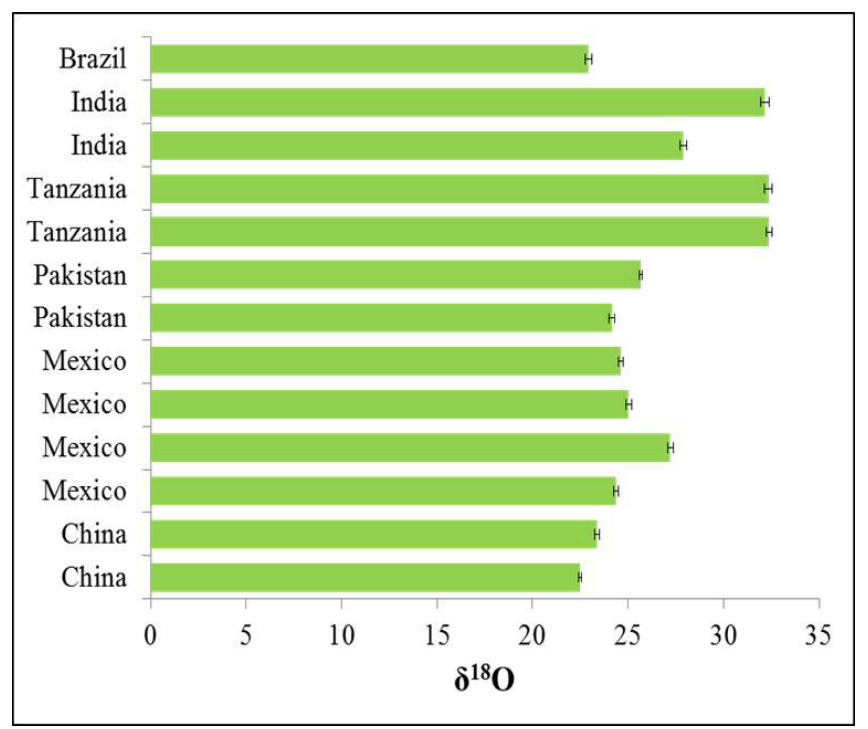

Figure 57: $\delta^{18} \mathrm{O}$ in foreign unprocessed cotton.

In addition, the variation within the North Carolina counties also falls within the range of all the domestic samples analyzed for both $\delta^{18} \mathrm{O}$ and $\delta^{2} \mathrm{H}_{\text {nonexch, figures }} 56$ and 58. However, more variation in the $\delta^{2} \mathrm{H}_{\text {nonexch }}$ can extract additional information regarding the geographic region of origin. This is most likely because water uptake in the plant is the only source of ${ }^{2} \mathrm{H}$, thus establishing a more direct relationship between ${ }^{2} \mathrm{H}$ and the geographic region. The ability to do so has been demonstrated using a small scale study consisting of processed cotton fibers ${ }^{161}$. The trend of the current study is depicted visually in figure 60 in a plot of $\delta^{2} \mathrm{H}_{\text {nonexch Vs. }} \delta^{18} \mathrm{O}$ content. Although clear separation cannot be obtained between, differences between the U.S. and foreign samples can be observed. 


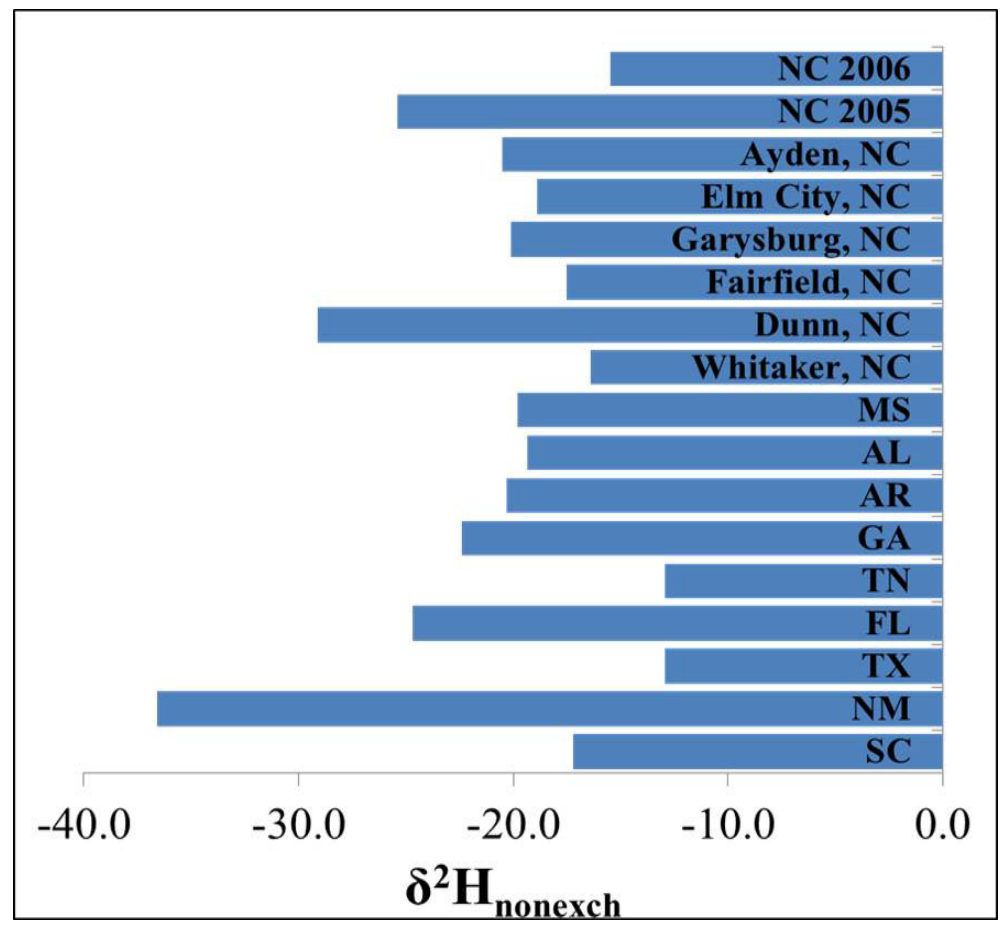

Figure 58: $\delta^{2} H_{\text {nonexch }}$ in domestic unprocessed cotton.

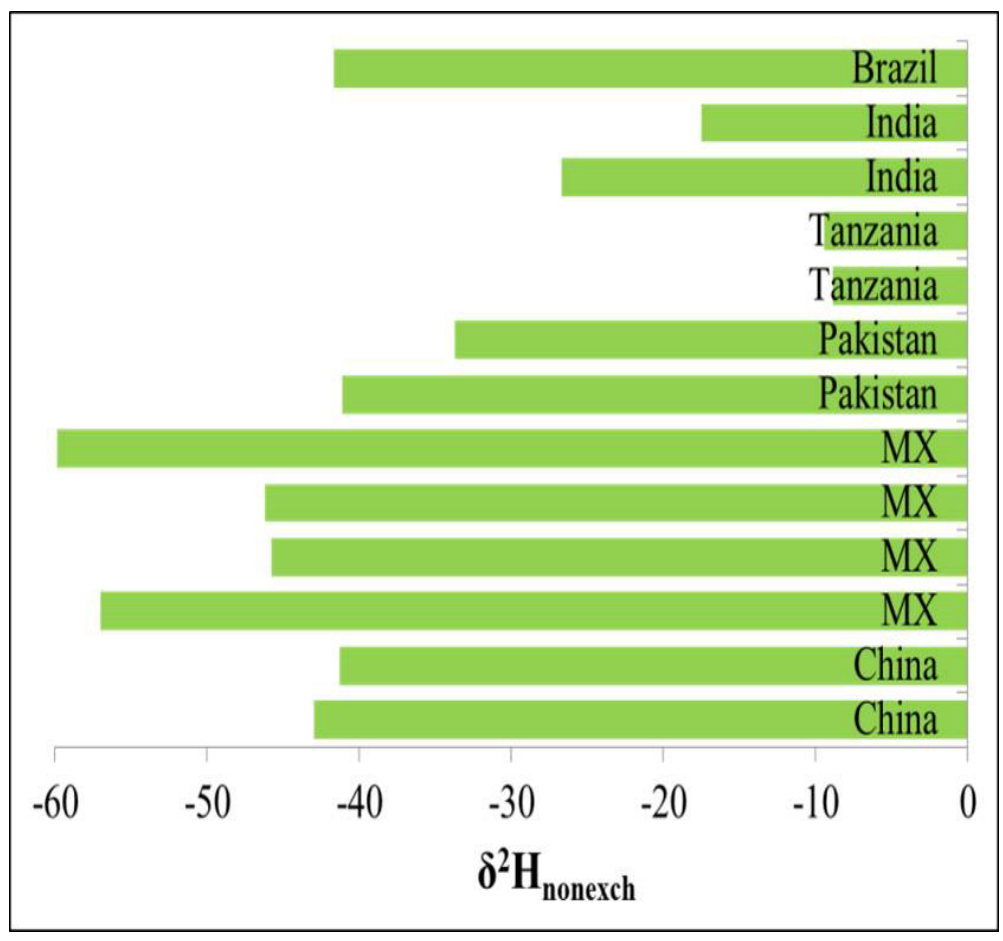

Figure 59: $\delta^{2} H_{\text {nonexch }}$ in foreign unprocessed cotton. 


\subsubsection{Statistical Evaluation}

The enhancement in regional information can be observed when both the oxygen and hydrogen isotopic ratios are used together. As can be seen in the plot depicted in figure 60, the use of these two variables can extract more information regarding the geographic region of origin.

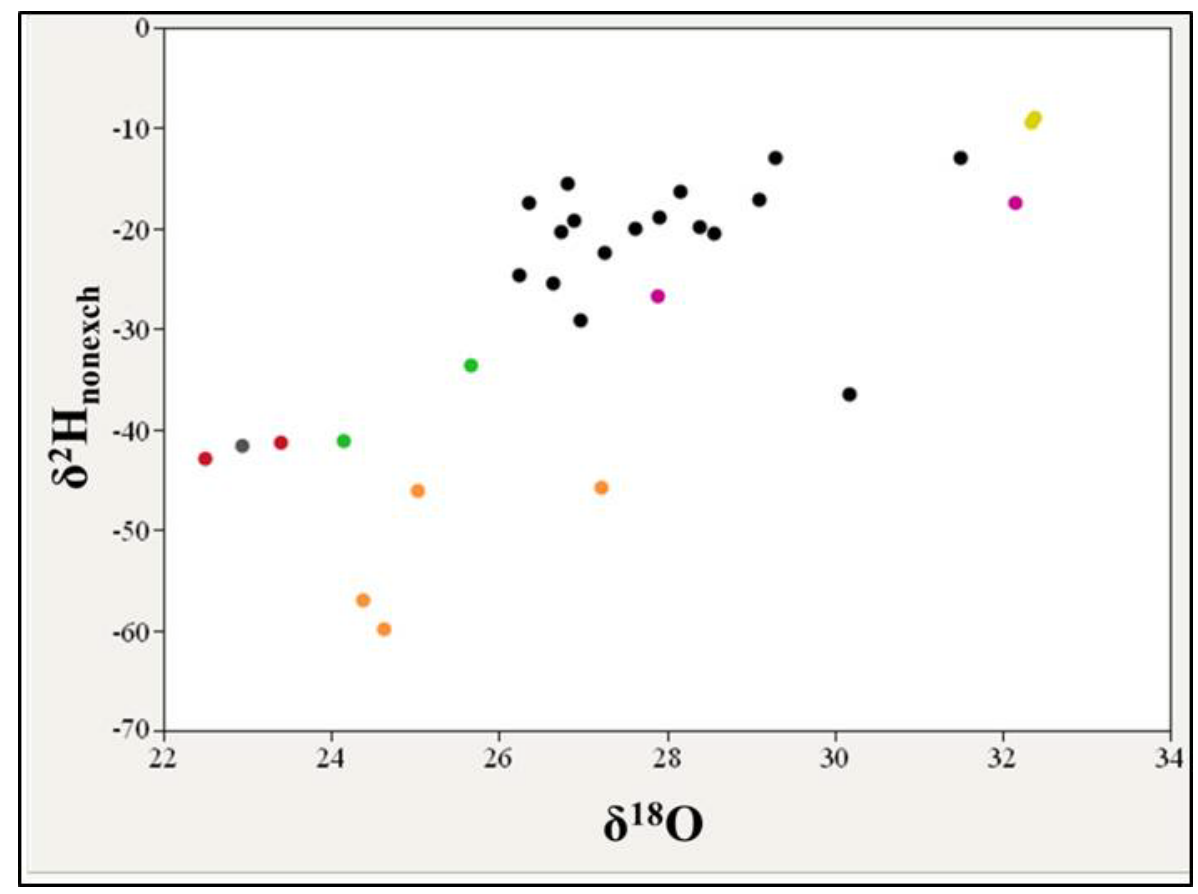

Figure 60: Relationship between $\delta^{2} H_{\text {nonexh }}$ and $\delta^{18} \mathrm{O}$; black $=$ U.S., red $=$ China, orange $=$ Mexico, yellow $=$ Tanzania, green $=$ Pakistan, pink $=$ India and gray $=$ Brazil

To further elucidate trends and interdependency of the stable isotope information, multivariate approaches were employed. PCA was used to visualize these differences observed between all domestic samples and those that have been analyzed of the foreign cotton set (no.1-13). All three isotopes were used to construct the PCA in figure 61. The eigenvectors corresponding to these variables are depicted in figure 62. Each being of similar magnitude and of differing direction implies that all variables contribute to the variation represented in the data. 
The U.S. and foreign samples were separated, however there was overlap within the foreign samples. Because of the number of potential groups (countries in this instance) it is difficult to use a quadrant approach to determine separation. However, the U.S. samples do fall along $y=x$ $(\mathrm{PC} 2$ = PC 1) which serves as a boundary to separate the foreign samples. From this data it is evident that stable isotopes can be used to separate cotton grown in different regions of the world. However, the extent of separation can only be determined with additional sample sets.

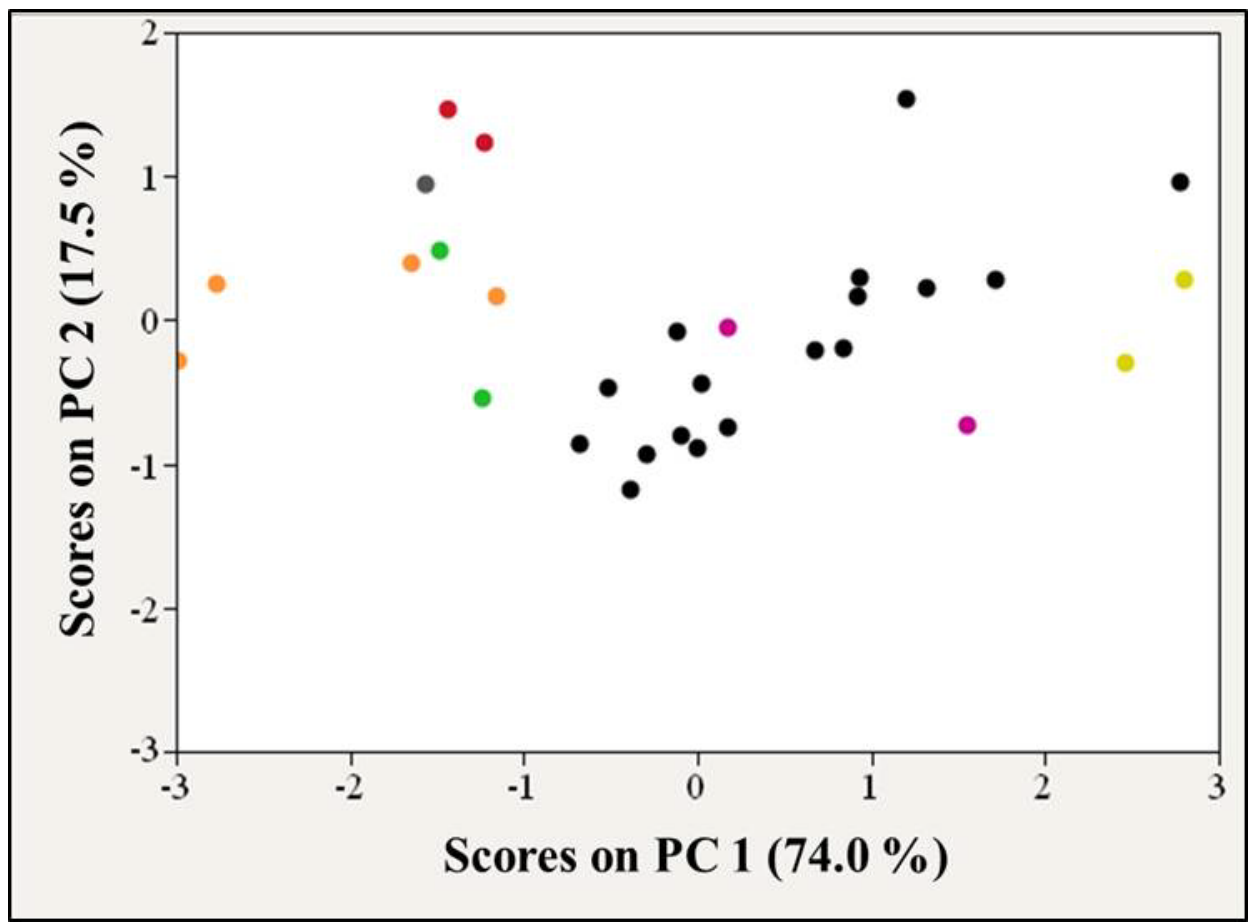

Figure 61: PCA of domestic and foreign unprocessed cotton analysis by IRMS; black = U.S., red = China, orange $=$ Mexico, yellow $=$ Tanzania, green $=$ Pakistan, pink $=$ India and gray $=$ Brazil .

In addition, the four samples from Mexico grouped according to the region from which they originated (Chihuahua and Mexicali), which implies that a representative sampling scheme encompassing more cotton growing regions (similarly to what has been accomplished with the U.S. sampling) is necessary in order to determine the inherent variation between different geographic regions and determine if large countries like the U.S. are comprised of more than one 
region on the basis of sample composition. The variation within a single country has been demonstrated in previous chapters by elemental analysis of domestic cotton.

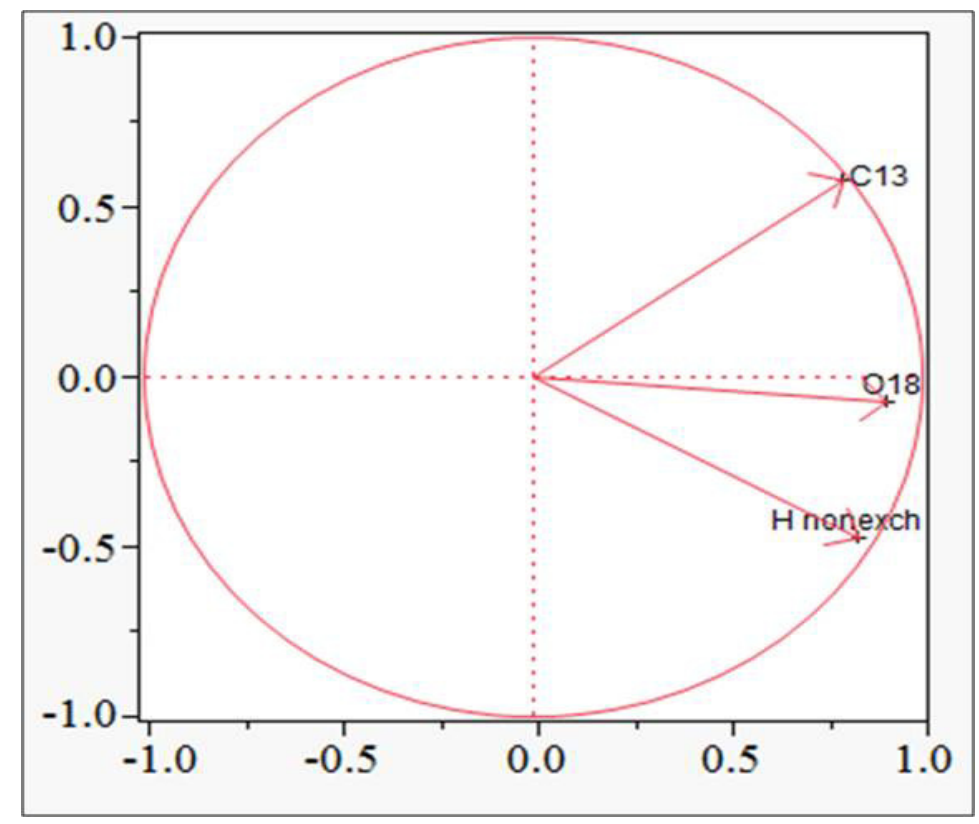

Figure 62: Eigenvectors for variables utilized in Figure 61 PCA; Y $=$ PC 2, X $=$ PC 1.

Because more information was known regarding the origin (location and harvest year) of the domestic samples comparisons are first made with this data. To determine, if trends based on regional climate information such as latitude, longitude, temperature, precipitation as well as the $\delta^{18} \mathrm{O}$ and $\delta^{2} \mathrm{H}$ recorded in that region could be used to predict either or both the stable isotopes of oxygen and hydrogen in the cotton linear regression models were generated. A more detailed explanation of regression modeling is provided in chapter 9 of this dissertation.

The stable isotope information was obtained from the Online Isotopes in Precipitation (OIPC) ${ }^{174}$. Temperature and precipitation data for 2009 were obtained from several databases including the Southeast Regional Climate Center ${ }^{175}$, the National Climate Data Center ${ }^{176}$ and the Oregon Climate Services ${ }^{177}$. Because the temperature and precipitation can vary throughout the year only the months during the cotton growing season (180-200 days) were used to obtain the average 
temperatures and precipitation data used in the model. Since the growing season varies slightly from the southeast to the southwest U.S., the USDA Field Crops Usual Planting and Harvesting Dates 2010 report $^{178}$ was used to determine the growing season dates for each state in 2009 .

The model was generated by first employing a screening technique to determine the usefulness, if any, of the following variables: temperature ${ }_{\text {average }}$, precipitation ${ }_{\text {average }}$, latitude, longitude, $\delta^{2} \mathrm{H}_{\text {region }}$ and $\delta^{18} \mathrm{O}_{\text {region. }}$. The model was built in the forward direction, considering the addition and interaction of each variable singly and together with all other variables in order to optimize model performance. The model was optimized to yield the smallest error in predictions (RMSE value) while accounting for the most variation present in the data $\left(\mathrm{R}_{\mathrm{sq}}\right.$ value). The model generated predicted $\delta^{18} \mathrm{O}$ with the most accuracy when latitude, longitude, precipitation and temperature information of the region were used. Figure 63 depicts the actual vs. predicted $\delta^{18} \mathrm{O}$ for the model. The model accounted for $98 \%$ of the variation $\left(\mathrm{R}_{\mathrm{sq}}=0.98\right)$ and had a very small error estimate of 0.291. Good predictive abilities were obtained, well within the $95 \%$ C.I. region as defined by the red dotted line, with most data falling alongside $\mathrm{y}=\mathrm{x}$.

Because only state information was known, only one sample from North Carolina was included in the model.no matter the sample selected, predictive performance did not change. When all North Carolina samples were included in the regression model a $\mathrm{R}_{\mathrm{sq}}=0.55$ and a $\mathrm{RMSE}=1.042$ was obtained. Based on the information currently known for the samples, model accuracy is better without the extra North Carolina samples. 


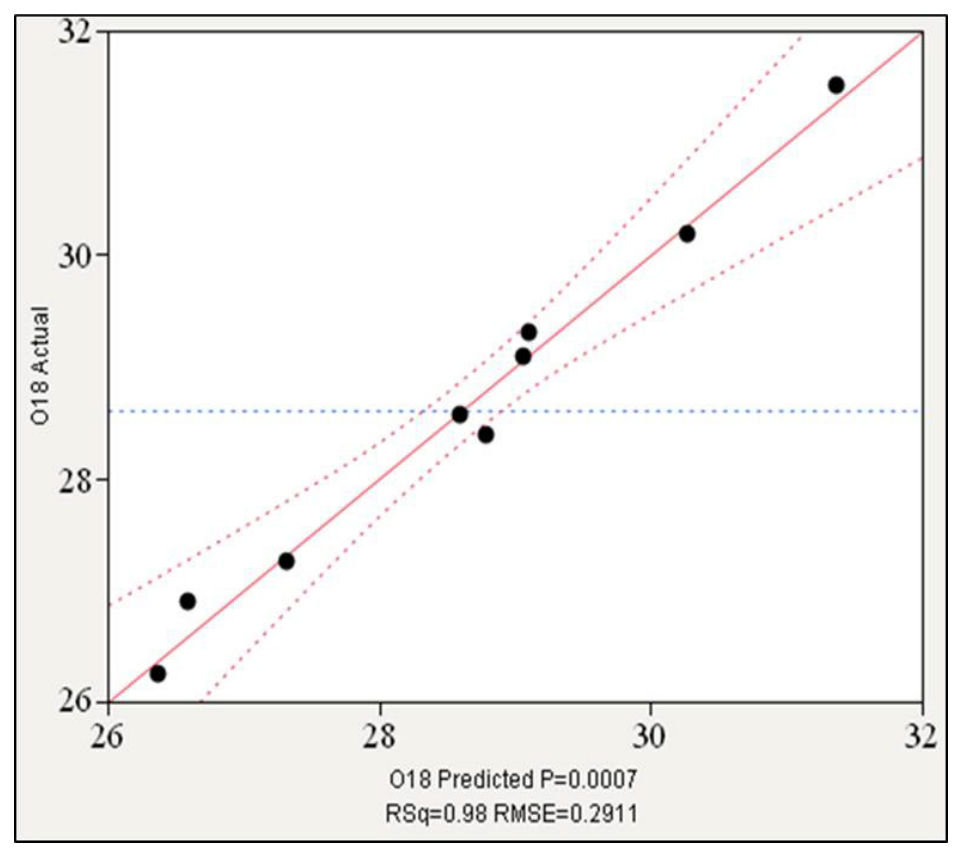

Figure 63: Regression model performance for $\delta^{18} \mathrm{O}$ prediction.

Similarly, a linear regression model was also generated to determine the ability to predict $\delta^{2} \mathrm{H}_{\text {nonexch }}$ from regional information. The same guidelines were used for the $\delta^{2} \mathrm{H}_{\text {nonexch }}$ model generation as in $\delta^{18} \mathrm{O}$. The $\delta^{2} \mathrm{H}_{\text {nonexch }}$ was predicted with the most accuracy using latitude, $\delta^{18} \mathrm{O}_{\text {region, }}, \delta^{2} \mathrm{H}_{\text {region }}$ and temperature information was used. This resulted in a $\mathrm{R}_{\mathrm{sq}}=0.76$ and a RMSE $=4.45$, figure 64 .

The predictive abilities of this model are not very good, reiterated by the spread of data across the broad 95\% C.I. region. This model could be improved with the inclusion of more samples. The spread of $\delta^{2} \mathrm{H}_{\text {nonexch }}$ in the domestic samples is much larger than that of the $\delta^{18} \mathrm{O}$ which could account for some of the error to extrapolate the abilities of the model with few data points. 


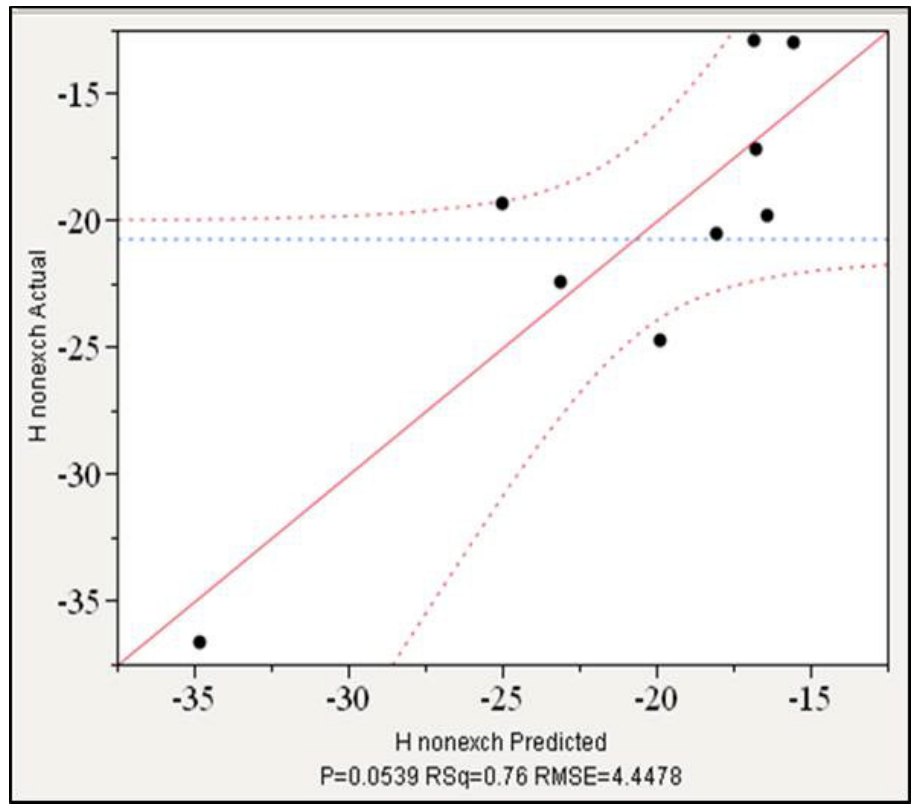

Figure 64: Regression model performance for $\delta^{2} H_{n o n e x h}$ prediction.

\subsection{Discussion and Conclusions}

The use of IRMS for unprocessed cotton analysis has been demonstrated as an analytical approach that can detect differences in samples that can be attributed to differences in geographic regions. The results demonstrate that the variation observed between domestic and foreign samples can be represented using carbon, hydrogen and oxygen information, or just the two latter elements. This study, on a small scale, addresses the feasibility of using this technique, coupled with regional information, in order to generate predictive models to determine the stable isotope ratios, particularly hydrogen and oxygen, in unprocessed cotton. The regression models built suggest that stable isotope models in cotton (at least for the U.S.) can be determined without sample analysis. The ability to model trends in this manner is important for forensic applications, as it allows comparisons to be made without sample preparation, laboratory analysis and extensive data interpretation. This information has the potential to be very useful in accomplishing the geographic provenancing of unprocessed cotton however additional samples and studies are required in order to assess the strengths in these relationships. 


\subsection{EVALUATION OF MULTIVARIATE STATISTICAL APPROACHES FOR GEOGRAPHIC PROVENANCING OF UNPROCESSED COTTON}

\subsection{Overview}

Geographic provenancing is an important tool for determining or authenticating the origin of a material. It can be used for both environmental and forensic applications. The focus of this chapter further pursues the evaluation of geographic provenancing for the purpose of reducing or eliminating the presence of fraudulent unprocessed cotton commodities entering U.S. ports. Geographic provenancing can be accomplished by several methods: determining the concentration of particular elements, or by utilizing the stable isotope ratios to elucidate regional information.

The current studies compare the applicability of both techniques listed for accomplishing the geographic provenancing of unprocessed cotton. While literature reports both techniques are capable of making predictions or forecasting models for geographic provenancing, this research compares both techniques for the same matrix, utilizing the same samples.

The present work evaluates the long-term stability of the approaches, as well as their performance when considering several different types of multivariate approaches to model and interpret the analytical measurements.

\subsection{Introduction}

Statistics refer to "the branch of mathematics that deals with the collection, organization, analysis, and interpretation of numerical data ${ }^{179}$ " Within statistics there are also several branches that indicate the manner in which statistics can be integrated into various fields. Statistics, as it pertains to chemistry is known as chemometrics. Chemometrics is then defined as "the chemical discipline that uses mathematical, statistical and other methods employing formal logic to a) to 
design or select optimal measurement procedures and experiments and b) to provide maximum relevant information by analyzing chemical data ${ }^{180}$."

Chemometrics can be divided into two forms of comparison. These comparisons can either be exploratory or inferential ${ }^{152}$. Exploratory techniques do precisely what the technique implies explore the data. Exploration is typically in the form of graphs, tables and charts that summarize the data. This type of data interpretation is advantageous because it is flexible. No preconceptions of the data are made nor is any information regarding the sample necessary to evaluate the data in this manner. Typically, exploratory statistics result in visual summaries which aide in the simplicity of interpretation. However, the results are then also open to interpretation and outcomes may vary between individuals. Interpretation of this sort relies on the analyst remaining unbiased. In addition, because these summaries are typically visual, no definitive answers are obtained. It is because of these characteristics that exploratory interpretation precedes inferential analysis and is typically a screening approach to making generalizations. Figure 65 displays a typical schematic of data interpretation approaches. The order of steps is important to drawing more accurate, successful conclusions. Describing the data is primarily exploratory, while additional comparisons listed and predictive approaches utilize inferential thinking.

Inferential analysis is a deductive approach. Just as the name implies, this form of chemometrics makes inferences based on the data being compared. Inferences can be the result of hypothesis testing, the use of probabilities and even predictive modeling techniques. The concepts of inferential chemometrics are founded in theory and principles, which lead to definitive answers. However, these principles, while valuable, may only be applicable to ideal circumstances and so conclusions must be thoroughly investigated.

Exploratory and inferential chemometric tools apply to both univariate and multivariate statistical approaches. The actual tools employed in this research have been selected so as to explore 
possible trends and evaluate important aspects of the data so as to determine the extent to which the results address geographic provenancing of unprocessed cotton.

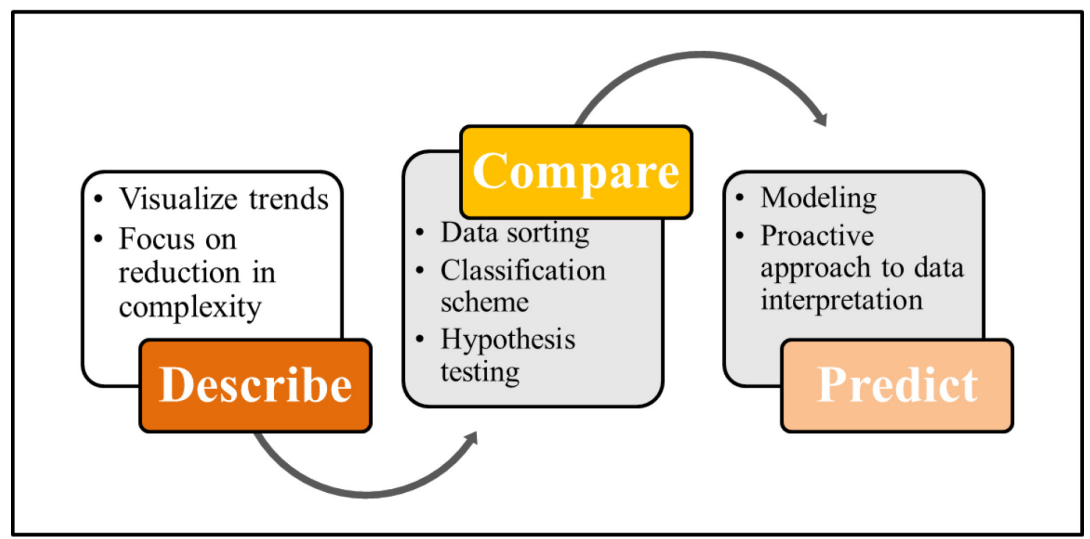

Figure 65: Schematic to conducting interpretation of cotton data.

\subsubsection{Univariate Statistics}

Univariate statistical analysis tools refer to comparisons of data by comparing one variable at a time. The data collected for a single sample is thought of as a vector. A vector comprises a single dimension of values or data. Comparisons can be made within a vector or between vectors. Figure 66 displays the general intention of univariate statistics.

The simplest of univariate measurements involve those pertaining to central tendency - mean, mode and median - as well as those relating to dispersion - variation (range) and standard deviation. The information can be incorporated into bar graphs or box plots and bivariate scatterplots which compare the response of one variable vs. that of another which have been presented in other chapters of this dissertation. The techniques are considered univariate approaches, however when several boxplots or scatterplots of several variables are presented, this may be considered multivariate approaches by some. 
Another univariate approach used to interpret the data, and particularly the distribution of the data is a graphical technique referred to as a histogram. A histogram is a type of frequency plot. The data is distributed in to bins. The height of the bar in each bin corresponds to the frequency of data generated that falls within the range that the bin accounts for. A normal distribution appears to resemble a mean-centered bell curve. Histograms are used to assess the normality of the data. For both univariate and multivariate statistics normality must be established.

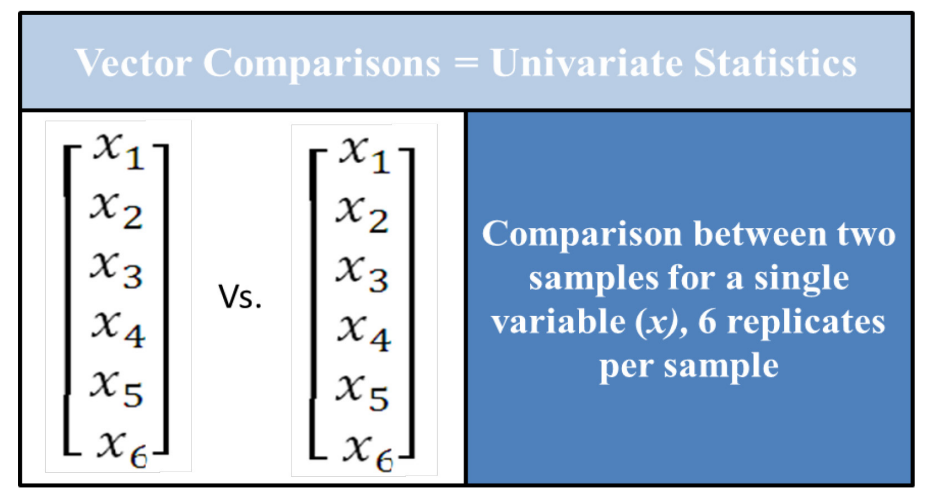

Figure 66: Simplified visual explanation of univariate statistics.

\subsubsection{Multivariate Statistics}

Multivariate statistics is quite the opposite of univariate. This discipline is capable of simultaneously comparing several variables. This is important to determine what interactions exist between the different variables. It has been shown that most variables are not completely independent of one another ${ }^{152}$.

In order to make comparisons, data collected from a single sample is thought of as matrix. Figure 67 presents a pictorial description of inter-sample comparisons. In addition, the measurements of central tendencies and dispersion are not single values; they too are matrices. 


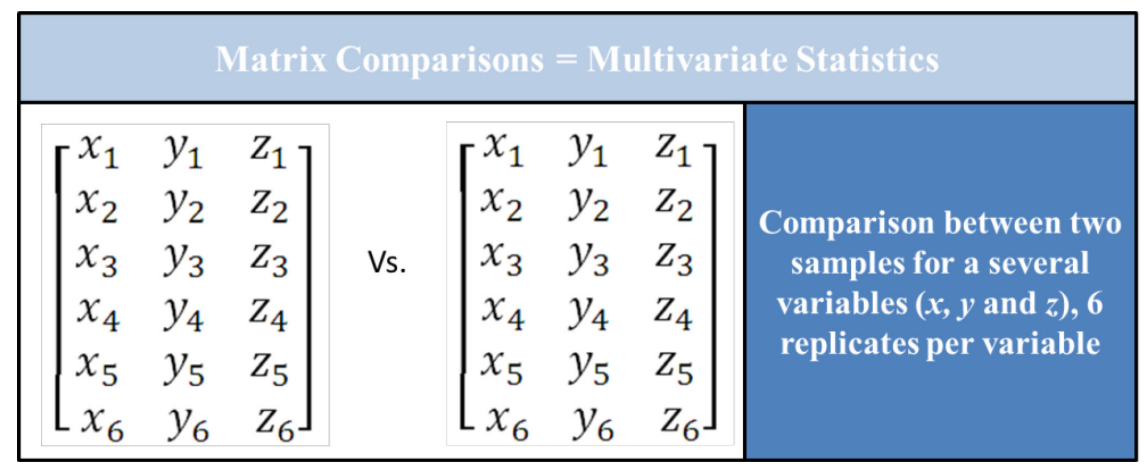

Figure 67: Simplified visual explanation of multivariate statistics.

\subsubsection{Visual Exploration}

There are several exploratory techniques that can be utilized for data analysis when both univariate and multivariate statistics are considered. The discussion presented here is for the purpose of introducing and providing some relevant background on exploratory techniques that have been useful in interpretation of the data generated within the scope of this dissertation. Therefore, not all exploratory techniques are presented.

While univariate techniques utilized in this chapter consists of primarily bar graphs and box plots, the only technique that may require explanation is a box plot. A box plot can also be referred to as a box and whisker plot. This plot serves as a comprehensive summary of the data, described in figure 68. The multivariate exploratory technique utilized throughout this dissertation is PCA. This multivariate approach has been described in section 6.4.3.

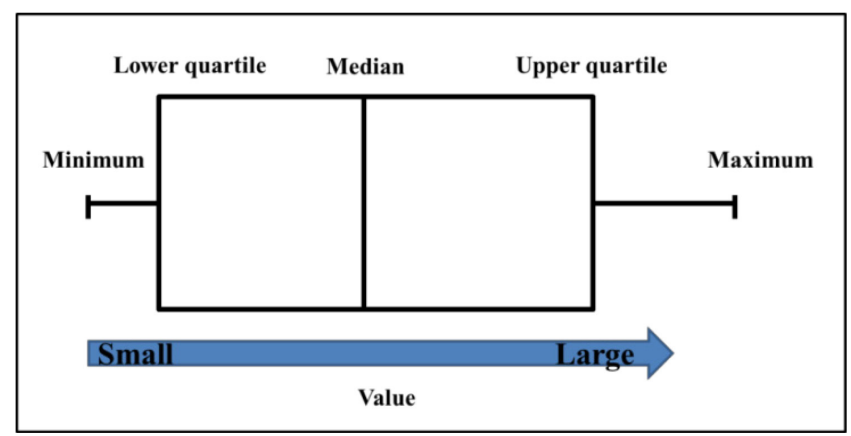

Figure 68: Box plot explained. 


\subsubsection{Predictive Modeling Techniques}

There are several ways in which predictive modeling can be approached. Because the ultimate goal of the research is to associate a cotton sample to a particular geographic region, models that utilize grouping or classification schemes have been employed. Linear discriminant analysis (LDA) has been used extensively in this research. It is considered a supervised technique because information regarding the sample group identity must be known. Using the classes identified by the user, discriminant analysis is designed to maximize the differences observed between the predetermined groups so as to achieve optimal separation, improving classification capabilities.

Two or more groups can be considered in a single LDA model. As the name implies the groups are separated by imaginary linear boundaries. Group assignment is determined on the basis of the proximity to each group. Each sample is assigned to the group to which it is the shortest distance from. The LDA modeling approach can be broken down into several phases. The training phase generates a linear discriminant function (LDF), which is used in the classification of subsequent samples. The LDF, denoted $\mathrm{Y}$, is a linear combination of the variable responses and constants. Each variable contributes to the LDF. Because each variable may have different ranges. For example, if variable A was always be $<1$ and variable B was always $>1000$ then variable $\mathrm{B}$ would normally have more influence in the LDF. In order to prevent one variable from dominating in the LDF scaling can be employed.

$$
\mathrm{Y}=\mathrm{a}_{1} \mathrm{X}_{1}+\mathrm{a}_{2} \mathrm{X}_{2}+\ldots+\mathrm{a}_{\mathrm{n}} \mathrm{X}_{\mathrm{n}}
$$

The LDF simplifies the information from several variables to one value, Y. It is this values that is used in determining the group assignment. Other phases of LDA include testing the model and validating the model. The use of these additional steps may differ in their execution, although are both important to yielding a stable, successful model. Depending on the number of samples 
available to model the trends, only validation may be performed. However, the ability to test the model strengthens the performance, by using additional samples to make adjustments in the function. The training and testing of the model is used to determine the correct classification rate $(\% \mathrm{CC})$, while validation using an independent sample set is used to determine the overall success, or success rate $(\% \mathrm{SR})$ of the model $^{152}$.

Although the main goal of this research was to accomplish geographic provenancing, predictive modeling of the data by other means that address the strength of the relationship between variable information (elemental or stable isotopic in nature) and geographic origin. Regression analysis is not a classification technique. Regression analysis is used to predict one variable from the responses of one or several other variables ${ }^{152}$. Simply put, regression analysis measures the relationships between variables. The error in determining these relationships is determined by a parameter referred to as the root mean square error (RMSE); the smaller the RMSE, the lower the potential for error. Typically an RMSE $>1$ is desired. In addition to the RMSE, other parameters are used to establish the validity of the model. The R-square value, which ranges from 0-1 determines the $\%$ of data represented by the data set. 


\subsection{Materials and Methods}

\subsubsection{Description of Samples}

Samples were obtained from several agencies and industrial companies involved in the production of textile goods. The ability to obtain cotton samples that meet such specific criteria allows for the evaluation of different growing, harvest and environmental conditions that could influence the accuracy of geographic provenancing.

\subsubsection{Domestic Sample Sets}

\subsection{Influence of Crop Harvest Method Sample Set}

In the U.S. there are two main methods by which cotton can be harvested. The methods are referred to as the Stripper and Picker methods, respectively. The Stripper method is a harsher method, stripping the cotton from the plant before the boll has completely opened. While this approach does have the advantage of an early harvest, the more harsh treatment of the cotton can introduce a significant amount of plant material into the crop in addition to damaging the fibers. Both of these factors can influence the resulting elemental profile of the cotton.

The second method, the Picker method, is used once the boll has completely opened. In addition, any plant material that may have been inadvertently collected during the harvest is separated from the cotton and removed by special machinery used in the harvesting process. This method does not damage the fibers significantly.

The samples used to compare these two harvest methods were obtained from two cotton fields in Lubbock, TX. The same harvesting equipment, both Stripper and Picker, were used in each field. The fields from which the cotton originated from were located on two different farms in the town. 


\subsection{Influence of Sample Storage Technique Sample Set}

The manner in which the cotton samples are stored also provides an additional opportunity for environmental contributions to inaccurately influence the elemental profile. To evaluate this potential threat to geographic provenancing a study was conducted to determine what influence, if any, there is from the method or container used for sample storage. Samples that were handpicked by the Regional Breeders Testing Network (RBTN) from the 2009 harvest in Lubbock, TX were used in this study. The samples were selected because they were handpicked and also because specie variation was known. This allowed for more control in variables, minimizing possible variation within each sample.

The sample ( $\sim 10-20 \mathrm{~g})$ was split into approximately equal mass portions and stored in three different types of storage bag devices. Bags were selected over other types of containers because all samples donated up to this stage in the research were done so in some sort of bag. The bags used in this study were 1) original packaging, 2) brown paper bags and 3) Ziploc bags. The bags were purchased in bulk so that all bags used came from the same box.

The samplers in the above described storage were then placed in a box, sealed and stored in a cabinet. Samples went into the box on November 19, 2009 and were removed for sampling and pellet preparation on November 19, 2010.

\subsection{Intra and Inter-variation in Cotton Bales}

A cotton bale weighs between 375 and $515 \mathrm{lbs}^{131}$. Since the ultimate goal of the research is for the analysis of goods entering U.S. ports it is important to assess potential heterogeneity issues that may arise with samples of different size. Because unprocessed cotton is shipped and transported in bales the sampling of such a source is critical to addressing the aforementioned issue of heterogeneity. Three samples were obtained from a single bale of cotton harvested in Post, TN in 
2009. These samples were split from the bale at Cotton Inc. Two additional samples from other Post, TN 2009 bales were also obtained from Cotton Inc. Information regarding if all bales originated from the same farm was not provided. The samples from bale sources were obtained much after the sampling strategy in 6.3.1.1 was developed.

\subsection{Variation within a Field Sample Set}

While it is hypothesized that growing conditions vary from regions separated by distance, the variation within a field should be addressed. Because several bales can be generated from a single field of cotton the variation within a field can also be used to address the potential for heterogeneity between bales from the same or nearby locations (between bale variation).

In addition, because it is most likely that not only a bale but even a field of cotton has been planted with the same species or variation in species, the samples compared from the same field were of the same variation of species. This was ensured by the RBTN, who provided the samples and also field maps. Each cotton variety was planted in a dedicated location across four transects of a field; resulting in four samples per variety per field. Although several samples from several locations were donated from this RBTN sponsored study, the particular variety employed for this evaluation is known as ARK-0113-17. The sample was obtained from Lubbock, TX.

\subsection{RBTN 2009 Harvest Sample Set}

In addition to the RBTN samples used in the previous section to address heterogeneity of the elemental profiles of the unprocessed cotton, other RBTN samples from the 2009 harvest were obtained. Samples were obtained from throughout the cotton belt. Regions include Keiser, AR, Tallassee, AL, Alexandria, LA, Stoneville, MS, Las Crucas, NM, Florence, SC and Lubbock, TX. To compare the differences in cotton species variety (a.k. cotton sub-type) to present similar elemental profiles, four varieties from Las Crucas, NM were selected. The varieties have been 
identified as ARK-0113-17, AU1326, $02 \mathrm{WK}-11$ and MD25. Additional samples from the locations listed above were also analyzed to increase the sample population and to increase the number of cotton producing states sampled.

\subsection{Frontier Spinning Mills 2010 Sample Set}

Samples were obtained from a variety of sources, including industrial mills that obtained cotton from all over the U.S. cotton belt which would, eventually be spun into yarn at their facility. Frontier Spinning Mills was able to provide several samples from across the southeastern U.S., harvested in 2010. These samples were used to aide in achieving a representative sampling of the U.S. cotton across several years. Table 52 contains sample information from this source.

Table 52: Sample information from Frontier Spinning Mills

\begin{tabular}{|l|}
\hline \multicolumn{1}{|c|}{ Location } \\
\hline Theodore, AL \\
\hline Caldwell, AR \\
\hline Leachville, AR \\
\hline Jay, FL \\
\hline Nashville, GA \\
\hline Glendora, MS \\
\hline Ayden, NC \\
\hline Dunn, NC \\
\hline Fairfield, NC \\
\hline Garysburg, NC \\
\hline Whitaker, NC \\
\hline Bennettsville, SC \\
\hline Clio, SC \\
\hline Yorkville, TN \\
\hline
\end{tabular}

\subsection{Arkansas Cotton Class Office 2011 Sample Set}

Additional samples from Arkansas and Missouri were obtained from the Arkansas Cotton Classing office. Samples selected from this submission were also used to address temporal heterogeneity and to add to a representative sample population to establish a thorough profile of domestic grown cotton. Table 53 contains sample information from this source. 
Table 53: Sample information from the Dumas, AR Cotton Classing Office

\begin{tabular}{|l|}
\hline \multicolumn{1}{|c|}{ Location } \\
\hline Dermott, AR \\
\hline Dumas, AR \\
\hline Lake Village, AR \\
\hline Tiller, AR \\
\hline Winchester, AR \\
\hline Bentonia, MS \\
\hline Calhoun City, MS \\
\hline Dublin, MS \\
\hline Gore Springs, MS \\
\hline Greenwood, MS \\
\hline Gunnison, MS \\
\hline Indianola, MS $(2)$ \\
\hline Itta Bena, MS \\
\hline LuLu, MS \\
\hline Lyon, MS \\
\hline Sherard, MS \\
\hline Vance, MS \\
\hline Vardaman, MS \\
\hline
\end{tabular}

\subsubsection{Foreign Sample Set}

Foreign grown cotton samples were obtained from a Cotton Inc. set of samples provided by U.S. Customs and Border Protection. The sample information has been previously listed in table 46 in section 8.3.1.1.2. Because there were significantly less foreign cotton samples available for comparison, the studies conducted within the scope of this research serve as a proof of concept for the geographic provenancing of foreign cotton and for the comparison of differences detected between U.S. and foreign grown cotton.

\subsubsection{Statistical Software Packages}

In order to compare and asses the data that has been obtained with different elemental analysis techniques as well as for different samples analyzed with a single technique, several statistical software packages were employed. The software packages include JMP 9 (SAS, Cary, North Carolina, USA), JMP 9 Pro (SAS, Cary, North Carolina, USA) and Systat (Systat Software Inc., 
Chicago, IL, USA). Both JMP 9 and JMP 9 Pro were utilized for visual exploration of the data by means of PCA as well as for various modeling techniques. JMP 9 Pro was especially useful for generating pictorial representations of the variations in concentrations observed for elements for domestic samples.

\subsubsection{Parameter Selection for Multivariate Modeling of the Data}

Several statistical tools have been used to analyze the data and detect trends or correlation between either or both the elemental profile and stable isotope ratios to geographic source of origin. Modeling of the data can be accomplished in several manners. In addition to selecting the modeling approach, the manner in which the model is generated is also important. This section details the factors that were utilized to develop each type of model. In addition, reasoning is provided in order to rationalize the selection of parameters.

\subsubsection{LDA}

All samples described in the sub sections of 9.3.1.1 and 9.3.1.2 have been utilized in modeling of the data. Initially, U.S. and foreign cotton were treated separately. The domestic samples were compared utilizing the designated regions observed in the initial PCA (see section 6.4.3.1) of the test sample set. While southeast and southwest initially referred to few states, table 54 contains all states now currently classified using the categories.

Because the development of an LDA model requires the employment of a step-by-step procedure to train and validate the model the selection of samples for each stage is critical. The building of an accurate model relies on the principle that the sampling stage of the research generated a representative sample population from which training and validation sets can be constructed. 
Table 54: Cotton producing states organized by region

\begin{tabular}{|c|c|}
\hline Southeast & Southwest \\
\hline Alabama & Arizona \\
Arkansas & California \\
Florida & New Mexico \\
Georgia & Texas \\
Louisiana & \\
Mississippi & \\
Missouri & \\
North Carolina & \\
South Carolina & \\
Tennessee & \\
\hline
\end{tabular}

To combat the unequal numbers of samples for each indicated region a random sample generator was used to arbitrarily exclude extra samples from the southeast region. This was done to ensure that the sample pool for each region contained the same number of possible data points to be selected for each stage in model development. Each sample had the same probability of being selected for each stage of model development. In addition LDA modeling can be accomplished in two or three stages. It was found in this research that a three-tier approach (train, test, validate) resulted in improved classification rates.

A schematic of the developed LDA modeling approach, figure 69, is depicted. Figure 69 also portrays the breakdown of the number of samples utilized in each tier. Notice that the validation pool is kept completely separate from the training and testing pools. Further improvement of the successful classification rates was accomplished through repetition. Repetition was achieved by generating a single model, saving the LDF generated with the model, and then starting the process over again. The LDF was optimized so that the training and test sets produced the highest correct classification for each group considered. 


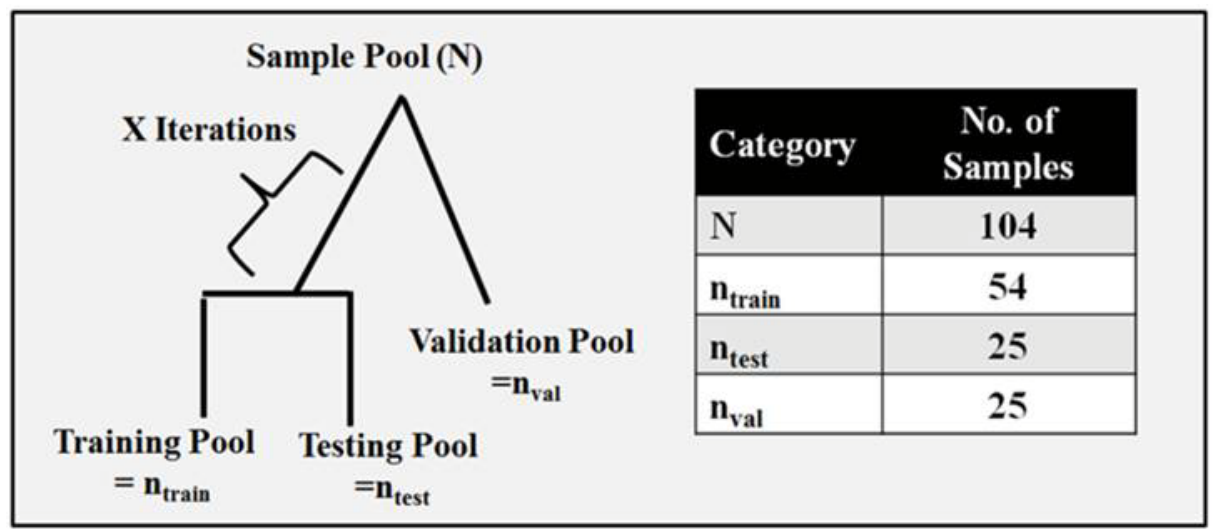

Figure 69: Schematic of LDA approach developed and utilized in this research.

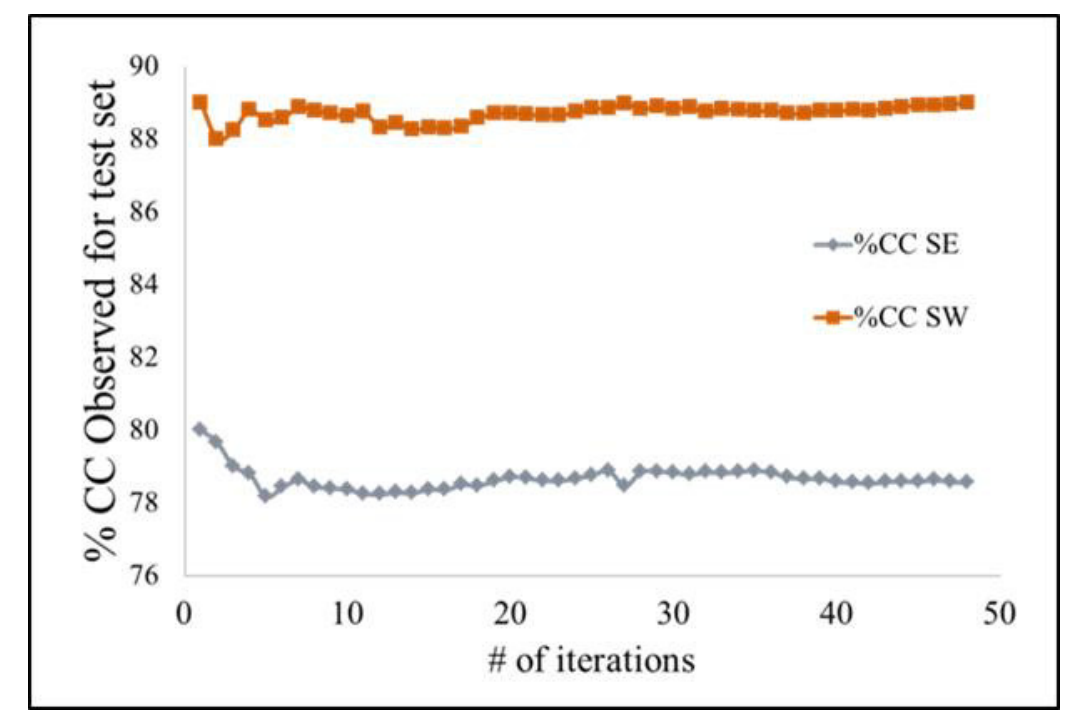

Figure 70: Influence of the number of train/test LDA iterations on \%CC.

A single generation of the model was considered one iteration. As demonstrated in figure 70, as the number of iterations was increased the stability in the correct classification rate $(\% \mathrm{CC})$ steadied. For all the LDA models used in this research fifty iterations were used to generate the model. Each iteration yielded a LDF. The final model was fabricated by compiling each of the 50 LDFs and comparing both classification and success rates. This approach yielded high success rates and demonstrated good model stability and predictability. Both of these factors will be expanded on in later sections. 


\subsubsection{Regression Analysis}

Because linear regression analysis models are used to predict the response, $\mathrm{Y}$, based on numerical values on the independent variable $\mathrm{X}$ or several independent variable, this technique was used to determine if one element concentration could be predicted on the basis of the concentration of another element or several element concentrations similarly to its brief use in chapter 8 . This form of modeling provided insight as to potential relationships between various elements. The strength of these relationships were used to determine trends that correlated to geographic regions, i.e. soil type and mineralogy. This information could be used to assess the potential to forecast the geographic origin of unprocessed cotton over long periods of time. This information is important to the evaluation of the applicability of this research to be implemented for real world applications.

Linear regression based models can be generated in several manners. The model was developed using a two-tier approach to provide at first a means to train and then to validate the predictive abilities. Because the ultimate goal was not classification, the number of samples utilized in the model was not limited. The samples were, however, split into the training and validation sets using a random sampling process. In order to sufficiently train the regression analysis $70 \%$ of the data was used to generate the model and $30 \%$ of the data was used to validate the model. This ratio of training-to-validation samples has been reported to be adequate in linear regression analysis $^{152}$.

Following the arbitrary assignment of data to the sample pools, the initial phase in model generation was conducted in a step-wise, forward progression to select regression parameters. Parameters were selected so as to produce the smallest RMSE and $\mathrm{RMSE}_{\mathrm{val}}$ values. The strength

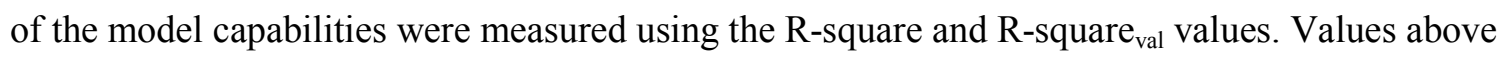
0.80 , which translates to $80 \%$ were considered to be suitable models from which conclusions 
could be drawn. The threshold R-square values imply that the model is able to characterize at least $80 \%$ the data using this model. Upon the completion of building the model, actual vs. predicted concentration plots were generated to determine the overall predictability of element concentrations across the ranges encountered in unprocessed cotton.

\subsection{Results and Data Analysis}

\subsubsection{Addressing Heterogeneity of the Samples}

Univariate statistics are used to address the heterogeneity of the matrix on several levels of the potential sample sources. Heterogeneity has already been considered within a sample and has been accounted for in the sampling strategy for making pellets, section 6.3.1.1. However, to determine the stability of the elemental profile for various sample sources, generalizations can be made regarding the heterogeneity of the material, and thus infers the ability to use elemental profile to establish geographic patterns. Because LA-ICP-MS is the elemental analysis "gold standard $^{3 \%}$ all comparisons pertaining to 9.4 .1 have been made using this technique.

\subsubsection{Variation within a Field}

The variation in a single field was assessed using the concentration of several elements from a single cotton sub-type, figure 71, collected across four transects of a field. Barium was not included in this comparison because the log transformation resulted in a negative value and added complexity to the visual interpretation of the plot. Similarity in concentration was observed for the transects, suggesting that within field variation is minimal. In addition all of these samples were handpicked and thus not subjected to additional environmental contributions during harvest. While the variation in elemental profile at or close to the surface of the soil has been reported in the literate ${ }^{17}$ this does not influence the elemental profile for several reasons. The roots of the cotton plant are significantly below the soil surface and thus the uptake of nutrients is less 
influenced from environmental variables. In addition the elemental profile of the unprocessed cotton is only the result of mobile mineral and non-essential nutrients rather than the total elemental profile of the soil, which is what has been previously presented in the literature ${ }^{17}$.

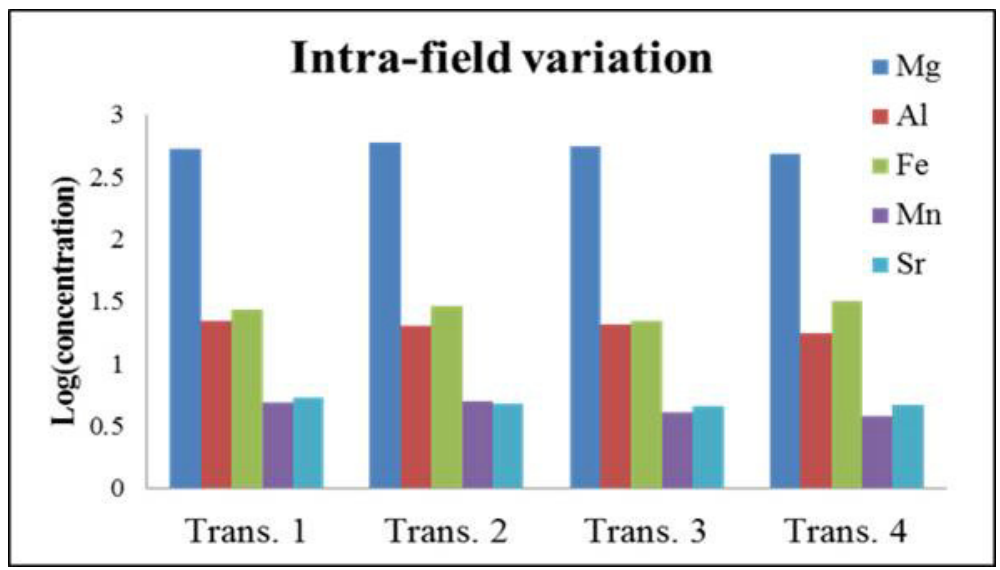

Figure 71: Intra-field variation observed in Lubbock, TX.

\subsubsection{Intra and Inter-variation in Cotton Bales}

Intra and inter-variation of unprocessed cotton in typical bale form, table 55, has been compared. For three samples from a single bale overlap within one standard deviation is observed for the six elements. Although overlap between bales is not observed the difference between bales is smaller than the difference encountered in samples from different regions in the U.S., i.e. NC2005 and AZ2006. From this, it can be concluded that the variation between bales obtained from one location (in this case a single farm or possibly a town) have similar elemental profiles in which the similarity is more than the difference observed between two regions. Assuming that the variation in the U.S. is comparable or less than that observed in other countries it is then possible to use this information to develop a sampling strategy for collecting samples to be analyzed from shipments encountered at U.S. ports. It is hypothesized that the variation present in the elemental profile of the U.S. cotton crops is less than the variation in foreign cotton due to the more stringent agricultural regulations and thus soil quality in the U.S. in comparison to other 
countries. In addition, the extensive use of fertilizers in the country leads to cotton plants of similar of health and thus will participate in mineral and non-essential nutrient uptake to the same degree. Variation then, is introduced by differences in actual soil composition reflected by parent material. Further variation results in differences in the amounts of soil water available to provide a medium to transport these nutrients to the plant.

Table 55: Elemental composition of several cotton bales obtained from Post, TN

\begin{tabular}{|l|c|c|c|c|c|c|}
\hline Sample info & $\mathbf{M g}$ & $\mathbf{A l}$ & $\mathbf{M n}$ & $\mathbf{F e}$ & $\mathbf{S r}$ & $\mathbf{B a}$ \\
\hline $\begin{array}{l}\text { Bale 1, } \\
\text { sample 1 }\end{array}$ & $313.1 \pm 20$ & $10.3 \pm 0.5$ & $0.9 \pm 0.07$ & $8.6 \pm 0.9$ & $0.7 \pm 0.1$ & $1.8 \pm 0.1$ \\
\hline $\begin{array}{l}\text { Bale 1, } \\
\text { sample 2 }\end{array}$ & $287.4 \pm 20$ & $8.7 \pm 0.2$ & $1.0 \pm 0.06$ & $6.1 \pm 0.9$ & $0.7 \pm 0.1$ & $1.8 \pm 0.1$ \\
\hline $\begin{array}{l}\text { Bale 1 } \\
\text { sample 3 }\end{array}$ & $294.2 \pm 20$ & $9.3 \pm 0.3$ & $1.0 \pm 0.1$ & $9.6 \pm 2$ & $0.7 \pm 0.2$ & $1.8 \pm 0.2$ \\
\hline Bale 2 & $272.6 \pm 8$ & $9.9 \pm 0.2$ & $1.0 \pm 0.02$ & $7.2 \pm 0.5$ & $0.8 \pm 0.02$ & $2.1 \pm 0.06$ \\
\hline Bale 3 & $272.5 \pm 10$ & $8.84 \pm 0.4$ & $0.9 \pm 0.04$ & $6.1 \pm 0.4$ & $0.8 \pm 0.06$ & $1.9 \pm 0.05$ \\
\hline
\end{tabular}

\subsubsection{Influences of Crop Harvest Method}

Figure 72 depicts box plots of the variation observed for four variables encountered in the two harvest methods. Overlap between the two methods is observed. Four samples harvested by each technique were analyzed in order to provide a representative comparison of the methods used to harvest the samples. When the spread between the two methods for each analytes is considered in comparison to the concentration observed in the southeastern U.S., table 55 for example, there isn't any overlap.

Ideally, a more thorough evaluation would include an assessment of the harvest methods on the elemental profile of cotton grown in the southeast, these samples were not available. However, because the harvest methods were not known for any other cotton samples and still classification on the basis of geographic region could be accomplished. Harvest method introduces additional 
variation in the elemental profile, although this variation is less than that observed between regions.

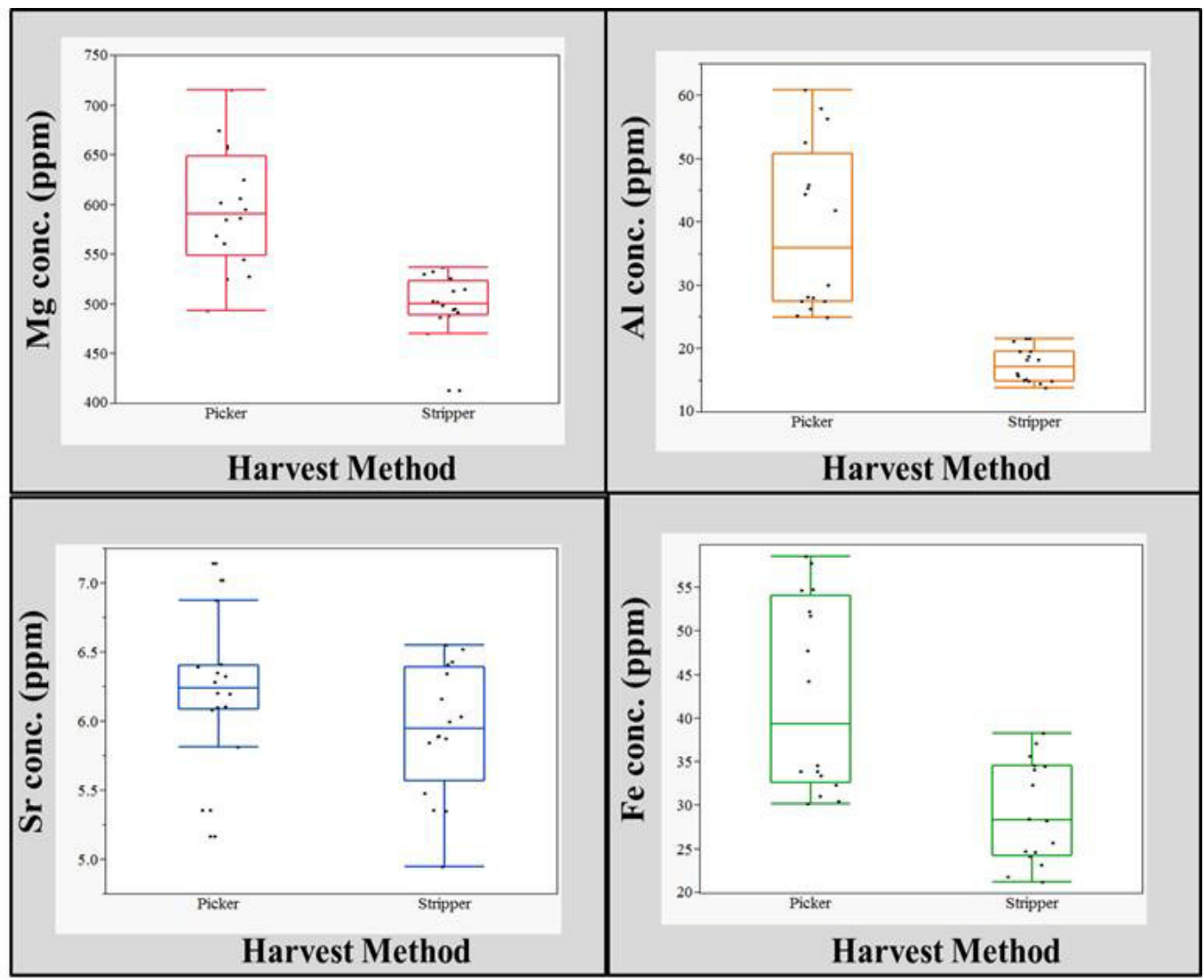

Figure 72: Influence of harvest method on elemental profile.

\subsubsection{Influence of Cotton Variety on Elemental Profile}

Figure 73 presents results from four cotton sub-type varieties from the same field. The elemental profiles are nearly identical. This was observed for other locations considered (S.C. and M.S., data not shown for the sake of brevity). From this it can be concluded that the cotton sub-type does not have a significant, observable effect on variation in elemental profile. This corroborates results for the inter-variation of cotton bales, since several bales are produced from a single cotton field. 


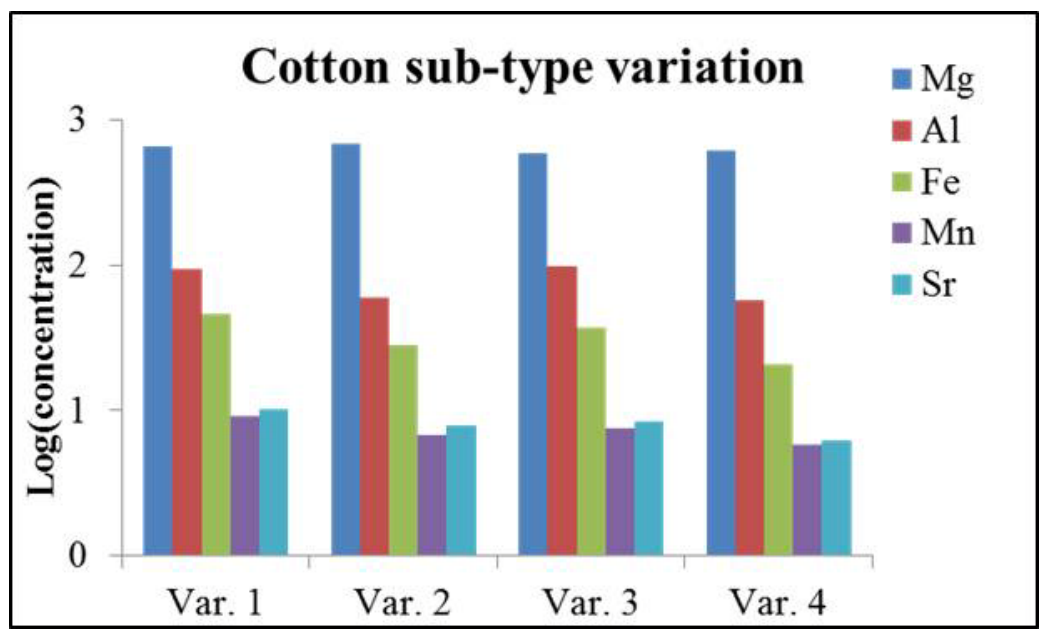

Figure 73: Variation within a field for different cotton varieties.

\subsubsection{Influence of Sample Storage Technique}

The samples submitted to our laboratory were stored in different packages. To ensure that the cotton was not subjected to surface contamination and transfer of elemental information that could influence the resulting elemental profile several methods of storage devices were evaluated as potential sources of heterogeneity. However, from the results for two different samples presented in figures 74 and 75 storage containment did not influence the elemental profiles following one year storage in these bags. Storage in paper or plastic bags did not significantly alter the elemental profile from that which would be obtained if the samples remained in the original packaging. 


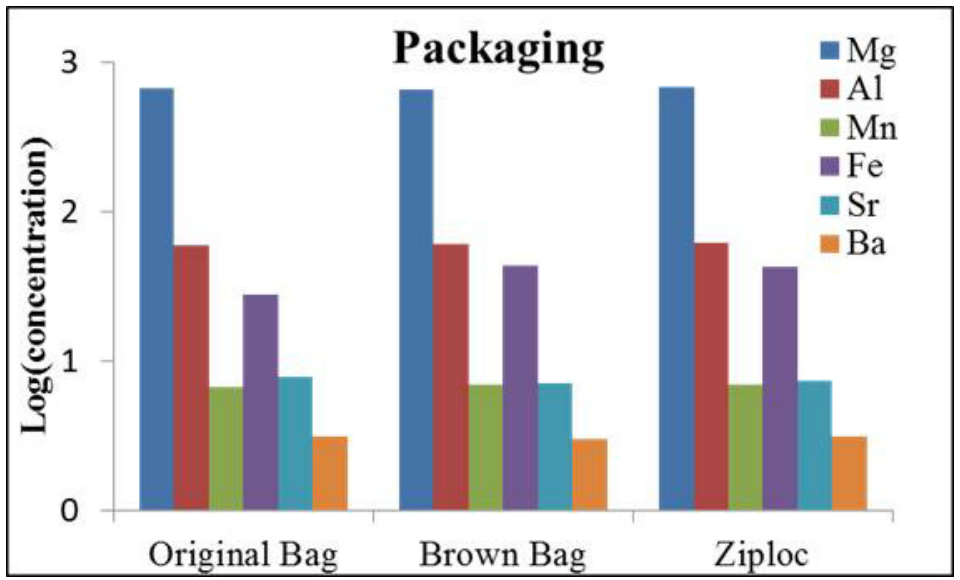

Figure 74: Influence of sample packaging, example 1.

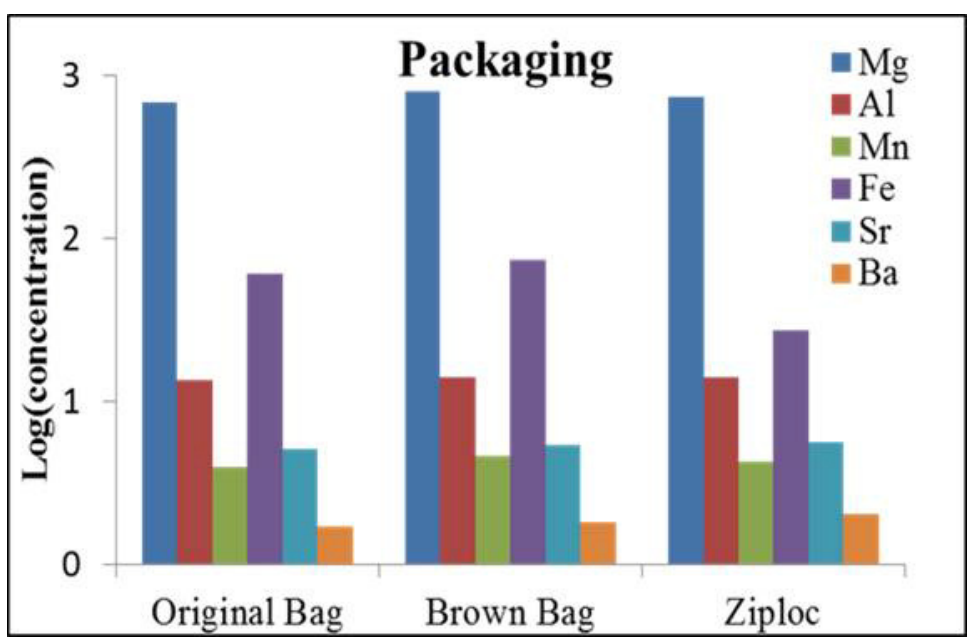

Figure 75: Influence of sample packaging, example 2.

\subsubsection{Geographic Provenancing of Unprocessed Cotton Samples}

In order to make predictive comparisons of the data employing multivariate statistical tools an assessment to confirm the normality of the data was first conducted. Normality was established in several ways to infer a multivariate normal distribution. Multivariate normality was established by determining that each variable was univariate normal and also by establishing bivariate normality between all pairs of variables. If these two statements could be achieved then normality was extrapolated to be multivariate. 
Visual inspection of distribution histograms resulted in using southeast and southwest data as two populations. This was done because a requirement of normally distributed data is that it has to be continuous. For most elements, there was no overlap in the determined calculations. This was also evident when using descriptive features of the histograms, i.e. skewness and excess kurtosis. Skewness is a measure determines how centered the bell curve is. A negative value implies that the data is skewed in the direction of the left tail of the distribution. Excess kurtosis is used to assess the overall shape of the bell curve. A flat topped bell-curve has a kurtosis value $>3$, while a very narrow distribution has a kurtosis value $<3$. Excess kurtosis is then the difference between the kurtosis value of the distribution and 3. The quality of these measurements improved drastically when southeast and southwest U.S. cotton producing states were considered separately.

In addition the Shapiro-Wilks W test was used to establish normality using hypothesis testing. The Shapiro-Wilks test is considered a "goodness-of-fit" test used to determine if the data conforms to the shape of a bell curve and thus is considered to be normally distributed at the particular CI level ${ }^{181}$. The null hypothesis used for this test is that the data presented is normally distributed. At the $95 \% \mathrm{CI}, \alpha=0.05$. Therefore when the test vale (p-value) is greater than 0.05 the null hypothesis is accepted, and the data is considered to be normal. To obtain p-values $>0.05$ the data was transformed using logarithmic tranformations. The transformation also improved the skewness and excess kurtosis as well.

Bivariate normality was established using plots of the variable responses with density elipses at the $95 \%$ CI overlaid. Data shown has been log transformed. A ellipsoid of approximately $45^{\circ}$ to $(0,0)$ was used to estimate bivariate normality. This is true for bivariate populations (math not presented $)^{182}$. Figure 76 displays the scatterplot matrix for all six variables for log transformed concentration values obtained in LA-ICP-OES to demonstrate this theory. While this transform 
was not described in the earlier chapters, it was used to present the PCA data in chapters 6 and 7 of this dissertation.

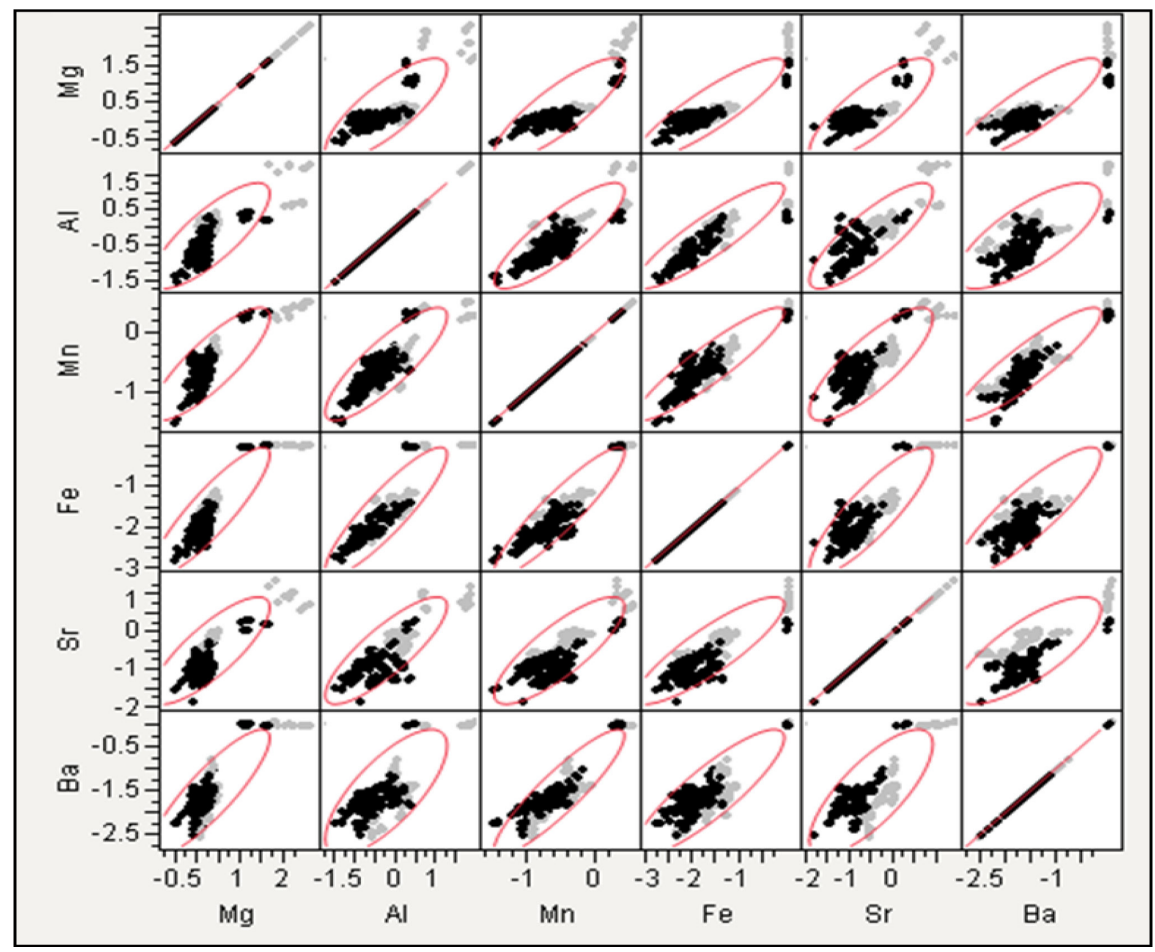

Figure 76: Scatterplot matrix used to estimate bivariate normality.

From this it was determined that following a logarithmic transformation of all analyte data a multivariate normal distribution could be obtained.

\subsubsection{LDA: Classification of Domestic Samples}

The resulting LDA of all elemental analysis techniques is presented. In addition to a visual comparison of the ability to group the data on the basis of geographic differences a table to summarize the LDA performance is presented. The performance is broken down in to correct classification rate and success rate. This corresponds to the predictive abilities of the model for the training and testing phases, as well as the model validation, respectively. The success rate in all instances is used to describe the overall performance of the model. The percentages in 
parentheses refer to the model performance when $\log \left(\right.$ intensity $_{\text {analyte }}$ intensity $\left._{\text {carbon }}\right)$ is used. The other values correspond to the model built using the log transform of the calaculated analyte concentration.

\subsection{LIBS Results}

Some separation between the two U.S. regions is observed in the PCA, figure 77. The data shown in this PCA is also $\log ($ conc), the same data ouput format used in previous chapters. In comparison to the PCA results for the ICP techniques, LIBS has more potential for outliers that do not cluster with the overall data trends. The extensive overlap also made it difficult to determine separation using quadrants or other invisible boundaries. This overlap and wide spread of possible outliers has the potential too, and did affect the modeling abilities of the LDA, with LIBS yielding a lower success rate that either ICP technique.

However, it should also be stated that this is the first attempt on a large scale to provide a method of geographically provenancing unprocessed cotton as an independent method of commodity source identification. Therefore the ability to do this successfully $74 \%$ of the time is still progress.

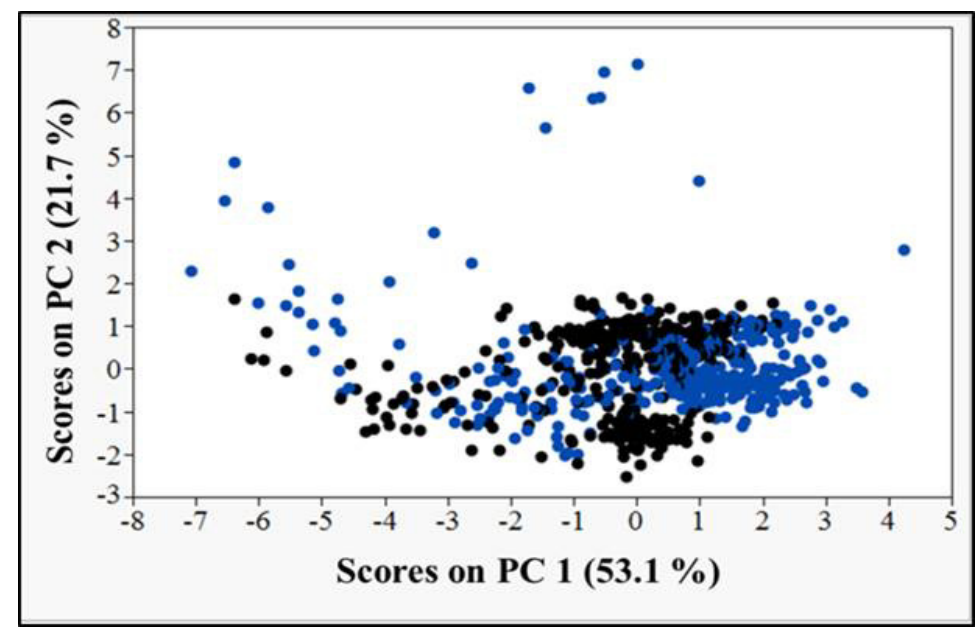

Figure 77: PCA of all domestic cotton samples analyzed by LIBS, blue $=$ southwest, black = southeast. 
Table 56: LDA results of LIBS analysis of domestic cotton

\begin{tabular}{|c|c|c|}
\hline Classifier & Correct Classification & Success Rate \\
\hline Southeast U.S. & $89 \%(85 \%)$ & $74 \%(74 \%)$ \\
\hline Southwest U.S. & $79 \%(79 \%)$ & $75 \%(74 \%)$ \\
\hline
\end{tabular}

\subsection{LA-ICP-OES Results}

Figure 78 presents the PCA of all the domestic unprocessed cotton analyzed using LA-ICP-OES. Interestingly, LA-ICP-OES is also able to detect temporal variation in addition to spatial (geographical) variation as has been indicated in the score plot. LA-ICP-MS and LIBS were not able to separate these attributes using PCA, vertical boundary at PC $1=0$.

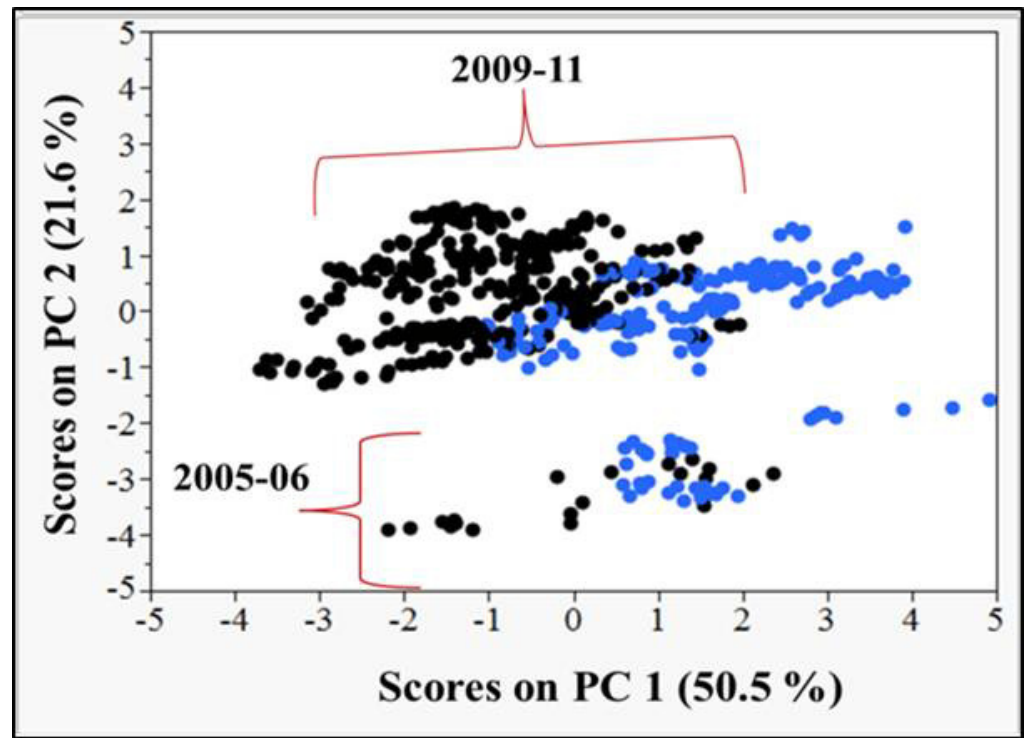

Figure 78: PCA of all domestic cotton analyzed by LA-ICP-OES, blue = southwest U.S., black = southeast.

Table 57: LDA results of LA-ICP-OES analysis of domestic cotton

\begin{tabular}{|c|c|c|}
\hline Classifier & Correct Classification & Success Rate \\
\hline Southeast U.S. & $95 \%(95 \%)$ & $93 \%(92 \%)$ \\
\hline Southwest U.S. & $96 \%(93 \%)$ & $93 \%(90 \%)$ \\
\hline
\end{tabular}


The ability of LA-ICP-OES to classify the data with great success, $>90 \%$, regardless of the data input (intensity vs. concentration) type. The LDA approach utilized here reduces the potential for temporal variation to negatively impact group assignment since data is randomly selected for each step of model development and each sample has an equal probability of being selected. However, for real world use this may be problematic. The LDA modeling approach may only be possible for real-world application with the caveat that the model would require additional testing for model adjustment every $\mathrm{x}$ years, $\mathrm{x}$ to be determined following evaluation in field. While this is not a current problem, the potential threat should be disclosed in order to thoroughly asses the feasibility of the technique to achieve the long-term goal. However, it should also be included that all models would need re-evaluation every $\mathrm{x}$ years in order to adapt to changes in the elemental profile over time.

\subsection{LA-ICP-MS Results}

In addition, LA-ICP-MS is able to elucidate elemental variation between southeast and southwest cotton producing states with little overlap using $\mathrm{y}=\mathrm{x}$ as a diagonal boundary. Figure 79 depicts the PCA of all domestic cotton samples analyzed using this technique.

The sensitivity to geographic variation is translated to great success rates, similar to what has been presented for the other ICP technique. Both of the techniques perform similarly for the classification of domestic unprocessed cotton samples. 


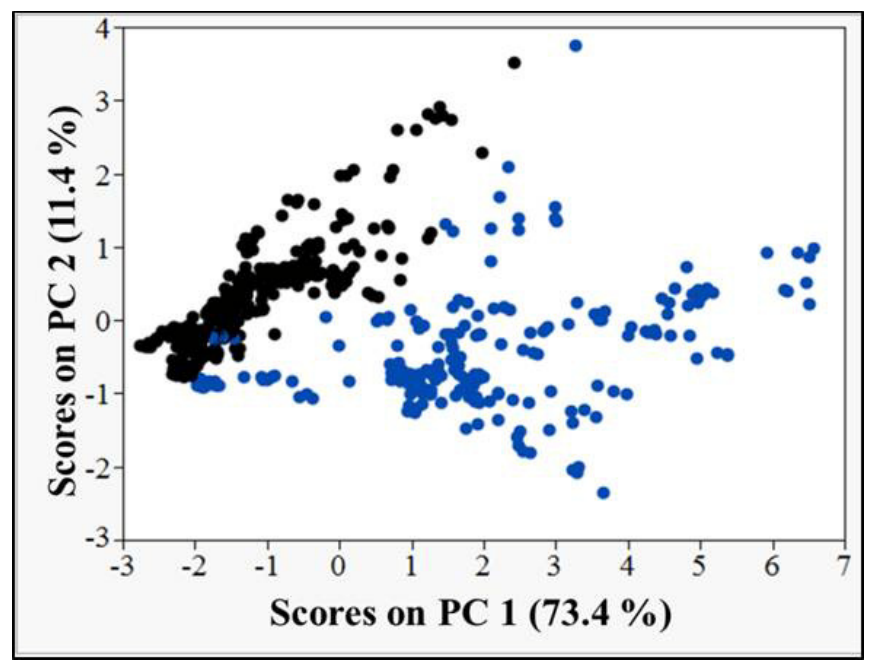

Figure 79: PCA of All Domestic Cotton Analyzed by LA-ICP-MS, blue = southwest U.S., black = southeast.

Table 58: LDA results of LA-ICP-MS analysis of domestic cotton

\begin{tabular}{|c|c|c|}
\hline Classifier & Correct Classification & Success Rate \\
\hline Southeast U.S. & $94 \%(92 \%)$ & $91 \%(88 \%)$ \\
\hline Southwest U.S. & $95 \%(91 \%)$ & $93 \%(89 \%)$ \\
\hline
\end{tabular}

\subsubsection{Addressing the Elemental Profile of the U.S. Using Geographic Maps}

The success of elemental analysis in general to accomplish geographic provenancing is summarized by the variations in a major contributing element $(\mathrm{Mg})$ and a trace element $(\mathrm{Sr})$, the concentrations of which are presented on geographic maps of the U.S, figures 80 and 81 , respectively. The differences in color observed for the two defined regions stresses the differences for the regions. The figures also emphasize of thoroughly evaluating domestic elemental profile. Because of the smaller sample population of available, authentic foreign grown cotton, the U.S. is used as a model cotton producing country. Variation encountered here is hypothesized to be applicable to the potential variation encountered in other regions of the world. From this it is important to keep in mind that large cotton producing countries may present similarities in that two (or more) regions may be required to account for the differences in regions 
within a country. This has somewhat been confirmed in chapter 8, with PCA separation achieved for the two regions in Mexico using $\delta^{2} \mathrm{H}, \delta^{13} \mathrm{C}$ and $\delta^{18} \mathrm{O}$ information.

The current research provides a strong, in-depth foundation for the geographic provenancing of unprocessed cotton utilizing the analysis of domestic cotton as a prototype to determine feasibility of the application. Modeling and other multivariate analysis that considers foreign cotton should be considered preliminary.

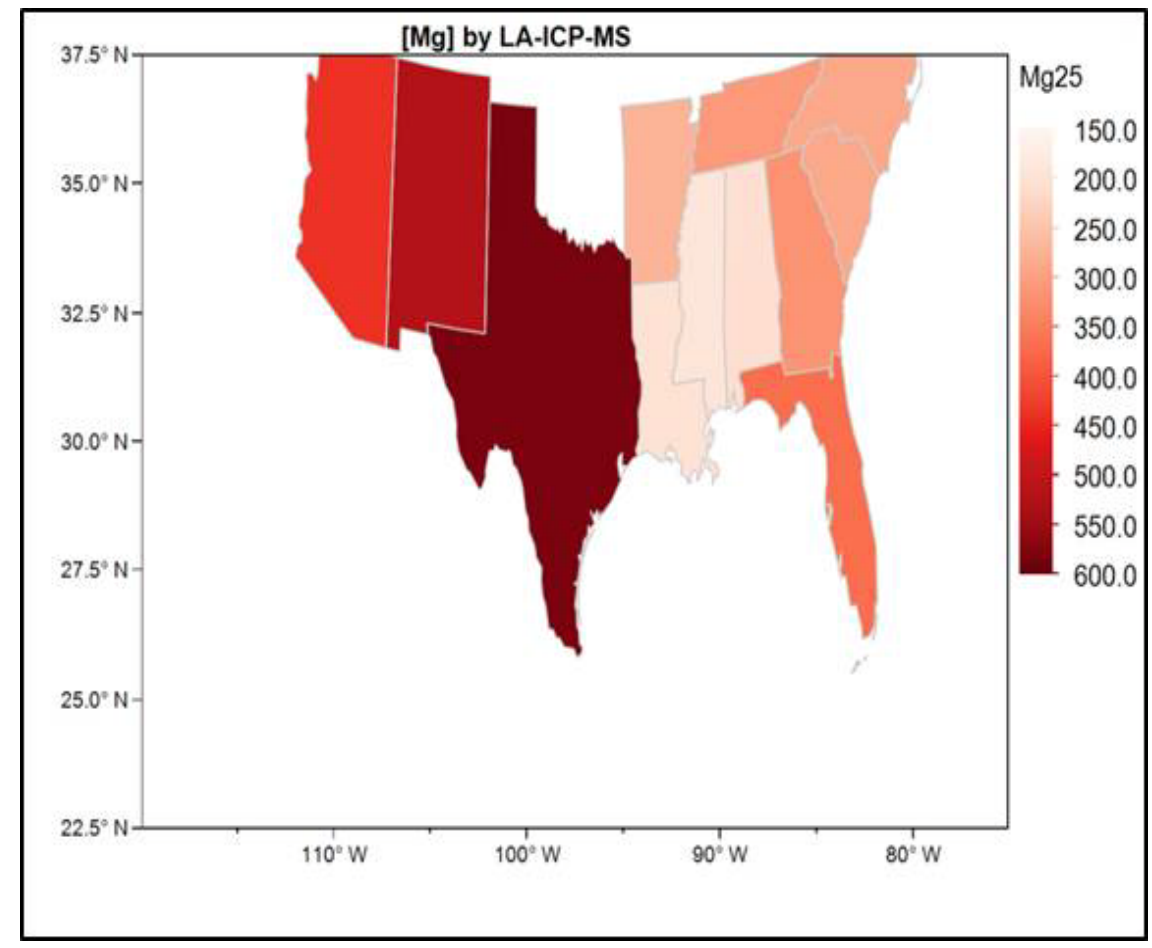

Figure 80: Geographic representation of $[\mathrm{Mg}]$ obtained in cotton grown across the cotton belt. 


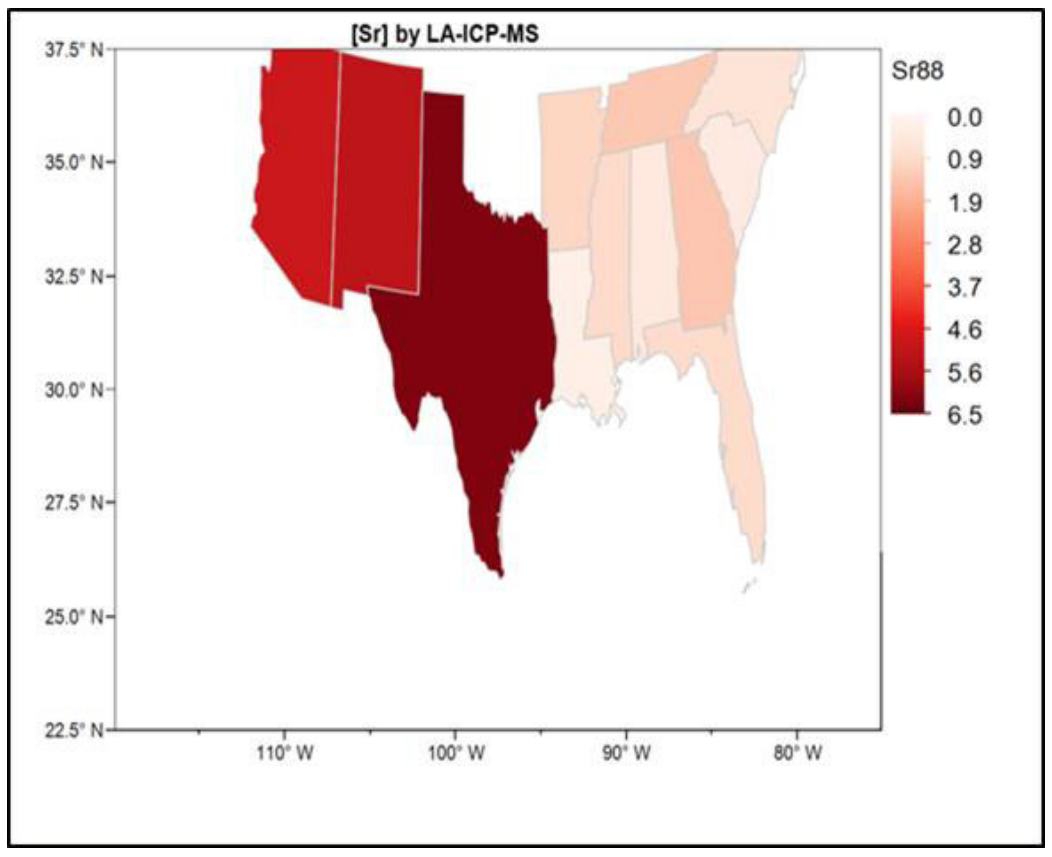

Figure 81: Geographic representation of [Sr] obtained in cotton across the cotton belt.

\subsubsection{LDA: Classification of Foreign and Domestic Samples}

In addition to domestic classification, LDA was also conducted to compare the U.S. and foreign cotton samples together. All samples were included in the PCA to assess variation observed, however group size was limited in the generation and subsequent validation of the LDF generated. The number of non U.S. samples limited the number of southeast and southwest samples that could be included. The U.S. was treated as two populations since this assumption has been confirmed through normality assessments and previous LDA modeling. Because of the small population of foreign samples, regardless is actually from one or more populations, were grouped together as "foreign" so modeling could be performed.

The overall approach to LDA was still used. The model was trained using $\sim 50 \%$ of the data, following testing and validation, each using $\sim 25 \%$ of the data. Southeast and southwest U.S. sample pools were generated using random number assignments. 


\subsection{LIBS Results}

Figure 82 presents a PCA of the entire domestic and foreign cotton is too complex in overlap to determine any trends that pertain to geographic differences.

The complexity is also seen with several misclassifications of the three groups in the LDA. Table 59 only displays the success rates of the LDA. The table can be read as actual rows vs. predicted columns. Since misclassification of southeast and southwest samples does not affect the country or overall region assignment the concern in performance is for misclassifications between U.S. and non U.S. cotton. When this comparison is made, $25 \%$ of non U.S. cotton is misclassified while $20 \%$ of the U.S. cotton is misclassified. Although further improvements are expected with the inclusion of additional samples, it is still hypothesized that LIBS will yield misclassifications of $\sim 20 \%$, similarly to what has been presented for the classification of domestic cotton using LDA. This conclusion has been drawn from the assumption that the analysis and modeling of foreign cotton will result in similar geographic provenancing capabilities as achieved with domestic cotton.

Table 59: LDA results for domestic and foreign cotton analysis by LIBS

\begin{tabular}{|l|c|c|c|}
\hline Classifier & Non U.S. & S.E. U.S. & S.W. U.S. \\
\hline Non U.S. & $75 \%$ & $10 \%$ & $15 \%$ \\
\hline S.E. U.S. & $11 \%$ & $75 \%$ & $14 \%$ \\
\hline S.W. U.S. & $9 \%$ & $11 \%$ & $80 \%$ \\
\hline
\end{tabular}




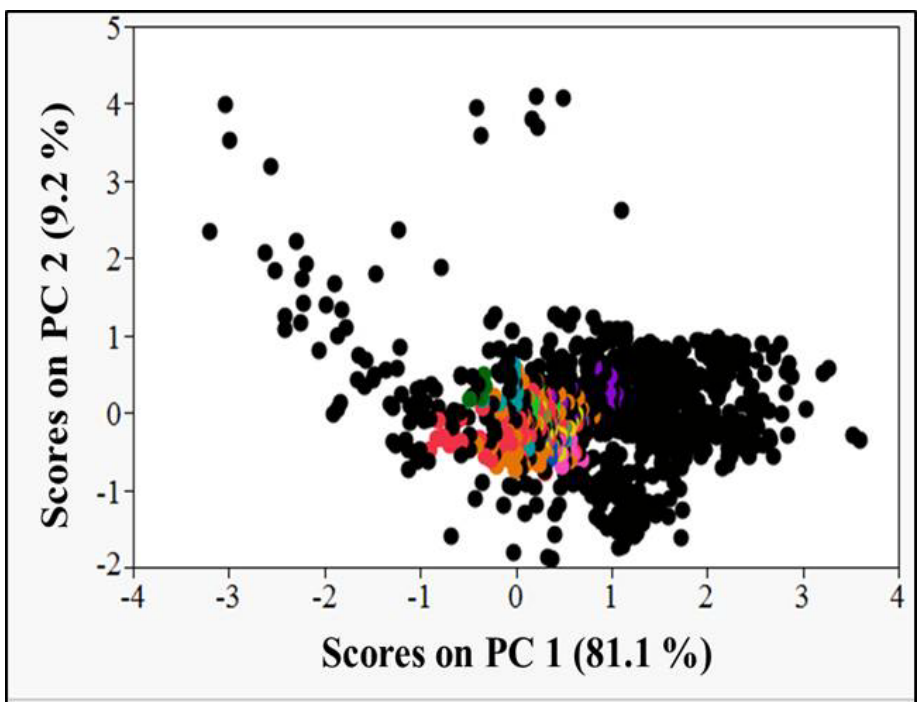

Figure 82: PCA of domestic and foreign cotton analyzed by LIBS analysis. US $=$ black, China $=$ red,

Mexico $=$ orange, Brazil $=$ yellow, Guadalupe $=$ dark green, India $=$ light green, Senegal $=$ blue,

Pakistan $=$ teal, Tanzania $=$ purple and Zimbabwe $=$ pink.

\subsection{LA-ICP-OES Results}

Interestingly, when all domestic and foreign cotton data obtained using LA-ICP-OES are compared by PCA, the resulting score plot depicts the non U.S. samples falling in between the two U.S. regions. This reiterates the importance of determining the number of groups (regions) prior to implementing a classification scheme.

Table 60: LDA results for domestic and foreign cotton analysis by LA-ICP-OES

\begin{tabular}{|l|c|c|c|}
\hline Classifier & Non U.S. & S.E. U.S. & S.W. U.S. \\
\hline Non U.S. & $86 \%$ & $14 \%$ & - \\
\hline S.E. U.S. & $2 \%$ & $97 \%$ & $1 \%$ \\
\hline S.W. U.S. & $11 \%$ & $10 \%$ & $79 \%$ \\
\hline
\end{tabular}

If we only consider misclassification in the LDA that are between U.S. and non U.S. samples we see $\sim 14 \%$ misclassification. Considering the size of the sample populations this is relatively good, an improvement over LIBS LDA of all the cotton. Using the LA-ICP-OES domestic LDA 
performance as optimal capabilities, additional foreign samples an improvement to $\sim 10 \%$ would be expected.

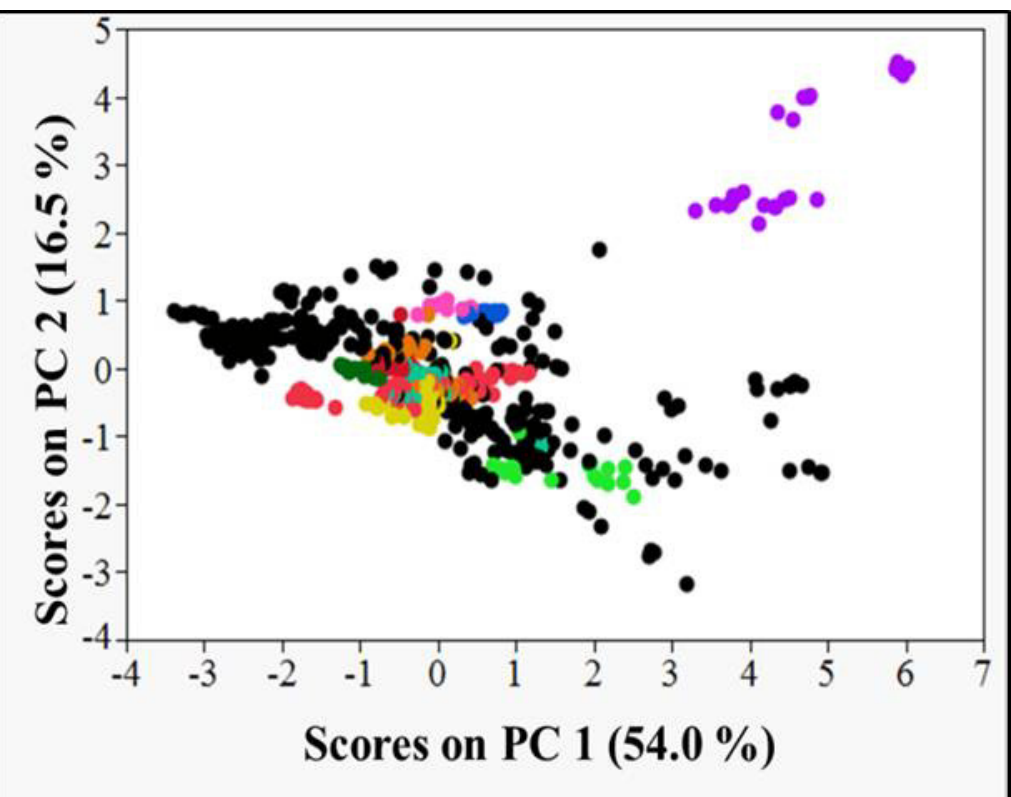

Figure 83: PCA of domestic and foreign cotton analyzed by LA-ICP-OES. US = black, China = red,

$$
\begin{gathered}
\text { Mexico }=\text { orange }, \text { Brazil }=\text { yellow }, \text { Guadalupe }=\text { dark green, } \text { India }=\text { light green, } \text { Senegal }=\text { blue }, \\
\text { Pakistan }=\text { teal, Tanzania }=\text { purple and Zimbabwe }=\text { pink }
\end{gathered}
$$

\subsection{LA-ICP-MS Results}

Using PCA, LA-ICP-MS is able to visualize similar trends in the data as presented using LA-ICPOES. Although the overlap appears to be less than that observed in LA-ICP-OES, similar LDA performance is observed. Misclassification of both U.S. and non U.S. origin occur at $\sim 15 \%$, with an expected improvement to $\sim 10 \%$ when additional samples and groups are included in the predictive modeling.

The similarity in LDA performance for both domestic and foreign comparisons by either ICP technique insinuates that both instrumental approaches, regardless of analytical figures of merit, yield similar modeling capabilities. 


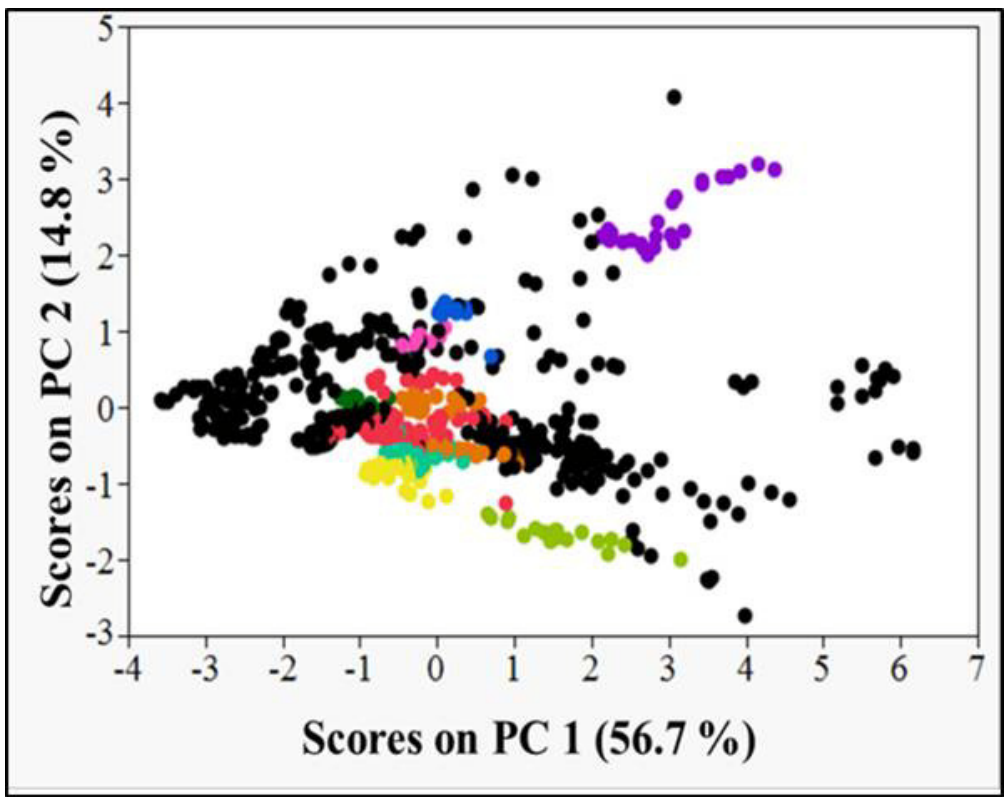

Figure 84: PCA of domestic and foreign cotton analyzed by LA-ICP-MS. US = black, China = red,

Mexico $=$ orange, Brazil $=$ yellow, Guadalupe $=$ dark green, India $=$ light green, Senegal $=$ blue,

Pakistan $=$ teal, Tanzania $=$ purple and Zimbabwe $=$ pink.

Table 61: LDA results for domestic and foreign cotton analysis by LA-ICP-MS

\begin{tabular}{|l|c|c|c|}
\hline Classifier & Non U.S. & S.E. U.S. & S.W. U.S. \\
\hline Non U.S. & $83 \%$ & $17 \%$ & - \\
\hline S.E. U.S. & $<1 \%$ & $>99 \%$ & - \\
\hline S.W. U.S. & $14 \%$ & $9 \%$ & $77 \%$ \\
\hline
\end{tabular}

\subsubsection{LDA: Country Comparisons}

The use of geographic provenancing for cotton classification and authentication at U.S. ports can also be used to particular comparisons. Tariffs are assigned not only on the basis of the quality of the good, but also the origin of the good. The U.S. has several trade agreements in place that result in lower or preferential tariff assignments for particular countries. The most well-known of the free trade agreements (FTAs) in particular for cotton commodities are the Central American Free Trade Agreement (CAFTA) and the North American Free Trade Agreement (NAFTA) ${ }^{183,184}$. 
This provides almost open-access to U.S. markets for countries under the umbrella FTA. In addition, the U.S. also benefits when exporting goods to these countries.

The presence of FTAs poses a threat to effectively upholding other tariffs at U.S. ports. This is especially evident for the importation of cotton commodities. To counter the difference in tariffs that pertains to the country exporting the good, cotton exporters use what is now referred to as the transshipment method ${ }^{185}$. A transshipment approach to importing cotton involves the importation of cotton in the U.S. by means of a second country. The middle country is then listed as the country of origin for the commodity. A prominent example of this is China importing cotton to the U.S. through Mexico. Mexico has several FTAs with the U.S. so Chinese cotton is able to, with solely paper documentation, gain access to U.S. markets by claiming Mexico origin. Because the U.S. is in a constant state of trade embargos and extremely high tariffs for Chinese good importation, resulting in a loss of revenue or a flux of unauthorized product into U.S. markets.

Geographic provenancing was also evaluated for this particular comparison of Chinese and Mexican grown cotton. Because this technique was selected because it performed better than LIBS and comparable to LA-ICP-MS. LA-ICP-OES offers similar performance with other attractive features that suggest this technique is more appropriate for this application.

To combat potential cotton transshipments, the elemental profiles of cotton that originated in China, Mexico and the U.S. were compared using discriminant analysis. The samples were limited to the number of available replicated of China and/or Mexico. Because of the limited sample pool in general, the samples were not split into the three LDA tiers (train, test and validate). Rather, the model was built using all samples from each classifier. The testing phase of the model consisted of removing five samples and re-determining the LDF. This was conducted at random until each sample had been left out at least once. 


\subsection{LA-ICP-OES Results}

Overall success rates are presented in the LDA summary tables. When comparing all countries involved in the transshipment process model performance exceeds that obtained when all foreign samples were used. This suggests that the separation of foreign cotton (with more samples) into smaller regions will improve the ability to classify on the basis of geographic information

Table 62: LDA of all countries involved in transshipment

\begin{tabular}{|l|c|c|c|c|}
\hline Classifier & China & Mexico & S.E. U.S. & S.W. U.S. \\
\hline China & $89 \%$ & - & $11 \%$ & - \\
\hline Mexico & - & $98 \%$ & - & $2 \%$ \\
\hline S.E. U.S. & $2 \%$ & - & $98 \%$ & - \\
\hline S.W. U.S. & $9 \%$ & $7 \%$ & - & $84 \%$ \\
\hline
\end{tabular}

Even when the U.S. is considered as a single region, assignments can be made. However, more classification of Chinese cotton occurs.

Table 63: LDA of all countries involved in transshipment - 2

\begin{tabular}{|l|c|c|c|}
\hline Classifier & China & Mexico & U.S. \\
\hline China & $71 \%$ & - & $19 \%$ \\
\hline Mexico & - & $98 \%$ & $2 \%$ \\
\hline U.S. & $20 \%$ & $3 \%$ & $77 \%$ \\
\hline
\end{tabular}

The essential comparison to combat this potential misuse of a FTA is to determine if Chinese and Mexican grown cotton can be separate. On the basis of the samples available to the study, the regions could be completely separated with no potential misclassification.

Table 64: LDA comparing Chinese and Mexican cotton classification

\begin{tabular}{|l|c|c|}
\hline Classifier & China & Mexico \\
\hline China & $100 \%$ & - \\
\hline Mexico & - & $100 \%$ \\
\hline
\end{tabular}


This enhances the significance of this research because not only have real world large scale applications been identified, but the ability to perform geographic provenancing on a small scale can be just as useful.

An additional LDA comparison of China, Mexico and the U.S was generated using all elemental analytes (Al, Ba, Fe, $\mathrm{Mg}, \mathrm{Mn}$ and $\mathrm{Sr}$ ) as well as $\delta^{18} \mathrm{O}$ information. The oxygen information was selected because of the predictive abilities of the analyte when utilizing regional information. The small error in the regression model built in chapter 8 emphasizes the correlation of the unprocessed cotton $\delta^{18} \mathrm{O}$ association to geographic region and therefore was expected to improve the classifications over including $\delta^{2} \mathrm{H}$ or both $\delta^{18} \mathrm{O}$ and $\delta^{2} \mathrm{H}$.

Because the U.S. samples analyzed by IRMS outnumbered the China and Mexico samples, actual sample inclusions were selected at random, and the model was generated over several iterations so that each U.S. sample was included in the model assessment at least once. A similar process was used for the Mexican cotton data so as to build a model that utilized equal numbers of samples associated with each classifier (country). In all iterations (17 were required) no misclassification for Mexico, China or U.S. unprocessed cotton was observed. The improvements in group assignment following the addition of $\delta^{18} \mathrm{O}$ information suggests that using a combination of the two types of analytes for geographic provenancing of an unprocessed cotton matrix has the potential to be more appropriate for this particular application.

\subsubsection{Establishing Geographic Trends Using Elemental and Stable Isotope Information}

Elemental and stable isotope information has been useful to accomplishing geographic provenancing of unprocessed cotton separately. However, additional information could be 
obtained when these two approaches are combined. Also, correlations could also exist and relationships between these two types of regional information could be established.

It has been shown that the presence of salts in water have the potential to influence fractionation in water. The presence of salts in solution effect isotopic properties of water ${ }^{155}$. This has been hypothesized to be related to the interaction between the hydration sphere of the cation and the water molecule. The degree of fractionation is related to the strength of the cation- $\mathrm{H}_{2} \mathrm{O}$ bonds in comparison to interactions between water molecules. The weaker the interaction with the cation the more likely a negative isotope fractionation will occur and vice versa ${ }^{155}$. The influence of some cations that have been used in this research $(\mathrm{Al}, \mathrm{Fe}, \mathrm{Mg})$ are presented in figure 85 .

This trend was evaluated to determine if this relationship could be sustained after uptake by the plant. The only element that should correlation to the $\delta^{18} \mathrm{O}$ was barium, figure 86 . The ability to visualize the trend with Ba suggests that remnants of this relationship persist beyond biochemical processes in the plant.

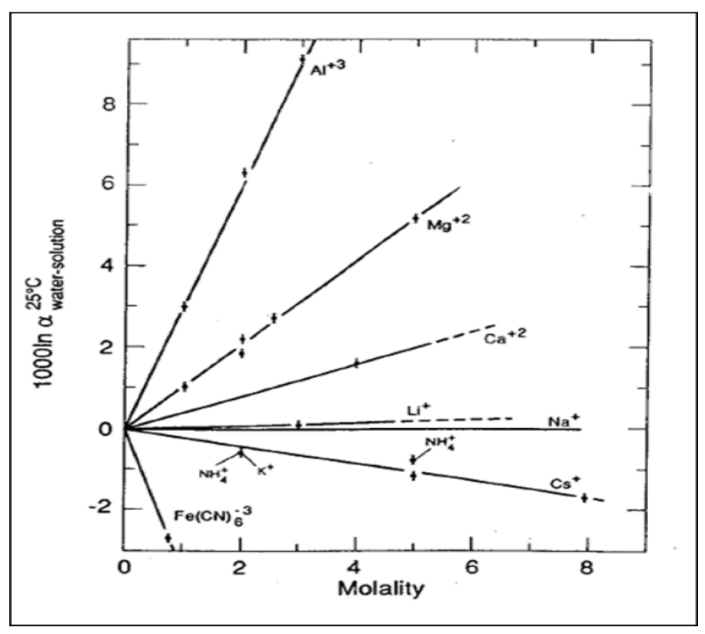

Figure 85: Influence of cations on oxygen isotope fractionation in water where the rate of the reaction is equivalent to $\alpha={ }^{18} \mathrm{O} /{ }^{16} \mathrm{O}(\mathrm{y} \text {-axis })^{155}$. 


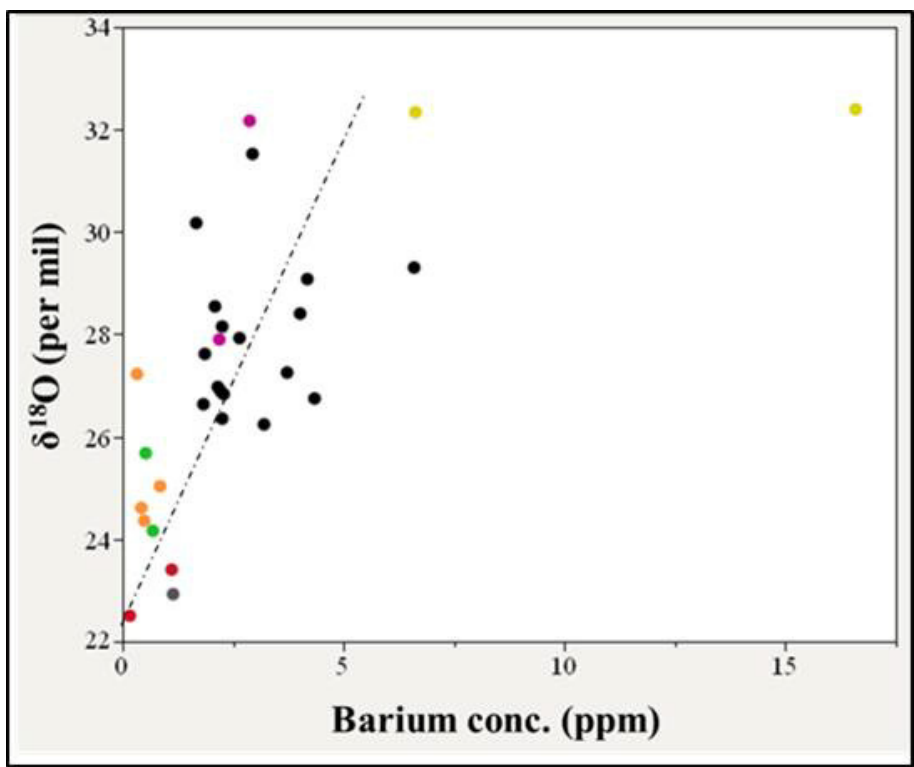

Figure 86: Correlation of $[\mathrm{Ba}]$ to $\delta^{18} \mathrm{O}$ in cotton, black $=$ U.S., red $=$ China, orange $=$ Mexico, yellow $=$ Tanzania, green $=$ Pakistan, pink $=$ India and gray $=$ Brazil

\subsubsection{Comparison Utilizing PCA}

Visualization of potential groups was accomplished using PCA to compare both elemental and stable isotopic information. Aluminum was not included in the PCA because its contribution to both eigenvectors was less than 0.05 . Separation between domestic and foreign cotton can be obtained by using an imaginary boundary at PC $2=0$. This also results in closer approximate distances between samples from the same country. Utilizing both elemental and isotopic information, while not completely independent as demonstrated in figure 86 , yields enhancement in the separation that can both be attributed to geographic region. When used together the geographic variation is amplified. This suggests that further exploration of the relationship should be explored to strengthen model performance beyond that which can be obtained with elemental or even stable isotope data alone. 


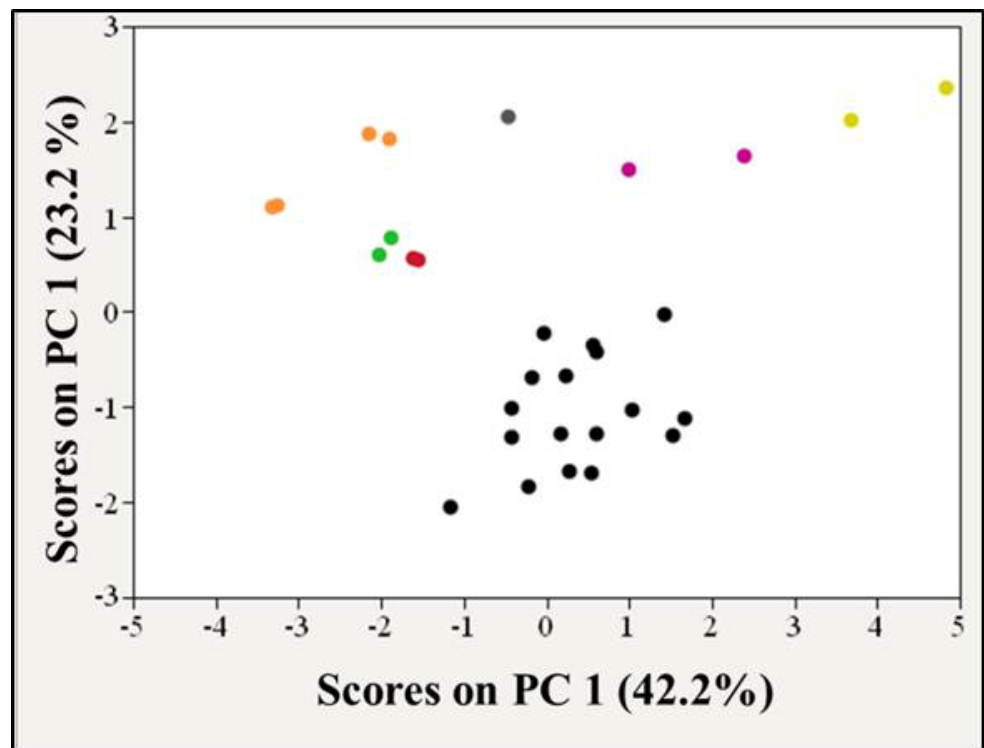

Figure 87: PCA of elemental and stable isotope results to determine additional trends pertaining to geographic region; black $=$ U.S., red $=$ China, orange $=$ Mexico, yellow $=$ Tanzania, green $=$ Pakistan, pink $=$ India and gray $=$ Brazil.

In addition the overall purpose of the analysis for the intended matrix should be considered when determining the type of analyte - elemental or stable isotope ratio - is appropriate. Here the matrix is completely unprocessed and therefore both the elemental and stable isotope ratio information depends on the region. However, if the matrix analyzed corresponds to any cotton following any stage in the processing of the fibers for the manufacture of textile related goods the selection of analyte is more critical. Elements present in the cotton have the potential to be water soluble and thus can be washed away during processing or, additional elements or amounts may be introduced, resulting in elemental profiles that do not correlate to a geographic region.

However, it has been shown that the use of stable isotope ratios can be used differentiate cotton textiles on the basis of geographic origin ${ }^{161}$. The ability to do so implies that any change in stable isotope ratio occurs in proportion and therefore the underlying differences associated with geographic regional information remain. Therefore the state of the cotton matrix plays a role in the analytical approach that is appropriate for making geographic associations. 


\subsubsection{Regression Analysis}

As a method to assess the stability of the element profiles observed, regression modeling was used. Each model was built to predict the concentration of one element on the basis of the concentrations of other elements. An example of the correlation of predicted vs. actual concentration is shown in figure 88. All domestic cotton samples harvested from all years available were included in all of the regression models.

The element concentrations present in cotton demonstrates stability in modeling this data. This suggests that even as the elemental profile evolves over time it will do so in a predictive, scaled manner for all elements used in this research. This also suggests that differences currently observed that are used to separate groups will remain over time since several crop years were used to generate this model.

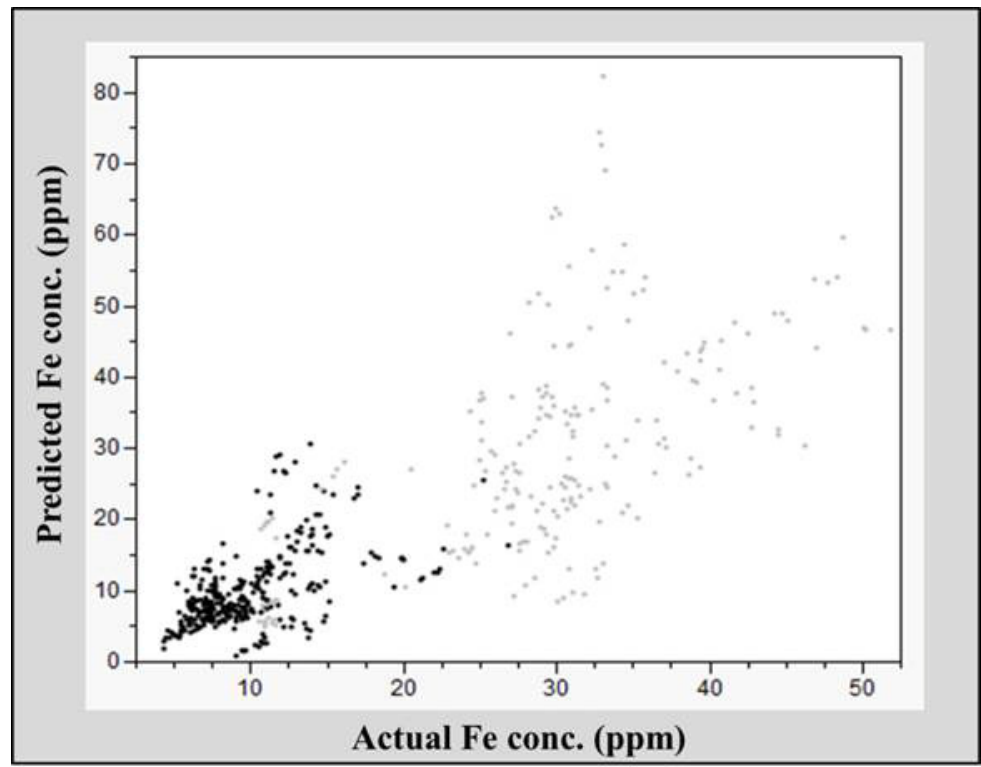

Figure 88: Regression model results for [Fe] determination. 


\subsection{Discussion and Conclusions}

Elemental analysis and stable isotope information of unprocessed cotton could be used as separate techniques or in combination in order to separate unprocessed cotton sources according to geographic attributes. When possible, modeling of this data was performed and resulted in successful attempts to predict the geographic assignment of individual cotton samples. The extensive domestic cotton sample set was used to model variation that could possibly be encountered in other cotton producing regions throughout the world. The extent to which correct assignment of southeast and southwest cotton to the actual originating region could be accomplished using elemental analysis techniques were used to infer model performance that would result from foreign vs. U.S. comparisons as well as other country comparisons of this nature.

The significant amount of information available regarding the domestic cotton samples allowed for an in-depth evaluation of the inherent heterogeneity of the matrix, as well as an assessment of the influence of environmental contributions on the resulting elemental profile of unprocessed cotton to be made. The results of these studies concluded that, using the element menu selected in this research, the elemental profile of the cotton reflects the geographic region from which the cotton originated without additional contributions from outside factors. In addition, because the concentration of an element could be predicted using the concentrations of other elements analyzed confirms that the U.S. geographic regions, in terms of the elemental profile of cotton, will remain over time. Changes that do occur will do so in a scaled manner. To a point this has already been demonstrated since the predictions of element concentration were made using all domestic cotton across all available harvest years. Using domestic cotton as an exemplar to predict characteristics of foreign grown cotton it is expected that this concept of elemental stability also applies. 
The ability to detect differences in the cotton sources using elemental analysis could be completed with all elemental techniques considered within the scope of this dissertation. However, because of the similarity in capability to LA-ICP-MS, and its ability to outperform LIBS, LA-ICP-OES was selected as the most appropriate method to conduct elemental analysis. This technique is less expensive to acquire and maintain than LA-ICP-MS, however is able to offer analogous performance for this application. In addition, this technique was sensitive to temporal variation, which may be useful in future comparisons.

Differences between U.S. and foreign grown cotton could be established when either or both analytical techniques were employed. Improvements, however, in elemental analysis model success rates were observed when foreign data was broken down into smaller regions, China and Mexico for example. This suggests that with additional samples, elemental and even isotopic ratio information can be used to authenticate cotton growing region. In addition the ability to correctly distinguish cotton from China and Mexico is significant in and of itself because current real world implications of this ability.

While enhanced separation of different geographic regions was observed when both elemental and stable isotope information regarding the overlapping sample set were compared, the selection of both types of analytes or solely elemental or stable isotope analysis should be determined on the basis of the stage in processing of the cotton. It is possible for elemental constituents to change over the course of cotton processing and to do so without accordance to a geographic pattern, whereas previous work demonstrates that variation in the stable isotopes present even in cotton textiles can be used for geographic comparisions ${ }^{161}$. 


\subsection{RESEARCH CONCLUSIONS}

The research presented in the previous chapters of this dissertation address the ability to implement an independent means by which geographic location or region can be identified to authenticate or refute paper documentation provided at U.S. ports for the importation of unprocessed cotton. Geographic provenancing can be accomplished using either or both elemental and stable isotopic information present in the cotton. The analytes have been demonstrated as possessing regional information and provide a method for distinguishing various sources of cotton. The ability to visualize and eventually generate models to predict the geographic source was fulfilled using LDA. Although LDA has been reported in the literature, this research developed a three-tier approach that applied several iterations of the data to produce a model of good quality to withstand temporal variation. This stresses the competence of the approach developed in the dissertation.

As a result of the small scale of the stable isotope study, most conclusions offered here focus on the comparisons and achievements of the research in respect to elemental analysis. The results obtained thus far demonstrate that stable isotopes offer complementary information to elemental analysis capable of enhancing the capacity when used together for geographic provenancing. Further exploration is warranted so as to thoroughly evaluate the information that can be obtained from this analysis. The current study is considered a proof of concept for this particular application.

A comparison of the performance of atomic emission spectroscopy techniques were presented as an alternative to inorganic MS for elemental analysis. The coupling of laser ablation to ICP-OES resulted in superior performance to that which could be obtained using LIBS, when either the analytical figures of merit or multivariate modeling capabilities are considered. From the similarity in analytical performance of the ICP techniques it can be concluded that LA-ICP-OES 
is an appropriate, if not the most appropriate elemental technique for this application. The lower cost and less time for maintenance as well as the fewer parts that require maintenance is attractive to laboratories with a tight budget. While LA-ICP-MS is beyond the reach of most forensic laboratories, LA-ICP-OES offers a more economical alternative. In addition this technique has demonstrated similar performance to that which has been reported for LA-ICP-MS for the same set of glass samples. The studies presented within the scope of this dissertation address the feasibility of implementing LA-ICP-OES for forensic applications.

Although the use of this approach as a solid sampling technique for analytical applications is not commonly presented, research conducted within this dissertation strives to advance the quality of measurement that can be obtained by evaluating the plasma conditions in ICP-OES. This has resulted in a simpler instrumental setup without the sacrifice of sensitivity, with sub ppm LODs being reported here for most analytes utilized in the analysis of glass and cotton. This is beneficial to both the analyst as well as the laboratory, resulting in less time consuming instrumental setup and thus more time dedicated to sample analysis.

While LA-ICP-OES is recommended for this application, LIBS does not perform poorly. Research conducted within Part I of this dissertation address the need for standardization amongst LIBS users so as to yield more uniform results between laboratories, independent of the in-house or commercial LIBS system built. Using measurements of the plasma conditions and the ablation processes, advancements towards universal LIBS parameters were presented for several matrices of different physical properties. In addition these parameters were determined to be approximately uniform regardless of laser irradiation wavelength or method of emission collection when $532 \mathrm{~nm}$ and $1064 \mathrm{~nm}$ irradiation wavelengths are considered. The studies lay provide foundation for the potential adoption of universal parameters for analytical LIBS analysis. With the implementation of these standardized parameters into commercial LIBS 
systems it is expected that improvements in the utility of LIBS to result in comparable performance in comparison to ICP techniques for the sample matrices studies, will be realized.

The importance of this research for the forensic community includes offering a solution to a current problem using techniques that are within research of forensic laboratories. In addition, the versatility of LA-ICP-OES for other forensic analyses is presented, enhancing the appeal of this elemental analysis technique. Simplification of elemental analysis techniques are also presented in order to reduce the complexity of the analysis, transferring the techniques from the research realm to the operational forensic analysis laboratory. 


\section{Reference List}

1. Cook, J. G. Handbook of Textile Fibres: Natural Fibres; Merrow: Great Britian, 1984.

2. Baldwin, D. Testimony at the House Committee on Small Business Subcommittee on Rural Development, Entrepreneurship and Trade. 2009.

3. Cahoon, E. M.; Almirall, J. R. Wavelength dependence on the forensic analysis of glass by nanosecond $266 \mathrm{~nm}$ and $1064 \mathrm{~nm}$ laser induced breakdown spectroscopy. Appl. Opt. 2010, 49 (13), C49-C57.

4. Boss, C. B.; Fredeen, K. J. Concepts, Instrumentation and Techniques in Inductively Coupled Plasma Optical Emission Spectrometry; 2nd ed.; PerkinElmer Corporation: Norwalk, CT, 1997.

5. Inductively Coupled Plasma in Analytical Atomic Spectrometry; 2 ed.; VCH Publishers: New York, 1992.

6. Thompson, M.; Chenery, S.; Brett, L. Calibration Studies in Laser Ablation Microprobe Inductively Coupled Plasma Atomic Emission Spectrometry. J. Anal. At. Spectrom. 1998, 4, 11-16.

7. Brech, F.; Cross, L. Optical microemission stimulated by a ruby laser. Appl. Spectrosc. 1962, 16, 59 .

8. Cremers, D. A.; Radziemski, L. J. Handbook of Laser-Induced Breakdown Spectroscopy; John Wiley and Sons Ltd: West Sussex, England, 2006.

9. Laser Induced Breakdown Spectroscopy: Fundamentals and Applications; Cambridge University Press: New York, 2006.

10. Laser-Induced Breakdown Spectroscopy; Elsevier: New York, 2007.

11. Kaminskii, A. A. Laser Crystals: Their Physics and Properties; 2nd ed.; SpringerVerlag: New York, 1975; Vol. 14.

12. Silfvast, W. T. Laser Fundamentals; Cambridge University Press: New York, 1996.

13. De Giacomo, A.; Gaudiuso, R.; Dell'Aglio, M.; Santagata, A. The role of continuum radiation in laser induced plasma spectroscopy. Spectrochim. Acta Part B 2010, $65,385-394$.

14. Cristoforetti, G.; Legnaioli, S.; Pardini, L.; Palleschi, V.; Tognoni, E. Spectroscopic and shadowgraphic analysis of laser induced plasmas in the orthogonal double pulse pre-ablation configuration. Spectrochim. Acta Part B 2006, 61, 340-350.

15. Kramida, A.; Ralechenko, Yu.; Reader, J. NIST Atomic Spectra database (ver. 5.0). available at http://physic.nist.gove/asd. 2012. Gaithersburg, MD, National Institute of Standards and Technology. 
16. Martin, M. Z.; Labbe, N.; Andre, N.; Harris, R.; Ebinger, M.; Wullschleger, S. D.; Vass, A. A. High resolution applications of laser-induced breakdown spectroscopy for environmental and forensic applications. Spectrochim. Acta Part B 2007, 62 (12), $1426-1432$.

17. Jantzi, S. C.; Almirall, J. R. Characterization and forensic analysis of soil samples using laser-induced breakdown spectroscopy (LIBS). Anal. Bioanal. Chem. 2011, 400, $3341-3351$.

18. Hybl, J. D.; Lithgow, G. A.; Buckley, S. G. Laser-Induced Breakdown Spectroscopy Detection and Classification of Biological Aerosols. Appl. Spectrosc. 2003, 57 (10), 1207-1215.

19. Yaroshchyk, P.; Morrison, R. J. S.; Body, D.; Chadwick, B. L. Quantitative determination of wear metals in engine oils using LIBS: The use of paper substrates and a comparison between single-and double-pulse LIBS. Spectrochim. Acta Part B 2005, 60, 1482-1485.

20. Niemax, K. Laser ablation - reflections on a very complex technique for solid sampling. Fresenius. J. Anal. Chem. 2001, 370, 332-340.

21. Sirven, J.-B.; Mauchien, P.; Salle, B. Analytical optimization of some parameters of a Laser-Induced Breakdown Spectroscopy Experiment. Spectrochim. Acta Part B 2008, 63, 1077-1084.

22. Brygo, F.; Dutouquet, C.; Le Guern, F.; Oltra, R. Laser fluence repetition rate nad pulse duration effects on paint ablation. Appl. Surf. Sci. 2006, 252, 2131-2138.

23. Mao, S. S.; Zeng, X.; Mao, X.; Russo, R. E. Laser-induced breakdown spectroscopy: flat surface vs. cavity structures. J. Anal. At. Spectrom. 2004, 19, 495-498.

24. Mao, X. L.; Ciocan, A. C.; Borisov, O. V.; Russo, R. E. Laser ablation processes investigated using inductively coupled plasma-atomic emission spectroscopy (ICP-AES). Appl. Surf. Sci. 1998, 127, 262-268.

25. Shannon, M. A.; Mao, X.; Fernandez, A.; Chan, W.-T.; Russo, R. E. Laser Ablation Mass Removal versus Incident Power Density during Solid Sampling for Inductively Coupled Plasma Atomic Emission Spectroscopy. Anal. Chem. 1995, 67, 45224529 .

26. Horn, I.; Guillong, M.; Gunther, D. Wavelength dependent ablation rates for metals and silicate glasses using homogenized laser beam profiles - implications for LAICP-MS. Appl. Surf. Sci. 2001, 182, 91-102.

27. Schenk, E. R.; Almirall, J. R. Elemental analysis of cotton by laser-induced breakdown spectroscopy. Appl. Opt. 2010, 49 (13), C153-C160.

28. Wang, Q.; Jander, P.; Fricke-Bergemann, C.; Noll, R. Comparison of 1064 nm and 266 $\mathrm{nm}$ excitation of laser-induced plasmas for several types of plastics and one explosive. Spectrochim. Acta Part B 2008, 63, 1011-1015. 
29. Salle, B.; Chaleard, C.; Lacour, J. L.; Mauchien, P.; Nouvellon, C.; Semerok, A. Laser ablation efficiency of metal samples with UV laser nanosecond pulses. Appl. Surf. Sci. 1999, 138-139, 302-305.

30. Semerok, A.; Chaleard, C.; Detalle, V.; Lacour, J.-L.; Mauchien, P.; Meynadier, P.; Nouvellon, C.; Salle, B.; Palianov, P.; Perdrix, M.; Petite, G. Experimental investigations of laser ablation efficiency of pure metals with femto, pico and nanosecond pulses. Appl. Surf. Sci. 1999, 138, 311-314.

31. Mateo, M. P.; Cabalin, L. M.; Baema, J. M.; Laserna, J. J. Surface interaction and chemical imaging in plasma spectrometry induced with a line-focused laser beam. Spectrochim. Acta Part B 2002, 57, 601-608.

32. Hahn, D. W.; Omenetto, N. Laser-Induced Breakdown Spectroscopy (LIBS), Part 1:

Review of Basic Diagnostics and Plasma-Particle Interactions: Still-Challenging Issues Within the Analytical Plasma Community. Appl. Spectrosc. 2010, 64, 335A-366A.

33. Nolte, J. ICP Emission Spectrometry: A Practical Guide; Wiley-VCH: Germany, 2003.

34. Lazar, A. C.; Farnsmorth, P. B. Matrix Effect Studies in the Inductively Coupled Plasma with Monodisperse Droplets. Part II: The Influence of Matrix on Spatially Integrated Ion Density. Appl. Spectrosc. 1999, 53 (4), 465-470.

35. G.C.-Y.Chan; W.-T.Chan; X.Mao Investigation of matrix effects in inductively coupled plasma-atomic emission spectroscopy using laser ablation and solution nebulization - effect of second ionization potential. Spectrochim. Acta Part B 2001, 56, 77-92.

36. Lotito, G.; Nelis, T.; Guillot, P.; Gunther, D. Characterization of argon metastable species as function of time, space and current of a pulsed dc glow discharge.

Spectrochim. Acta Part B 2011, 66, 619-626.

37. Payling, R.; Larkins, P. Optical Emission Lines of the Elements; John Wiley and Sons: 2000.

38. Chan, G. C.-Y.; Hieftje, G. M. Investigation of charge transfer with non-argon gaseous species in mixed-gas inductively coupled plasma-atomic emission spectrometry. Spectrochim. Acta Part B 2007, 62, 196-210.

39. Farnsworth, P. B.; Omenetto, N. The kinetics of charge exchange between argon and magnesium in the inductively coupled plasma. Spectrochim. Acta Part B 1993, $46,843-850$.

40. Burton, L. L.; Blades, M. W. Charge transfer between Ar and Mg in an inductively coupled plasma. Spectrochim. Acta Part B 1991, 46, 819-830.

41. Morishige, Y.; Kimura, A. Ionization Interference in Inductively Coupled Plasma-Optical Emission Spectroscopy. Industr. Mat. 2011. 
42. Chan, G. C.-Y.; Hieftje, G. M. In-situ determination of cross-over point for overcoming plasma-related matrix effects in inductively coupled plasma-atomic emisssion spectrometry. Spectrochim. Acta Part B 2008, 63, 355-366.

43. Blades, M. W.; Horlick, G. Interference from easily ionizable element matrices in inductively coupled plasma emission spectrometry - a spatial study. Spectrochim. Acta Part B 1981, 36 (9), 881-900.

44. Dubuisson, C.; Poussel, E.; Todoli, J. L.; Mermet, J. M. Effect of sodium during the aerosol transport and filtering in inductively coupled plasma atomic emission spectrometry. Spectrochim. Acta Part B 1998, 53, 593-600.

45. Thompson, M.; Ramsey, M. H. Matrix Effects Due to Calcium in Inductively Coupled Plasma Atomic-emission Spectrometry: Their Nature, Source and Remedy. Analyst 1985, 110, 1413-1422.

46. Chan, G. C.-Y. Investigation of plasma-related matrix effects in inductively coupled plasma-atomic emission spectrometry caused by matrices with low second ionization potentials - identification of the secondary factor. Spectrochim. Acta Part B 2006, 61, 642-659.

47. Velitchkova, N.; Velichkov, S.; Daskalova, N. Inductively coupled plasma atomic emission spectrometry - Optimization of the operating conditions in the determination of trace of elements in line-rich emission matrices. Spectrochim. Acta Part B 2007, 62, 386-402.

48. Brenner, I. B.; Zischka, M.; Maichin, B.; Knapp, G. Ca and Na interferences in an axially viewed ICP using low and high aerosol loadings. J. Anal. At. Spectrom. 1998, 13, $1257-1264$.

49. O'Hanlon, K.; Ebdon, L.; Foulkes, M. Effect of Easily Ionisable Elements on the Mass Transport Efficiency of Solutions and Slurries Used in Plasma Emission Spectrometry. J. Anal. At. Spectrom. 1997, 12, 329-331.

50. Ramsey, M. H.; Thompson, M. A Predictive model of Plasma Matrix effects in Inductively Coupled Plasma Atomic Emission Spectrometry. J. Anal. At. Spectrom. 1986, 1, 185-193.

51. Dubuisson, C.; Poussel, E.; Todoli, J. L.; Mermet, J. M. Effect of sodium during the aerosol transport and filtering in inductively coupled plasma atomic emission spectrometry. Spectrochim. Acta Part B 1998, 53, 593-600.

52. Serapinas, P.; Ezerinskis, Z.; Juzikiene, V. Effect of matrices with low second ionization potentials on analyte signals in inductively coupled plasma mass spectrometry. $J$. Anal. At. Spectrom. 2011, 26, 1997-2005.

53. Skoog, D. A.; West, D. A.; Holler, F. J.; Crouch, S. R. Fundamentals of Analytical Chemistry; 8th ed.; Thomson Learning: 2004. 
54. Skoog, D. A.; Holler, F. J.; Crouch, S. R. Principles of Instrumental Analysis; 6th ed.; Thomson Learning: 2006.

55. Brenner, I. B.; Zander, A. T. Axially an radially viewed inductively coupled plasmas - a critical review. Spectrochim. Acta Part B 2000, 55, 1195-1240.

56. Dual View ICP: the best of both worlds. ICP Optical Emission Technical Note . 2008. PerkinElmer.

57. Taylor, H. E. Inductively Coupled Plasma-Mass Spectrometry; Academic Press: New York, 2001.

58. Aragon, C.; Aguilera, J. A. Characterization of laser induced plasmas by optical emission spectroscopy: A review of experiments and methods. Spectrochim. Acta Part B 2008, 63, 893-916.

59. Jankowski, K.; Dreger, M. Study of an effect of easily ionizable elements on the excitation of 35 elements in an Ar-MIP system coupled with solution nebulization. J. Anal. At. Spectrom. 2000, 15, 269-276.

60. Tripkovic, M. R.; Holclajtner-Antunovic, I. D. Study of the Matrix Effect of Easily and Non-easily Ionizable Elements in an Inductively Coupled Argon Plasma Part I. Spectroscopic Diagnostics. J. Anal. At. Spectrom. 1993, 8, 349-357.

61. Holclajtner-Antunovic, I. D.; Tripkovic, M. R. Study of the Matrix Effect of Easily and Non-easily Ionizable Elements in Inductively Coupled Argon Plasma Part 2. Equilibrium Plasma Composition. J. Anal. At. Spectrom. 1993, 8, 359-365.

62. Chaleard, C.; Mauchien, P.; Andre, N.; Uebbing, J.; Lacour, J.-L.; Geersten, C. Correction of Matrix Effects in Quantitative Elemental Analysis With Laser Ablation Optical Emission Spectrometry. J. Anal. At. Spectrom. 1997, 12, 183188.

63. Dahai, S.; Zhanxia, Z.; Haowen, Q.; Mingxiang, C. Matrix effects due to metallic elements in inductively coupled plasma emission spectrometry. Spectrochim. Acta Part B 1988, 43, 391-403.

64. Goldwasser, A.; Mermet, J. M. Contribution of the charge-transfer process to the excitation mechanisms in inductively coupled plasma atomic emission spectrometry. Spectrochim. Acta Part B 1986, 41, 725-739.

65. Farnsworth, P. B.; Smith, B. W.; Omenetto, N. An investigation of the balance of the charge exchange between $\mathrm{Mg}$ and Ar ions in the ICP. Spectrochim. Acta Part B 1991, 46, 819-843.

66. Chan, G. C.-Y.; Hieftje, G. M. Investigation of plasma-related matrix effects in inductively coupled plasma-atomic emission spectrometry cause by matrices with low second ionization potentials-identification of the secondary factor. Spectrochim. Acta Part B 2006, 61, 642-659. 
67. Dennaud, J.; Howes, E.; Poussel, E.; Mermet, J. M. Study of ionic-to-atomic line intensity ratios for two axial viewing-based inductively coupled plasma atomic emission spectrometry. Spectrochim. Acta Part B 2001, 56, 101-112.

68. Mao, X.; Chan, W. T.; Russo, R. E. Influence of Sample Surface Condition on Chemical Analysis Using Laser Ablation Inductively Coupled Plasma Atomic Emission Spectroscopy. Appl. Spectrosc. 1997, 51 (7), 1047-1054.

69. Lam, R.; Salin, E. D. Analysis of pharmaceutical tablets by laser ablation inductively coupled plasma atomic emission spectrometry and mass spectrometry (LA-ICPAES and LA-ICP-MS). J. Anal. At. Spectrom. 2004, 19, 938-940.

70. Musil, P.; Otruba, V.; Kanicky, V.; Mermet, J. M. Determination of elements in agricultural soils using IR laser ablation inductively coupled plasma atomic emission spectrometry. Spectrochim. Acta Part B 2000, 55 (11), 1747-1758.

71. Guillong, M.; Gunther, D. Effect of particle size distribution on ICP-induced elemental fractionation in laser ablation-inductively coupled plasma-mass spectrometry. $J$. Anal. At. Spectrom. 2002, 17, 831-837.

72. Horn, I.; Gunther, D. The influence of ablation carrier gases Ar, He and $\mathrm{Ne}$ on the particle size distribution and transport efficiencies of laser ablation-induced aerosols: implications for LA-ICP-MS. Appl. Surf. Sci. 2003, 207, 144-157.

73. Miclea, M.; Garcia, C. C.; Exius, I.; Lindner, H.; Niemax, K. Emission spectroscopic monitoring of particle composition, size and transport in laser ablation inductively coupled plasma spectrometry. Spectrochim. Acta, Part B 2006, 61, 361-367.

74. Koch, J.; Walle, M.; Schlamp, S.; Rosgen, T.; Gunther, D. Expansion phenomena of aerosols generated by laser ablation under helium and argon atmosphere. Spectrochim. Acta Part B 2008, 63, 37-41.

75. Leach, A. M.; Hieftje, G. M. Factors affecting the production of fast transient signals in single shot laser ablation inductively coupled plasma spectrometry. Appl. Spectrosc. 2002, 56, 62-69.

76. Gunther, D.; Heinrich, C. A. Enhanced sensitivity in laser ablation-ICP mass spectrometry using helium-argon mixtures as aerosol carrier. J. Anal. At. Spectrom. 1999, 14, 1363-1368.

77. Chan, G. C.-Y.; Chan, W. T.; Mao, X.; Russo, R. E. Investigation of matrix effect on dry inductively coupled plasma conditions using laser ablation sampling. Spectrochim. Acta Part B 2000, 55, 221-235.

78. Chan, G. C.-Y.; Chan, W. T.; Mao, X.; Russo, R. E. Comparison of matrix effects in inductively coupled plasma using laser ablation and solution nebulization for dry and wet plasma conditions. Spectrochim. Acta Part B 2001, 56, 1375-1386. 
79. Mermet, J. M.; Poussel, E. ICP Emission Spectrometers: 1995 Analytical Figures of Merit. Appl. Spectrosc. 1995, 49, A12-A18.

80. Tognoni, E.; Hidalgo, M.; Canals, A.; Cristoforetti, G.; Legnaioli, S.; Salvetti, A.; alleschi, V. Combination of the ionic-to-atomic line intensity ratios from two test elements for the diagnostic of plasma temperature and electron number density in Inductively Coupled Plasma Atomic Emission Spectroscopy. Spectrochim. Acta Part B 2007, 62, 435-443.

81. Mermet, J. M. Ionic to atomic line intensity ratio and residence time in inductively coupled plasma-atomic emission spectrometry. Spectrochim. Acta Part B 1989, 44 (11), 1109-1116.

82. Mao, X.; Ciocan, A. C.; Borisov, O. V.; Russo, R. E. Laser ablation processes investigated using inductively coupled plasma-atomic emission spectroscopy (ICP-AES). Appl. Surf. Sci. 1998, 127, 262-268.

83. Figg, D.; Kahr, M. S. Elemental fractionation of glass using laser ablation inductively coupled plasma mass spectrometry. Appl. Spectrosc. 1997, 51, 1185-1195.

84. Figg, D.; Cross, J. B.; Brink, C. More investigations into elemental fractionation resulting from laser ablation-inductively coupled plasma-mass spectrometry on glass samples. Appl. Surf. Sci. 1998, 129, 278-285.

85. Gonzalez, J.; Mao, X. L.; Roy, J.; Russo, R. E. Comparison of 193, 213 and $266 \mathrm{~nm}$ wavelengths on laser ablation ICP-MS. J. Anal. At. Spectrom. 2002, 60, 27-35.

86. Russo, R. E.; Mao, X.; Liu, H. C.; Gonzalez, J.; Mao, S. S. Laser ablation in analytical chemistry - a review. Talanta 2011, 57, 425-451.

87. Russo, R. E.; Mao, X. L.; Borisov, O. V.; Liu, H. C. Influence of wavelength on fractionation in laser ablation ICP-MS. J. Anal. At. Spectrom. 2000, 15, 11151120 .

88. Gonzalez, J.; Liu, C.; Wen, S.; Mao, X. L.; Russo, R. E. Glass particles produced by laser ablation for ICP-MS measurement. Talanta 2007, 73, 577-582.

89. Gonzalez, J.; Liu, C.; Wen, S.; Mao, X. L.; Russo, R. E. Metal particles produced by laser ablation for ICP-MS measurement. Talanta 2007, 73, 567-576.

90. Russo, R. E.; Mao, X. L.; Mao, S. S. The Physics of Laser Ablation in Microchemical Analysis. Anal. Chem. 2002, 74, 70A-77A.

91. Russo, R. E.; Mao, X. L.; Liu, H. C.; Gonzalez, J. Laser ablation in analytical chemistry a review. Talanta 2002, 57, 425-451.

92. Fernandez, B.; Claveries, F.; Pecheyran, C.; Donard, O. F. X. Direct analysis of solid samples by fs-LA-ICP-MS. Trends Anal. Chem. 2007, 26 (10), 951-966. 
93. Gunther, D.; Hattendorf, B. Solid sample analysis using laser ablation inductively coupled plasma mass spectrometry. Trends Anal. Chem. 2005, 24 (3), 255-264.

94. Trejos, T.; Almirall, J. R. Sampling strategies for the analysis of glass fragments by LAICP-MS Part I. Micro-heterogeneity study of glass and its application to the interpretation of forensic evidence. Talanta 2005, 67, 388-395.

95. Trejos, T.; Almirall, J. R. Sampling strategies for the analysis of glass fragments by LAICP-MS Part II: Sample size and sample shape considerations. Talanta 2005, 67, 396-401.

96. Schenk, E. R.; Almirall, J. R. Elemental analysis of glass by laser ablation inductively coupled plasma optical emission spectrometry (LA-ICP-OES_. Forensic Sci. Int. 2012, 217 (1), 222-228.

97. Bei, L.; Duffin, K. L.; Carnahan, J. W. Inductively coupled plasma chemistry examinations with visible acousto-optic tunable filter hyperspectral imaging. $J$. Anal. At. Spectrom. 2004, 19, 1-8.

98. Becker, J. S.; Tenzier, D. Studies of LA-ICP-MS on quartz glasses at different wavelengths of a Nd:YAG laser. Fresenius. J. Anal. Chem. 2001, 370 (5), $637-$ 640 .

99. Polak, A. Glass: its tradition and its makers; G.P. Putnam's Sons: New York, 1975.

100. ASTM Standard Terminology of glass and glass products. Annual Book of ASTM Standards. ASTMC, 162. 2005.

101. Jones, G. O. Glass; 2nd ed.; Methuen \& Co. Ltd.: 1971.

102. de Jong, B. H. W. S. Ullmann's Encyclopedia of Industrial Chemistry; 7th ed.; WileyVCH: 2011.

103. Phillips, C. J. Glass: Its Industrial Applications; Reinhold Publishing Corporation: New York, 1960.

104. Almirall, J. R.; Trejos, T. Advances on the Forensic Analysis of Glass Fragments with a Focus on Refractive Index and Elemental Analysis. Forensic Sci. Rev. 2006, 18 , 76-96.

105. Almirall, J. R. Elemental analysis of glass fragments. In Trace Evidence Analysis and Interpretation: Glass and Paint, B.Caddy, Ed.; Taylor and Francis: London, 2001; pp 65-81.

106. Catterick, T.; Hickman, D. A. The Quantitative analysis of glass by inductively coupled plasma atomic emission spectroscopy: A five element survey. Forensic Sci. Int. 1981, 17 (3), 253-263.

107. Buscaglia, J. A. Elemental Analysis of small glass fragments in forensic science. Anal. Chim. Acta 1994, 288 (1-2), 17-24. 
108. Montero, S.; Hobbs, A. L.; French, T. A.; Almirall, J. R. Elemental Analysis of Glass Fragments by ICP-MS as Evidence of association: Analysis of a Case. $J$. Forensic Sci. 2001, 48 (5), 1-7.

109. Latkoczy, C.; Becker, S.; Dücking, M.; Günther, D.; Hoogewerff, J. A.; Almirall, J. R.; Buscaglia, J. A.; Dobney, A.; Koons, R. D.; Montero, S.; van der Peijl, G. J. Q.; Stoecklein, W. R. S.; Trejos, T.; Watling, J. R.; Zdanowicz, V. S. Development and Evaluation of a Standard Method for the Quantitative Determination of Elements in Float Glass Samples by LA-ICP-MS. J. Forensic Sci. 2005, 50 (6), $1-15$.

110. Trejos, T.; Montero, S.; Almirall, J. R. Analysis and Comparison of glass fragments by laser ablation inductively coupled plasma mass spectrometry (LA-ICP-MS) and ICP-MS. Anal. Bioanal. Chem. 2003, 376, 1255-1264.

111. Hickman, D. A. Elemental Analysis and the Discrimination of sheet glass. Forensic Sci. Int. 1983, 23 (2), 213-223.

112. Umpierrez, S. Elemental Analysis of Glass by Laser Ablation-Inductively Coupled Plasma-Mass Spectrometry (LA-ICP-MS) Without Matrix-Matched Standards. [Master's Thesis]. 2006. Florida International University, Miami, FL.

113. Andrasko, J.; Machly, A. The discrimination between samples of window glass by combining physical and chemical techniques. J. Forensic Sci. 1978, 22, 250-257.

114. Ryland, S. G. Sheet or Container? - Forensic Glass Comparisons with an Emphasis on Source Classification. J. Forensic Sci. 1986, 31 (4), 1314-1329.

115. Dudley, R. J.; Howden, C. R.; Taylor, T. J.; Smalldon, K. W. The discrimination and classification of small fragments of window and non-window glass using energydispersive X-ray fluorescence spectrometry. X-ray Spectrom. 1980, 9, 119-130.

116. Naes, B. E.; Umpierrez, S.; Ryland, S. G.; Barnett, C.; Almirall, J. R. A comparison of laser ablation inductively coupled plasma mass spectrometry, micro X-ray fluorescence spectroscopy, and laser induced breakdown spectroscopy for the discrimination of automotive glass. Spectrochim. Acta, Part B 2008, 63 (10), 1145-1150.

117. Hicks, T.; Monard, S. F.; Goldmann, T.; Brunelle, A.; Champod, C.; Margot, P. The classification and discrimination of glass fragments using nondestructive energy dispersive X-ray $\mu$ fluorescence. Forensic Sci. Int. 2003, 137, 107-111.

118. Hickman, D. A. A Classification Scheme for glass. Forensic Sci. Int. 1981, 17 (3), 265 281.

119. Hickman, D. A. Glass Types Identified by Chemical Analysis. Forensic Sci. Int. 1987, 33 (1), 23-46. 
120. Koons, R. D.; Fiedler, C.; Rawalt, C. Classification and discrimination of sheet and container glasses by inductively coupled plasma atomic emission spectrometry and pattern recognition. J. Forensic Sci. 1988, 33, 49.

121. American Society for Testing and Materials ASTM method E 2330-04. Standard Test Method for Determination of Trace Elements in Glass Using Inductively Coupled Plasma Mass Spectrometry (ICP-MS). ASTM Annual Book of ASTM Standards 14. 2004.

122. Ohata, M.; Furuta, N. Laser Defocusing Effects on Laser Ablation Inductively Coupled Plasma-Atomic Emission Spectrometry: Different Ablation Interaction between the Laser and Low-Alloy Steel, Fe Pellets, and a Pond Sediment Pellet. Anal. Sci. 2004, 20, 701-706.

123. Goodyear, K. L.; McNeill, S.; Coles, B. J. A comparison of the geochemistry of Fe-Mn coatings on pebbles and Potamopgyrus (Hydrobia) jenkinsi shells, Allen River, SW England, UK. Appl. Geochem. 2000, 15, 725-735.

124. Gilon, N. The coupling of laser ablation/ICP-AES and ICP-MS spectrometry: A multiple applications tool. Can. J. Anal. Sci. Spec. 2005, 50 (5), 240-254.

125. Alloncle, G.; Gilon, N.; Lienemann, C. P.; Morin, S. A new method for quantitative analysis of metal content in heterogeneous catalysts: Laser ablation-ICP-AES. $C$. R. Chim. 2009, 12 (6-7), 637-646.

126. Weis, P.; Dücking, M.; Watzke, P.; Menges, S.; Becker, S. Establishing a match criterion in forensic comparison analysis of float glass using laser ablation inductively coupled plasma mass spectrometry. J. Anal. At. Spectrom. 2011.

127. Hickman, D. A.; Harbottle, G.; Sayre, E. V. The Selection of the best elemental variables for the classification of glass samples. Forensic Sci. Int. 1983, 23 (2-3), 189-212.

128. Jochum, K. P.; Weis, U.; Stoll, B.; Kuzmin, D.; Yang, Q.; Raczek, I.; Jacob, D. E.; Stracke, A.; Birbaum, K.; Frick, D. A.; Gunther, D.; Enzweiler, J. Determination of Reference Values for NIST SRM 610-617 Glasses Following ISO Guidelines. Geostand. and Geoanal. Res. 2011, 35 (4), 397-429.

129. Miller, J. N.; Miller, J. C. Statistics and Chemometrics for Analytical Chemistry; 5th ed.; Pearson Education Limited: England, 2005.

130. U.S. and World Cotton Outlook: June 2009. Economic Services, National Cotton Council. 2009.

131. Wakelyn, P.; Bertoniere, N. R.; French, A. D.; Thibodeaux, D. P.; Triplett, B. A.; Rouselle, M. A.; Goynes Jr., W. R.; Edwards, J. V.; Hunter, L.; McAlister, D. D.; Gamble, G. R. Cotton Fiber Chemistry and Technology; CRC Press: Florida, 2007. 
132. Dongre, A. B.; Bhandarkar, M.; Banerjee, S. Genetic diversity in tetraploid and diploid cotton using ISSR and microsatelite DNA. Indian Journal of Biotechnology 2007, 6, 349-353.

133. Wandersee, J. H.; Clary, R. M. When Cotton was King. Human Flower Project 2012.

134. Cotton: From seed to fiber here are the facts. Websites for Quilters 2012.

135. Wang, P.; Song, X.; Han, D.; Zhang, Y.; Liu, X. A study of root water uptake of crops indicated by hydrogen and oxygen stable isotopes: A case study in Shanxi Province, China. Agricultural Water Management 2010, 97, 475-482.

136. Kabata-Pendias, A. Trace Elements in Soils and Plants; third ed.; CRC Press: New York, 2001.

137. Gat, J. R. Comments on the stable isotope method in regional ground-water investigations. Water Resour. Res. 1971, 7, 980-993.

138. Watson, J. E.; Pepper, I. L.; Unger, M.; Fuller, W. H. Yields and leaf elemental composition of cotton grown on sludge ammended soil. J. Environ. Qual. 1985, $14,174-177$.

139. Noguerra, T. A. R.; Marques, M. O.; Muccucah, F. A.; Fonseca, I. M. Cotton Grown in Soil Treated with Limestone and Sewage Sludge Cadmium. J. Soil Sci. Nutr. 2007, 7, 74-87.

140. Brushwood, D. E.; Perkins Jr., H. H. Determining the Metal Content of Cotton. Anal. Tech. 1994, 26, 32-35.

141. Hewitt, E. J.; Smith, T. A. Plant Mineral Nutrition; Wiley and Sons: New York, 1975.

142. Moorby, J.; Besford, R. T. Volume 15B Inorganic Plant Nutrition; 1983.

143. Angelova, V.; Ivanova, R.; Delibatova, V.; Ivanov, K. Bioaccumulation and distribution of heavy metals in fibre crops (flax, cotton and hemp). Ind. Crops Prod. 2004, $19,197-205$.

144. Meier-Augenstein, W. Stable Isotope Forensics; John Wiley \& Sons, Ltd.: United Kingdom, 2010.

145. Zhao, H.; Guo, Y. W.; Zhang, B.; Sun, S.; Zhang, L.; Yan, J. Determining the Geographic Origin of Wheat Using Multielement Analysis and Multivariate Statistics. J. Agric. Food Chem. 2011, 59, 4397-4402.

146. Longobardi, F.; Casiello, G.; Sacco, D.; Tedone, L.; Sacco, A. Characterisation of the geographic origin of Italian potatoes, based on stable isotope and volatile compound analyses. Food Chem. 2011, 124, 1708-1813. 
147. Fernandez-Caceres, P.; Martin, M. J.; Pablos, F.; Gonzalez, A. G. Differentiation of Tea (Camillia sinesis) Varieties and Their Geographical Origin According to their Metal Content. J. Agric. Food Chem. 2001, 49, 4775-4779.

148. Rezic, I.; Steffan, I. ICP-OES determination of metals present in textile materials. Microchem. J. 2007, 85, 46-51.

149. Pranaityte, B. P. A.; Naujalis, E. Application of ICP-MS for the determination of trace metals in textiles. Chemija 2007, 18, 16-19.

150. Pszonick, L.; Hanna, A. N.; Suschny, O. Intercomparison of the determination of trace elements in cotton cellulose, IAEA/RL/97 Report; International Atomic Energy Agency: 83.

151. Gravel, J.-F. Y.; Boudreau, D. Study by focused shadowgraphy of the effect of laser irradiance on laser-induced plasma formation and ablation rate in various gases. Spectrochim. Acta, Part B 2009, 64, 56-66.

152. Brereton, R. G. Chemometrics for Pattern Recognition; John Wiley \& Sons, Ltd.: United Kingdon, 2009.

153. Johnson, R. A.; Wichern, D. W. Applied Multivariate Statistical Analysis; sixth ed; Pearson Education, Inc.: New Jersey, 2007.

154. Taylor, H. E. ICP-MS Practices and Techniques; Academic Press: New York, 2001.

155. Hoefs, J. Stable Isotope Geochemistry; forth ed.; Springer: Germany, 1997.

156. Hurley, J. M.; West, J. B.; Ehleringer, J. R. Stable isotope models to predict geographic origin and cultivation conditions. Sci. Justice 2010, 50, 86-93.

157. Galimov, E. M.; Sevastyanov, V. S.; Kulbachevskaya, E. V.; Golyavin, A. A. Isotope ratio mass spectrometry: $\delta^{13} \mathrm{C} \delta^{15} \mathrm{~N}$ analysis for tracing the origin of illicit drugs. Rapid Commun. Mass Spectrom. 2005, 19, 1213-1216.

158. Shibuya, E. K.; Sarkis, J. E. S.; Neto, O. N.; Mereira, M. Z.; Victoria, R. L. Sourcing Brazilian marijuana by applying IRMS analysis to seized samples. Forensic Sci. Int. 2006, 160, 35-43.

159. West, J. B.; Ehleringer, J. R.; Cerling, T. E. Geography and vintage predicted by a novel GIS model of wine delta O-18. J. Agric. Food Chem. 2007, 55, 7075-7083.

160. Taylor, E.; Carter, J. F.; Hill, J. C.; Morton, C.; Daeid, N. N.; Sleeman, R. Stable isotope ratio mass spectrometry and physical comparison for the forensic examination of grip-seal plastic bags. Forensic Sci. Int. 2008, 177, 214-220.

161. Daeid, N. N. M.-A. W.; Kemp, H. F. Investigating the provenance of un-dyed spun cotton fibre using multi-isotope profiles and chemometric analysis. Rapid Commun. Mass Spectrom. 2011, 25, 1812-1816. 
162. Craig, H. Isotopic variation in meteoric water. Science 1961, 133, 1702-1703.

163. Craig, H. Standard for reporting concentration of deuterium and oxygen-18 in natural waters. Science 1961, 133, 1833-1834.

164. Rozanski, K.; Araguas-Araguas, L.; Gonfiantini, R. Isotopic patterns in modern global precipitation. In: Climate change in continental isotopic records. Geophys. Monogr. 1993, 78, 1-36.

165. Isoscapes: Understanding movement, pattern, and process on Earth through isotope mapping; Springer: New York, 2010.

166. Gonfiantini, R.; Gratiziu, S.; Tongiorgi, S. Oxygen isotope composition of water in leaves. In Isotopes and radiation in soil-plant-nutrition studies, Intern. At. Energy Agency: Vienna, 1965; pp 405-410.

167. Dawson, T. E.; Ehleringer, J. R. Isotopic enrichment of water in the "woody" tissues in plants: implications for plant water source, water uptake, and other studies which use the stable isotopic composition of cellulose. Geochim. Cosmochim. Acta 1993, 57, 3487-3492.

168. Farquhar, G. D. B. M. M.; Henry, B. K. Interpretation of oxygen isotope composition of leaf material. In Stable Isotopes: integration of biological, ecological and geochemical processes, Griffiths, H., Ed.; BIOS Scientific Pub. Ltd.: 1998; pp 27-62.

169. Gan, K. S.; Wong, S. C.; Yong, J. W. H.; Farquhar, G. D. 180 Spatial Patterns of Vein Xylem Water, Leaf Water, and Dry Matter in Cotton Leaves. Plant Physiol. 2002, 130, 1008-1021.

170. Meier-Augenstein, W.; Chartrand, M. M. G.; Kemp, H. F.; St-Jean, G. An interlaboratory comparative study into sample preparation for both reproducible and repeatable forensic ${ }^{2} \mathrm{H}$ isotope analysis of human hair by continuous flow isotope ratio mass spectrometry. Rapid Commun. Mass Spectrom. 2011, 25, 3331-3338.

171. Nelson, S. T. A simple, practical methodology for routine VSMOW/SLAP normalization of water samples analyzed by continuous flow methods. Rapid Commun. Mass Spectrom. 2000, 14, 1044-1046.

172. Sharp, Z. D.; Atdorei, V.; Durakiewicz, T. A rapid method for determination of hydrogen and oxygen isotope ratios from water and hydrous minerals. Chem. Geol. 2001, $178,197-210$.

173. G.D.Farquhar; M.M.Barbour; J.S.Roden; G.D. Expressing leaf water and cellulose oxygen isotope ratios as enrichment above source water reveals evidence of a Peclet effect. Oecologia 2004, 138, 426-435.

174. Bowen, G. J. The Online Isotopes in Precipitation Calculator, version 2.2. Waterisotopes 2012. 
175. Station Information by State. Southeast Regional Climate Center 2012.

176. Average Statewide Temperature for Western U.S. States. National Climate Data Center 2012.

177. Weather Maps, Oregon Climate Services. Oregon Climate Change Research Institute 2012.

178. Field Crops Usual Planting and Harvesting Dates, October 2010;Agricultural Handbook No. $628 ; 10$.

179. American Heritage Science Dictionary; fourth ed.; Houghton Mifflin Company: New York, 2005.

180. Massart, D. L.; Vadeginste, B. G. M.; Deming, S. N.; Michotte, Y.; Kaufman, C. Chemometrics: A textbook; Elsevier: Amsterdam, 2003.

181. JMP Modeling and Multivariate Methods, version 10; SAS Publishing: 12.

182. Burdenski, T. Evaluating Univariate, Bivariate and Multivariate Normality Using Graphical and Statistical Procedures. Multiple Linear Regression Viewpoints 2000, 26, 15-28.

183. North American Free Trade Agreement (NAFTA). www. cbp. gov 2012.

184. What is CAFTA. www. caftaintelligence. com 2012.

185. Overview of Textiles: A Priority Trade Issue (PTI). www. cbp. gov 2011. 
VITA

\section{EMILY ROSE SCHENK}

Born, Cleveland, OH, USA

2007

Toxicology Intern

DRUGSCAN, Warminster, PA, USA

2007

B.Sc. in Forensic and Toxicological Chemistry, minor in Criminal Justice West Chester University of Pennsylvania, West Chester, PA

Pharmaceutical Intern

Cephalon Inc., Malvern, PA, USA

2008-2012

Teaching and Research Assistantship

Florida International University, Miami, FL, USA

2009

“Best Research Team” Award

North American Symposium on Laser-Induced Breakdown Spectroscopy (NASLIBS)

2010 Scholarly Forum: First Place Oral Presentation, Chemistry and Physics section

Florida International University, Miami, FL, USA

2011 Scholarly Forum: Second Place Oral Presentation, Chemistry and Physics section

Florida International University, Miami, FL, USA

2011

Doctoral Evidence Acquisition Fellowship

Florida International University, Miami, FL, USA

2011 American Society of Trace Evidence Examiners (ASTEE) Student Scholarship PUBLICATIONS

E.R. Schenk and J.R. Almirall. Elemental analysis of glass by laser ablation inductively coupled plasma optical emission spectrometry (LA-ICP-OES). Forensic Sci Int., 217, 222-8 (2012). 
E.R. Schenk and J.R. Almirall. Elemental analysis of cotton by laser-induced breakdown spectroscopy. Appl. Optics, 49, 153-160 (2010).

J.S. Falcone, Jr., J.L. Bass, M. Angelella, E.R. Schenk and K.A. Brensinger. The Determination of Soluble Silicate Composition Using ATR FT-IR. Ind. Eng. Chem Res., 49, 6287-6290 (2010). J.S. Falcone, Jr., J.L. Bass, P.H. Krumrine, K. Brensinger and E.R. Schenk. Characterizing the Infrared Bands of Aqueous Soluble Silicates. J. Phys. Chem, 114, 2438-2446 (2010).

E. Kordwitz and E. Schenk, JASCO Analytical SFC Start-up Manual, copyright JASCO 2008, all rights reserved by JASCO.

\section{PRESENTATIONS}

E.R. Schenk and J.R. Almirall. Oral presentation, Annual IFRI Forensic Science Symposium, March 9-10, 2012, Miami, FL.

E.R. Schenk and J.R. Almirall. Oral presentation, Intermediate Forensic Workshop, December 13-15, 2011, Miami, FL.

E.R. Schenk and J.R. Almirall. Oral presentation, Trace Evidence Symposium, August 8-11, 2011, Kansas City, MO.

E.R. Schenk. Oral presentation, Graduate Scholarly Forum, FIU, March 28, 2011, Miami, FL.

E.R. Schenk and J.R. Almirall. Poster presentation, PITTCON, March 14-17, 2011, Atlanta, GA. E.R. Schenk and J.R. Almirall. Poster presentation, The $6^{\text {th }}$ International Conference on LaserInduced Breakdown Spectroscopy, September 13-17, 2010, Memphis, TN.

E.R. Schenk. Oral presentation, Scholarly Forum, FIU, March 22, 2010, Miami, FL.

E.R. Schenk and J.R. Almirall. Oral presentation, AAFS 2010, February 25, 2010, Seattle, WA.

E.R. Schenk and J.R. Almirall. Poster presentation, NASLIBS, July 14, 2009, New Orleans, LA. E.R. Schenk and J.R. Almirall. Oral presentation, FAME, May 15, 2009, Orlando, FL.

E. Schenk, K. Brensinger and J. Ridinger. Oral presentation, NEAFS, November 3, 2007, Bolten Landing, NY. 